FHWA/IN/JTRP-2008/6

Final Report

ASSESSMENT OF AXIALLY-LOADED

PILE DYNAMIC DESIGN METHODS AND

REVIEW OF INDOT AXIALLY-LOADED

PILE DESIGN PROCEDURE

Dimitrios Loukidis

Rodrigo Salgado

Grace Abou-Jaoude

October 2008 


\section{TECHNICAL Summary}

INDOT Research

Technology Transfer and Project Implementation Information

TRB Subject Code: 62-1 Foundation Soils

Publication No. FHWA/IN/JTRP-2008/6, SPR-2856

October 2008

Final Report

\section{Assessment of Axially-Loaded Pile Dynamic Design Methods and Review of INDOT Axially-Loaded Design Procedure}

\section{Introduction}

The main goal of the present study is to make a comprehensive assessment of the existing methods for the dynamic analysis of pile driving, identify the shortcomings and propose improvements. A review of existing shaft and base soil reaction models used in dynamic pile analyses is done to evaluate their effectiveness and identify points that require improvement. Subsequently, we develop improved shaft and base reaction models for use in 1-D dynamic pile analysis. The proposed models are validated using experimental data recorded during driving of field piles and model piles. The procedures currently used by INDOT for the design of axially loaded piles are also examined. For this purpose, interviews were conducted with INDOT engineers and private geotechnical consultants involved in INDOT projects.

\section{Findings}

The interviews with INDOT engineers and consultants focused on the methods and procedures presently followed in deep foundation design projects. The methods and the computer software used by private consultants involved in INDOT projects for the design of axially loaded piles are consistent with those used by INDOT's geotechnical engineers. These methods and software follow FHWA guidelines and are in accordance with the standard practice in the U.S. Pile design is mostly based on Standard Penetration test (SPT) data and undrained shear strength measured in unconfined compression tests. Cone penetration tests are rarely performed, although both INDOT and private soil exploration companies have the necessary equipment. Pile drivability calculations and back-calculation of the pile capacity from dynamic test data are done using software that employs Smith-type soil reaction models. Pile driving monitoring and restrike tests are usually performed only in projects whose cost exceeds a certain limit. Static load tests are reserved mostly for research projects.

The methods for estimating the unit shaft and base resistances currently used by geotechnical engineers (INDOT or private consultants) have been developed over twenty years ago and have a large empirical content. There has been significant progress regarding methods for the calculation of unit base and shaft resistances. Numerous improved methods that are grounded on the physics and mechanics governing the development of pile resistance have been developed by combining experimental data with analysis. In the case of clayey soils, the differences between the state-of-the-practice methods and the updated methods are not large. In contrast, the formulation of the new methods for piles in sands has important fundamental differences with respect to traditional methods. The new methods for the base resistance in sands use as input either the relative density or the cone resistance directly. The recently developed methods recognize the fact that the limit base resistance is almost equal to the cone penetration resistance and that the ultimate unit base resistance in sands is smaller than the limit unit base resistance. Notably, the equations for $\beta$ adopted by FHWA guidelines yield substantially unconservative shaft resistance predictions.

The key components for accurate 1-D simulation of pile driving are the shaft and base soil reaction models. The reaction models currently used in practice are overly simple and 
rely on empirical constants. Significant effort has been made to develop improved soil reaction models that eliminate the empirical 'quake' parameter, have input parameters that are physically meaningful and attempt to adhere to the mechanics involved in pile driving.

Based on the shortcomings identified in the course of the thorough literature review process done as part of this project, we developed improved shaft and base reaction models that are consistent with the physics and mechanics of pile driving. The proposed shaft reaction model consists of a soil disk representing the near field soil surrounding the pile shaft, a plastic sliderviscous dashpot system representing the thin shear band forming at the soil-pile interface located at the inner boundary of the soil disk, and far-fieldconsistent boundaries placed at the outer boundary of the soil disk. The soil in the disk is assumed to follow a nonlinear stress-strain law.
The base reaction model consists of a nonlinear spring and a radiation dashpot connected in parallel. The initial spring stiffness and the radiation dashpot take into account the effect of the high base embedment. Radiation damping is applied only to the elastic component of the base displacement. Both shaft and base reaction models capture effectively soil nonlinearity, hysteretic damping, viscous damping and radiation damping. The input parameters are physically meaningful, thus reducing the level of empiricism to a minimum. The prediction using the proposed shaft and base reaction models compared favorably with measured pile driving data, unlike the predictions using Smith-type models. The pile driving simulations using the proposed models largely outperform those employing Smith-type models, especially in the case of hard driving conditions typically encountered as an endbearing pile approaches its final penetration depth.

\section{Implementation}

Since CPT-based methods are superior to methods that rely on SPT data, CPT should be performed more often in the field investigation of INDOT projects that involve deep foundations. CPT provides nearly direct estimate of the limit unit base resistance. Researchers have also found that the cone resistance correlates very well with unit shaft resistance. To take advantage of INDOT's CPT rig, CPTs could be performed more often for INDOT's deep foundation projects, and INDOT should continue to develop and refine CPT-based methods of design. Additionally, the new pile driving analysis methodology should be gradually used by INDOT in its deep foundation design projects.

The implementation of the research will enable INDOT to take advantage of the updated and improved unit resistance calculation methods contained in the present report in the design of deep foundations. These methods can then progressively replace the outdated methods that are currently used in INDOT projects. It will also allow use of a method of pile driving analysis that should allow both superior planning and superior quality control of INDOT's deep foundations work.

The best way to accomplish this goal would be to select deep foundation demonstration projects in which the research team would work together with INDOT during all the phases of planning, design and construction, assisting in implementation of the new design and analysis tools. This will allow further assessment of the accuracy and reliability of the equations proposed for the estimation of unit shaft and base resistances and improvement of methods using these design equations. Assessing the accuracy and the uncertainty of these methods is a prerequisite for the successful development of LRFD methodologies for axially loaded piles. The proposed implementation approach would allow development of LRFD methods in a way that would fulfill the ideals behind the LRFD philosophy. Workshops and seminars will also be organized in order to disseminate knowledge of the modern methods to consultants and INDOT engineers as well as show where the current methods fall short and can lead to potential cost overruns or other serious problems. INDOT has an opportunity to take leadership in the country in implementation of state-of-art knowledge and design technology in deep foundations projects.

We further suggest that the pile driving research done under this project should be continued and amplified. The solution scheme used in this project for the pile driving simulation can calculate the pile motion given unit soil resistance values along the shaft and at the base and the rated energy of the hammer. A more important practical application is the backcalculation of the pile base and shaft capacities based on the force and velocity time histories measured at the pile head during driving or during a re-strike test. Adding this capability to the present analysis framework requires the development of an efficient signal-matching algorithm. The developed pile driving analysis methodology can be of a value to both INDOT and geotechnical engineers and should be funded by JTRP. 


\section{Contacts}

For more information:

Prof. Rodrigo Salgado

Principal Investigator

School of Civil Engineering

Purdue University

West Lafayette IN 47907

Phone: (765) 494-5030

Fax: (765) 496-1364

E-mail: rodrigo@purdue.edu
Indiana Department of Transportation

Division of Research

1205 Montgomery Street

P.O. Box 2279

West Lafayette, IN 47906

Phone: (765) 463-1521

Fax: (765) 497-1665

\section{Purdue University}

Joint Transportation Research Program

School of Civil Engineering

West Lafayette, IN 47907-1284

Phone: (765) 494-9310

Fax: (765) 496-7996

E-mail: jtrp@ecn.purdue.edu

http://www.purdue.edu/jtrp 
Final Report

FHWA/IN/JTRP-2008/6

\title{
ASSESSMENT OF AXIALLY-LOADED PILE DYNAMIC DESIGN METHODS AND REVIEW OF INDOT AXIALLY-LOADED PILE DYNAMIC DESIGN METHODS
}

\author{
Dimitrios Loukidis \\ Post-Doctoral Research Associate \\ Rodrigo Salgado, P.E. \\ Professor \\ Grace Abou-Jaoude \\ Graduate Research Assistant \\ Geotechnical Engineering \\ School of Civil Engineering \\ Purdue University \\ Joint Transportation Research Program \\ Project No. C-36-36PP \\ File No. 6-14-42 \\ Prepared in Cooperation with the \\ Indiana Department of Transportation and \\ The U.S. Department of Transportation \\ Federal Highway Administration
}

The contents of this report reflect the views of the authors who are responsible for the facts and the accuracy of the data presented herein. The contents do not necessarily reflect the official views or policies of the Federal Highway Administration and the Indiana Department of Transportation. This report does not constitute a standard, specification or regulation.

Purdue University

West Lafayette, Indiana

October 2008 
TECHNICAL REPORT STANDARD TITLE PAGE

\begin{tabular}{|c|c|c|}
\hline $\begin{array}{l}\text { 1. Report No. } \\
\text { FHWA/IN/JTRP-2008/6 }\end{array}$ & 2. Government Accession No. & 3. Recipient's Catalog No. \\
\hline \multirow{2}{*}{\multicolumn{2}{|c|}{$\begin{array}{l}\text { 4. Title and Subtitle } \\
\text { Assessment of Axially-Loaded Pile Dynamic Design Methods and Review of } \\
\text { INDOT Axially-Loaded Pile Design Procedure }\end{array}$}} & $\begin{array}{l}\text { 5. } \quad \text { Report Date } \\
\text { October } 2008\end{array}$ \\
\hline & & 6. Performing Organization Code \\
\hline \multicolumn{2}{|l|}{$\begin{array}{l}\text { 9. Performing Organization Name and Address } \\
\text { Joint Transportation Research Program } \\
550 \text { Stadium Mall Drive } \\
\text { Purdue University } \\
\text { West Lafayette, IN 47907-2051 }\end{array}$} & 10. Work Unit No. \\
\hline \multicolumn{2}{|l|}{$\begin{array}{l}\text { 12. Sponsoring Agency Name and Address } \\
\text { Indiana Department of Transportation } \\
\text { State Office Building } \\
100 \text { North Senate Avenue } \\
\text { Indianapolis, IN } 46204\end{array}$} & 14. Sponsoring Agency Code \\
\hline \multicolumn{3}{|l|}{ 15. Supplementary Notes } \\
\hline \multicolumn{3}{|c|}{ Prepared in cooperation with the Indiana Department of Transportation and Federal Highway Administration. } \\
\hline
\end{tabular}

The general aim of the present research is to identify areas of improvement and propose changes in the current methodologies followed by INDOT for design of axially loaded piles, with special focus on the dynamic analysis of pile driving. Interviews with INDOT geotechnical engineers and private geotechnical consultants frequently involved in INDOT's deep foundation projects provided information on the methods and software currently employed. It was found that geotechnical engineers rely on static unit soil resistance equations that were developed over twenty years ago and that have a relatively large degree of empiricism. Updated and improved static design equations recently proposed in the literature have not yet been implemented in practice. Pile design relies predominantly on SPT data; cone penetration testing is performed only occasionally. Dynamic analysis of pile driving in standard practice is performed using Smith-type soil reaction models. A comprehensive review of existing soil reaction models for 1-dimensional dynamic pile analysis is presented. This review allowed an assessment of the validity of existing models and identification of their limitations. New shaft and base reaction models are developed that overcome shortcomings of existing models and that are consistent with the physics and mechanics of pile driving. The proposed shaft reaction model consists of a soil disk representing the near field soil surrounding the pile shaft, a plastic slider-viscous dashpot system representing the thin shear band forming at the soil-pile interface located at the inner boundary of the soil disk, and farfield-consistent boundaries placed at the outer boundary of the soil disk. The soil in the disk is assumed to follow a hyperbolic stress-strain law. The base reaction model consists of a nonlinear spring and a radiation dashpot connected in parallel. The nonlinear spring is formulated in a way that reproduces realistically the base load-settlement response under static conditions. The initial spring stiffness and the radiation dashpot take into account the effect of the high base embedment. Both shaft and base reaction models capture effectively soil nonlinearity, hysteretic damping, viscous damping, and radiation damping. The input parameters of the models consist of standard geotechnical parameters, thus reducing the level of empiricism in calculations to a minimum. Data collected during the driving of fullscale piles in the field and model piles in the laboratory are used for validating the proposed models.

\section{Key Words}

pile design, shaft resistance, base resistance, dynamic analysis, pile driving, numerical modeling, pile analysis.

\section{Distribution Statement}

No restrictions. This document is available to the public through the National Technical Information Service, Springfield, VA 22161
19. Security Classif. (of this report)

Unclassified

\author{
20. Security Classif. (of this page)
}

Unclassified

21. No. of Pages
188

188 


\section{TABLE OF CONTENTS}

Page

CHAPTER 1. INTRODUCTION ............................................................................. 1

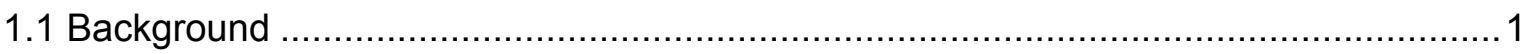

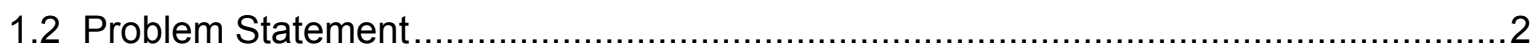

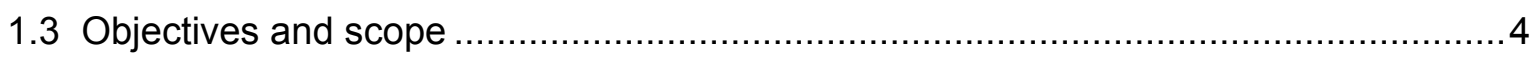

CHAPTER 2. REVIEW OF INDOT's DESIGN PROCEDURES .................................... 5

2.1 Foundation design process: tasks and responsibilities ........................................ 5

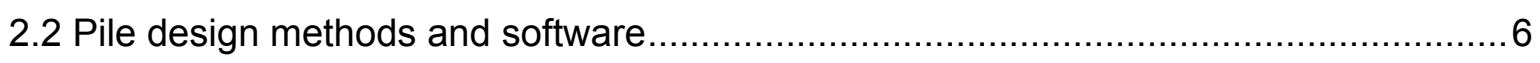

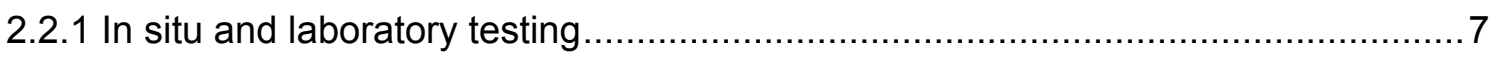

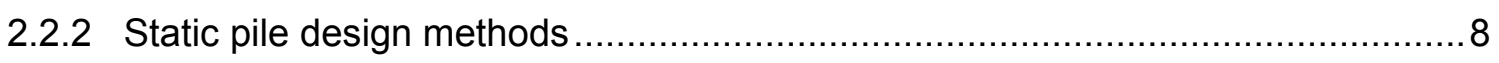

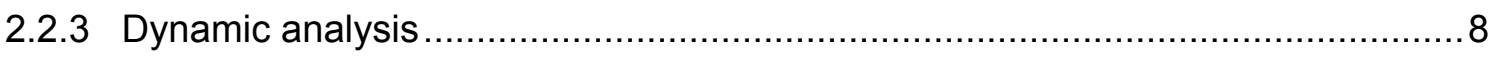

2.3 Advancements in pile design and their implementation .................................... 9

2.4. Conclusions and recommendations ........................................................... 10

CHAPTER 3. STATIC METHODS FOR THE DETERMINATION OF PILE CAPACITY ........13

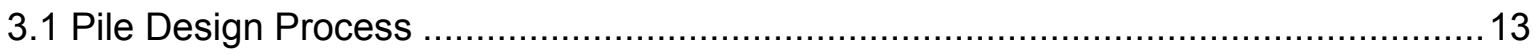

3.2 Property-based methods for unit base resistance in sand ................................. 17

3.2.1 Unit base resistance based on bearing capacity equation .............................. 17

3.2.2 Unit base resistance based on cone resistance estimate.................................. 18

3.3 Property-based methods for unit shaft resistance of nondisplacement piles in sand .. 21

3.3.1 Shaft resistance in sands according to Reese et al. (1976) ............................22

3.3.2 Shaft resistance in sands according to Stas and Kulhawy (1984) ......................22

3.3.3 Shaft resistance in sands according to O'Neill and Reese (1999)......................22

3.3.4 Shaft resistance in sands according to Loukidis and Salgado (2008) .................23

3.4 Property-based methods for unit shaft resistance of displacement and partial-

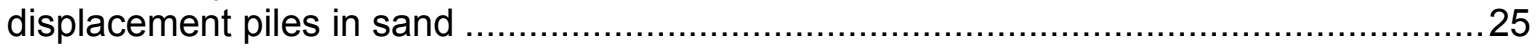

3.5 Property-based methods for unit base resistance of piles in clay .........................27

3.6 Property-based methods for unit shaft resistance of piles in clay ..........................28

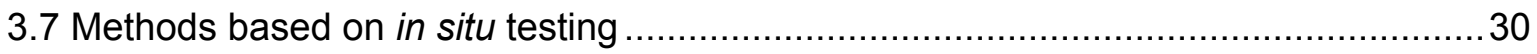

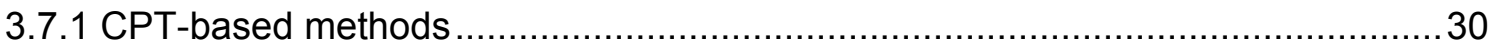

3.7.2 Limit shaft resistance method of displacement piles in sand by Jardine et al. (1998) 33

3.7.3 Limit shaft resistance method of displacement piles in sand by Randolph (2003)34 
3.7.4 Limit shaft resistance method of displacement piles in sand by Lehane et al. (2005) 34

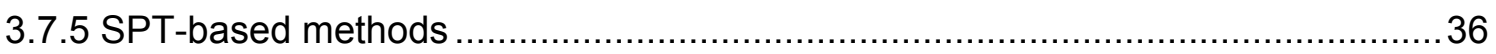

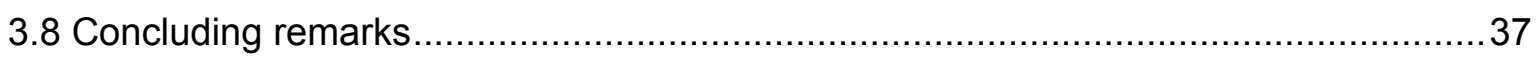

CHAPTER 4. ASSESSMENT OF DYNAMIC PILE ANALYSIS METHODS .......................39

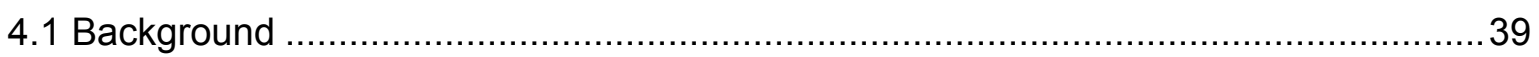

4.2 Mechanisms of soil reaction mobilization along pile shaft and base ........................42

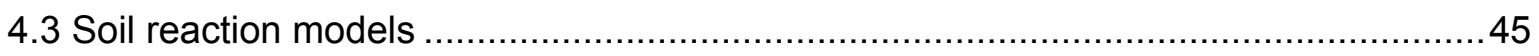

4.3.1 Smith (1960) shaft and base reaction models ............................................ 45

4.3.2 Base reaction model based on Lysmer's analog .........................................4 47

4.3.3 Shaft reaction model by Simons and Randolph (1985)/Randolph and Simons

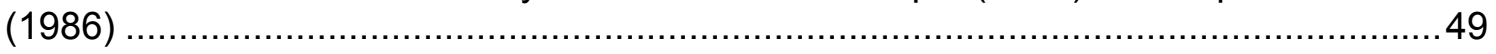

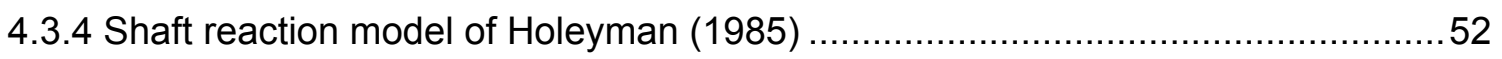

4.3.5 Shaft and base reaction models by Nguyen et al. (1988) ................................54

4.3.6 Base model by Deeks and Randolph (1995) ................................................55

4.3.7 Shaft model by El Naggar and Novak (1994) ..............................................57

4.3.8 Shaft model by Michaelides et al. (1998).................................................. 60

4.3.9 Continuum shaft and base models by Holeyman ......................................... 62

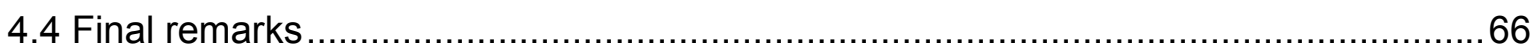

CHAPTER 5. PROPOSED SOIL REACTION MODEL FOR PILE DRIVING ANALYSIS .....69

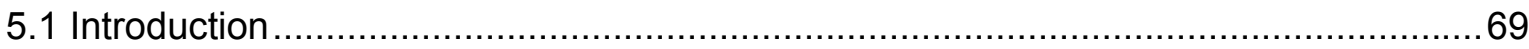

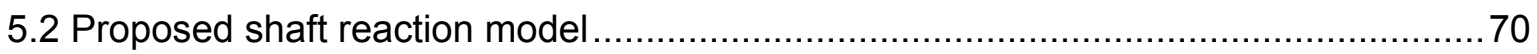

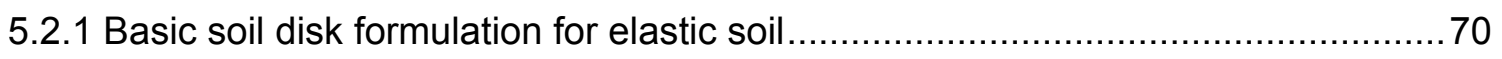

5.2.2 Free-field-consistent boundary conditions ............................................... 73

5.2 .3 Soil nonlinearity in soil disk formulation ................................................ 80

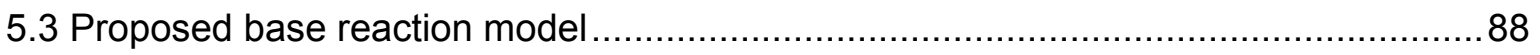

5.3.1. Effects of pile base embedment on the stiffness and radiation damping ............90

5.4 Soil reaction model input parameters ............................................................. 100

5.4.1 Selection of small-strain soil parameters ................................................ 101

5.4 .2 Selection of soil viscosity parameters ....................................................... 104

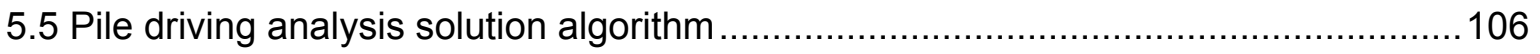

CHAPTER 6. FIELD AND MODEL PILE LOAD TESTS …........................................113

6.1 Field pile load test in Jasper County......................................................... 113

6.1.1 Laboratory and in situ testing ............................................................ 113

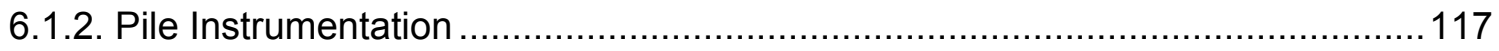


6.1.3 Pile driving and dynamic test results ................................................... 119

6.1.4 Static Pile Load Testing ........................................................................ 123

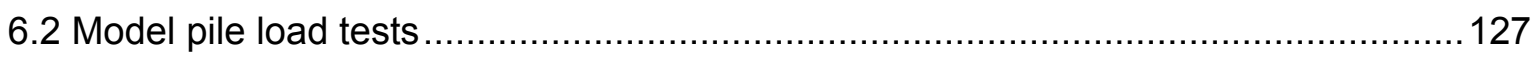

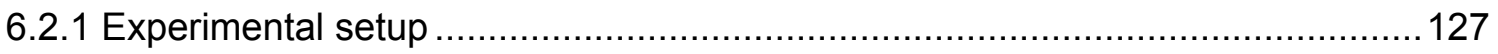

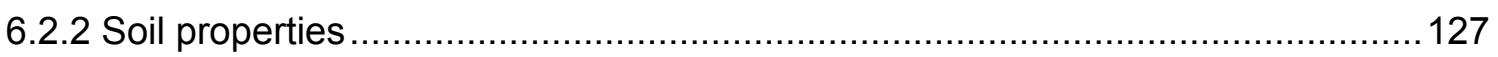

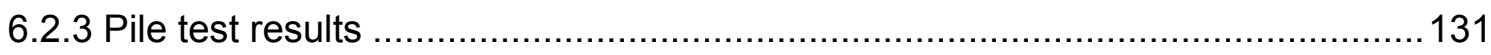

6.3. Field pile load test in Lagrange County ....................................................... 137

CHAPTER 7. NUMERICAL PREDICTIONS USING PROPOSED MODELS AND COMPARISON WITH PILE DRIVING DATA ........................................... 139

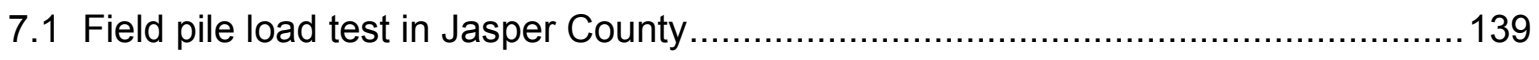

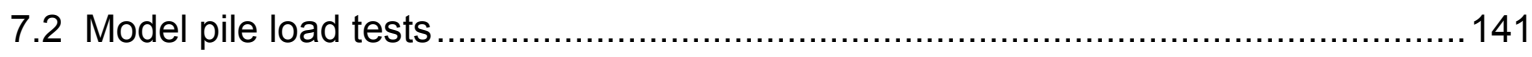

7.3 Field pile load test in Lagrange County ...................................................... 146

CHAPTER 8. CONCLUSIONS AND RECOMMENDATIONS ....................................149

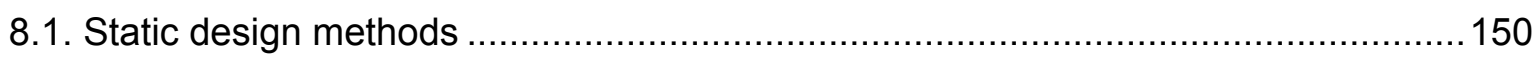

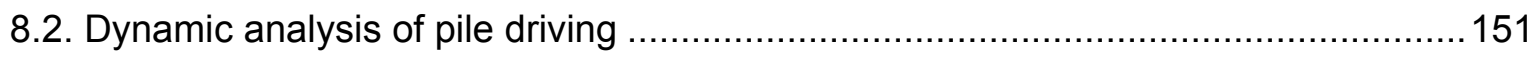

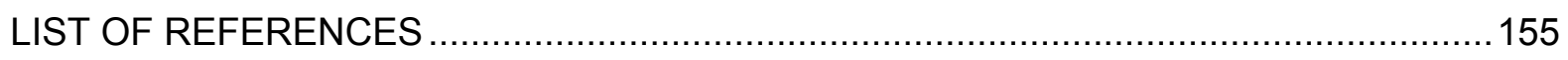

APPENDIX. FIELD AND MODEL PILE LOAD TEST PICTURES .............................. 167 


\section{LIST OF FIGURES}

Figure 3.1. Division into sublayers for the calculation of the limit shaft capacity................ 15

Figure 3.2. Mobilization of shaft and base resistances with pile settlement...................... 16

Figure 3.3. Comparison of ultimate base resistance values for displacement piles in sand from different soil-property-based methods.

Figure 3.4. Comparison of values of $\beta$ from different soil-property-based methods for sands.

Figure 3.5. Typical layering scheme based on $q_{c}$ values along the pile shaft and

recommended averaging of $\mathrm{q}_{\mathrm{c}}$ values in the bearing layer to find $q_{\mathrm{bL}}$.

Figure 3.6. Comparison of average $\beta$ data from tests on jacked piles at the Labenne test site (Lehane et al. 1993) with predictions from several methods. 36

Figure 4.1. Smith's numerical model for dynamic pile analysis: lumped mass discretization and soil reaction models

Figure 4.2. Energy transmission and absorption and deformation mechanism in the soil during pile driving: a) around the pile shaft, b) at the pile base. .........................4 44

Figure 4.3. Smith's reaction models: (a) for pile shaft and (b) for pile base.......................47

Figure 4.4. Base models following Lysmer's analogue: a) radiation dashpot always active and b) radiation dashpot ceases to act after onset of slippage. ........................48

Figure 4.5. Shaft reaction model according to Randolph and Simons (1986)....................50

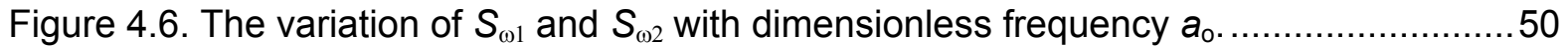

Figure 4.7. Shaft reaction model by Holeyman (1985).............................................52

Figure 4.8. Nguyen et al. (1988) reaction models: (a) for pile shaft and (b) for pile base....54

Figure 4.9. Base reaction model developed by Deeks and Randolph (1995).....................57

Figure 4.10. Shaft reaction model proposed by El Naggar and Novak (1994)....................59

Figure 4.11. Shaft reaction model proposed by Michaelides et al. $(1997,1998)$................61

Figure 4.12. Reaction models based on a continuum approach: a) base model by Holeyman (1988), b) shaft model by Holeyman et al. (1996). ....................................... 63

Figure 5.1. Soil disk around pile shaft and finite difference discretization.........................71

Figure 5.2. Use of radiation dashpot at the outer boundary of the continuum analysis domain.

Figure 5.3. The pring at the outer boundary of the analysis domain generates a displacement profile consistent with what is expected at the end of driving ..........75

Figure 5.4. Discretized near-field domain with spring and dashpot representing the far-field

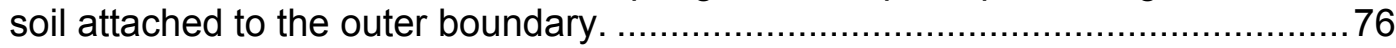

Figure 5.5. Soil response at different radial distances due to harmonic oscillation of the pile shaft. 
Figure 5.6. Soil response at different radial distances due to half sinusoidal pulse (no permanent displacement) at the pile shaft

Figure 5.7. Soil response at different radial distances due to quarter sinusoidal pulse with permanent displacement at the pile shaft.

Figure 5.8. Secant shear strain modulus as a function of shear strain: experimental curves by Vucetic and Dobry (1991) compared with predictions using eq. (5.11) and (5.12)

Figure 5.9. Stress-strain loops predicted by eq. (5.11) for two different values of the degradation parameter $b_{\mathrm{f}} \ldots$

Figure 5.10. Proposed shaft reaction model consisting of three parts: continuous near field, far-field consistent spring and radiation dashpot, and rheological shear band model at the soil-pile interface.

Figure 5.11. Proposed base reaction model consisting of a nonlinear spring and a radiation dashpot.

Figure 5.12. Mesh and boundary conditions used in dynamic FLAC analyses of circular footing at the bottom of cylindrical well.

Figure 5.13. Variation of depth factor $D_{f}$ for static footing stiffness with embedment ratio D/B.

Figure 5.14. Velocity field in dynamic FLAC analysis showing wave propagation in all directions.

Figure 5.15. Formulation of 1-D dynamic analysis: discretization of pile into lumped masses with soil reactions applied to each lumped mass.

Figure 6.1. Plan view of field pile test layout ....................................................... 114

Figure 6.2. Cone penetration test results ......................................................... 116

Figure 6.3. Comparison between CPT and SPT test results ...................................116

Figure 6.4. Location of strain gages on pipe pile .................................................. 118

Figure 6.5. Location of accelerometers $A 1$ and $A 2$ and strain transducers F1 and F2 at pile

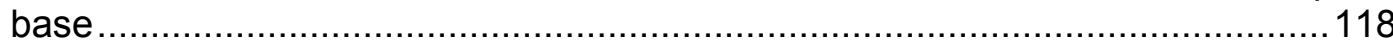

Figure 6.6. Driving resistance of closed-ended pipe pile ........................................119

Figure 6.7. Velocity records at pile top for different hammer blows (pile MP1, Jasper County).

Figure 6.8. Displacement records at pile top during different driving stages of pile MP1 in Jasper County

Figure 6.9. Displacement time histories at pile base for different hammer blows (pile MP1, Jasper County). 122

Figure 6.10. Force records at pile base during final driving stages (pile MP1, Jasper County).

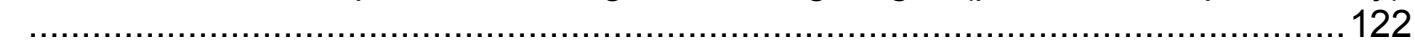

Figure 6.11. Reaction frame system and setup for static load test 123

Figure 6.12. Load-settlement curves for field static load tests on pile MP1 in Jasper County 
Figure 6.13. Load transfer curves from the static load tests on pile MP1 in Jasper County.

Figure 6.14. Unit shaft resistance versus depth.................................................. 126

Figure 6.15. Model pile and drop hammer assembly .......................................... 128

Figure 6.16. Arrangement of strain gages in model pile II ........................................129

Figure 6.17. Driving resistance in Tests No.1 and No.2 ................................... 131

Figure 6.18. Driving resistance in Tests No.1 and No.2 ........................................ 132

Figure 6.19. Displacement and velocity records from Test No.1 ................................. 132

Figure 6.20. Displacement and velocity records from Test No.2 .................................. 133

Figure 6.21. Displacement and velocity records from Test No.3 .................................. 134

Figure 6.22. Displacement and velocity records from Test No.6 ................................135

Figure 6.23. Load-settlement curves for static load tests No. 1 and No. 2 ....................136

Figure 6.24. Load-settlement curves for static load tests No. 4 and No. 5 ....................136

Figure 6.25. SPT and CPT data for test site at Lagrange County................................138

Figure 6.26. Distribution of limit unit shaft resistance along pipe pile at Lagrange County. 138

Figure 7.1. Predicted and measured pile head displacement time history for closed-ended steel pipe pile in Jasper County. 140

Figure 7.2. Predicted and measured pile head displacement time history for model pile test No.1: medium Ottawa sand with $D_{R}=20 \%$.

Figure 7.3. Predicted and measured pile head displacement time history for model pile test No.2: medium Ottawa sand with $D_{\mathrm{R}}=95 \%$.

Figure 7.4. Predicted and measured pile head displacement time history for model pile test No.3: fine Ottawa sand with $D_{\mathrm{R}}=61 \%$.

Figure 7.5. Predicted and measured pile head displacement time history for model pile test No.4: fine Ottawa sand with $D_{R}=36 \%$.

Figure 7.6. Predicted and measured pile head displacement time history for model pile test No.5: fine Ottawa sand with $D_{\mathrm{R}}=80 \%$.

Figure 7.7. Predicted and measured pile head displacement time history for model pile test No.6: fine Ottawa sand with $D_{R}=80 \%$.

Figure 7.8. Predicted and measured pile head displacement time history for closed-ended steel pipe pile in Lagrange County. 147

Figure A.1 Welding of a vibrating wire strain gage to the pile shaft ................................ 167

Figure A.2 Spot-welded gage on smoothed pile surface ..........................................167

Figure A.3 Gage protected with steel cover and silicon caulking ..................................168

Figure A.4 ICE-42S diesel hammer used in pile driving ........................................... 168

Figure A.5 Pile marked every $1 \mathrm{ft}$ for blow count records ..........................................169

Figure A.6 Static pile load test setup.............................................................. 169 
Figure A.7 Electric resistance strain gage attached to the pile base load cell ..................170

Figure A.8 Strain gages and accelerometers attached to the pile top .......................... 170

Figure A.9 Test pit and model pile setup.............................................................. 171

Figure A.10 Close-up on drop hammer and model pile top .......................................171

Figure A.11 Load cell and hydraulic jack setup for model pile ..................................... 172

Figure A.12 Reaction beam and counter weights for model pile test..............................172 


\section{LIST OF TABLES}

Table 3.1. Summary of recommended $c_{b}$ values for calculating unit base resistance of different pile types (Salgado, 2008)

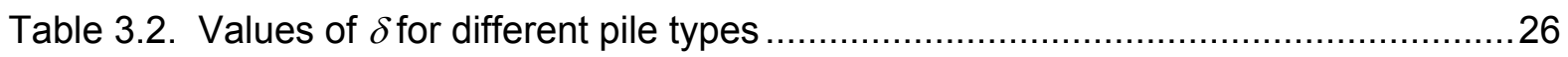

Table 3.3. Summary of recommended design equations for calculating unit base resistance of different pile types (Salgado, 2008)

Table 3.4. Summary of recommended $\alpha$ values for calculating limit shaft resistance of different pile types (Salgado, 2008).

Table 3.5. Summary of $c_{\mathrm{s}}$ values for calculating limit shaft resistance of different pile types 32

Table 3.3.6. Summary of $c_{b}$ values for calculating ultimate base resistance of different pile types

Table 3.7. Summary of $c_{s}$ values for calculating limit shaft resistance of different pile types 33

Table 4.1. Summary of shaft reaction model

Table 4.2. Summary of base reaction model 68

Table 5.1. Summary of results from FLAC simulations of embedded vibrating footing. 95

Table 5.2. Exponent $\mathrm{k}$ for eqs. (5.42) and (5.43) 103

Table 5.3. Estimation of $K_{2, \max }($ Seed and Idriss 1970) 103

Table 5.4. Values of $K_{\mathrm{u}}$ after Weiler (1988).... 104

Table 5.5. Correlations between $G_{\max }$ and cone resistance $q_{\mathrm{c}}$ (from Kramer 1996)..... 104

Table 6.1. Soil properties for samples obtained from Jasper County pile load test site......115

Table 6.2. List of model pile tests. 129

Table 7.1. Experimental data and numerical predication on pile set for model piles tests. 146 


\section{CHAPTER 1. INTRODUCTION}

\subsection{Background}

Pile foundations are often used to transfer large superstructure loads to the ground at sites where shallow foundations cannot be used due to the presence of soft clay or loose sand layers. Pile foundations are also capable of resisting large tensile or lateral loads. Pile reaction to compressive axial loads comes from two sources: shaft resistance and base resistance. The mobilization of limit shaft resistance requires small pile head displacements, whereas much larger head displacements are required for full mobilization of base resistance. Depending on the installation method, most piles fall into one of two main categories: nondisplacement piles or displacement piles. Nondisplacement piles are cast-inplace piles installed by first excavating the soil and then pouring concrete or grout in the created space. This installation process causes only small disturbance to the surrounding soil, largely preserving the initial stress state and soil density. Nondisplacement piles are practically synonymous to drilled shafts (bored piles). Displacement piles are inserted into the ground by driving or jacking without prior removal of the soil from the ground. Displacement piles are made out of steel (e.g. steel pipe piles, H-piles), concrete (prestressed or not), or wood. From the pile design perspective, there are large differences in the response to axial loading between nondisplacement and displacement piles. Generally, displacement piles have larger capacity than nondisplacement piles. In addition, pile settlement required for full mobilization of the base resistance is smaller in displacement piles than in nondisplacement piles.

The methods used for the determination of pile capacity prior to installation belong to two main categories: 1) methods based on soil properties and 2) methods based on in situ tests such as SPT and CPT. These design methods constitute the so-called static methods. Although pile foundations are very common today and are used often for supporting heavy and important structures, the most popular and well established methods for the determination of the shaft and base resistances in practice still contain a significant degree of uncertainty. The majority of the existing methods contain also a large degree of empiricism, which limits their effectiveness and the broadness of their applicability. As a consequence, foundation engineers often rely on dynamic or static pile testing for verifying 
the pile capacity and reassessing the foundation design prior to or at intermediate stages of construction. Pile load testing in the field adds a significant amount to the overall cost of the project. The most common pile tests are static load tests, pile monitoring during driving (for driven piles), and re-strike tests. Although simpler in concept, static load tests are very costly and are reserved only for large-budget and important projects. The less expensive alternative, suitable for more routine projects, is the use of dynamic pile tests, either in the form of pile driving monitoring or in the form of re-strike tests. Pile monitoring consists of recording the pile acceleration and axial strain at the pile head during driving. The pile head velocity and displacement histories can be extracted from the recorded acceleration history through numerical integration. The recorded strain is used for calculating the axial force history at the pile head. This information can be used to deduce the static pile capacity. In pile re-strike tests, the pile head is struck by a hammer after it has been fully driven to the ground. A similar type of test can also be performed on drilled shafts (e.g., Newton's APPLE $^{\mathrm{TM}}$ load testing by GRL). Determination of the pile capacity from static pile tests is simple, direct and straightforward. Pile capacity estimation from dynamic tests has always been more challenging, requiring sophisticated dynamic analysis.

\subsection{Problem Statement}

The difficulties of the commonly used static design methods in accurately predicting the pile capacity often require the use of field pile load tests, resulting in a significant increase of the project cost. Due to practical and economic reasons, dynamic pile testing is performed much more frequently than static pile load tests. Early efforts in interpreting dynamic pile test data relied on empirical formulas. These formulas are based on a simple energy concept: the net energy transmitted to the pile head should be equal to the work done by the total pile resistance on the measured vertical pile displacement (pile set). Such formulas were adopted by various design codes throughout the United States and abroad. The most wellknown among these formulas are the Engineering News Record (ENR) formula and Gates' formula. Researchers noticed early on the limitations of the empirical formulas. It became obvious that proper analysis requires pile driving to be treated as a wave transmission problem and analyzed considering the wave equation. Significant effort has been put in finding different ways to analyze the problem by taking into account the actual physics and mechanics of the pile driving problem and the effects of pile-soil interaction. 
The major advancement in pile dynamics was the consideration of the one-dimensional dynamic analysis (Smith 1960). In this approach, the pile is modeled as a series of lumped masses interconnected by springs and the problem is solved using numerical integration. In the course of the following decades, several improvements were made to the original work by Smith (e.g. Lowery et al. 1969; Hirsch et al. 1976; Goble and Rausche 1976, 1986; Simons and Randolph 1985; Lee et al. 1988; Rausche et al. 1994; Hussein et al. 1995). Alternative techniques were also proposed for analyzing pile driving, such as finite element analysis (e.g. Coutinho et al. 1988; Borja 1988; Nath 1990; Deeks 1992; Mabsout and Tassoulas 1994; Mabsout et al. 1995; Liyanapathirana et al. 2000; Masouleh and Fakharian 2007) or closed-form solutions (e.g. Glanville et al. 1938; Hejazi 1963; Parola 1970; Wang 1988; Zhou and Liang 1996; Warrington 1997).

Goble et al. developed a method based on the wave equation that allows the calculation of pile capacity using force and velocity measurements at the pile head. A number of simple closed-form solutions were developed based on this concept: the Case Method (Goble et al. 1975, 1980), the Impedance Method (Beringen et al. 1980) and the TNO Method (Foeken et al. 1996). Following similar lines, Rausche (1970) proposed the signal matching technique and developed the computer program CAPWAP (Rausche et al. 1985). CAPWAP performs 1-dimensional dynamic analysis (Smith's lumped mass approach) aiming to obtain good match between the measured and predicted force and velocity time histories at the pile head by changing the static soil resistance values along the pile shaft and at the pile tip. CAPWAP distinguishes between the static and dynamic resistances and thus allows the prediction of the static bearing capacity from the dynamic measurements. Currently, CAPWAP constitutes the state-of-practice method/software for the interpretation of dynamic pile load tests. The program GRLWEAP, developed by the same researchers, employs similar numerical methodology and is used before pile installation for determining pile drivability and producing bearing graphs.

Although CAPWAP is considered more reliable than other dynamic methods, it utilizes soil reaction models that are simplistic and empirical (Randolph 2003). Kim et al. 2002, Lee et al. 2003, and Paik et al. 2003 found that it underpredicts significantly the load capacity of both closed- and open-ended piles for the conditions investigated by these authors. Regarding drivability, INDOT engineers have reported that predictions of the final driving depths and, thus, of the pile capacity made by design engineers are often unsatisfactory. These difficulties may potentially arise from the inadequate soil reaction modeling. Given the large cost associated with dynamic testing and its importance in the 
final foundation design of important projects, dynamic pile analysis needs to be as reliable as possible. The knowledge of dynamic soil behavior and of the mobilization of soil resistance around the pile has advanced significantly, allowing the development of more rigorous and realistic soil reaction models that will help in improving the reliability of dynamic pile analysis.

\subsection{Objectives and scope}

The present research effort aims to identify shortcomings in the current axially-loaded pile design procedure used in INDOT's projects and examine the possibility of implementing new advances and knowledge in dynamic pile analysis. To achieve this goal, the present research project consists of the following tasks:

(1) Review of the axially-loaded pile design process followed by INDOT and identification of the main problems and areas of improvement that would lead to more economical and safer projects in the future.

(2) Comprehensive assessment of the static design methods currently available for the design of axially loaded piles and identification of the most accurate methods and procedures.

(3) Evaluation of current dynamic pile analysis methods.

(4) Development improved dynamic analysis method based on soil reaction models that obey the true physics and mechanics of pile driving. 


\section{CHAPTER 2. REVIEW OF INDOT's DESIGN PROCEDURES}

A series of interviews were conducted by Geoffrey Henggeler during the summer of 2004. The interviewees were two INDOT engineers and two private geotechnical consultants involved in INDOT projects. The focus of the interviews was to develop an overview of the procedures and practices followed in the design of axially loaded piles. The information collected focuses on the decision process in selecting foundation type, the methods/software used in pile design, and the data collection and exchange during and after the construction phase. The following sections present a compilation of the information collected during the interviews.

\subsection{Foundation design process: tasks and responsibilities}

There are four different parties that are involved in the foundation design and construction. These are INDOT, the project's design consultant (Architect/Engineer or $A / E$ ), the geotechnical consultant, and the contractor. INDOT is the state agency responsible for providing and maintaining public roads for transportation within the state, and thus acts as the project owner. It determines the project specifications and requirements and controls the bid process. The design consultant produces the design of the superstructure.

The geotechnical consultant is responsible for the field investigation, the in situ and laboratory testing, the foundations design, and the production of the geotechnical report. INDOT can act also as design consultant and/or as geotechnical consultant. The INDOT geotechnical department undertakes the geotechnical consulting for the majority of the projects, in which case INDOT geotechnical engineers prepare and submit the geotechnical engineering report directly to the project designer (A/E). INDOT hires private geotechnical consultants when the INDOT geotechnical lab is too busy to perform additional work. Geotechnical consulting is also outsourced to private consultants when a project is being performed under Fast-Track procedure and the INDOT geotechnical department is not able to keep up with the rapid pace of the project schedule. Fast-Track is an accelerated design and construction schedule for important projects. INDOT selects the private geotechnical consultant from a list of preapproved companies. 
The geotechnical engineer (INDOT or private) receives preliminary design information from the design consultant. The design consultant provides basic design information to be used as input for the production of the geotechnical report and the foundation design, such as bridge type, location of bents or piers, superstructure loading to the foundation system, and whether a project utilizes open bents or a pile cap. A typical geotechnical report includes field investigation and laboratory test results, the inferred subsurface profile, design calculations, pile foundation design (pile type, number of piles and layout, pile cross-section, and installation depth), driving recommendations, and sometimes the recommended pile spacing. Once the geotechnical consultant completes the geotechnical engineering report, it is subsequently reviewed by INDOT's senior geotechnical engineers who may occasionally suggest amendments. Private consultants may contact INDOT before completion of the geotechnical report if problems arise during foundation design. The foundation elements in the approved geotechnical engineering report are considered complete and are implemented in the project's design by the Architect/Engineer without additional changes. The geotechnical report does not include final drawings. The design consultant $(A / E)$ finalizes the design of the deep foundation system based on the contents of the geotechnical engineering report and incorporates deep foundation plans into the final engineering drawings.

Contractors are not allowed to propose design alternatives unless they present a Cost Reduction Incentive (CRI) proposal. The proposed CRI must include a complete analysis, additional testing results, and cost saving documentation to a sufficient level of detail. The CRI alternatives need to be approved by the designer (A/E). The CRI proposal is reviewed by INDOT engineers and often by the geotechnical consultant. INDOT geotechnical engineers must always be consulted in case modifications need to be made to the foundation system during construction.

\subsection{Pile design methods and software}

The vast majority (around $90 \%$ ) of INDOT bridge projects utilize deep foundation elements. Pile foundations are also used in certain retaining wall projects. Driven piles are used much more frequently (roughly $80 \%$ of the time) than drilled shafts; this is mainly due to local contractor capabilities. Drilled shafts are usually considered, at the request of the design consultant, when it is desired to have a single pile or column load supported by a single 
foundation element (pile). Drilled shafts are particularly suitable for such cases because they are available in much larger cross-sectional sizes than typical driven piles, thus being able to provide the large design capacities that are required.

The practice followed by private consultants in terms of design methods, software and factor of safety values is consistent with that followed by INDOT geotechnical engineers. The pile design has been based on the Working Stress Design (WSD) approach but that is changing new with the new LRFD requirements. In the absence of pile load testing, the value of the factor of safety is equal to 2.5 . The factor of safety is applied to the total axial load capacity (i.e., no separate factors of safety are considered for shaft resistance and tip resistance). The safety factor may be reduced to 2.0 in projects involving pile load tests. In general, INDOT will not use a safety factor less than 2.

Decision on the final foundation system configuration is controlled by two main factors: cost and constructability. Additional considerations include structure type, prior experience, local practice, and capabilities of local contractors. The later is an important consideration, as contractors able of installing certain types of foundation systems may not be available in all parts of Indiana. Hiring a pile installation sub-contractor from elsewhere may significantly increase the project cost. Optimization of the pile design (pile type and geometry) is accomplished by the geotechnical consultant (private firm or INDOT) during the preparation of the geotechnical engineering report. The pile group arrangement or orientation is rarely considered as a variable during the optimization process.

\subsubsection{In situ and laboratory testing}

In the vast majority of projects, the only in situ test performed is the Standard Penetration Test (SPT). SPT testing is an integral part of the soil borings performed in the context of the field investigation. Shelby tube samples are taken in the case of cohesive soils. Unconfined compression tests (UC) or UU tests are performed on the collected specimens for determining the undrained shear strength of the cohesive soil. INDOT engineers usually rely on the SPT blow counts and UC (or UU) test data for determining the pile capacity in sandy soils and clayey soils, respectively. More advanced laboratory tests are available in INDOT laboratories or private geotechnical laboratories, but these tests are rarely used for projects involving deep foundation design. The private geotechnical consultants interviewed, as well as INDOT's geotechnical office, have the capability of performing Cone Penetration Test (CPT). However, CPT is used infrequently because of the perceived difficulties in performing 
this test in coarse-grained soils present in Indiana. Reaching large depth has also been stated as one of the limitations of the CPT. CPT testing is being used by INDOT more frequently than in the past, but it still represents only a small fraction of the in situ tests performed. Pressuremeter testing and dilatometer testing are rarely performed and, when performed exclusively by private firms. The in situ and laboratory tests undertaken by the INDOT geotechnical division are performed and monitored by technicians. Geotechnical engineers are present during testing only in projects involving complex issues, such as soils with large amounts of organic material, or when conducting complicated testing.

\subsubsection{Static pile design methods}

Both INDOT and private consultants calculate the capacity of axially loaded piles using specialized software. In the absence of subsequent pile load testing, the entire foundation system design relies on the output of these computer programs. The programs S-PILE and DRIVEN are used for the design of driven piles. The development of these two programs was sponsored by FHWA. Currently, INDOT is phasing out the use of S-PILE in favor of DRIVEN, although both programs are still used. The program SHAFT (Ensoft, Inc.) is used for the design of drilled shafts. The interviewees are generally satisfied with the performance of these programs. Moreover, the geotechnical consultants feel very familiar with the programs and appreciate their user-friendliness (setting aside S-PILE, all of them are Windows based). Pile setup is considered only if conditions warrant it.

\subsubsection{Dynamic analysis}

The programs WEAP and GRLWEAP are used for dynamic pile analysis to assess the drivability of a given pile type (when drivability is of concern). These programs have been praised for their excellent support and training provided by the software developer. The bearing graph made available to the contractor, the supervising engineer and field personnel during construction is usually produced using these programs. Alternatively, a formulabased method may be used for producing the bearing graph. The bearing graph is necessary for directing and monitoring the pile driving process. A bearing graph allows assessment of pile drivability and estimation of pile capacity with depth in the field. The pile driving records are collected by the project engineer and transmitted to INDOT. These are 
used for assessing the effectiveness of the driving system rather than evaluating the quality of construction.

INDOT considers performing pile load tests when the total foundation cost exceeds $\$ 100,000$ or in projects involving a large number of piles, large pile loads, and difficult driving conditions. The availability of pile load test data allows the use of lower factor of safety, which may result in a reduction of the foundation system cost. The vast majority of pile load testing consists of dynamic tests (PDA); static pile load tests are mostly performed in research projects. The responsibility of PDA testing lies exclusively to INDOT. In the past, INDOT used to contract specialized consulting firms to perform PDA tests. INDOT acquired the PDA equipment approximately five years ago and trained its geotechnical engineers on the use of the equipment. Pile integrity and crosshole testing for verifying the quality of installed drilled shafts are rarely performed.

It has been pointed out that there is little interaction between INDOT and the private geotechnical consultants who design the foundation system. The results of the PDA are not directly communicated to the private geotechnical consultant by INDOT; PDA data is occasionally received from the design consultant instead. After submitting a geotechnical engineering report to INDOT or to the design consultant, private geotechnical consultants receive only limited feedback.

\subsection{Advancements in pile design and their implementation}

Private geotechnical consultants acquire knowledge about advancements in pile engineering from journals, publications, conferences, seminars, short courses, and interaction with other firms. Private consultants also try maintaining their own databases and developing their own pile capacity correlations applicable to soil conditions encountered in the state of Indiana. Continuing education activities, such as seminars, lectures, and short courses, are usually hosted and/or sponsored by academic research institutions, the FHWA, and the pile foundation industry. The Indiana ASCE Geotechnical Committee meets several times per year to share information related to the design and construction of deep foundations. INDOT is actively engaged in advancing the state of knowledge and practice in the field of geotechnical engineering through research programs run in collaboration with academic institutions and FHWA. 
The interviewees indicated a number of aspects of pile engineering in which advancements are needed. It is recognized that future research needs to shed light on the physics and mechanics involved in pile driving and in pile setup. Studying the pile-soil interaction in pile groups and its effect to the capacity of axially-loaded piles would also be beneficial. Improvements in pile design could be made by developing more reliable correlations between in situ test data and pile resistances. It will be of value to develop a unified pile analysis and design process based on a well defined set of suitable and updated set of design equations. INDOT geotechnical engineers indicate that, despite certain comfort and familiarization with the current state-of-practice, continuous improvement of the pile design practice is important and very beneficial. It is estimated that INDOT implements new advancements in deep foundation engineering every year or two. It has been pointed out that dissemination of software sprung from recent research efforts is practically possible only if the software is made to be Windows-based.

Particular mention was made regarding the limitations of the Fast-Track procedure. The primary limitation is that the parties involved in foundation design do not have the time to interact with each other. Interaction between the geotechnical consultant and design consultant is necessary for producing good engineering designs. Good interaction between these two parties will greatly improve the quality of the final design and minimize occurrence of problems arising during construction and the service life of the project.

\subsection{Conclusions and recommendations}

The main points extracted from the content of the interviews can be summarized as follows:

1) The design procedures followed by INDOT's geotechnical engineers and private geotechnical consultants are consistent (e.g., methods, software).

2) Most of the time, the design recommended in the geotechnical report is not modified by the contractor. A cost reduction incentive $(\mathrm{CRI})$ program allows contractors to propose modifications.

3) Pile design is mostly based on SPT data and $s_{u}$ determined from unconfined compression tests. CPT is performed only occasionally.

4) The computer programs DRIVEN and S-PILE are used for the design of driven piles. The program SHAFT is used in the case of nondisplacement piles (drilled shafts). 
5) Pile design has been based on the Working Stress Design method. A factor of safety equal to 2.5 is used, unless pile load tests are performed, in which case the factor of safety may be reduced to 2.0 .

6) The program WEAP (or GRLWEAP) is used for analyzing pile drivability. The bearing charts used during construction are generated using GRLWEAP or empirical formulas.

7) PDA tests may be performed depending on the cost of the project, the pile capacity, and the driving conditions. Static load tests are rarely performed.

It can be concluded that deep foundation design procedures followed by INDOT and private firms are in accordance with standard and acceptable practices followed in the United States and abroad. There are though certain aspects of the design procedures and practices in which improvements can be made. Based on the information collected from the interviews and the experience gathered from previous JTRP projects, we can make the following comments and recommendations:

a) The pile design software used for the static calculations follows the methods adopted in FHWA reports and guidelines and implemented in pile design software (DRIVEN, S-PILE, SHAFT). These are strongly empirical property-based methods developed in the 1960's, 70's and 80's. There is the need to implement in pile analysis software the recent advancements regarding unit resistance calculation. These more modern methods are also often based on the physics and mechanics involved in the mobilization of the pile capacity.

b) The soil reaction models used currently in the dynamic analysis of piles are overly simple and rely on empirical constants. There is the need to develop and implement soil reaction models that are consistent with the mechanics involved in pile driving and have input parameters that are physically meaningful. Advancement in this field will improve the reliability of pile driving analysis.

c) There is a worldwide trend of shifting geotechnical design from WSD towards LRFD. Establishing and promoting Load and Resistance Factor Design (LRFD) into pile engineering will lead to more reliable designs. The adoption of modern unit resistance equations is a prerequisite for the successful development of LRFD methodologies for piles.

d) The CPT should be performed more frequently in projects involving deep foundations. CPT-based methodologies are more reliable than SPT-based ones. In 
the case of pile design, CPT is the method of choice because the mechanics involved in the problem of pile installation and subsequent axial loading are very similar to those in cone penetration. CPT is used with great success abroad in coarse grained soils and at depths typically reached by pile tips. If a boulder or cobble is encountered during penetration, relocation of the rig just a few feet away often solves the problem.

e) When determining the undrained shear strength of clay samples in the laboratory, UU tests should be preferred over UC tests, especially in the case of soft clays.

f) INDOT usually designs piles to have the required capacity by varying the pile length. It is recommended to design the pile by varying the pile cross-section and targeting pile termination in the same bearing layer whenever possible. This practice helps in limiting differential settlements. In the field, the bearing layer elevation may vary; in that case, the driving resistance can serve as a guide in reaching consistently the same layer.

g) Future geotechnical research efforts should focus on studying the effect of pile setup and the effect of pile-soil interaction in pile groups on pile capacity. Establishing correlations between CPT measurements and pile resistances for local soils will also be beneficial.

h) Deep foundation projects should more often include static pile load testing. Besides allowing for the use of a lower factor of safety, more frequent performance of static load tests will help improve the knowledge of pile resistance in local soil types.

i) Pile load test data should be shared with the private geotechnical consultants who prepare the geotechnical report. Their involvement should continue also after the submission of the geotechnical report for the purpose of validating the analysis methods they used. This will assist in developing pile design knowledge that is suitable for local soils types. The creation of a public access database of pile load test results will be beneficial for private firms, INDOT engineers, and academic researchers.

j) There is the need to establish the framework and procedures that will allow effective interaction between the geotechnical consultant and the design consultant who are involved in Fast Track projects. 


\section{CHAPTER 3. STATIC METHODS FOR THE DETERMINATION OF PILE CAPACITY}

This chapter presents and compares methods proposed in the technical literature for the determination of the static unit resistances. The interviews presented in Chapter 2 revealed that there exists a lag between the methods currently used in pile design and more accurate methods that are the outcome of research efforts during the last decade. INDOT and private engineers currently use the methods recommended by the FHWA guidelines. The Geotechnical Manual of INDOT (INDOT 2005) recommends the use of Nordlund's and Tomlinson's methods for the calculation of unit resistances of displacement piles in "cohesionless" (sandy) and "cohesive" (clayey) soils, respectively. These methods are available in the popular pile design software DRIVEN. The methods recommended by the FHWA guidelines constitute the core of the computer programs used in design practice. Salgado et al. (2002) compared two new unit resistance calculation methods for open- and closed- ended pile piles with those commonly used by INDOT. It was suggested that the existing methods are excessively conservative and that significant savings can be achieved by using the new methods. The introduction of updated and improved equations for the calculation of pile resistances will lead to reduction in the cost of deep foundation work and enhance the reliability of the foundation design. The unit shaft and base resistances are the most important input parameters in dynamic analyses of pile driving since they play an important role in controlling the pile settlement per hammer blow. In addition, accurate and reliable equations are a prerequisite for the development of Load and Resistance Factor Design (LRFD) for pile foundations.

\subsection{Pile Design Process}

According to Salgado (2008), the design process includes the following steps:

a) Selection of piles over other types of foundation structures.

b) Selection of pile type based on local practice, constructability, economics, magnitude of structural loads, and other factors.

c) Examination of soil profile and decision on pile length. If there exists a soil layer with high strength/stiffness (e.g., stiff clay, dense sand, or rock) within reach, the pile length is chosen such that the pile tip is embedded in the competent layer at least 
two times the pile width; this is what would be commonly referred to as an endbearing pile. If a competent layer is not present at reachable depths $(30-40 \mathrm{~m})$, the pile is designed as a floating pile.

d) Decision on the pile cross-section based on static pile capacity calculations.

e) Selection of driving system in case of driven piles.

f) Specification of minimum pile length/minimum driving resistance to be reached during construction.

g) Specification of installation method for a drilled shaft based on soil profile and groundwater presence.

The goal of the static pile capacity calculations is the determination of the ultimate total resistance $Q_{\mathrm{ult}}$ of a single pile. $Q_{\mathrm{ult}}$ is expressed as the sum of the limit shaft resistance $Q_{\mathrm{sL}}$ and the ultimate base resistance $Q_{\mathrm{b}, \text { ult: }}$ :

$$
Q_{\text {ult }}=Q_{\mathrm{b}, \text { ult }}+Q_{\mathrm{sL}}
$$

The base resistance is expressed as

$$
Q_{\mathrm{b}, \mathrm{ult}}=q_{\mathrm{b}, \mathrm{ult}} A_{\mathrm{b}}
$$

where $q_{\mathrm{b}, \text { ut }}$ is the ultimate unit base resistance and $A_{\mathrm{b}}$ is the area of the pile base. For the calculation of $Q_{\mathrm{sL}}$, the part of the soil profile penetrated by the shaft is divided in $N$ sublayers:

$$
Q_{\mathrm{sL}}=\sum_{i=1}^{N} q_{\mathrm{sL}, \mathrm{i}} A_{\mathrm{s}, \mathrm{i}}
$$

where $q_{\mathrm{sL}, \mathrm{i}}$ is the limit unit shaft resistance along the part of the shaft intersecting the $i^{\text {th }}$ sublayer and $A_{\mathrm{s}, \mathrm{i}}$ is the corresponding shaft wall area. The division in sublayers generally follows the stratification as established through the field investigation, with individual soil layers being further subdivided into thinner sublayers to increase the accuracy of the computations. This is because the many variables used for the estimation of the unit shaft resistance, such as the effective stresses or the undrained shear strength, vary with depth. 
From previous paragraphs, it can be seen that there is a distinction between 'limit' and 'ultimate' resistance. The resistance (either for shaft or base) increases with increasing pile settlement but at a decreasing rate. Beyond a certain level of pile settlement, the resistance reaches a maximum value and remains constant. This maximum value constitutes the limit resistance. The pile settlement required for reaching the limit shaft resistance is relatively small (of the order of $1 \%$ of the pile width $B$ ). Hence, it is expected that $Q_{\mathrm{SL}}$ will be fully mobilized at the design limit state, except for a pile relying in an overwhelming way on shaft resistance. In contrast to the shaft resistance, the limit base resistance $Q_{b L}$ is mobilized only at large pile settlements, which can be in excess of $0.2 B$ in the case of sands (Fig. 3.2).

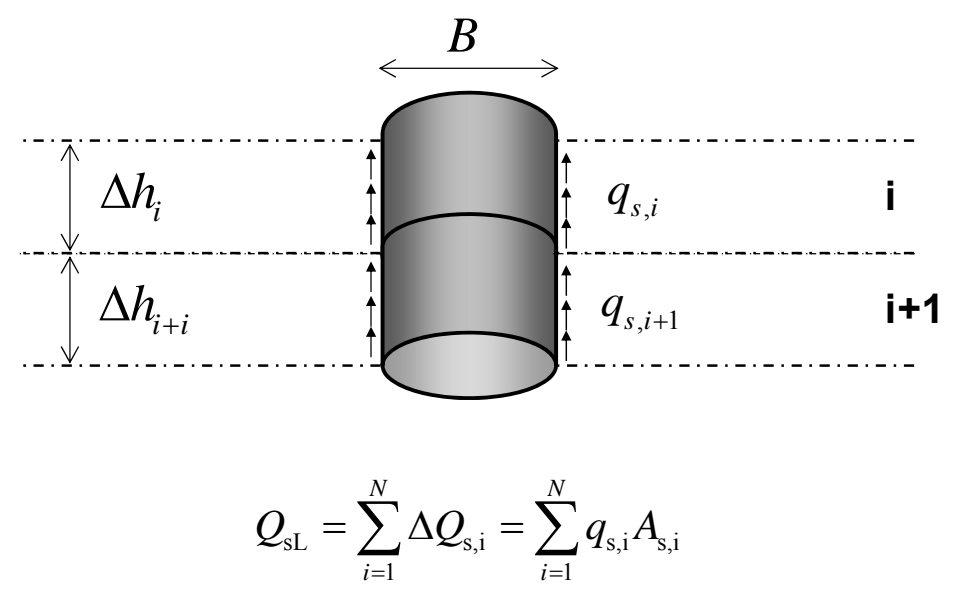

Figure 3.1. Division into sublayers for the calculation of the limit shaft capacity.

Common structures will have surpassed their ultimate limit state (there will have been structural damage) before the pile settlement levels required to reach $Q_{\mathrm{bL}}$ develop. Thus, pile design needs to be based on the ultimate base resistance $Q_{b \text {,ult, }}$ which is the base resistance value associated with the ultimate limit state. The pile settlement corresponding to the ultimate limit state is usually assumed to be equal to $10 \%$ of the pile width (Fig. 3.2). The unit base resistance is usually expressed as a net resistance (gross resistance less the weight of the pile), so that pile design is done by comparing the total pile capacity (eq. 3.1) with the pile head load (structural load). 


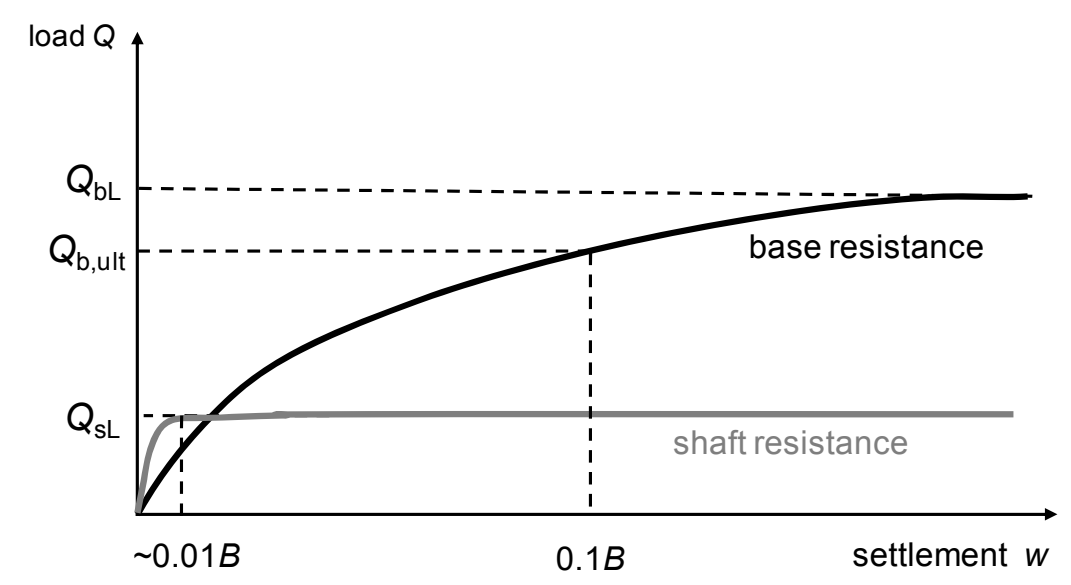

Figure 3.2. Mobilization of shaft and base resistances with pile settlement.

The static methods used for pile design are generally categorized into two types: a) methods based on soil properties and b) methods based on in situ tests. In soil-propertybased methods, the unit resistances are calculated using soil parameters readily available in typical geotechnical reports, such as the shear strength parameters $\left(\phi, s_{\mathrm{u}}\right)$, relative density $D_{\mathrm{R}}$, overconsolidation ratio $O C R$, and the plasticity index $P I$. In situ test data is not used directly into the property-based formulas, although some of the aforementioned parameters may be estimated based on in situ test data. In the methods based on in situ testing, the unit resistance is directly correlated to the SPT or CPT measurements.

The choice of the unit resistance formulas depends on the soil type (sandy vs. clayey) and the pile type (displacement vs. nondisplacement pile). The distinction between different pile types is necessary because displacement piles (driven or jacked piles) induce large changes in density and stress state in the surrounding soil during their installation. As a result, displacement piles generally exhibit larger unit shaft resistance than nondisplacement piles (drilled shafts). In addition, the ultimate base resistance of a displacement pile is expected to be greater than that of a nondisplacement pile installed in the same profile because the base resistance of displacement piles mobilizes with pile settlement at a higher rate than that of nondisplacement piles. 


\subsection{Property-based methods for unit base resistance in sand}

\subsubsection{Unit base resistance based on bearing capacity equation}

The unit limit base resistance of piles in sands has often been expressed in the same form as the bearing capacity equation for shallow foundations in sand (with the 'gamma' term neglected):

$$
q_{\mathrm{bL}}=N_{\mathrm{q}} q_{0} s_{\mathrm{q}} d_{\mathrm{q}}
$$

where $N_{\mathrm{q}}$ is the bearing capacity factor, $q_{0}$ is the overburden stress, and $s_{\mathrm{q}}$ and $d_{\mathrm{q}}$ are the shape and depth factors. Since the embedment of the base is several times the pile width and pile cross sections are typically approximately equidimensional, the shape and depth factors can be lumped into the bearing capacity factor:

$$
q_{\mathrm{bL}}=N_{\mathrm{qL}} \sigma_{\mathrm{v}}^{\prime}
$$

Similarly, the ultimate base resistance is expressed as:

$$
q_{\mathrm{b}, \mathrm{ult}}=N_{\mathrm{q}, \mathrm{ult}} \sigma_{\mathrm{v}}^{\prime}
$$

Unity is usually subtracted from $N_{\mathrm{q} \text {,ult }}$ in order to take into account that the unit base resistance is a 'net' resistance (pile weight deducted from pile capacity). However, this may not be necessary because of the large values that $N_{\text {qult }}$ takes in the case of sands.

Salgado (1995) showed that the $q_{\mathrm{b} \text {,utt }}$ increases nonlinearly and at a decreasing rate with increasing depth and, consequently, with increasing $\sigma_{v}^{\prime}$. Thus, $N_{\mathrm{q} \text {,ult }}$ decreases with increasing $\sigma_{\mathrm{v}}^{\prime}$. According to Fleming et al. (1992), $N_{\mathrm{q}, \mathrm{ult}}$ for driven piles varies in a way that can be approximated by

$$
N_{\mathrm{quult}}=0.136 \exp \left(0.182 \phi_{\mathrm{p}}\right)
$$

where $\phi_{p}$ is the peak friction angle of the soil. Eq. (3.7) is based on the solution by Berezantsev et al. (1961). Bolton's (1986) equation is used to calculate $\phi_{p}$ using an iterative 
procedure. Using a guess value for $\phi_{\mathrm{p}}$, an initial $N_{\mathrm{q} \text {,ult }}$ estimate is obtained from eq. (3.7). Then, the representative mean effective stress inside the bearing capacity mechanism is calculated using

$$
\sigma_{\mathrm{mp}}^{\prime}=\sigma_{\mathrm{v}}^{\prime} \sqrt{N_{\mathrm{q}, \mathrm{ult}}}
$$

Subsequently, $\sigma_{m p}^{\prime}$ is used in the Bolton (1986) correlation to obtain a new estimate on $\phi_{p}$ :

$$
\phi_{\mathrm{p}}=\phi_{\mathrm{c}}+3\left[\frac{D_{\mathrm{R}}}{100 \%}\left(Q-\ln \sigma_{\mathrm{mp}}^{\prime}\right)-R_{\mathrm{Q}}\right]
$$

If no other information is available for the parameters $Q$ and $R_{Q}$, the values 10 and 1 can be used, respectively. Using the new $\phi_{p}$ estimate, $N_{q, \text { ult }}$ is recalculated using eq. (3.7). If the old and new $N_{\text {q,ult }}$ estimates are not sufficiently close, additional iterations are performed until satisfactory convergence is reached. The computer program DRIVEN uses the approach of eq. (3.6) but does not calculate $N_{q}$ using Eqs. (3.7) through (3.9). The $N_{q, \text { ult }}$ values in DRIVEN (including the correction factor of Thurman 1964) are $40 \%$ to $50 \%$ smaller than those yielded by eq. (3.7) for the same $\phi_{p}$ value.

\subsubsection{Unit base resistance based on cone resistance estimate}

The limit and ultimate base resistance are related to each other through the factor $c_{\mathrm{b}}$ :

$$
q_{\mathrm{b}, \mathrm{ult}}=c_{\mathrm{b}} q_{\mathrm{bL}}
$$

As stated in a previous section, the most widely used criterion for the selection of the ultimate base resistance is the $0.1 B$ settlement criterion. Table 3.1 presents the values of $c_{b}$ recommended by Salgado (2008) and established by considering the $10 \%$ of $B$ criterion. We can see that, in the most recently proposed methods, $c_{b}$ is a decreasing function of the relative density. In addition, $c_{b}$ for displacement piles is substantially larger than that for drilled shafts and CFA piles, reflecting the fact that base resistance is mobilized at a much higher rate in the case of displacement piles. 
Experimental results and analytical solutions have shown that the limit base resistance $q_{\mathrm{b}}$ is approximately equal to the cone penetration resistance $q_{\mathrm{c}}$ (Lee and Salgado 1999, Salgado 2008). Therefore, the ultimate base resistance $q_{\mathrm{b}, \text { ult }}$ may be calculated in the absence of CPT data if we are able to obtain an estimate of the cone penetration resistance, and thus of the $q_{\mathrm{bL}}$, using soil properties as input. The cone penetration resistance is a function of the relative density $D_{R}$ and lateral effective stress $\sigma_{\mathrm{h}}$. According to Salgado and Prezzi (2007), $q_{\mathrm{bL}}$ may be calculated using

$$
q_{\mathrm{bL}}=1.64 p_{\mathrm{A}} \exp \left[0.1041 \phi_{\mathrm{c}}+\left(0.0264-0.0002 \phi_{\mathrm{c}}\right) D_{\mathrm{R}}\right]\left(\frac{\sigma_{\mathrm{h}}^{\prime}}{p_{\mathrm{A}}}\right)^{0.841-0.0047 D_{\mathrm{R}}}
$$

where $\phi_{c}$ is the critical friction angle expressed in degrees, $D_{R}$ is the relative density in percentage, and $p_{\mathrm{A}}$ is a reference stress $(100 \mathrm{kPa}=0.1 \mathrm{MPa} \sim 1 \mathrm{tsf})$.

Table 3.1. Summary of recommended $c_{b}$ values for calculating unit base resistance of different pile types (Salgado, 2008)

\begin{tabular}{|c|c|c|}
\hline Pile Type & $c_{\mathrm{b}}$ & Reference \\
\hline \multirow{3}{*}{$\begin{array}{c}\text { Displacement Piles and } \mathrm{H} \text { - } \\
\text { Piles }\end{array}$} & $0.35-0.5$ & Chow $(1997)^{\star}$ \\
\hline & 0.4 & Randolph (2003) \\
\hline & $1.02-0.0051 \mathrm{D}_{\mathrm{R}}(\%)$ & Foye et al. $(2006)^{*}$ \\
\hline $\begin{array}{c}\text { Nondisplacement Piles } \\
\text { (drilled shafts) and CFA } \\
\text { Piles } \diamond\end{array}$ & $0.23-\exp \left[-0.0066 \mathrm{D}_{\mathrm{R}}(\%)\right]$ & Salgado $(2004,2006) \dagger$ \\
\hline
\end{tabular}

Finally, it is customary to limit the ultimate unit base resistance of drilled shafts to a value of $5 \mathrm{MPa}$. This is done in order to account for any limitations that the design method may have (this does not apply to the equation in Table 3.1) and to provide additional safety margin against insufficient base clean up before pouring of the concrete. DRIVEN caps also the base capacity of displacement piles using the limit values suggested by Meyerhof (1976). This limiting values varies from 0.6MPa for $\phi_{p}=30^{\circ}$ to $35 \mathrm{MPa}$ for $\phi_{p}=44^{\circ}$. 
Figure 3.3 compares the $q_{\mathrm{b}, \text { ult }}$ values obtained from the methods by Fleming et al. (1992), Randolph (2003), Salgado (2006a), and the FHWA recommended method used in DRIVEN. The $q_{\mathrm{bL}}$ used in the calculations was calculated using eq. (3.11). The peak friction angle used in the FHWA method was calculated for triaxial compression conditions using the Bolton (1986) correlation with mean effective stress obtained based on the in situ (preinstallation) lateral effective stress. We see that, for the given example, the Fleming et al. (1992) method produces the largest $q_{\mathrm{b} \text {,ult }}$ values. The FHWA approach yields values that lie between the Randolph (2003) and Salgado (2006a) predictions. It should be noted that the $q_{\mathrm{b}, \text { ult }}$ values of the FHWA approach were controlled by the limiting values of Meyerhof (1976).

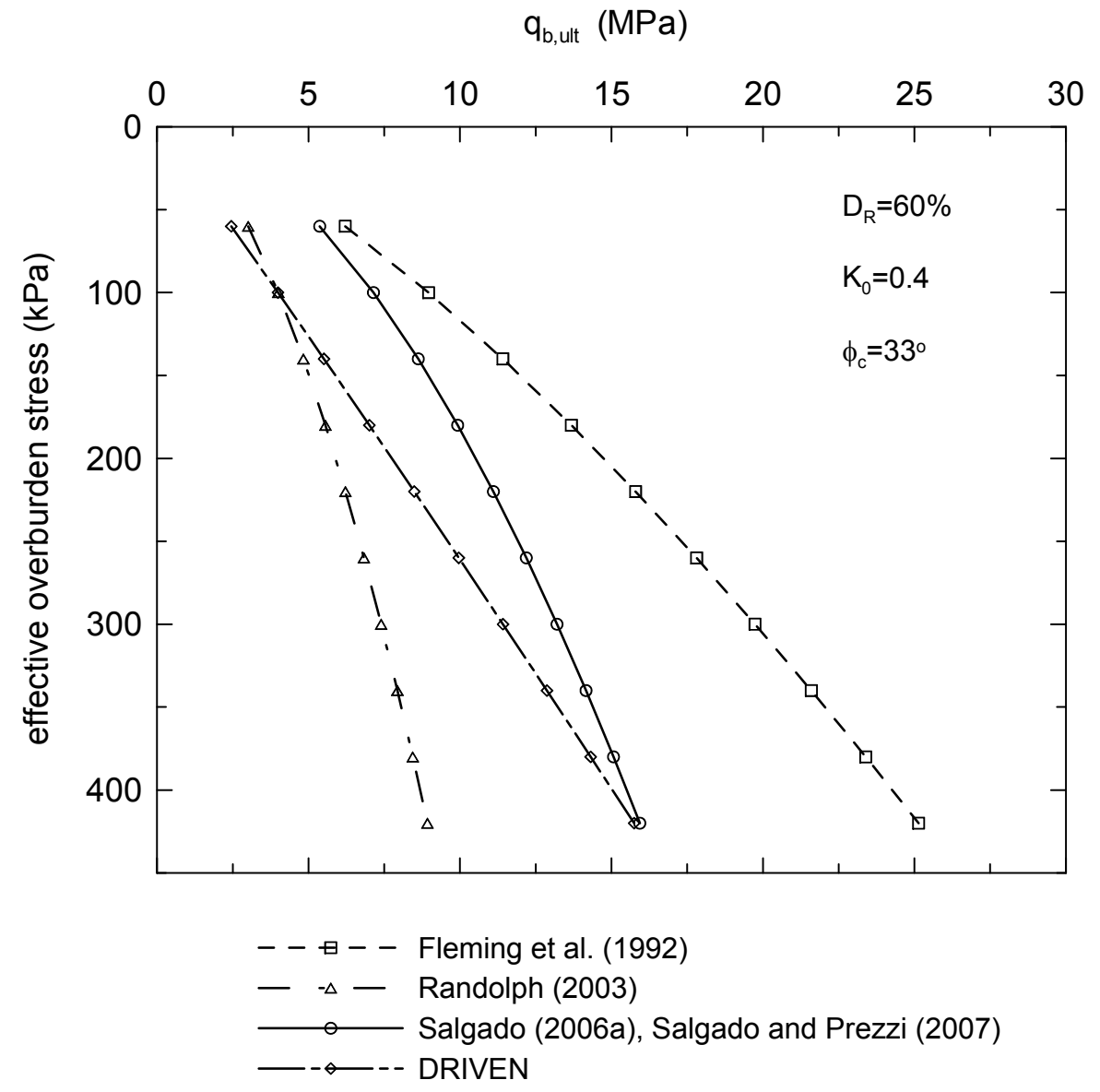

Figure 3.3. Comparison of ultimate base resistance values for displacement piles in sand from different soil-property-based methods. 


\subsection{Property-based methods for unit shaft resistance of nondisplacement piles in sand}

In frictional soils, the unit limit shaft resistance is the shear strength at the pile-soil interface due to friction. This shear stress is expressed as

$$
q_{\mathrm{sL}}=\sigma_{\mathrm{h}}^{\prime} \tan \delta
$$

where $\sigma_{\mathrm{h}}^{\prime}$ is the effective normal stress acting on the shaft wall and $\delta$ is the friction angle mobilized along the pile soil interface. The stress $\sigma_{\mathrm{h}}$ is, for convenience, expressed as the product of the initial (geostatic) vertical stress $\sigma_{v 0}^{\prime}$ and a coefficient $K$ (the operative value of the lateral effective stress coefficient). Consequently, Eq. (3.12) is further expanded to

$$
q_{\mathrm{sL}}=K \sigma_{\mathrm{v} 0}^{\prime} \tan \delta
$$

This method is often called the $\beta$-method because the product $K \tan \delta$ is usually replaced in eq. (3.13) by the Greek letter $\beta$. Several researchers have proposed values or formulas for the parameters $K$ and $\delta$, based mostly on back-calculation from pile load test data. Recent numerical studies (Loukidis, 2006; Loukidis and Salgado, 2008) showed that $K$ depends on the relative density of the sand and the level of initial effect stresses. These two variables control the sand dilatancy. The more dilative the sand is (i.e., the lower the confining stresses and higher the relative density), the more the sand around the pile shaft tends to dilate. This dilation is partly constrained by the soil lying further away from the pile shaft, leading to the buildup of lateral effective stress and large $K$ values. The opposite is true for low relative density and high confining stresses, in which case there is little to no dilatancy. 


\subsubsection{Shaft resistance in sands according to Reese et al. (1976)}

Reese et al. (1976) performed a series of load test on instrumented drilled shafts installed predominantly in sandy soils. Because the surface of a drilled shaft is very rough, they proposed that $\delta$ should be set equal to the internal soil friction angle of the soil $\phi$. This is the peak soil friction angle as measured in direct shear or triaxial compression tests, or estimated from SPT data. The coefficient $K$ takes the value of 0.7 for drilled shaft penetration $L_{p}$ in sand less than $8 \mathrm{~m}, 0.6$ for $L_{p}$ between $8 \mathrm{~m}$ and $12 \mathrm{~m}$, and 0.5 for $L_{p}$ greater than $12 \mathrm{~m}$. The Reese et al. (1976) recommendations indirectly recognize the fact that, as the penetration depth and, consequently, the confining stresses increases, the lateral effective pressure coefficient $K$ decreases.

\subsubsection{Shaft resistance in sands according to Stas and Kulhawy (1984)}

Stas and Kulhawy (1984) examined the data from a large number of pile load tests and observed that $K$ decreases with depth $z$. Stas and Kulhawy (1984) attribute any variation of the value of $K$ with depth to a presumed variance of the overconsolidation ratio OCR with depth. They proposed that $K$ can be taken equal to the coefficient of lateral earth pressure at rest $K_{0}$. The $K_{0}$ is meant to be estimated from OCR and $\phi$ using the equation

$$
K_{0}=(1-\sin \phi) O C R^{\sin \phi}
$$

In the method, the OCR is estimated from SPT data and $\delta$ is assumed equal to the internal (peak) soil friction angle of the soil $\phi$ as estimated from SPT data with an upper limit of $40^{\circ}$.

\subsubsection{Shaft resistance in sands according to O'Neill and Reese (1999)}

This method is recommended by FHWA and is used in the commercial software SHAFT. O'Neill and Reese (1999) generally adopt the work of Stas and Kulhawy (1984), with the difference that $\phi$ is recommended to be taken as the critical state friction angle $\phi_{\mathrm{c}}$ because of the large shear strain developing inside the shear band surrounding the pile shaft at limit resistance conditions. O'Neill and Reese (1999) suggest the use of the Stas and Kulhawy (1984) method if knowledge of $K_{0}$ is available. If $K_{0}$ cannot be estimated reliably, they recommend using the equations for $\beta$ established by O'Neill and Hassan (1994): 


$$
\beta=1.5-0.245 z^{0.5}
$$

for sands with $\mathrm{N}_{\mathrm{SPT}} \geq 15$,

$$
\beta=\frac{N_{\mathrm{SPT}}}{15}\left(1.5-0.245 z^{0.5}\right)
$$

for sands with $\mathrm{N}_{\mathrm{SPT}}<15$ and

$$
\beta=2.0-0.15 z^{0.75}
$$

for gravelly sands or gravels. The depth $z$ from the ground surface to the point on the shaft where $q_{\mathrm{sL}}$ is to be calculated must be in meters; $\beta$ is not allowed to take values smaller than 0.25 . In addition, $\beta$ is not allowed to take values larger than 1.2 in the case of sands. For gravelly sands or gravels, the upper limit is increased to 1.8 .

For normally consolidated (NC) sands, eqs. (3.15) and (3.16) predict $\beta$ values that are substantially higher than those predicted using the methods by Reese et al. (1976) and Stas and Kulhawy (1984). The $K$ value back-calculated using eqs. (3.15) and (3.16) $(K=\beta / \tan \delta)$ is much larger than the $K_{0}$ of an $\mathrm{NC}$ sand (which is usually in the 0.4 to 0.5 range) and even larger than $K$ values that are suitable for displacement piles. Eqs. (3.15) and (3.16) are valid mostly for overconsolidated sands with $K_{0}$ roughly equal to 0.8 . As stated by O'Neill and Hassan (1994), the data used for developing eqs. (3.15) and (3.16) comes from pile load tests in the overconsolidated sand deposits of the Gulf Coast region.

\subsubsection{Shaft resistance in sands according to Loukidis and Salgado (2008)}

Loukidis and Salgado (2008) used finite element analysis coupled with advanced constitutive modeling in order to investigate the mechanics involved in the development of limit shaft resistance of nondisplacement piles in sand. The results of the finite element simulations showed that $\delta$ is in the $0.95 \phi_{\mathrm{c}}$ to $1.0 \phi_{\mathrm{c}}$ range $\left(\phi_{\mathrm{c}}\right.$ is the critical state friction angle under triaxial compression conditions). Based on the finite element analysis results, they proposed the following equation for the lateral pressure coefficient:

$$
K=\frac{K_{0}}{\exp \left[0.2 \sqrt{K_{0}-0.4}\right]} C_{1} \exp \left[\frac{D_{\mathrm{R}}}{100 \%}\left(1.3-0.2 \cdot \ln \left(\frac{\sigma_{\mathrm{v}}^{\prime}}{p_{\mathrm{A}}}\right)\right)\right]
$$


where $D_{\mathrm{R}}$ is the sand relative density (in percentage), $p_{\mathrm{A}}$ is a reference stress $(100 \mathrm{kPa})$. Eq. (3.18) captures effectively the dependence of shaft resistance on relative density and effective overburden stress. The coefficient $C_{1}$ takes the values 0.71 and 0.63 for angular and rounded sands, respectively. The finite element methodology used was validated against centrifuge data (Fioravante 2002, Colombi 2005). In the case of a normally consolidated sand (the most usual case), eq. (3.18) predicts $K$ to be equal to or slightly less than $K_{0}$ for a loose sand $\left(D_{R} \leq 45 \%\right)$ and in the $1.5 K_{0}$ to $2.7 K_{0}$ range for very dense sand $\left(D_{R}=90 \%\right)$. The predictions of (eq. 3.18) are in general agreement with the range obtained using Reese et al. (1976).

Figure 3.4 compares $\beta$ values yielded by the methods of Stas and Kulhawy (1984), O'Neill and Reese (1999), and Loukidis and Salgado (2008). The interface friction angle $\delta$ is assumed to be equal $\phi_{c}$ for the Loukidis and Salgado (2008) method and to $\phi_{p}$ for the Stas and Kulhawy (1984) method. The peak friction angle $\phi_{p}$ is calculated for triaxial compression conditions using the correlation by Bolton (1986) using mean effective confining stress obtained based on the in situ (pre-installation) lateral effective stress. Eq. (3.18) yields $K$ values that lie between the two extremes: the conservative predictions of the Stas and Kulhawy (1984) method $\left(K=K_{0}\right)$ and the unconservative predictions by eqs. (3.15) and (3.16). 


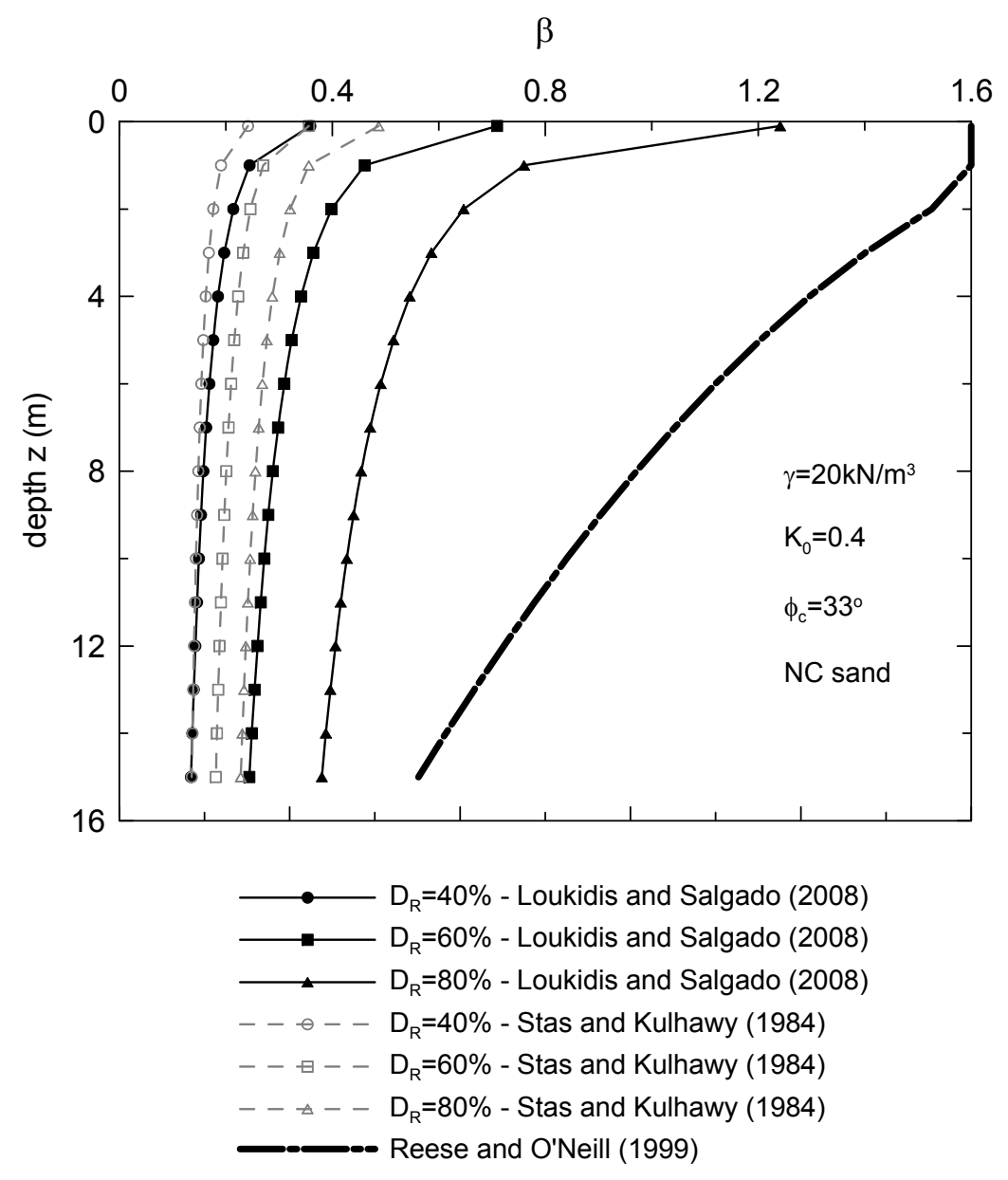

Figure 3.4. Comparison of values of $\beta$ from different soil-property-based methods for sands.

\subsection{Property-based methods for unit shaft resistance of displacement and partial- displacement piles in sand}

The unit shaft resistance of displacement piles (steel pipe piles, precast concrete piles) and partial displacement piles (H-piles, CFA) is calculated using the same basic formula (eq. 3.13) used for nondisplacement piles (i.e., the $\beta$-method). Displacement and partial displacement piles (with the exception of CFA) have generally smoother finished surfaces than drilled shafts, so $\delta$ is slightly smaller (especially for steel piles) than that used for drilled shafts. The recommended values of $\delta$ (Salgado, 2008) are shown in Table 3.2. 
Table 3.2. Values of $\delta$ for different pile types

\begin{tabular}{|c|c|}
\hline Pile Type & $\delta$ \\
\hline Steel & $0.85 \phi_{\mathrm{c}}$ \\
\hline Precast concrete & $0.95 \phi_{\mathrm{c}}$ \\
\hline CFA & $\phi_{\mathrm{c}}$ \\
\hline
\end{tabular}

The values of $K$ vary depending on the pile installation method. Based on Fleming et al. (1992), K can be calculated using

$$
K=0.02 N_{\mathrm{q}, \mathrm{ult}}
$$

where $N_{\text {q,ult }}$ is calculated using eq. (3.14) at the corresponding point along the pile shaft. Foye et al. (2006) established a correlation between $q_{\mathrm{b}, \mathrm{ult}}$ and $q_{\mathrm{sL}}$ :

$$
q_{\mathrm{sL}}=0.02 \tan \delta q_{\mathrm{b}, \mathrm{ult}}=0.02 \tan \delta\left[1.02-0.0051 D_{\mathrm{R}}\right] q_{\mathrm{bL}}
$$

where $q_{\mathrm{bL}}$ can be calculated from eq. (3.11) and $D_{\mathrm{R}}$ is in percent. Eq. (3.20) results in $K$ values that range from $K_{0}$ for very loose sand at high confining stresses to $5 K_{0}$ for dense sand at low confining stresses. These values are generally 1.5 to 2.5 times greater than the corresponding $K$ values for drilled shafts predicted by eq. (3.18).

The program DRIVEN uses the method proposed by Nordlund (1979). Nordlund (1979) provides charts for the determination of the ratio $\delta / \phi$ as a function of the pile type and the volume of displaced soil, with $\phi$ presumably being the peak friction angle estimated from SPT data (Peck, Hanson and Thornburn, 1974). In addition, $\sin \delta$ is used instead of $\tan \delta$ in eq. (3.13), $\delta$ decreasing with decreasing volume of displaced soil. The $K$ values proposed by Nordlund (1979) range from 0.7 to 4.3 (roughly $1.6 K_{0}$ to $9.6 K_{0}$ for NC sand) depending on the $\phi$ and the volume of displaced soil. Recommended $K$ values for CFA piles lie in the 0.9 to 1.0 range (Salgado 2008). Finally, for $\mathrm{H}$-piles, $K$ can be taken as $75 \%$ of the value of $K$ corresponding to a full displacement pile. 


\subsection{Property-based methods for unit base resistance of piles in clay}

The limit base resistance can be expressed using the bearing capacity equation for shallow foundations in clay:

$$
q_{\mathrm{bL}}=5.14 s_{\mathrm{u}} d_{\mathrm{c}} s_{\mathrm{c}}
$$

where $s_{u}$ is the undrained shear strength, $d_{c}$ is the depth factor and $s_{c}$ is the shape factor. Because the embedment ratio (penetration depth over pile width) in the case of piles is very high and because the cross section of the piles is symmetric with respect to both axis, the depth and shape factors take constant values and thus can be lumped inside a combined bearing capacity factor $N_{\mathrm{c}}$ :

$$
q_{\mathrm{bL}}=N_{\mathrm{c}} s_{\mathrm{u}}
$$

The most common value used for $N_{c}$ is 9 . This is the $N_{c}$ value for displacement piles considered in the program DRIVEN. Recent research employing advanced analysis methods (large strain finite element analysis, limit analysis, rigorous method of characteristics) reveals that the exact $N_{\mathrm{c}}$ value lies in the 10 to 12.5 range (Hu et al., 1999; Yu et al., 2000; Martin, 2001; Salgado et al., 2004). For piles in clayey soil, the ultimate base resistance based on the $10 \%$ of $B$ criterion is only slightly smaller than the limit base resistance for both displacement and nondisplacement piles (Table 3.3). In some cases, a pile in clay could reach $q_{\mathrm{bL}}$ in less than $0.1 \mathrm{~B}$ settlement. This is because the base resistance in clays increases with pile settlement at a higher rate than in sands.

Table 3.3. Summary of recommended design equations for calculating unit base resistance of different pile types (Salgado, 2008)

\begin{tabular}{|c|c|c|}
\hline Pile Type & $\mathbf{q}_{\mathrm{b}, \mathrm{ult}}$ & Reference \\
\hline $\begin{array}{c}\text { Nondisplacement piles (drilled } \\
\text { shafts) and } \\
\text { CFA piles }\end{array}$ & $9.6 \mathrm{~s}_{\mathrm{u}}$ & $\begin{array}{c}\text { Hu and Randolph (2002) } \\
\text { Salgado (2006a) }\end{array}$ \\
\hline $\begin{array}{c}\text { Displacement piles } \\
\text { (pipe piles and H-piles) }\end{array}$ & $10 \mathrm{~s}_{\mathrm{u}}$ & Salgado (2006a) \\
\hline
\end{tabular}

* Use the gross cross-sectional area of the base in the calculation of the total base resistance for soft and stiff clays 
O'Neill and Reese (1999) also consider $N_{\mathrm{c}}=9$ for drilled shafts, but only if $s_{\mathrm{u}} \geq 96 \mathrm{kPa}$, which usually implies overconsolidated clays. For smaller undrained shear strength values, they specify $N_{\mathrm{c}}=8$ and 6 for $s_{\mathrm{u}}$ roughly equal to $50 \mathrm{kPa}$ and $25 \mathrm{kPa}$, respectively. The departure from $N_{\mathrm{c}}=9$ for normally consolidated (NC) clays is most likely due to the use of an ultimate base resistance criterion that is based on a settlement less than $10 \%$ of $B$ and closer to $5 \%$ of $B$ (Salgado, 2008).

\subsection{Property-based methods for unit shaft resistance of piles in clay}

The shaft capacity in clays is more important than in sands because the ratio of base resistance to shaft resistance is less for clays than for sands. The unit limit shaft resistance in clays is expressed as the product of the undrained shear strength $s_{u}$ and a multiplication factor $\alpha$ (for this reason, this method is known as the $\alpha$-method):

$$
q_{\mathrm{sL}}=\alpha s_{\mathrm{u}}
$$

Research discovered early on that the parameter $\alpha$ is also a decreasing function of the $s_{\mathrm{u}}$. In essence, $q_{\mathrm{sL}}$ is a nonlinear function of $s_{\mathrm{u}}$. O'Neill and Reese (1999) proposed the following set of equations:

$$
\begin{array}{ll}
\alpha=0.55 & \text { for } \quad s_{\mathrm{u}} / p_{\mathrm{A}} \leq 1.5 \\
\alpha=0.55-0.1\left(s_{\mathrm{u}} / p_{\mathrm{A}}-1.5\right) & \text { for } 1.5<s_{\mathrm{u}} / p_{\mathrm{A}}<2.5 \\
\alpha=0.45 & \text { for } 2.5 \leq s_{\mathrm{u}} / p_{\mathrm{A}}
\end{array}
$$

Eq. (3.24) constitute the FHWA recommendations for drilled shafts. For displacement piles, FHWA recommendations adopt the method proposed by Tomlinson (1980). According to Tomlinson (1980), $\alpha$ depends on $s_{u}$, the ratio of the pile penetration depth to the pile width, and the morphology of the soil profile (e.g., whether the main clay layer is overlain or not by a superficial layer of sand or soft clay). The method is given in the forms of charts or tables. 
Table 3.4. Summary of recommended $\alpha$ values for calculating limit shaft resistance of different pile types (Salgado, 2008)

\begin{tabular}{|c|c|c|}
\hline Pile Type & $\alpha$ & Reference \\
\hline $\begin{array}{c}\text { Nondisplacement } \\
\text { piles (drilled shafts) } \\
\text { and } \\
\text { CFA piles }\end{array}$ & $\begin{array}{l}0.4\left[1-0.12 \ln \left(\frac{s_{u}}{p_{A}}\right)\right]_{*} \\
\text { used for } 3 \leq O C R \leq 5+\end{array}$ & Salgado (2006a) \\
\hline $\begin{array}{l}\text { Displacement piles } \\
\text { (pipe piles and } \mathrm{H}- \\
\text { piles) } \diamond\end{array}$ & $\alpha=\left\{\begin{array}{l}\left(\frac{s_{u}}{\sigma_{v}^{\prime}}\right)_{N C}^{0.5}\left(\frac{s_{u}}{\sigma_{v}^{\prime}}\right)^{-0.5}, \text { for } \frac{s_{u}}{\sigma_{v}^{\prime}} \leq 1 \\
\left(\frac{s_{u}}{\sigma_{v}^{\prime}}\right)_{N C}^{0.5}\left(\frac{s_{u}}{\sigma_{v}^{\prime}}\right)^{-0.25}, \text { for } \frac{s_{u}}{\sigma_{v}^{\prime}}>1\end{array}\right.$ & $\begin{array}{l}\text { Randolph and Murphy } \\
\qquad(1985), \\
\text { API (1993) }\end{array}$ \\
\hline
\end{tabular}

Values for $\alpha$ recommended by Salgado (2008) are shown in Table 3.4. The equation for drilled shafts in Table 3.4 (Salgado, 2006a) yields values that are always conservative compared to those yielded by the method of O'Neill and Reese (1999). This is because the equation by Salgado (2006a) was developed based on analytical consideration of a slightly to moderately overconsolidated clay. According to the equation by Randolph and Murphy (1985), which is also adopted by the American Petroleum Institute (API, 1993), $\alpha$ is a decreasing function of the overconsolidation ratio (OCR). The parameter $\alpha$ is equal to 1.0 as long as the clay is normally consolidated (NC). This is in contrast with predictions of the Tomlinson (1980) method, in which case $\alpha$ decreases with increasing $s_{\mathrm{u}}$ independently of the OCR. The Tomlinson (1980) method generally yields smaller values for $\alpha$ than the Randolph and Murphy (1985) equation, especially in the case of large effective confinement (large depths). 


\subsection{Methods based on in situ testing}

\subsubsection{CPT-based methods}

Methods based on in situ testing (CPT or SPT) rely on direct correlations between in situ test data performed prior to pile installation and pile capacity measured in pile load tests. There are strong similarities between the mechanics of the CPT and that of axial pile loading. Hence, CPT data can be effectively used to directly estimate $q_{\mathrm{b} \text {,ult }}$ and $q_{\mathrm{sL}}$. Cone penetration resistance $q_{c}$ can be considered to be roughly equal to the limit base resistance of displacement piles (Salgado 2008). The cone penetrometer can be seen as a small-scale model of a jacked pile. The main difference between the cone penetrometer and a pile with a larger base diameter is the size of the zone of soil influencing the base capacity. The general forms of the equations for the estimation of $q_{\mathrm{b} \text {,ult }}$ and $q_{\mathrm{sL}}$ using CPT results are:

$$
q_{\mathrm{b}, \mathrm{ult}}=c_{\mathrm{b}} q_{\mathrm{c}}
$$

and

$$
q_{\mathrm{sL}}=c_{\mathrm{s}} q_{\mathrm{c}}
$$

where $c_{\mathrm{b}}$ and $c_{\mathrm{s}}$ are constants that depend on the soil type and pile type. The $q_{\mathrm{c}}$ values used in eqs. (3.25) and (3.26) correspond to the depths for which calculations are done. For the base resistance, $q_{c}$ is calculated by averaging the representative cone resistance values over a distance of $1.5 B$ below the pile base and $1 B$ above the pile base, as shown in Figure 3.5. The soil profiles needs to be divided into subayers with similar soil properties and CPT values. For the calculation of the unit resistances, it is preferable to use the worst applicable CPT log as a conservative way to account for the spatial variability exhibited by sands. 


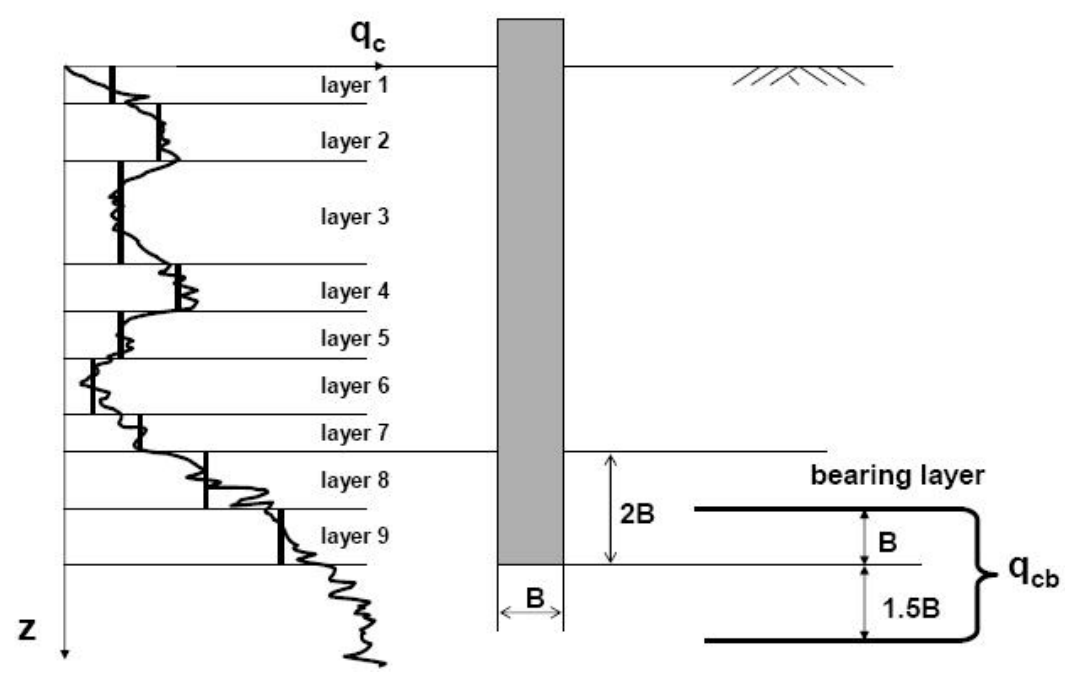

Figure 3.5. Typical layering scheme based on $q_{c}$ values along the pile shaft and recommended averaging of $q_{c}$ values in the bearing layer to find $q_{b L}$.

The multiplier $c_{b}$ can be calculated using the same equations used in the propertybased methods (e.g. Table 3.1). It generally depends on the pile type and the soil properties, particularly the relative density. Table 3.1 shows the most up-to-date values of $c_{b}$ for sand. The simplest approach is to use the constant vale of 0.4 proposed by Randolph (2003), although Salgado $(2006 a, b)$ found that $c_{b}$ decreases with increasing relative density.

CPT correlations for the shaft resistance are relatively scarce, especially for drilled shafts. The methods shown in Table 3.5 are based on research done by Aoki and Velloso (1975), Lopes and Laprovitera (1988), and Lee et al. (2003) for displacement piles. Aoki et al. (1978) also present numbers that can be used for the design of drilled shafts. There are no results in the literature for CFA piles yet.

Table 3.6 shows the $c_{b}$ values for clayey soils for the calculation of ultimate base resistance based on the $10 \%$ of $B$ criterion. The values proposed by Price and Wardle (1982), which are established based on pile load tests in stiff London clay, are considerably smaller than the state-of-the art range of 0.9-1.0. This is most likely because of the very small residual strength of London clay (Maksimovic, 1989). Table 3.6 illustrates the fact that, although state-of-art values are the most reliable, there are exceptions that can render them unconservative. Finally, the $c_{\mathrm{s}}$ values proposed for piles in clay are shown in Table 3.7. 
Table 3.5. Summary of $c_{\mathrm{s}}$ values for calculating limit shaft resistance of different pile types

\begin{tabular}{|c|c|c|}
\hline Pile Type & $\mathrm{C}_{\mathrm{s}}$ & Reference \\
\hline Displacement piles & $C_{s}=\left\{\begin{array}{l}0.004 \text { for clean sand } \\
0.0057 \text { for silty sand } \\
0.0069 \text { for silty sand with clay } \\
0.0080 \text { for clayey sand with silt } \\
0.0086 \text { for clayey sand }\end{array}\right.$ & $\begin{array}{l}\text { Aoki and Velloso (1975) } \\
\qquad *\end{array}$ \\
\hline $\begin{array}{l}\text { Nondisplacement } \\
\text { piles }\end{array}$ & $C_{s}=\left\{\begin{array}{l}0.0027 \text { for clean sand } \\
0.0037 \text { for silty sand } \\
0.0046 \text { for silty sand with clay } \\
0.0054 \text { for clayey sand with silt } \\
0.0058 \text { for clayey sand }\end{array}\right.$ & $\begin{array}{l}\text { Lopes and Laprovitera } \\
\qquad(1988)\end{array}$ \\
\hline $\begin{array}{l}\text { Open-ended pipe } \\
\text { piles }\end{array}$ & $C_{s}=\left\{\begin{array}{l}0.0015-0.003 \text { for } \mathrm{IFR} \leq 0.60 \\
0.0015-0.004 \text { for } 0.6<\mathrm{IFR} \leq 1\end{array}\right.$ & Lee et al. (2003) \\
\hline $\begin{array}{l}\text { Closed-ended pipe } \\
\text { pile }\end{array}$ & $c_{s}= \begin{cases}0.004-0.006 & \text { for } \mathrm{D}_{\mathrm{R}} \leq 50 \% \\
0.004-0.007 & \text { for } 50<\mathrm{D}_{\mathrm{R}} \leq 70 \% \\
0.004-0.009 & \text { for } 50<\mathrm{D}_{\mathrm{R}} \leq 90 \%\end{cases}$ & Lee et al. (2003) \\
\hline
\end{tabular}

*Aoki et al. (1978) recommend that the $c_{s}$-values be multiplied by 0.7 for Franki piles and by 0.5 for drilled shafts

Table 3.3.6. Summary of $c_{b}$ values for calculating ultimate base resistance of different pile

\begin{tabular}{|c|c|c|}
\hline Pile Type & $\mathbf{c}_{\mathbf{b}}$ & Reference \\
\hline Displacement piles & $0.9-1.0^{*}$ & State of the art \\
\hline Driven piles & $0.35^{\dagger}$ & Price and Wardle (1982) \\
\hline Jacked piles & $0.3^{\dagger}$ & Price and Wardle (1982) \\
\hline
\end{tabular}

*Applicable to soft and lightly OC clays

${ }^{\dagger}$ Applicable to stiff OC clays 
Table 3.7. Summary of $c_{s}$ values for calculating limit shaft resistance of different pile types

\begin{tabular}{|c|c|c|}
\hline Pile Type & $\mathbf{c}_{\mathbf{s}}$ & Reference \\
\hline Displacement piles & $c_{s}=\left\{\begin{array}{l}0.017 \text { for pure clay } \\
0.011 \text { for silty clay } \\
0.0086 \text { for silty clay with sand } \\
0.0080 \text { for sandy clay with silt } \\
0.0069 \text { for sandy clay }\end{array}\right.$ & $\begin{array}{c}\text { Aoki and Velloso (1975) } \\
*\end{array}$ \\
\hline Nondisplacement piles & $c_{s}=\left\{\begin{array}{l}0.012 \text { for pure clay } \\
0.011 \text { for silty clay } \\
0.010 \text { for silty clay with sand } \\
0.0087 \text { for sandy clay with silt } \\
0.0077 \text { for sandy clay }\end{array}\right.$ & $\begin{array}{c}\text { Lopes and Laprovitera } \\
\text { (1988) }\end{array}$ \\
\hline
\end{tabular}

${ }^{*}$ Aoki et al. (1978) recommend that the $c_{\mathrm{s}}$-values be multiplied by 0.7 for Franki piles and by 0.5 for drilled shafts

\subsubsection{Limit shaft resistance method of displacement piles in sand by Jardine et al. (1998)}

Jardine et al. (1998) developed a method for the calculation of $q_{\mathrm{sL}}$ for displacement piles in sand based on data from an extensive experimental program of instrumented field pile load tests. This method is called the Imperial College (IC) method and is CPT-based. The unit limit shaft resistance is given by

$$
q_{\mathrm{sL}}=\left[\frac{q_{\mathrm{c}}}{45}\left(\frac{\sigma_{\mathrm{v} 0}^{\prime}}{p_{\mathrm{A}}}\right)^{0.12}\left(\frac{B}{L-z}\right)^{0.38}+\Delta \sigma_{\mathrm{hd}}^{\prime}\right] \tan \delta
$$

where $L$ is the pile length below the ground surface, and $z$ is the depth from the ground surface. The first term inside the brackets represents the horizontal effective stress acting on the shaft at the end of the installation of the pile. The post-installation horizontal effective stress change $\Delta \sigma_{\text {hd }}$ is calculated using the following equation:

$$
\Delta \sigma_{\mathrm{hd}}^{\prime}=\frac{4 G \Delta t}{B}
$$

where $\Delta t$ is the change in shear band thickness during pile loading (taken equal to $0.02 \mathrm{~mm}$ ) and $G$ is the operational value of the shear modulus of the soil around the pile shaft. $G$ is given by 


$$
G=\frac{q_{\mathrm{c}}}{\left[0.0203+0.00125 \frac{q_{\mathrm{c}}}{\sqrt{p_{\mathrm{A}} \sigma_{\mathrm{v} 0}^{\prime}}}-1.216 \cdot 10^{-6} \frac{q_{\mathrm{c}}{ }^{2}}{p_{\mathrm{A}} \sigma_{\mathrm{v} 0}^{\prime}}\right]}
$$

The friction angle $\delta$ is the constant-volume (critical-state) friction angle mobilized along the shaft and can be estimated using Table 3.2. Eq. (3.27) is based on the mechanics involved in the installation of displacement piles.

\subsubsection{Limit shaft resistance method of displacement piles in sand by Randolph (2003)}

Randolph (2003) proposed a correlation between the lateral pressure coefficient $K$ to be used in eq. (3.13) and the cone penetration resistance $q_{\mathrm{c}}$ for displacement piles in sand:

$$
K=K_{\text {min }}+\left(K_{\text {max }}-K_{\text {min }}\right) \exp \left[-0.05 \frac{L-z}{B}\right]
$$

where $K_{\max }$ is taken as $1 \%$ to $2 \%$ of $q_{d} \sigma_{\mathrm{v} 0}^{\prime}(1.5 \%$ for practical purposes), $L$ is the pile length below the ground surface, and $z$ is the depth from the ground surface. $K_{\min }$ is the minimum possible $K$, which is encountered near the ground surface. $K_{\text {min }}$ takes values between 0.2 and 0.4 ( 0.3 for practical purposes). $K_{\max }$ is the $K$ value encountered close to the pile. Eq. (3.30) is in accordance with the mechanics involved in the installation of displacement piles. Driven and jacked piles are installed under action of multiple strokes, which cause several cycles of soil unloading and reloading. These cycles lead to the loss of effective confinement around the pile and consequently a reduction of limit shaft resistance. This phenomenon is called 'friction fatigue'. The upper part of the soil (close to the ground surface) experiences the most unloading-reloading cycles; accordingly, $K_{\min }$ is observed at shallow depths.

\subsubsection{Limit shaft resistance method of displacement piles in sand by Lehane et al. (2005)}

Lehane et al. (2005) combined experimental results from pile load tests in the field and in the centrifuge in order to develop a CPT-based method for the calculation of $q_{\mathrm{sL}}$ for displacement piles in sand. This method is called the University of Western Australia (UWA) method and is similar to the IC method. The unit limit shaft resistance is given by 


$$
q_{\mathrm{sL}}=\left[\frac{0.03 q_{\mathrm{c}}}{\sqrt{\max [(L-z) / B, 2]}}+\Delta \sigma_{\mathrm{hd}}^{\prime}\right] \tan \delta
$$

where $L$ is the pile length below the ground surface, and $z$ is the depth from the ground surface. As in the IC method, the first term inside the brackets represents the horizontal effective stress acting on the shaft at the end of the installation of the pile. The postinstallation change $\Delta \sigma_{\text {hd }}^{\prime}$ in the horizontal effective stress is again calculated using eq. (3.28). The change in shear band thickness change $\Delta t$ is taken to be equal to $0.02 \mathrm{~mm}$. The operational value of the shear modulus of the soil around the pile shaft is given by

$$
G=185 \frac{q_{\mathrm{c}}}{\left(\frac{q_{\mathrm{c}}}{\sqrt{p_{\mathrm{A}} \sigma_{\mathrm{v} 0}^{\prime}}}\right)^{0.75}}
$$

The friction angle $\delta$ is the constant-volume (critical-state) friction angle mobilized along the shaft, and can be estimated using Table 3.2. 


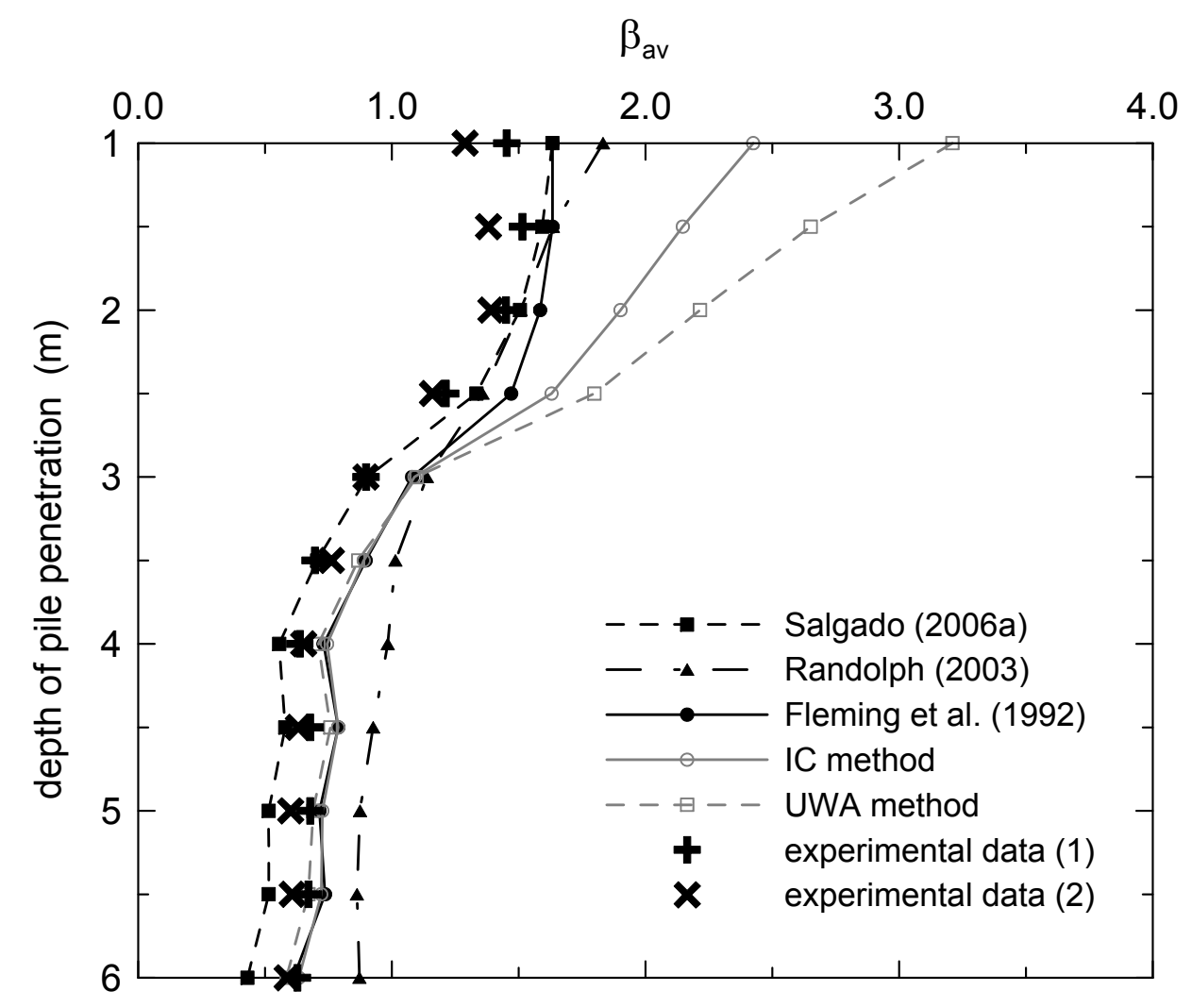

Figure 3.6. Comparison of average $\beta$ data from tests on jacked piles at the Labenne test site (Lehane et al. 1993) with predictions from several methods.

Basu et al. (2008) found that the IC method, the UWA method, the method by Randolph (2003), and the method by Salgado (2006a) yield predictions that are very close to each other and in close agreement with observed experimental data for both driven and jacked piles (Figure 3.6). This increases the confidence in the newly developed methods that attempt to be in accordance with the physics and mechanics of the pile resistance development.

\subsubsection{SPT-based methods}

While the SPT has no resemblance to pile loading, the SPT blow counts are affected by the same factors as cone penetration resistance $q_{\mathrm{c}}$. A relatively small number of SPT-based methods have been developed (e.g. Aoki and Velloso, 1975; Meyerhof, 1976,1983; Lopes and Laprovitera, 1988; Reese and O'Neill 1989). Similarly to the equations of the CPT- 
based methods, the unit base and shaft resistances are expressed as linear functions of the SPT blow count $N_{\mathrm{SPT}}$ :

$$
q_{\mathrm{b}, \mathrm{ult}}=n_{\mathrm{b}} N_{\mathrm{SPT}} p_{\mathrm{A}}
$$

and

$$
q_{\mathrm{sL}}=n_{\mathrm{s}} N_{\mathrm{SPT}} p_{\mathrm{A}}
$$

where $n_{\mathrm{b}}$ and $n_{\mathrm{s}}$ are parameters that depend on the soil type and pile type. O'Neill and Reese (1999) propose an SPT-based method for the determination of the unit ultimate base resistance of drilled shafts. According to O'Neill and Reese (1999), $n_{\mathrm{b}}$ is equal to 0.58 .

SPT-based methods are less reliable than CPT-based methods and soil-propertybased methods, especially for clayey soils. Therefore, the use of SPT-based methods is not recommended. In the absence of CPT data and in case the designer does not want to use a property-based method, it may be more reliable to convert SPT blow counts to cone penetration resistance and use a CPT-based method. This is possible because $q_{c}$ is well correlated to $N_{60}$ as both tests measure the resistance to penetration in the soil. The difference lies in that cone penetration is quasi-static, while the penetration of the SPT splitspoon occurs under dynamic conditions. Although such correlation should be used with caution, the combined error of the CPT-based method and that introduced by the SPT to CPT transformation may still be smaller than the error of SPT-based methods. The correlation by Robertson et al. (1983) gives the ratio $q_{d} /\left(p_{A} N_{60}\right)$ as a function of the mean particle size $D_{50}$. According to the Robertson et al. (1983) correlation, the ratio $q_{d} /\left(p_{A} N_{60}\right)$ varies from approximately 1 for clays to as high as 6 for coarse sands.

\subsection{Concluding remarks}

The methods recommended by the FHWA guidelines and used in pile design software generally yield conservative predictions compared to those by more recent and improved methods. A notable exception is the shaft resistance of drilled shafts, in which case the unit shaft resistance produced by the O'Neill and Reese (1999) equations is excessively large. In all other cases, the major benefit of using more recently proposed equations is not only the potential cost savings but also the enhanced reliability of the pile capacity prediction. This is 
because the recently proposed methods were developed based on the underlying physics, combining results from field tests, model tests, and analysis. The FHWA-recommended methods have focused almost exclusively on matching the prediction with the observed pile load test data, with little attention to the true physics and mechanics involved in the development of the pile resistances. In the case of piles in sands, most of the methods currently used in practice and implemented in pile design software fail to recognize two important facts: 1) the friction angle $\delta$ used in shaft resistance calculations is closely related to the critical-state friction angle $\phi_{c}$ and not to the peak friction angle $\phi_{p}$ and 2) the peak friction angle considered in the base resistance calculations is not the $\phi_{p}$ corresponding to the in situ soil state (i.e., that prior to pile installation) estimated from in situ tests but is a function of the soil state operative in the base failure mechanism at limit state conditions. Finally, although the superiority of methods that are based on CPT data over those based on SPT data has been recognized in the last two decades, CPT-based methods are absent from design software. CPTs should be performed in the field investigation of projects involving deep foundations whenever possible. 


\section{CHAPTER 4. ASSESSMENT OF DYNAMIC PILE ANALYSIS METHODS}

\subsection{Background}

The idea of using the observed pile response during driving for estimating the static pile capacity has been in existence for several decades. Given the cost and effort required for static pile load testing, the use of dynamic pile analysis is often attractive for verifying pile capacity. The observed pile response during driving can be interpreted using either empirical closed-form equations or wave equation analysis.

The empirical equations are based on a simple energy concept: the energy transmitted to the pile head by the hammer is equal to the work done by the total pile capacity for the observed pile head displacement (pile set) plus the energy dissipated inside the soil and the pile after a single blow. This can be written mathematically as:

$$
\eta W_{\mathrm{h}} h=R_{\mathrm{tot}}\left(s+s_{\mathrm{C}}\right)
$$

where $W_{\mathrm{h}}$ is the hammer weight, $\mathrm{h}$ is the hammer drop height, $\eta$ is the efficiency of the driving system, $R_{\text {tot }}$ is the total pile capacity, $s$ is the observed pile set, and $s_{\mathrm{c}}$ is an empirical constant (called the 'lost' set) expressing the energy dissipated in the pile and the soil. Eq. (4.1) has been the basis of many empirical dynamic equations. The equation is solved for $R_{\text {tot }}$ with the basic variable being the pile set $s$. The Engineering News (ENR) formula, which has been in use for more than a century, assumes a constant $s_{c}$ equal to $0.1 \mathrm{in}$. Another popular equation in the United States is Gates' formula:

$$
R_{\mathrm{tot}}=27 \sqrt{W_{\mathrm{h}} h}(1+\log s)
$$

where $W_{\mathrm{h}}$ and $R_{\text {tot }}$ are in kips, $h$ in feet, and $s$ in inches.

The widely used dynamic formulas have been criticized in many publications. Unsatisfactory performance predicting the pile capacity was documented in the Manual for Design and Construction of Driven Pile Foundations (Hannigan et al. 1996). Although 
empirical dynamic formulas are very easy to use, their predictions are characterized by considerable scatter and, in some case, bias (McVay et al. 2000). Consideration of empirical formulas nowadays offer little benefit to deep foundation design since the safety factor recommended when they are used exceeds the values of F.S. typically used when designing based on static methods (Salgado 2008). New attempts to improve the pile driving formulas (Paikowsky and Chernauskas 1992, Paikowsky et al. 1994) were shown to still suffer from drawbacks. This results from the complexities of the problem, which is in essence a highly nonlinear wave propagation problem involving complex physics and mechanics. Therefore, any potential advancements in the field of pile driving analysis lie in the use of the wave equation.

The first method employed in wave equation analysis for pile dynamics was the method of characteristics (De Josselin De Jong 1956). The method of characteristics is a semi-analytical method, in which the pile is treated as an undiscretized continuum. Early application of the method of characteristics in pile driving analysis assumed that all soil reactions are concentrated at the pile tip. Reaction along the shaft was later introduced as a fixed analytical function independent of displacement or velocity. These rather rough approximations were necessary in order to allow solution of the problem by integral transforms and derivation of the solution in the form of Fourier series. The software TNOWAVE developed by the research organization TNO in Netherlands uses the method of characteristics. The limitation of the method of characteristics in accounting for the continuous variation of shaft resistance with velocity and displacement is circumvented in this program by assuming that the shaft resistance is concentrated at given points along the pile shaft. These points are considered as internal boundaries to the problem. The method of characteristics provides the solution of the wave equation inside each pile segment defined by consecutive internal boundaries.

There are also a number of methods for pile dynamic analysis that are based on semi-analytical approaches other than the method of characteristics: (1) solutions for piles of semi-infinite length (Parola 1970, Van Koten et al. 1980, Warrington 1987, Deeks 1992, Parker et al. 1996), (2) solutions using the method of images (Glanville et al. 1938, Hansen and Denver 1980, Uto et al. 1985), (3) solutions by Fourier series (Wang 1988, Espinoza 1991), (4) solutions by Laplace transforms (Zhou and Liang 1996). The main disadvantage of the semi-analytical methods, including the method of characteristics, is that they involve complex mathematics that hinder the implementation of realistic soil reaction models. 
A major breakthrough in pile driving analysis was the work done by Smith in the late 1950s. Smith (1960) developed an entirely numerical method to analyze pile driving without the use of complex mathematics. The pile is discretized into a series of lumped masses connected with linear springs (Figure 4.1). The global system of equations of motion (dynamic equilibrium equations) was solved in the time domain by dividing the analysis time into small time increments. The numerical solution schemes most often used are the central difference methods and the Newmark $\beta$-method. Smith's approach is often called the 1dimensional approach since the effect of the surrounding soil mass is accounted for through reaction models consisting of springs, dashpots and sliders connected to each other in various combinations. Originally, the soil resistance at any point along the pile and at the pile tip was modeled by a linear-perfectly plastic spring-slider and a dashpot connected in parallel. Various researchers (Forehand and Reese, 1964, Lowery et al., 1969, Holloway et al. 1978, Briaud and Tucker, 1984) who studied Smith's 1-D analysis approach concluded that the method has the potential to become accurate and reliable method provided that limitations are addressed. The popularity of the method grew considerably, and in the course of the following years a number of improved soil reaction models were proposed.

The finite element method (FEM) has also been used in recent years for the simulation of the pile driving problem (e.g. Borja 1988, Mabsout and Tassoulas 1994, Mabsout et al. 1995). FEM has the advantage that, in contrast with the 1-D approach, the soil around the pile is treated as a continuum instead of being represented by springdashpot-slider reaction models. Among the available methods for pile driving analysis, the finite element method can produce the most accurate results provided that realistic and advanced constitutive models are used for modeling the soil and the analysis domain is properly discretized. In the case of large pile sets, a large strain formulation is also needed for the correct prediction of the development of limit base resistance. All these requirements result in very computationally expensive simulations, with runtimes of the order of several hours or days, even in the fastest computers. Therefore it is currently impossible to use FEM in routine pile dynamic analysis in design practice.

This chapter presents the soil reaction models used in 1-D dynamic analysis for pile driving that have been proposed so far. The purpose of this chapter is to indicate the advantages and disadvantages of this approach and identify potential points of improvement. Any advancements in terms of soil reaction modeling will be achieved through synthesis and modification of existing models. 


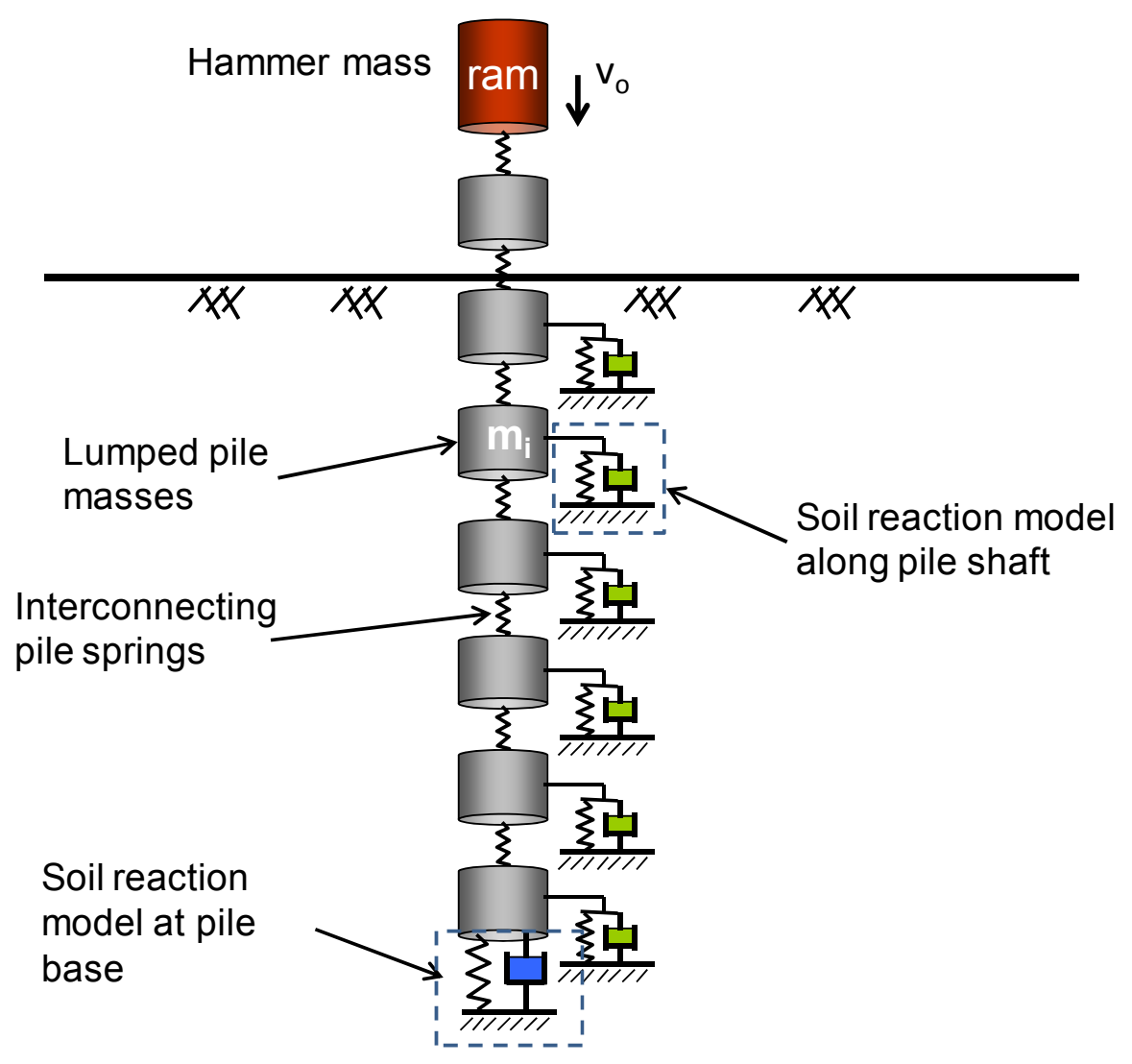

Figure 4.1. Smith's numerical model for dynamic pile analysis: lumped mass discretization and soil reaction models

\subsection{Mechanisms of soil reaction mobilization along pile shaft and base}

Pile driving is a highly nonlinear dynamic problem. Stress waves are transmitted from the pile to the soil, and there are also regions where the soil "fails". Driven piles (excluding those installed using vibratory hammers) penetrate the soil due to the hammer impact on the pile head. As a result, the motion of the pile and the cyclic loading in the soils is transient in nature. The soil in the immediate vicinity of the pile can store energy (elastic deformation) and absorb energy because of plastic and hysteretic dissipation. Plastic dissipation occurs in the highly strained zones adjacent to the pile in which soil undergoes post-failure plastic deformation. Hysteretic dissipation (hysteretic damping) originates from the nonlinear response of the soil even at the small or intermediate (pre-failure) strain range. The 
hysteretic damping of soils has been extensively studied in the context of earthquake geotechnical engineering. It is usually expressed in the form of the damping ratio, which represents the percentage of the input energy that is absorbed in the soils during a full stress cycle. The damping ratio increases with the magnitude of the cyclic shear strain, ranging from $0.005 \%$ to $30 \%$. Energy is also radiated in the far field (radiation damping). Research studies in the field of machine foundations have produced closed-form solutions for the radiation damping around pile shafts and under footings that may be used in pile driving analysis.

Figure 4.2a shows a schematic representation of the deformation and energy absorption around the pile shaft. As the pile moves downward, it induces shear stress in the soil along its shaft. Limit shaft resistance $q_{\mathrm{sL}}$ is reached with relatively small pile displacement. Under static conditions, the pile displacement $w_{\mathrm{p}}$ required to mobilize $q_{\mathrm{sL}}$ is of the order of $1 \%$ of the pile width $B$. Under dynamic conditions, $w_{\mathrm{p}}$ is smaller because of the short wave length of the shear waves radiating outwards from the pile shaft. A thin shear band is formed around the pile at the moment the limit shaft resistance is reached. All plastic deformation happens inside that shear band. The thickness of the shear band is proportional to the mean particle size of the soil. If the pile roughness is high, as in the case of precast concrete piles and drilled shafts, then slippage occurs entirely inside the sand, while for smoother pile surfaces (such as those of steel piles) slippage develops also between soil and pile shaft wall. In the rough interface case, the shear band thickness is approximately 5$20 D_{50}$ (Uesugi et al. 1988; Oda et. al 2004) for sands and of the order of $200 D_{50}$ for clays (Vardoulakis 2002). For the smoother pile surfaces, the shear band thickness in sands can be as low as $3 D_{50}$ (Uesugi et al. 1990). The soil outside the shear band remains in a "prefailure" state and undergoes predominantly cyclic vertical shearing. The magnitude of the cyclic shear stress reduces hyperbolically with radial distance from the shaft. The soil region closer to the pile (near field) absorbs most of the energy, with the remaining energy propagating to the far field (radiation damping). If the pile were perfectly rigid, only vertical shear waves (SV) would radiate from the pile shaft (at elevations not close to the ground surface or the pile base). In reality, compressive waves are also generated because the pile is deformable and the upper sections of the pile are set in motion earlier than the lower sections as the hammer pulse travels downwards. However, the effect of compressive waves is much less significant, and the vertical shear wave dominates the mode of deformation around the pile shaft. Because of the high velocity of pile motions and the large induced strain rates in the soil, the strength of the soil inside the plasticity zones is likely to 
be higher than under static conditions. Therefore, the limit shaft and base capacities are expected to be dependent on the pile velocity (rate effect). This means that, in addition to hysteretic damping and radiation damping, there will be energy absorption due to viscous damping inside the plastic zones.

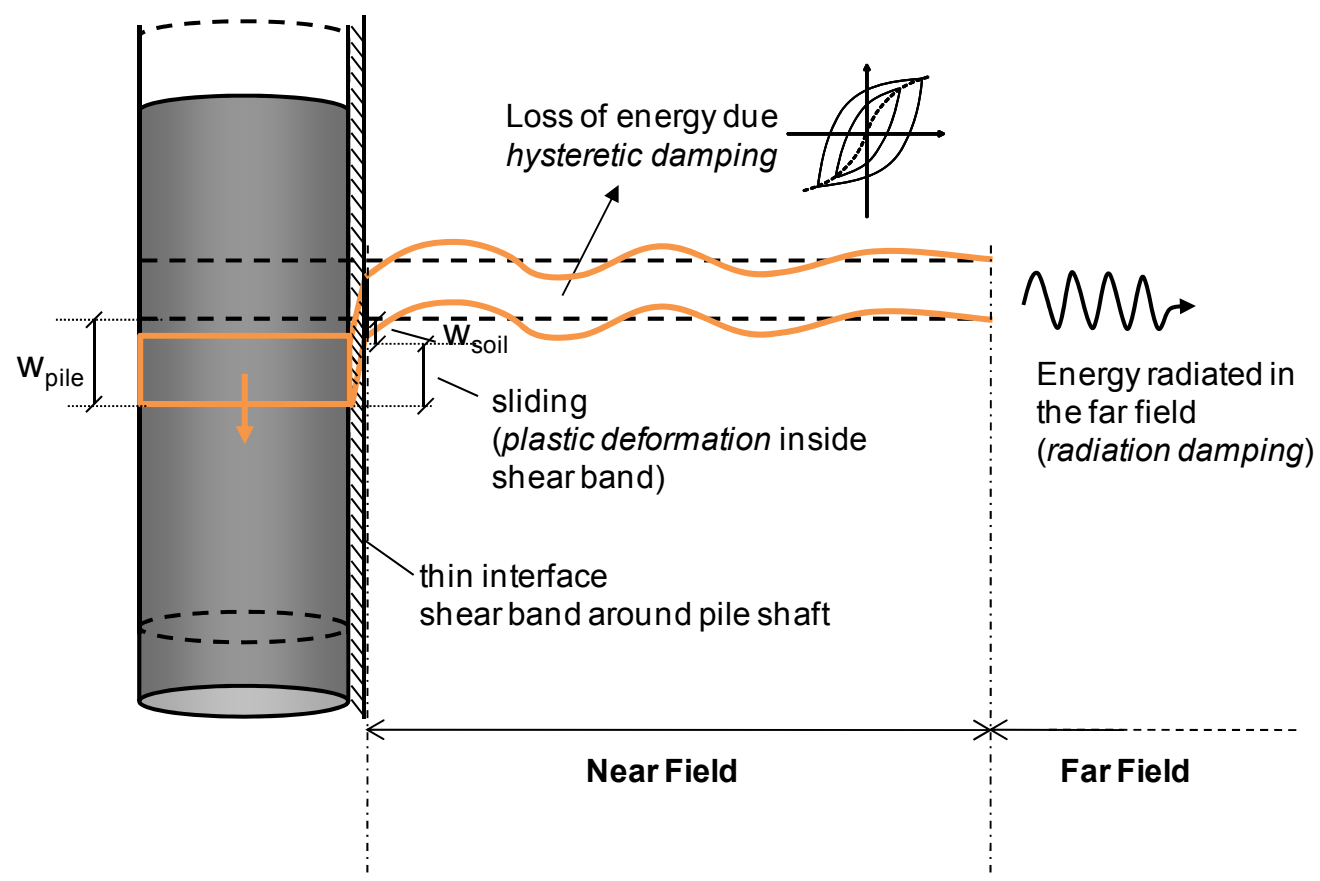

(a)

Bearing capacity mechanism (zones of diffused plastic deformation)

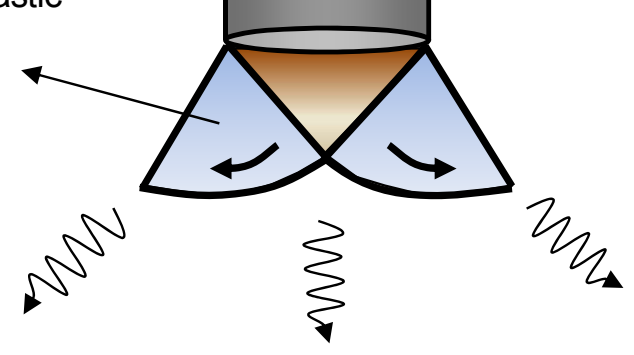

Loss of due to radiation damping and hysteretic damping)

(b)

Figure 4.2. Energy transmission and absorption and deformation mechanism in the soil during pile driving: a) around the pile shaft, b) at the pile base. 
In the case of the pile tip, the plastic deformation is diffused in a region (Figure 4.2b) that extends from the pile base to roughly 1 to 2 times the pile width. The plastic mechanism resembles strongly the bearing capacity mechanism of footings. Assuming that the pile is not fabricated or fitted with a conical tip, a 'trapped' or 'rigid' conical area is formed under the pile base. This area remains elastic and can be considered as an extension of the pile. The plastic deformation takes place in the fan zone that surrounds the 'rigid' conical area. The soil outside the plastic mechanism provides lateral reaction to the expansion and rotation of the fan zone. As shear and compressive waves propagate through the outer soil region, energy is lost due to hysteretic and radiation damping. Most of the energy radiated from the pile base travels downwards, but a certain nonnegligible amount of energy is also transmitted towards the ground surface.

\subsection{Soil reaction models}

\subsubsection{Smith (1960) shaft and base reaction models}

The soil reaction in Smith's model depends on both pile displacement $w_{\text {pile }}$ and pile velocity $\mathrm{d} w_{\text {pile }} / \mathrm{d} t$. It consists of a dashpot that is connected in parallel with a system of a linear spring and a plastic slider connected in series (Figure 4.3). The soil reaction in terms of stress on the pile shaft can be written as

$$
\tau_{\mathrm{s}}=\min \left(k_{\mathrm{s}} w_{\text {pile }}, q_{\mathrm{sL}}\right)+c_{\mathrm{s}} \dot{w}_{\text {pile }}
$$

where $k_{\mathrm{s}}$ is the spring stiffness coefficient, $c_{\mathrm{s}}$ is the dashpot coefficient, and $q_{\mathrm{sL}}$ is the unit limit shaft resistance. The spring stiffness is given by the following equation

$$
k_{\mathrm{s}}=\frac{q_{\mathrm{SL}}}{Q_{\mathrm{S}}}
$$

where $Q_{\mathrm{S}}$ is an input parameter called the soil 'quake', which has units of length. The quake represents the displacement at which perfect plasticity starts. If the pile displacement $w_{\text {pile }}$ exceeds the quake, then slider motion is activated and spring deformation stops. The dashpot coefficient is given by equation 


$$
c_{\mathrm{S}}=J_{\mathrm{s}} q_{\mathrm{sL}}
$$

where $J_{s}$ is a damping input parameter. Similarly for the base, we have soil reaction in terms of force expressed as

$$
R_{\mathrm{b}}=\min \left(K_{\mathrm{b}} w_{\text {pile }}, R_{\mathrm{bL}}\right)+C_{\mathrm{b}} \dot{w}_{\text {pile }}
$$

where $R_{\mathrm{bL}}$ is the limit base resistance. The spring and dashpot coefficients are given by the following equations, respectively:

$$
\begin{gathered}
K_{\mathrm{b}}=\frac{R_{\mathrm{bL}}}{Q_{\mathrm{b}}} \\
C_{\mathrm{b}}=J_{\mathrm{b}} R_{\mathrm{bL}}
\end{gathered}
$$

The soil quake and damping constants proposed are not standard soil parameters. They are empirical constants determined from back-analyses of pile driving records and pile load tests. Smith (1960) proposed the values $Q_{s}=Q_{b}=2.5 \mathrm{~mm}(0.1 \mathrm{in}), J_{s}=0.16 \mathrm{~s} / \mathrm{m}(0.05 \mathrm{~s} / \mathrm{ft})$, and $J_{b}=0.492 \mathrm{~s} / \mathrm{m}(0.15 \mathrm{~s} / \mathrm{ft})$. Updated empirical damping coefficients were later proposed for sands and clays based on laboratory impact tests (e.g., Forehand and Reese, 1964; Coyle and Gibson, 1970; Litkouhi and Poskitt, 1980; Liang and Sheng, 1992), and correlations with soil type (e.g. Likins et al., 1992; Paikowsky et al., 1994) or SPT data (e.g. Liang 2000) were established. Generally, the $J$ values for clays and silts are higher than those for sands. This is apparently due to the higher-viscosity cohesive soils. However, the proposed $J$ and $Q$ values exhibit a large scatter, making it difficult to develop reliable correlations. Aoki and de Mello (1992) found that $J$ and $Q$ also vary not only with the soil properties and type but also with the level of the hammer energy.

There are some inconsistencies of the Smith (1960) models with the mechanics of pile driving (described in the previous section). Due to the connectivity of the model components (Fig. 4.3), the dashpot is always active, providing the same amount of damping before and after sliding. The model does not distinguish between hysteretic, radiation and 
viscous damping, lumping all of them into a universal viscous damping that is proportional to the static limit resistance. It is known that radiation damping is a function of the soil stiffness, not its strength. Moreover, laboratory testing has shown that the actual viscous damping in the plastic zone is not proportional to the static soil strength but a power function of it. These limitations of the original Smith (1960) model have been identified early on by researchers, and efforts were subsequently made to propose models that reflect reality more closely.

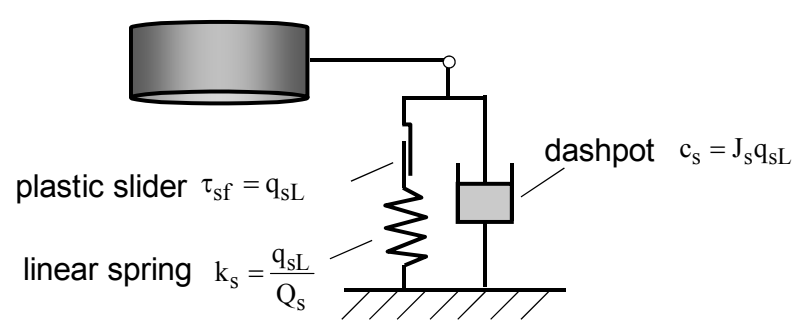

(a)

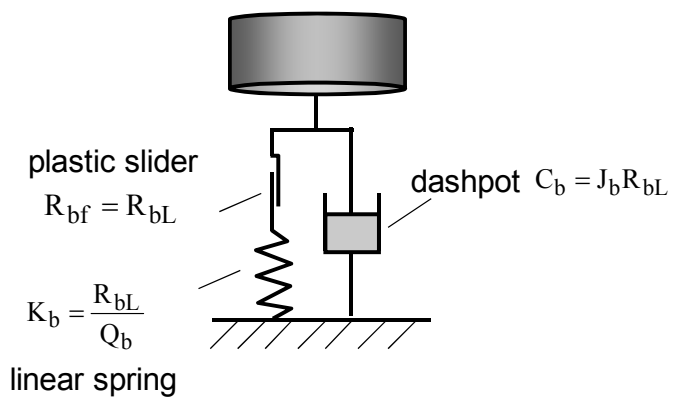

(b)

Figure 4.3. Smith's reaction models: (a) for pile shaft and (b) for pile base.

\subsubsection{Base reaction model based on Lysmer's analog}

Lysmer and Richart (1966) derived a closed-form solution for the motion of a circular rigid footing on the surface of an elastic half-space subjected to vertical transient load. Their solution gives the total reaction of the soil acting at the footing base as the sum of two components: a spring reaction (displacement-dependent) and a dashpot reaction (velocitydependent). The spring stiffness is the same as that of the static solution:

$$
K_{\mathrm{b}}=\frac{4 G R}{1-v}
$$

where $R$ is the radius of the circular area, $G$ is the elastic soil shear modulus, and $v$ is the Poisson's ratio of the soil. If the base area has an arbitrary shape (but is not very elongated), a circular area can be used to approximate the base area (the radius of the equivalent circular area is then used in calculations). The dashpot coefficient is given as 


$$
C_{\mathrm{b}}=\frac{3.4 R^{2}}{1-v} \rho V_{s}=\frac{3.4 R^{2}}{1-v} \sqrt{\rho G}
$$

where $\rho$ is the soil density and $V_{\mathrm{s}}$ is the shear wave velocity of the soil. The dashpot in Lysmer's model represents the radiation damping, i.e., the energy loss due to propagation of shear, compressive and Rayleigh waves in the elastic half-space. Lysmer's solution does not depend on the frequency of vibration. Lysmer's analog is adequately accurate for a linear elastic soil and impact type loading (highly transient response). It is also accurate for steady-state vibration as long as the footing motion frequency is not very high.

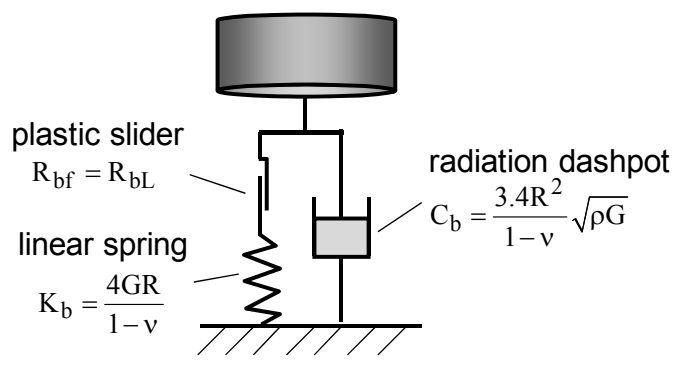

Option 1

(a)

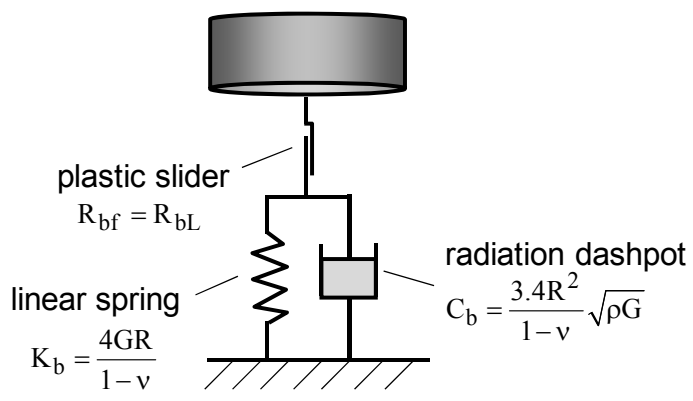

Option 2

(b)

Figure 4.4. Base models following Lysmer's analogue: a) radiation dashpot always active and b) radiation dashpot ceases to act after onset of slippage.

Lysmer's analogue has been used extensively in the field of machine foundations. Because soils are elastic only in the small-strain range, the appropriate value for the shear modulus is $G_{\max }$ (maximum or small-strain shear modulus) although researchers often suggest using degraded secant (equivalent) shear modulus values corresponding to shear stress amplitudes representative of the given problem in order to account for soil nonlinearity. However, equivalent $G$ values are hard to determine since the shear strain amplitude varies with distance from the source and with time (transient loading). Moreover, the equation for the dashpot coefficient representing radiation damping is rigorous only when $G_{\max }$ is used as the shear modulus (predominantly elastic deformation). 
Lysmer's analog coefficients can replace Smith's spring and dashpot coefficients (eq. 4.7 and 4.8), as shown in figure Fig. 4.4a (Option 1), thus allowing direct association of the base stiffness and damping with actual soil properties (elastic parameters and soil density). The model shown in Fig. 4.4a was used by Simons and Randolph (1985). Researchers have argued that no waves are transmitted to the soil after the base plastic mechanism has been fully formed, and thus the plastic zone is decoupled from the rest of the soil medium. Based on this consideration, some have proposed placing the slider outside the springdashpot system, as shown in Fig. 4.4b (Option 2). In that case, the dashpot does not contribute to the soil reaction after base capacity is reached. Since no tension can be transferred from the soil to the pile in reality, the soil reaction at the pile tip is not allowed to take negative values. Instead of tensile force developing at the base, a gap is formed between the pile and soil and the reaction there is zero (with the possible exception of a small tensile strength due to suction in the case of saturated clays). Positive (compressive) forces start developing again only when the gap closes in the course of the analysis.

Such models reduce significantly the empiricism of Smith's model but still miss certain aspects of the response mechanisms of the soil. These are the soil nonlinearity and the corresponding hysteresis damping and the rate dependence of the soil strength inside the failure mechanism on the strain rate (viscous damping). As a result, the models shown in Fig. 4.4 highly overestimate the base stiffness. Deeks and Randolph (1995) have also shown that the model in Fig. 4.4a overestimates the peak dynamic reaction, while that in Fig. 4.4b underestimates it.

\subsubsection{Shaft reaction model by Simons and Randolph (1985)/Randolph and Simons (1986)}

Randolph and Simons (1986) proposed a soil reaction model for the pile shaft that has input parameters with clear physical meaning. The model consists of two parts (Fig 4.5): 1) a spring and a dashpot (representing radiation damping) connected in parallel and 2) a plastic slider and a second dashpot (soil viscosity) connected in parallel. The two parts are in turn connected in series. The second part represents the shear band surrounding the pile shaft and the first part represents the rest of the soil, which has not reached a fully plastic state.

The spring and dashpot constants of the first part are based on the solution by Novak et al. (1978). Novak et al. (1978) derived a close-form analytical solution for the soil reaction acting on the shaft of a vertically vibrating, rigid, infinitely long pile by assuming a thin soil disk. The solution is rigorous for an elastic soil and steady-state pile motion. 
According to the Novak et al. (1978) analytical solution, the complex reaction of an elastic soil disk acting on the harmonically oscillating pile shaft is

$$
\tau_{\mathrm{s}}=\left(k_{\mathrm{s}}+i c_{\mathrm{s}}\right) w_{\text {pile }}=\frac{G}{2 \pi r_{\mathrm{o}}}\left[S_{\omega 1}+i S_{\omega 2}\right] w_{\text {pile }}
$$

The terms $S_{\omega 1}$ and $S_{\omega 2}$ are functions of the dimensionless frequency $a_{0}=\omega r_{0} / V_{s}$, where $\omega$ is the angular frequency of the pile motion (Fig. 4.6).

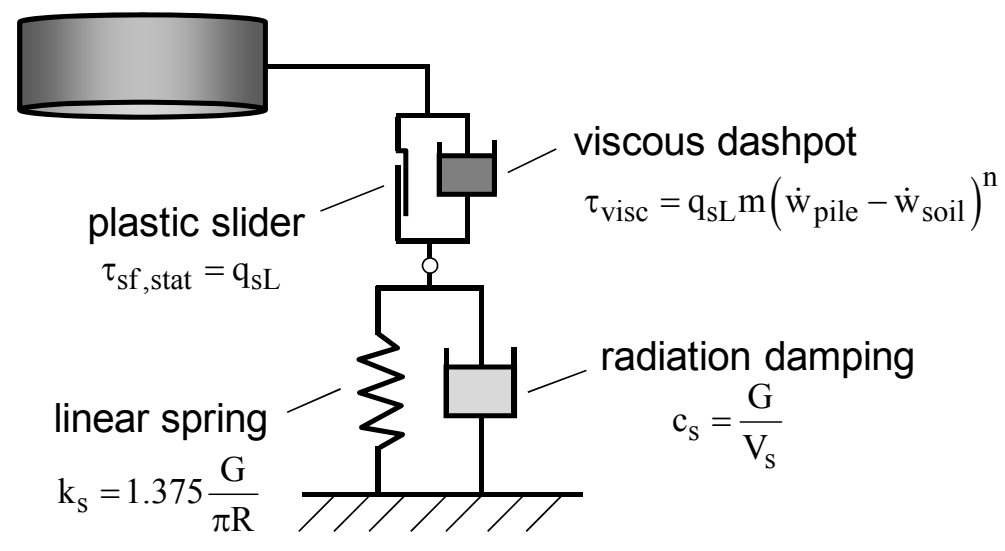

Figure 4.5. Shaft reaction model according to Randolph and Simons (1986).

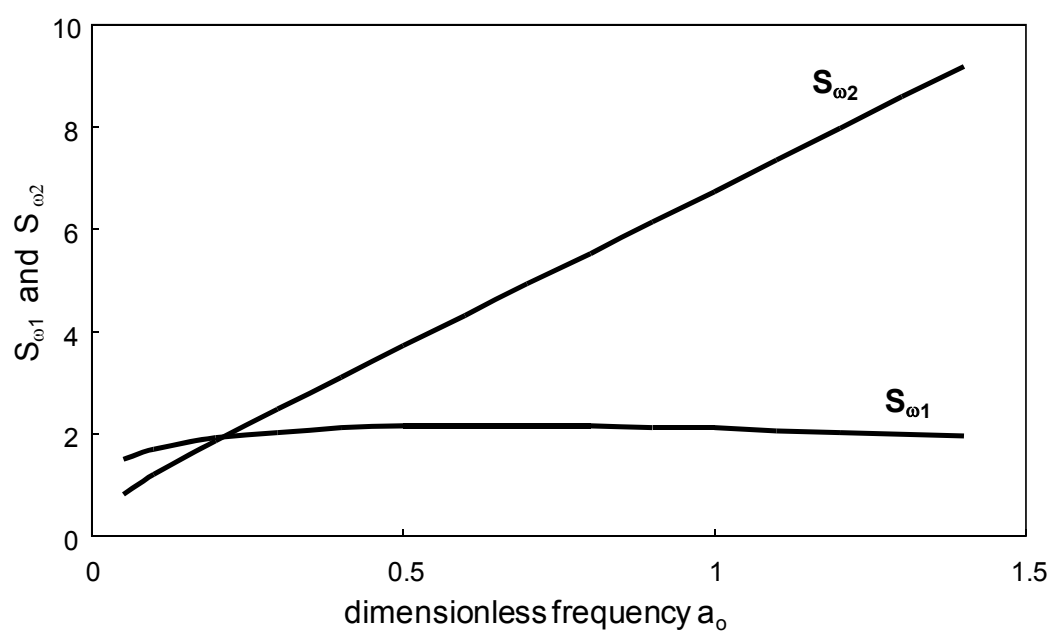

Figure 4.6. The variation of $S_{\omega 1}$ and $S_{\omega 2}$ with dimensionless frequency $a_{0}$. 
Simons and Randolph (1985) observed that, at high frequencies, $S_{\omega 1}$ and $S_{\omega 22}$ can be well approximated by 2.75 and $2 \pi \mathrm{a}_{0}$, respectively. Thus, they proposed that the spring and radiation dashpot constants of the shaft reaction model be expressed as

$$
\begin{aligned}
& k_{\mathrm{S}}=1.375 \frac{G}{\pi r_{\mathrm{O}}} \\
& c_{\mathrm{S}}=\frac{G}{V_{\mathrm{S}}}
\end{aligned}
$$

As discussed for Lysmer's model, the correct use of equation 4.13 requires $G=G_{\max }$. The model part representing the shear band takes into account the rate effect by setting the shear strength as the sum of two terms: the static resistance and the strength gain due to rate effects (viscosity):

$$
\tau_{\text {sf }}=\tau_{\text {sf,stat }}+\tau_{\text {visc }}=q_{\mathrm{sL}}+q_{\mathrm{sL}} m\left(\dot{w}_{\text {pile }}-\dot{w}_{\text {soil }}\right)^{n}
$$

where $m$ and $n$ are input parameters that control the viscous term. According to Randolph (2003), $n$ is generally in the 0.2 to 0.5 range, while $m$ takes values from 0.3 to 0.5 for sands and 2.0 to 3.0 for clays (provided that velocity is in units of $\mathrm{m} / \mathrm{s}$ ). As long as the sum of the stresses due to the linear spring and radiation dashpot do not exceed $\tau_{\mathrm{s} f \text { stat, }}$, the soil and pile move together. If $\tau_{\mathrm{s} f \text { stat }}$ gets exceeded, then slippage occurs and the soil on the outer boundary of the shear band moves differently from the pile. The strength gain due to viscosity is a function of the relative velocity between soil and pile. During slippage, the behavior is controlled by the slider and the viscosity dashpot. This is consistent with the mechanics of shaft resistance described in Section 4.2. The shaft reaction model by Randolph and Simons (1986) has gained recognition in recent years because it uses input parameters that have physical meaning and adheres to the true mechanics of the problem. One of the limitations of the model is that it does not take into account soil nonlinearity and hysteretic damping. Additionally, the spring and radiation dashpot coefficients are valid for steady state, constant-amplitude motion.

Lee et al. (1988) proposed a model that is virtually identical to that by Randolph and Simons (1986) with the only difference being that slippage happens when the spring 
reaction becomes larger than $\tau_{\text {sf,stat, }}$ at which point the radiation dashpot is deactivated. This approach is not as consistent with the mechanics of the problem and results in a discontinuity in the time history of the total reaction when the radiation dashpot is deactivated.

\subsubsection{Shaft reaction model of Holeyman (1985)}

Holeyman (1985) proposed the reaction model shown in Fig. 4.7. In consists of a spring, a viscosity dashpot and a radiation dashpot, all connected in parallel. Slippage initiates once the sum of reactions provided by these elements exceed the slider strength $\tau_{\mathrm{sf}}$. The reaction component due to the spring and viscosity is expressed as follows:

$$
\tau_{\text {spring }+ \text { visc }}=\frac{G_{\text {max }}}{R} \cdot \frac{w_{\text {pile }}}{\ln \left(R_{\mathrm{m}} / R\right)}\left[1+J\left(\dot{w}_{\text {pile }}\right)^{N}\right]
$$

assuming linear stress-strain relation and

$$
\left.\tau_{\mathrm{s}}=\frac{G_{\mathrm{max}}}{R} \cdot \frac{w_{\text {pile }}}{\ln \left(\frac{R_{\mathrm{m}} / R-\tau_{\mathrm{s}} / \tau_{\mathrm{sf}}}{1-\tau_{\mathrm{s}} / \tau_{\mathrm{sf}}}\right.}\right)\left[1+J\left(\dot{w}_{\text {pile }}\right)^{N}\right]
$$

assuming the nonlinear stress-strain relation of Kondner (1963).

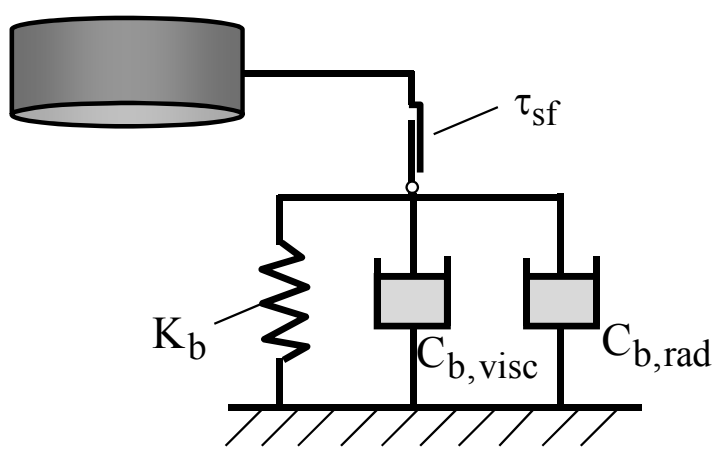

Figure 4.7. Shaft reaction model by Holeyman (1985). 
$J$ and $N$ are input parameters similar to the $m$ and $n$ used in Randolph and Simons (1986), and $R_{m}$ is the 'magical' radius proposed by Randolph and Wroth (1978). In the nonlinear case, the stiffness of the spring maintains its initial (maximum value) during unloading, introducing hysteretic damping into the formulation.

Eq. (4.15a) is based on a closed-form solution for a soil disk loaded statically. The solution is singular for a disk with an infinite lateral extent. To remove the singularity, Randolph and Wroth (1978) considered a soil disk with a finite radius $R_{\mathrm{m}}$, which is given by the following equation:

$$
R_{\mathrm{m}}=2 L(1-v)
$$

where $L$ is the pile length. Eq. (4.16) was established by matching the disk solution with results from 2-D axisymmetric finite element analysis. The reaction component due to radiation damping is expressed as

$$
\tau_{\text {rad }}=\frac{G}{V_{\mathrm{s}}} \dot{w}_{\text {pile }}
$$

which is the same as the radiation damping used by Simons and Randolph (1985). The slider strength depends on the rate of loading, as in Randolph and Simons (1986):

$$
\tau_{\text {sf }}=q_{\mathrm{sL}}\left(1+J\left(\dot{w}_{\text {pile }}-\dot{w}_{\text {soil }}\right)^{N}\right)
$$

The value of 0.2 was proposed for the parameter $N$. The Holeyman (1985) model differs from that by Randolph and Simons (1986) in two points: 1) the soil viscosity is considered active also before sliding, 2) the spring constant is valid only for static conditions, which leads to values of system's stiffness that are too small. The reason a static solution was used for the stiffness was that it can easily take into account the soil nonlinearity by the implementation of a hyperbolic stress-strain law. Having the spring stiffness equal to the small-strain stiffness during unloading constitutes a rough representation of the soil nonlinearity and hysteresis. 


\subsubsection{Shaft and base reaction models by Nguyen et al. (1988)}

Nguyen et al. (1988) proposed the model shown in Fig. 4.8. The connectivity is the same for both shaft and base. It resembles that of the Holeyman (1985) model, with the difference that the slider is not connected in series with the spring and dashpot system; it is connected in series only to the spring.

For the shaft, the spring coefficient $k_{\mathrm{s}}$ and the radiation damping coefficient $c_{\mathrm{s} \text {,rad }}$ are given by the same equations as those in the Randolph and Simons (1986) model (eqs. 4.12 and 4.13, respectively). The other dashpot represents mainly hysteretic damping, with coefficient calculated using

$$
c_{\mathrm{sH}}=\xi \frac{G}{V_{\mathrm{s}}}=\xi c_{\mathrm{s}, \mathrm{rad}}
$$

where $\xi$ is the damping ratio and $\tau_{\mathrm{sf}}$ is equal to the static limit shaft resistance. According to Nguyen et al. (1988), the effect of soil viscosity can be added to the model by increasing the value of $\xi$.

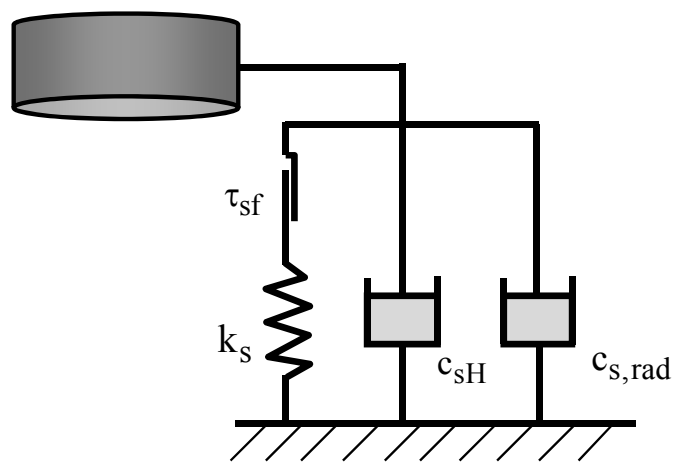

(a)

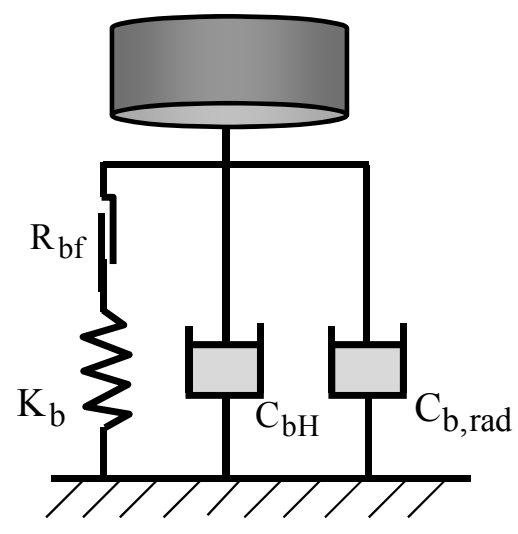

(b)

Figure 4.8. Nguyen et al. (1988) reaction models: (a) for pile shaft and (b) for pile base.

The spring radiation dashpot coefficients for the base model are the same as in Lysmer's analog (eq. 4.9 and 4.10). The coefficient for the dashpot representing hysteretic damping is 


$$
C_{\mathrm{bH}}=4 R \xi \sqrt{\frac{\pi G L R \rho_{\mathrm{p}}}{1-v}}
$$

where $\rho_{\mathrm{p}}$ is the density of the pile material. $R_{\mathrm{bf}}$ is equal to the static limit base capacity.

According to Nguyen et al. (1988), once the spring reaction component exceeds the slider strength and slippage is initiated, the radiation dashpot needs to disjoin. However, the disjoining of that dashpot results in abrupt decrease (discontinuity) in the reaction time history. Consistency with the physics of the shaft problem dictates that the slider should be placed in series with and outside the spring-radiation dashpot system (as in Holeyman 1985 and Randolph and Simons 1986), not in series only with the spring. Nguyen et al. (1988) suggested that soil nonlinearity can be accounted for by considering a degraded (secant) shear modulus based on the level of strain involved in the problem instead of $G_{\max }$.

\subsubsection{Base model by Deeks and Randolph (1995)}

Deeks and Randolph (1995) performed finite element analysis in order to validate the base reaction model based on Lysmer's analog. By matching the finite element results with several rheological model configurations, they found that the most accurate reaction model is the one shown in Fig. 4.9 (denoted as Revised A in their paper). The component connectivity of parts 1 and 2 resembles that of the Randolph and Simons (1986) shaft model. The Deeks and Randolph (1995) model contains also two masses, $m_{0}$ and $m_{1}$, which can be seen a representative of the inertia of the soil mass in the failure mechanism. Mass $m_{1}$ is connected to the system through a dashpot (part 3). The spring and dashpot coefficients are given by:

$$
\begin{aligned}
K_{0} & =\frac{4 G R}{1-v} \\
C_{0} & =\frac{4 R^{2}}{1-v} \sqrt{\rho G} \beta_{0} \\
C_{1} & =\frac{4 R^{2}}{1-v} \sqrt{\rho G} \beta_{1} \\
m_{0} & =\frac{4 R^{3} \rho}{1-v} \alpha_{0}
\end{aligned}
$$




$$
m_{1}=\frac{4 R^{3} \rho}{1-v} \alpha_{1}
$$

where $\alpha_{0}, \alpha_{1}, \beta_{0}$, and $\beta_{1}$ are model input parameters whose values depend on the soil's Poisson's ratio. The spring stiffness is the same as in the Lysmer's analog (eq. 4.9)). The parameter $\beta_{0}$ takes values in the 0.75 to 0.87 range, rendering $C_{0}$ smaller (by no more than $12 \%$ ) than the radiation damping coefficient of Lysmer's analog (eq. 4.10). Unlike $\beta_{0}$, the parameter $\beta_{1}$ shows a strong dependence on Poisson's ratio, ranging from 0.3 for $\nu=0$ to 0.83 for $v=0.45$. The finite element results showed that the masses $m_{0}$ and $m_{1}$ need never coexist. In the case of $v=0.5$ (incompressible soil, such as saturated clay), the mass $m_{1}$ vanishes while $m_{0}>0$; the opposite is true for $v<0.5$. The values of $\alpha_{0}$ and $\alpha_{1}$ range from 0.16 to 0.25 .

Once perfectly plastic action starts, radiation damping $\left(C_{0}\right)$ ceases to contribute to the soil reaction. In contrast, the dashpot connecting mass $m_{1}$ remains active even after limit base capacity is reached. The base capacity depends on the rate of loading. The viscous term of the base capacity can be expressed as

$$
R_{\mathrm{bf}, \mathrm{visc}}=R_{\mathrm{bf}, \mathrm{st}} m\left(\dot{w}_{\text {pile }}-\dot{w}_{\mathrm{soil}}\right)^{n}
$$

where $R_{\mathrm{bf}, \mathrm{st}}$ is the static base capacity, and $m$ and $n$ are the soil viscosity related parameters used in the shaft model by Randolph and Simons (1986). 


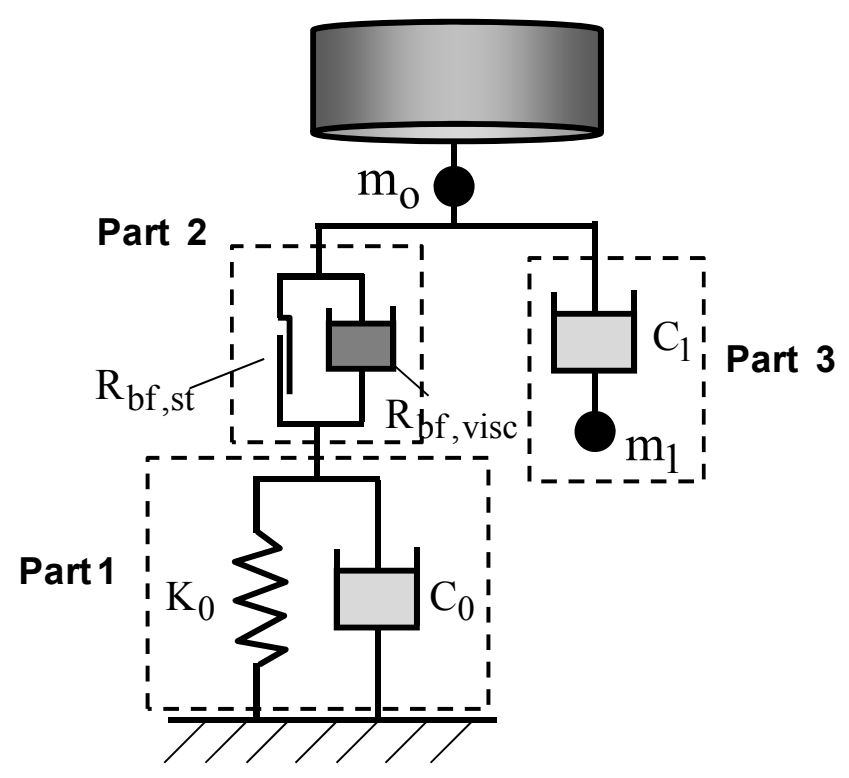

Figure 4.9. Base reaction model developed by Deeks and Randolph (1995).

Deeks and Randolph (1995) found that Lysmer's analog performs adequately in the case of mass impacting an elastic half-space, especially when $k 1 / 3$. The work of Deeks and Randolph (1995) targeted improving the commonly used Lysmer's analog so that it performs well in the case of the formation of a plastic mechanism but soil nonlinearity, hysteresis and embedment effects on stiffness and radiation damping (resulting from the fact that the tip of pile is usually at a depth many times its width, and not on the surface of a half-space) remained unaddressed.

\subsubsection{Shaft model by El Naggar and Novak (1994)}

El Naggar and Novak (1994) improved the Randolph and Simons (1986) model by introducing the effects of soil nonlinearity and hysteresis. They considered three distinct zones around the pile shaft: 1) thin shear band in contact with the shaft wall, 2) an inner zone where soil nonlinearity and hysteretic damping dominates, and 3) an outer zone where the soil behaves linearly (Fig. 4.10a). The model part corresponding to the shear band and the outer field (Fig. 4.10b) are the same as in the shaft resistance model of Randolph and Simons (1986).

The shear stress in the inner zone follows the nonlinear stress-strain relationship of Kondner (1963): 


$$
\tau_{\text {inner }}=\frac{G_{\text {max }}}{r_{\mathrm{o}}} \frac{w_{\text {pile }}}{\ln \left(\frac{r_{1} / r_{\mathrm{o}}-\tau_{\text {inner }} / \tau_{\text {sf }}}{1-\tau_{\text {inner }} / \tau_{\text {sf }}}\right)}+c_{\mathrm{m}} \dot{w}_{\text {pile }}
$$

where $r_{0}=R$ (the pile radius), $r_{1}$ is the outer radius of the inner zone. The radius $r_{1}$ can be taken as $1.1 r_{0}$. During unloading, the spring of the inner zone model part is assumed to behave elastically, having a constant value equal to

$$
k_{\mathrm{n} 1, \max }=\frac{G_{\max }}{r_{\mathrm{o}}} \frac{1}{\ln \left(r_{1} / r_{\mathrm{o}}\right)}
$$

Unloading with the maximum spring modulus introduces some degree of hysteretic damping; $c_{\mathrm{m}}$ is an extra damping factor which may express additional hysteretic damping and/or soil viscosity (no guidelines are given in El Naggar and Novak (1994) regarding its values). The mass of the inner zone is lumped into two point masses located at the boundaries of the inner zone (Fig. 4.10b).

The model shares all the advantages of the Randolph and Simons (1986) model and enhances it with soil nonlinearity and hysteretic damping. The derivation of the equations for the inner zone are based on the static solution of Randolph and Wroth (1978); for these equations to be valid for the dynamic problem of pile driving, the wavelength of the waves traveling radially in the soil must be much larger (more than 4 times) than the extent of the inner zone. As in the Randolph and Simons (1986) model, the spring and dashpot coefficients for the outer field are valid for a harmonic steady-state pile response. The spring and dashpot coefficients are set to be functions of the pile radius $r_{0}$ instead of the radius $r_{1}$ of the boundary between inner and outer fields. Since the outer-field springs and dashpots are acting on that boundary and not directly on the pile shaft, representation in terms of $r_{1}$ would probably be more appropriate. 


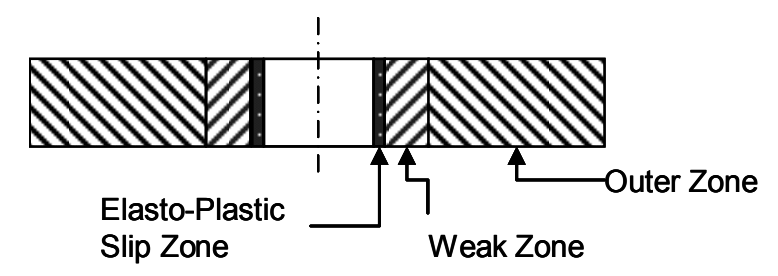

(Section View)

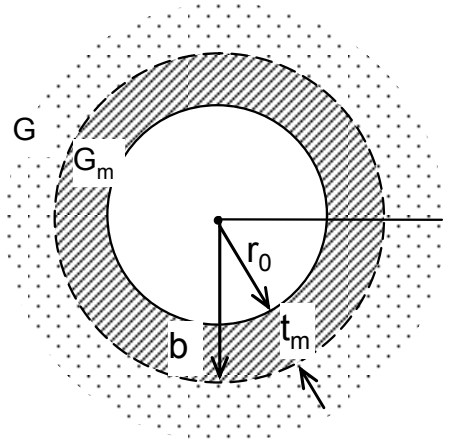

(Plan View)

(a)

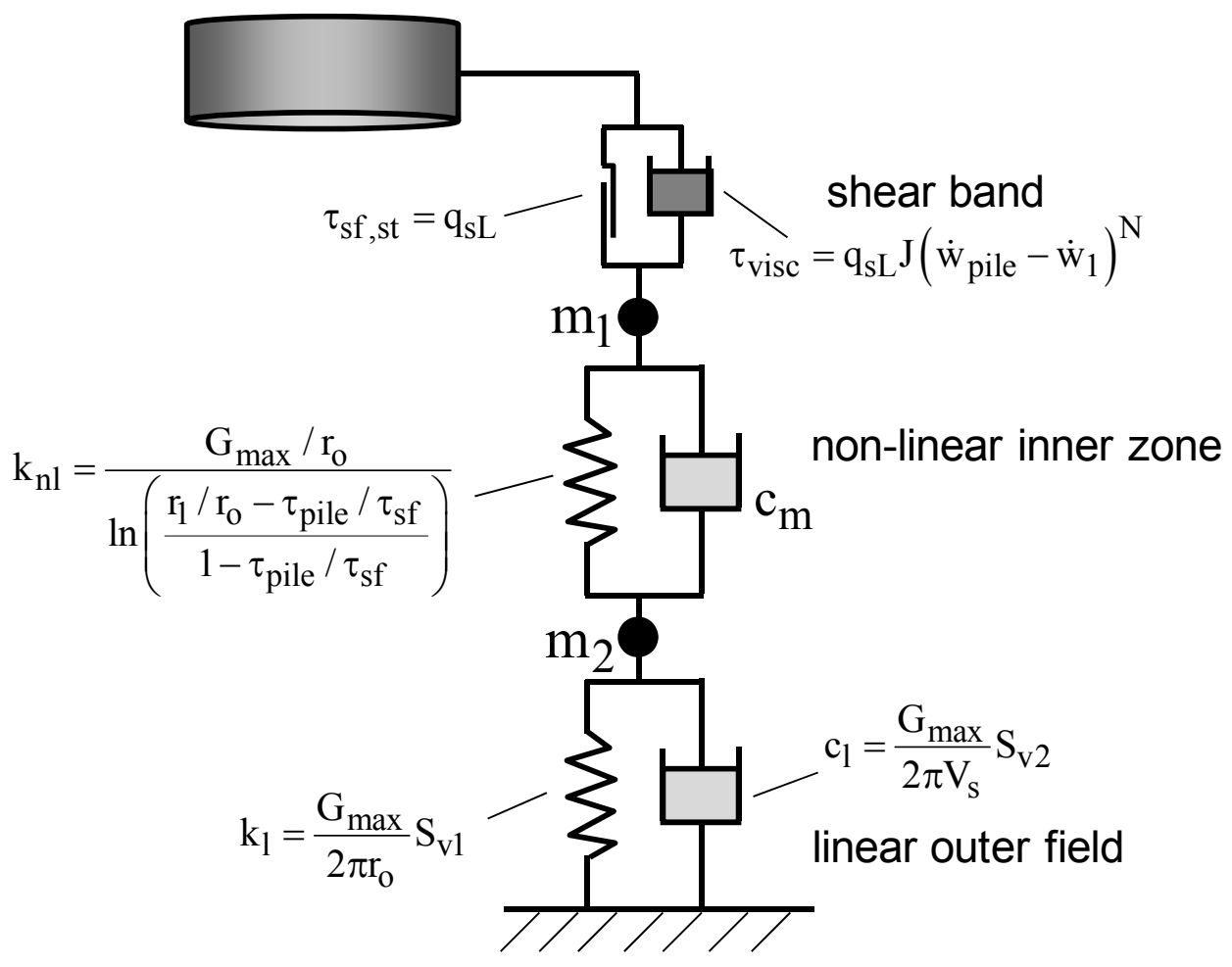

(b)

Figure 4.10. Shaft reaction model proposed by El Naggar and Novak (1994). 


\subsubsection{Shaft model by Michaelides et al. (1998)}

Michaelides et al. $(1997,1998)$ proposed a shaft reaction model that accounts for soil nonlinearity and hysteretic damping for use in the field of foundation vibrations. Their solution is an extension of the Novak et al. (1978) solution, which is derived by solving the wave equation of vertical shear waves propagating inside a thin soil disk (1-D axisymmetric conditions) with the pile in its center:

$$
\frac{\partial \tau}{\partial r}+\frac{\tau}{r}=\rho \frac{\partial^{2} w}{\partial t^{2}} \Rightarrow G\left(\frac{d^{2} w}{d r^{2}}\right)+\left(\frac{d G}{d r}+\frac{G}{r}\right) \frac{d w}{d r}=\rho \frac{d^{2} w}{d t^{2}}
$$

If the shear modulus is assumed to be constant, the solution of eq. (4.29) produces the spring and dashpot coefficients of Novak et al. (1978) given by eq. (4.11). Michaelides et al. (1998) solved eq. (4.29) assuming that the secant shear modulus degrades and the hysteretic damping ratio increases with cyclic shear strain following Ishibashi and Zang (1993). Since the cyclic shear strain decreases with radial distance from the pile wall, the secant shear modulus and hysteretic damping become functions of the radial distance (Fig. 4.11).

The solution of the differential equation (4.29) results in the following spring stiffness and radiation dashpot coefficients:

$$
\begin{gathered}
k_{\mathrm{s}}=\left\{\frac{1.8 G_{\max }}{2 \pi R}\left(1+0.5 \sqrt{\frac{\omega R}{\mathrm{~V}_{\mathrm{s}}}}\right)\right\} \frac{1-\frac{0.6 \Lambda}{1-\Lambda}\left(\omega R / \mathrm{V}_{\mathrm{s}}\right)^{1.5}}{1-1.2 \Lambda} \\
C=\left[\frac{1.2 \rho V_{\mathrm{s}}}{\left(\omega R / V_{\mathrm{s}}\right)^{0.25}}+\frac{\xi_{\text {min }} 1.8 G_{\max }\left(1+0.5 \sqrt{\frac{\omega R}{\mathrm{~V}_{\mathrm{s}}}}\right)}{\omega \pi R}\right]\left(1-0.84 \Lambda\left(1+0.66 \log \sqrt{\frac{\omega R}{V_{\mathrm{s}}}}\right)\right)
\end{gathered}
$$




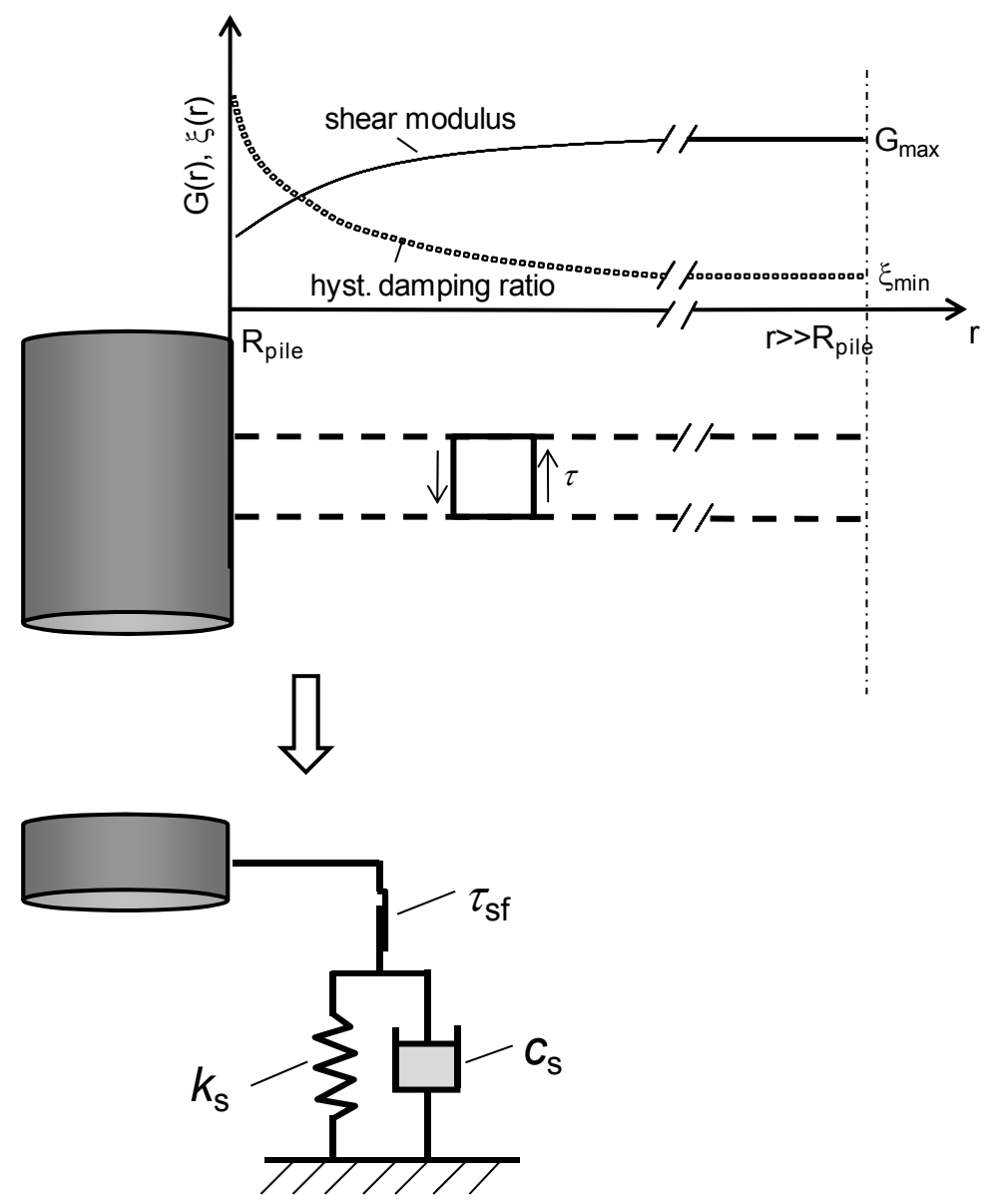

Figure 4.11. Shaft reaction model proposed by Michaelides et al. $(1997,1998)$.

The parameter $\Lambda$ expresses the effect of shear stress amplitude on soil stiffness and damping and is given by

$$
\Lambda=600 \frac{\tau_{\mathrm{s,max}}}{G_{\max }} \exp \left[-1.39 \frac{P I}{125}\right]
$$

where $\tau_{\mathrm{s}, \max }$ is the maximum shear strength observed at the pile-soil interface, and $\mathrm{Pl}$ is the soil plasticity index. The first term inside the brackets in eq. (4.31) represents the radiation damping, while the second term represents the hysteretic damping. The minimum (smallstrain) damping ratio $\xi_{\min }$ is usually found to be in the $0.5 \%$ to $1 \%$ range. The shear stress amplitude $\tau_{\mathrm{s}, \max }$ needed for the calculation of the nonlinearity parameter and the angular 
frequency $\omega$ are not known a priori. Therefore, use of the Michaelides et al. (1998) model requires the pile analysis to be performed twice. The first (trial) analysis will use initial

guesses for the values of $\tau_{\mathrm{s}, \max }$ and $\omega$. The second (final) analysis will use the $\tau_{\mathrm{s}, \max }$ and $\omega$ produced by the trial analysis. Further iterations are not needed since the frequency of motion and the shear stress amplitude are not sensitive to the parameters of the shaft reaction model in the case of the pile driving problem. The angular frequency is mainly controlled by the mechanical properties of the pile, and the shear stress amplitude often equals the limit shaft resistance because of pile sliding.

Although Michaelides et al. (1998) considered that the slider strength $\tau_{\mathrm{sf}}$ is independent of the velocity of the pile motion, it can be made a function of the velocity following eq. (4.14). The Michaelides et al. $(1997,1998)$ model constitutes a rigorous approach (subject to 1-D limitations) satisfying the physics and mechanics involved in the development of soil reaction on the pile shaft provided that the pile oscillates harmonically with constant amplitude (steady-state). This constitutes a limitation of the model when considering the highly transient nature of the pile motion during driving (impact pulse).

\subsubsection{Continuum shaft and base models by Holeyman}

Holeyman developed shaft and base models that are based on a continuum approach. This means that part of the soil mass surrounding the pile is included in the pile driving analysis as a continuum instead of a system of springs, dashpots and sliders. The main advantage of this approach are: 1) any stress-strain relationship can be considered in the solution of the problem, thus allowing capturing soil nonlinearity and hysteretic damping realistically and 2) a solution valid for the highly transient problem of pile driving can be achieved without relying on equations derived for harmonic steady-state oscillations. Their main disadvantage is that they increase significantly the computational cost. Nonetheless, the computational cost is still much less than that of finite element simulations.

Holeyman (1988) proposed the base reaction shown in Fig. 4.12a. It assumes the soil reaction at the pile base is controlled by the region under the pile base with the shape of a truncated cone. The increase of the cone radius with depth is described by the following equation

$$
\mathrm{r}(\mathrm{z})=\mathrm{R}+\frac{1-\mathrm{v}}{\sqrt{0.85}} \mathrm{z}
$$




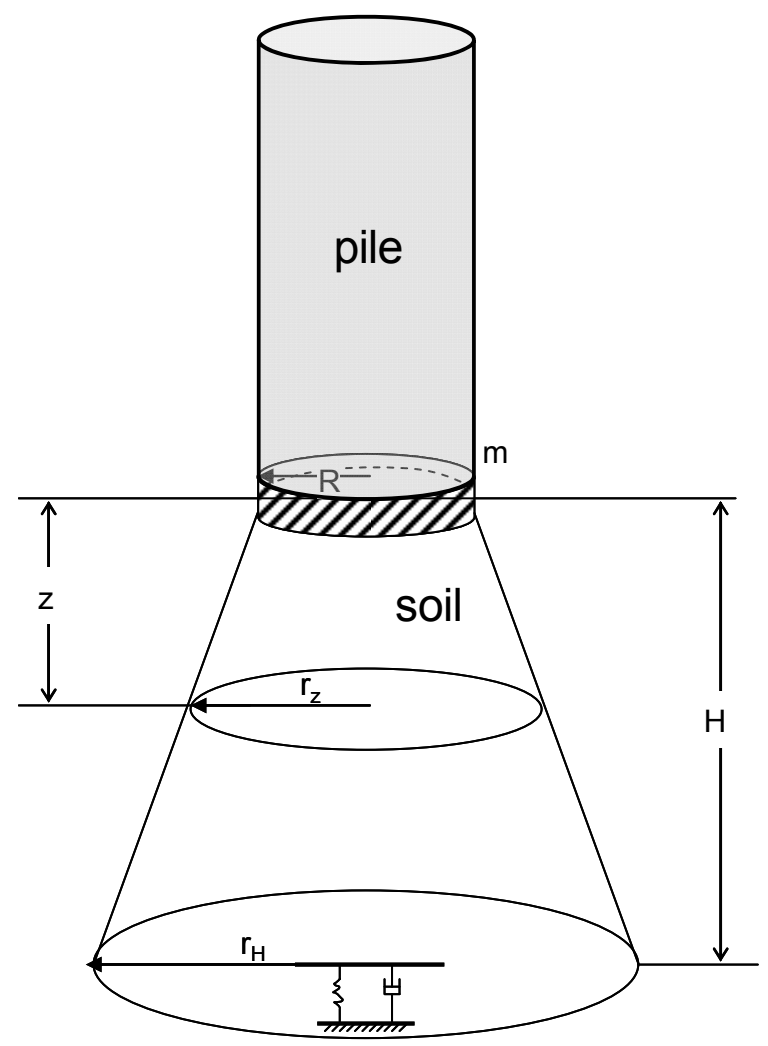

(a)

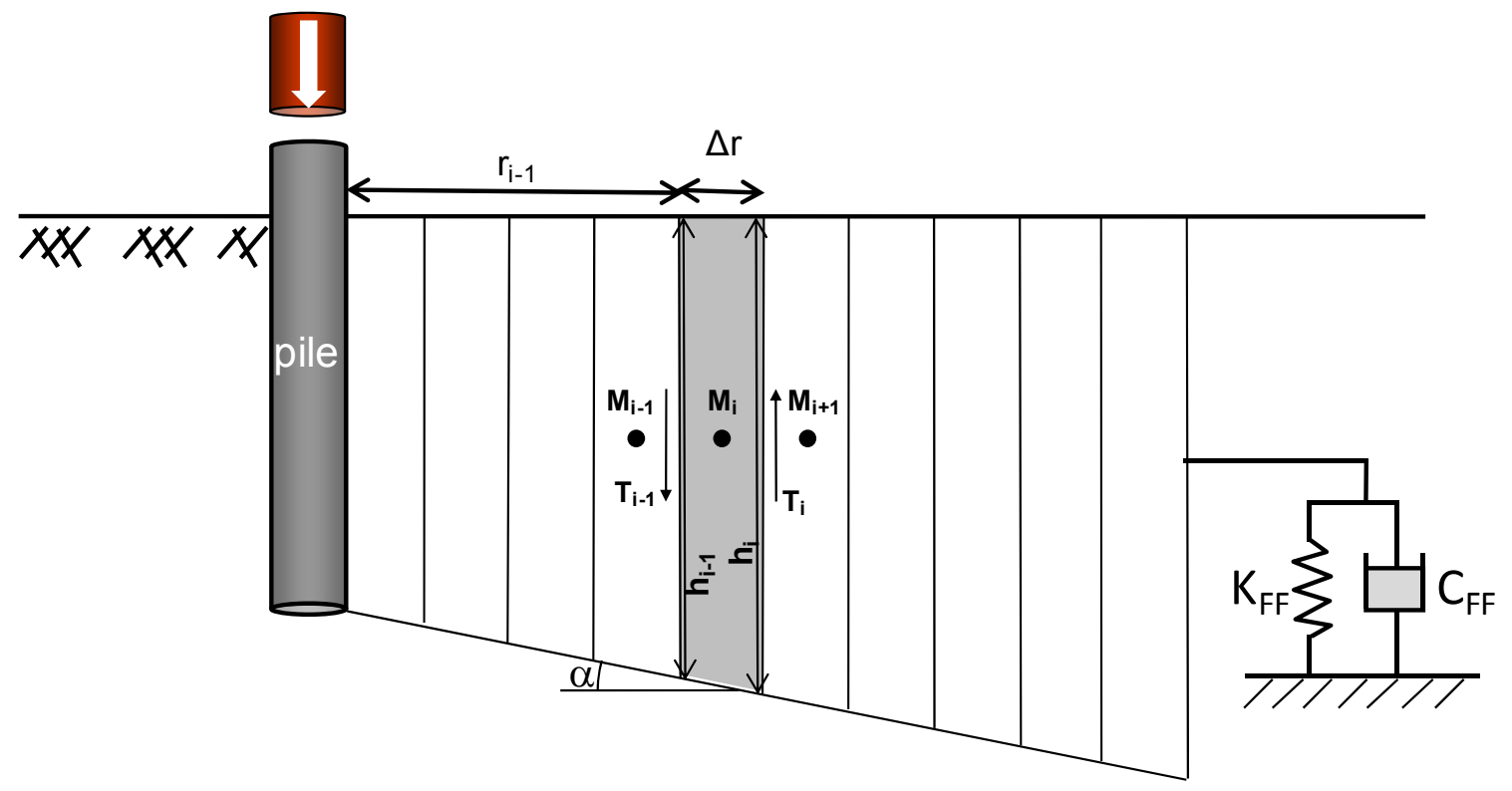

$\mathrm{M}_{\mathrm{i}} \ddot{\mathrm{w}}_{\mathrm{i}}=2 \pi \mathrm{r}_{\mathrm{i}} \mathrm{h}_{\mathrm{i}} \tau_{\mathrm{i}}-2 \pi \mathrm{r}_{\mathrm{i}-\mathrm{i}} \mathrm{h}_{\mathrm{i}-\mathrm{i}} \tau_{\mathrm{i}-1}$

(b)

Figure 4.12. Reaction models based on a continuum approach: a) base model by Holeyman (1988), b) shaft model by Holeyman et al. (1996). 
The truncated cone is discretized into lumped masses that are connected to each other through nonlinear springs. The spring coefficient is calculated from the shear modulus based on the following equation

$$
K=\frac{G}{0.85(1-v)^{2}} \pi r^{2}
$$

The degradation of $G$ follows the hyperbolic law by Kondner (1963), similarly to eq. 4.15b. The elastic half space lying below the base of the truncated cone is represented by a spring. In essence, Holeyman (1988) extended the 1-D lumped mass formulation to the region below the pile.

For the shaft case, the soil surrounding the pile shaft is discretized into a series of concentric cylinders (Fig 4.12b) with horizontal thickness $\Delta r$. The height of the innermost cylinder is equal to the pile length. The height of the cylinders increases linearly with radial distance from the shaft according to

$$
h(\mathrm{r})=L+(r-R) \alpha
$$

in order to consider the dispersion of waves in the vertical direction. The parameter $\alpha$ expresses the rate of increase, $L$ is the pile length and $R$ is the pile radius (actual or equivalent). The mass $M_{\mathrm{i}}$ of each cylinder is lumped at its center. The only mode of deformation taken into account is vertical shearing. The solution considers the dynamic equilibrium of each cylinder:

$$
\mathrm{M}_{\mathrm{i}} \ddot{\mathrm{w}}_{\mathrm{i}}=\mathrm{T}_{\mathrm{i}}-\mathrm{T}_{\mathrm{i}-1}=2 \pi \mathrm{r}_{\mathrm{i}} \mathrm{h}_{\mathrm{i}} \tau_{\mathrm{i}}-2 \pi \mathrm{r}_{\mathrm{i}-\mathrm{i}} \mathrm{h}_{\mathrm{i}-\mathrm{i}} \tau_{\mathrm{i}-1}
$$

where $\tau_{\mathrm{i}}$ and $\tau_{\mathrm{i}-1}$ are shear stresses acting on the outer and inner boundaries of the cylinder, respectively. The system of equations of motion for the soil is solved for the soil vertical displacement $w_{i}$ using explicit integration methods. A hyperbolic stress-strain relationship (such as Duncan and Chang 1970) can be used for calculating the shear stress as a function shear strain. More advanced constitutive models can also be considered without 
need to change the formulation (Vanden Berghen 2001). The soil shear strength is taken as a function of the rate of strain:

$$
\tau_{\max , \mathrm{i}}^{\mathrm{dyn}}=\tau_{\max }^{\mathrm{st}} \times\left[1+\mathrm{J}\left(\frac{\dot{\mathrm{w}}_{\mathrm{i}}-\dot{\mathrm{w}}_{\mathrm{i}-1}}{\Delta \mathrm{r}}\right)^{0.2}\right]
$$

The model is completed by assigning far-field (absorbing) boundary at the outer cylinder consisting of a spring $\left(K_{\mathrm{FF}}\right)$ and a radiation dashpot $\left(C_{\mathrm{FF}}\right)$. The equations for the spring and dashpot coefficients are based on the Novak et al. (1978) solution:

$$
\begin{aligned}
& k_{\mathrm{FF}}=1.375 \frac{G_{\mathrm{max}}}{\pi r_{\mathrm{o}}} \\
& c_{\mathrm{FF}}=\frac{G_{\mathrm{max}}}{V_{\mathrm{s}}}
\end{aligned}
$$

Holeyman et al. (1996) use the pile radius $R$ for $r_{0}$, although the radius of the outer boundary is much larger than that of the pile, resulting in a conceptual inconsistency.

One limitation of the proposed continuum models is that they do not distinguish between the internal soil shear strength and the resistance on the foundation element (limit shaft resistance and pile base bearing capacity). In reality, the shaft resistance is expected to be smaller than $\tau_{\max }$. A major drawback of the shaft model is that the pile is assumed to be rigid and that the soil is homogenous. There is only one set of concentric cylinders extending through the full pile height. In order to be used in problems where the soil profile consists of more than one layer, average soil properties need to be assigned. Assuming a homogenous soil profile, the base model is less rigorous than the shaft model because of the complexities involved in the dynamic response of the base, although both base and shaft models follow the same concept. The response of the base is a 2-dimensional axisymmetric problem due to the limited lateral extent of the base compared to the soil medium. A more rigorous solution would need to address the base problem as a 2-D problem. In contrast, the shaft resistance problem can be well approximated by a 1-D approach. 


\subsection{Final remarks}

A comparison between the models presented in this Chapter is summarized in Tables 4.1 and 4.2. The original Smith (1960) model with the set of subsequently updated quake and damping parameters is today the state-of-practice in the field of pile driving analysis. It is in use in the most popular pile driving analysis software, such as CAPWAP and GRLWEAP. The previous sections demonstrated that significant efforts have been made to develop improved reaction models. The improved models demonstrate clearly that the spring and dashpot coefficients are not proportional to the limit resistance, as in Smith's model, but depend on the soil stiffness, soil density and the pile radius.

Significant improvements have been made in the case of shaft reaction models. The most notable among them with respect to analytical rigor are those by Randolph and Simons (1986) and Michaelides et al. (1998). The model by Michaelides et al. (1998) is probably the most advanced and complete rheological model for the shaft resistance. However, these models are based on steady-state harmonic solutions, which are in contrast with the highly transient nature of the pile response during driving. Continuum models, especially in the case of shaft reaction, are advantageous because they allow the use of any nonlinear (or plasticity) models without the complications of 1-D analysis formulations and are applicable to any type of dynamic loading.

Nearly all the improved base reaction models employ the Lysmer's analog in some form or another. Compared to the shaft models, little progress has been made for base models in terms of analytical rigor because of the complex mechanics involved in the 2-D problem of pile base response. Lysmer's analogue model assumes that the impacting mass is on the surface of a half-space. Any embedment effects on stiffness and radiation damping are ignored. Given the very high embedment ratio (penetration depth/pile width) of piles, it is expected that Lysmer's analog's stiffness and radiation damping will be smaller than those observed in the pile driving problem. 
Table 4.1. Summary of shaft reaction model

\begin{tabular}{|c|c|c|c|c|c|c|c|c|c|}
\hline Model & Type & $\begin{array}{l}\text { consistency } \\
\text { with actual } \\
\text { mechanics }\end{array}$ & $\begin{array}{l}\text { Input parameters } \\
\text { based on soil } \\
\text { properties }\end{array}$ & $\begin{array}{l}\text { radiation } \\
\text { damping }\end{array}$ & $\begin{array}{c}\text { soil } \\
\text { nonlineartiy }\end{array}$ & $\begin{array}{l}\text { hysteretic } \\
\text { damping }\end{array}$ & $\begin{array}{l}\text { rate effect } \\
\text { on strength }\end{array}$ & $\begin{array}{l}\text { highly } \\
\text { transient } \\
\text { (impact) } \\
\text { motion }\end{array}$ & Comments/Limitations \\
\hline Smith (1960) & shaft & & & & & & & & $\begin{array}{l}\text { All damping effects (radiation, } \\
\text { hysteresis, viscosity) are merged } \\
\text { into one term with fully empirical } \\
\text { constants }\end{array}$ \\
\hline $\begin{array}{c}\text { Randolph and } \\
\text { Simons }(1985,1986)\end{array}$ & shaft & & & & & & & & \\
\hline Holeyman (1985) & shaft & & & & & & & & $\begin{array}{l}\text { Approximate treatment of soil } \\
\text { nonlinearity and hysteretic } \\
\text { damping. Nonlinear formulation } \\
\text { based on static solution }\end{array}$ \\
\hline Nguyen et al. (1988) & shaft & & & $\checkmark$ & & & & & \\
\hline $\begin{array}{l}\text { El Naggar and } \\
\text { Novak (1994) }\end{array}$ & shaft & & & & & & & & $\begin{array}{l}\text { Approximate treatment of soil } \\
\text { nonlinearity and hysteretic } \\
\text { damping. Nonlinear formulation } \\
\text { based on static solution }\end{array}$ \\
\hline $\begin{array}{l}\text { Michaelides et al. } \\
\text { (1998) }\end{array}$ & shaft & & & & & & & & \\
\hline $\begin{array}{l}\text { Holeyman et al. } \\
\text { (1996) }\end{array}$ & shaft & & & & & & & & $\begin{array}{l}\text { No distinction between soil shear } \\
\text { strength and limit shaft resistance. } \\
\text { Far-field boundary condition } \\
\text { depends on the pile radius instead } \\
\text { of the boundary radius. Only a } \\
\text { single soil layer around the pile. }\end{array}$ \\
\hline
\end{tabular}


Table 4.2. Summary of base reaction model

\begin{tabular}{|c|c|c|c|c|c|c|c|c|c|c|}
\hline Model & Type & $\begin{array}{l}\text { consistency } \\
\text { with actual } \\
\text { mechanics }\end{array}$ & $\begin{array}{l}\text { Input } \\
\text { parameters } \\
\text { based on soil } \\
\text { properties }\end{array}$ & $\begin{array}{l}\text { radiation } \\
\text { damping }\end{array}$ & $\begin{array}{c}\text { soil } \\
\text { nonlineartiy }\end{array}$ & $\begin{array}{l}\text { hysteretic } \\
\text { damping }\end{array}$ & $\begin{array}{l}\text { rate effect } \\
\text { on strength }\end{array}$ & $\begin{array}{l}\text { highly } \\
\text { transient } \\
\text { (impact) } \\
\text { motion }\end{array}$ & $\begin{array}{l}\text { effect of } \\
\text { embedment } \\
\text { on stiffness } \\
\text { and damping }\end{array}$ & Comments/Limitations \\
\hline Smith (1960) & base & & & & & & & & & $\begin{array}{l}\text { All damping effects } \\
\text { (radiaton, hysteresis, } \\
\text { viscosity) are merged } \\
\text { into one term with fully } \\
\text { empirical constants }\end{array}$ \\
\hline $\begin{array}{c}\text { Based on } \\
\text { Lysmer's analogue }\end{array}$ & base & & & & & & & & & \\
\hline $\begin{array}{l}\text { Nguyen et al. } \\
\text { (1988) }\end{array}$ & base & & & & & & & & & \\
\hline Holeyman (1988) & base & & $v$ & $\boldsymbol{v}$ & $\checkmark$ & $\checkmark$ & $V$ & $\boldsymbol{v}$ & & \\
\hline $\begin{array}{c}\text { Deeks and } \\
\text { Randolph (1995) }\end{array}$ & base & & & & & & & & & \\
\hline
\end{tabular}




\section{CHAPTER 5. PROPOSED SOIL REACTION MODEL FOR PILE DRIVING ANALYSIS}

\subsection{Introduction}

In the previous chapters, we saw that significant research has been done on methods for the dynamics analysis of pile driving. These can be grouped into numerical methods, such as the 1-dimensional lumped-mass approach (i.e., Smith's approach) and the finite element method, and semi-analytical methods, such as the method of characteristics. Finite element analysis is most effective in producing realistic and accurate solutions, but is too expensive computationally to use in pile engineering practice. Semi-analytical methods are mathematically elaborate but not as versatile. In contrast, the 1-D lumped-mass approach is comparatively simple, is computationally economical, and allows the implementation of virtually any soil reaction model. It is currently used in most pile driving analysis software. The type of discretization (lumped mass vs. continuous) of the pile and the algorithms used for the integration of the equation of motion (or of the wave equation) is not as critical as the soil reaction models used. The success in simulation of pile driving and reliable prediction of the pile response rests mostly on the use of accurate soil reaction models.

The soil reaction models that have been proposed so far were discussed in Chapter 4. There has been considerable progress in the course of the last three decades in the formulation of soil reaction models. More recent models distinguish between the three different types of damping (radiation, hysteresis and viscosity) and have input parameters that are either well anchored on standard soil properties or can be determined based on laboratory test data. The nonlinearity of the soil stress-strain relations has been taken into consideration.

The most complete shaft resistance models are those by Holeyman et al. (1996) and Michaelides et al. (1998). Still, each of these two models has certain limitations. The model by Michaelides et al. (1998) is based on a steady-state solution, while the pile motion during driving is highly transient (impact pulse). The Holeyman et al. (1996) model does not have this deficiency because it considers the surrounding soil as a continuum and its response is determined during the dynamic analysis; the solution will be valid for any type of pile input motion. However, the formulation of that model appears to be incomplete with respect to the behavior within the shaft shear band (as indicated by Charue 2004) and the far-field 
conditions at the outer boundary of the analysis domain. The models of base reaction are less complete. The work by Deeks and Randolph (1995) is the most thorough investigation on the rheological models for pile base. However, that model has been calibrated for predicting the response of an elastic-perfectly plastic soil; soil nonlinearlity and hysteresis are not included. The development of dynamic base resistance in the Holeyman (1988) model is a combination of a number of sub-mechanisms that are hard to decouple in the manner of the truncated cone model of Holeyman (1988). All of the base models proposed so far neglect the effect of embedment on stiffness and damping.

In this Chapter, we develop and formulate shaft and base reaction models that are grounded on the actual physics and mechanics of the pile driving problem and are as realistic as possible. The proposed models will be validated against data from pile load tests on full-scale and model (small-scale) piles in Chapter 7.

\subsection{Proposed shaft reaction model}

\subsubsection{Basic soil disk formulation for elastic soil}

The shaft reaction model by Holeyman et al. (1996) has demonstrated the advantages of the continuum approach (Table 4.1) and, thus, the proposed model will follow these lines. Considering the soil mass explicitly in the formulation of the soil reaction model has the significant advantage that the solution will be valid for any type of input motion. Moreover, any stress-strain relation can be assumed allowing a versatile treatment of soil nonlinearity and hysteresis. Our formulation will be based on the thin soil disk approach, which has been used numerous times for the simulation of the soil response around the pile shaft under static conditions (e.g., Randolph and Wroth 1978; Potts and Martins 1982, Loukidis and Salgado 2008).

Let us assume a thin soil disk with an annulus area occupied by the pile with radius $r_{0}$ (Fig. 5.1). The thin soil disk is of finite extent with outer radius $r_{\mathrm{f}}$. The dynamic equilibrium equation of a soil element at any radial distance $r$ and time $t$ can be written as

$$
\frac{\partial \tau}{\partial r}-\frac{\tau}{r}=\rho \frac{\partial^{2} w}{\partial t^{2}}
$$


where $w$ is the vertical soil displacement, $\tau$ is the shear stress, and $\rho$ is the soil density. The two terms on the left-hand side of eq. (5.1) are subtracted (instead of being added as in most publications and in eq. 4.29) because we assume that the positive direction for the displacement $w$ is downwards. Likewise, depth $z$ is positive downwards. This sign convention is more suitable for the problem of pile driving since, once struck by the ram, the pile moves mostly downwards. At this initial stage, we will also assume that the soil is elastic. This will allow us to establish appropriate far-field boundary conditions at the outer boundary of the disk. For an elastic soil, $\tau$ is always equal to $G_{\gamma}(=G \mathrm{~d} w / \mathrm{d} r)$, and eq. (5.1) can be rewritten as:

$$
G \frac{\partial^{2} w}{\partial r^{2}}-G \frac{\partial w}{\partial r}=\rho \frac{\partial^{2} w}{\partial t^{2}}
$$

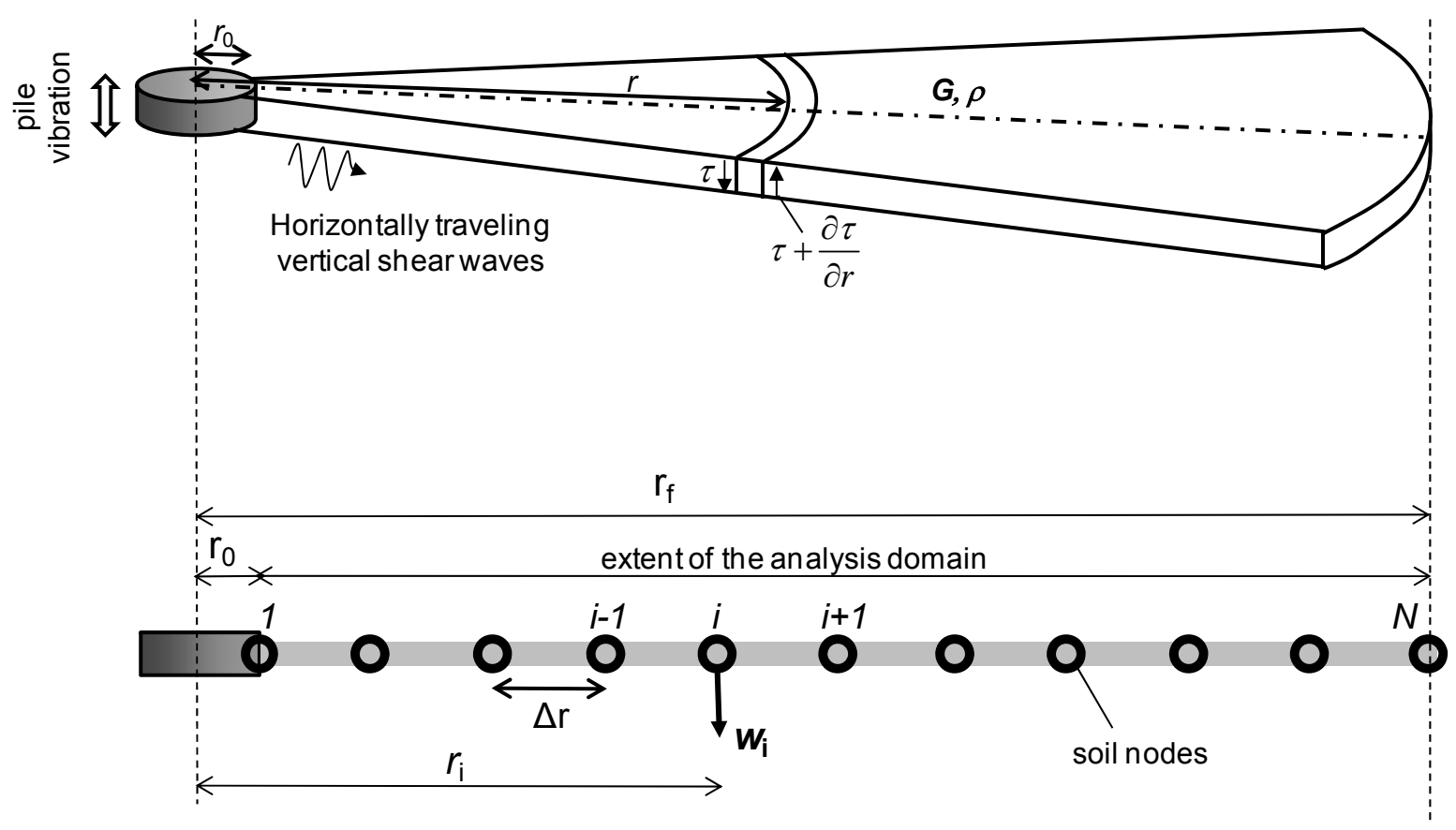

Figure 5.1. Soil disk around pile shaft and finite difference discretization.

The differential equation (5.2) can be solved analytically if we assume that the motion is a steady-state harmonic oscillation (i.e., $w=w_{0} \exp [i \omega t]$ ), as done by Novak et al. (1978). The 
specific characteristics of the vertical motion of different pile cross-sections during driving are not known a priori; they will be the product the analysis. Since our goal is to produce a formulation that can accommodate any type of motion, especially that due to an impact-type pulse propagating down the pile, we will solve eq. (5.2) numerically. For this purpose, we assume $N_{\mathrm{s}}$ number of points (soil nodes) along the radial axis from $r_{0}$ to $r_{\mathrm{f}}$ separated by radial distance $\Delta r$ (Fig. 5.1). Time is divided into small but finite increments $\Delta t$. Using the central finite difference approximation, the first and second derivatives appearing in eq. (5.2) for the node $i$ are given by the following equations:

$$
\begin{aligned}
& \frac{\partial w}{\partial r} \approx \frac{w_{i-1}^{(t)}-w_{i+1}^{(t)}}{2 \Delta r} \\
& \frac{\partial^{2} w}{\partial r^{2}} \approx \frac{w_{i-1}^{(t)}-2 w_{i}^{(t)}+w_{i+1}^{(t)}}{\Delta r^{2}} \\
& \frac{\partial^{2} w}{\partial t^{2}} \approx \frac{w_{i}^{(t+\Delta t)}-2 w_{i}^{(t)}+w_{i}^{(t-\Delta t)}}{\Delta t^{2}}
\end{aligned}
$$

The superscripts in parenthesis (i.e. $(t-\Delta t),(t),(t+\Delta t))$ denote the time that the displacement $w$ corresponds to. Therefore, eq. (5.2) can be approximated by

$$
G \frac{w_{i-1}^{(t)}-2 w_{i}^{(t)}+w_{i+1}^{(t)}}{\Delta r^{2}}-\frac{G}{r_{i}} \frac{w_{i-1}^{(t)}-w_{i+1}^{(t)}}{2 \Delta r}=\rho \frac{w_{i}^{(t+\Delta t)}-2 w_{i}^{(t)}+w_{i}^{(t-\Delta t)}}{\Delta t^{2}}
$$

Provided that we know the displacements at all the nodes at times $(t)$ and $(t-\Delta t)$, we can calculate the displacement at time $(t+\Delta t)$ at each node $i$ by solving eq. (5.3) with respect to $w_{i}^{(t+\Delta t):}$

$$
w_{i}^{(t+\Delta t)}=\frac{\Delta t^{2}}{\rho}\left[G \frac{w_{i-1}^{(t)}-2 w_{i}^{(t)}+2 w_{i+1}^{(t)}}{\Delta r^{2}}-\frac{G}{r_{i}} \frac{w_{i-1}^{(t)}-w_{i+1}^{(t)}}{2 \Delta r}\right]+2 w_{i}^{(t)}-w_{i}^{(t-\Delta t)}
$$

Before the pile starts moving, the entire soil disk is at rest; this means that the displacements at all nodes are equal to zero. Therefore, all nodal displacements will be known at the beginning of the calculations, and eq. (5.4) will be used for calculating the soil 
displacements at subsequent time increments. At each time increment, eq. (5.4) Is applied successively for all nodes, starting from the node at the inner boundary (node 1).

\subsubsection{Free-field-consistent boundary conditions}

The discretized soil disk represents only a part of the soil around the pile (the near field). The presence of the outer soil mass (far field) needs to be represented in the model formulation. The far-field soil absorbs fully the waves traveling radially in the soil disk. If no absorbing boundary conditions are provided to the model, then the energy emitted from the pile shaft will be fully reflected at the outer boundary of the analysis domain. In numerical analysis (finite difference method or finite element method), absorbing boundaries are introduced through radiation dashpots with coefficient values such that any wave traveling outwards vanishes after reaching that boundary (Fig 5.2). Use of an inconsistent dashpot coefficient is easily noticeable, since they will generate reflection waves that will move towards the pile.
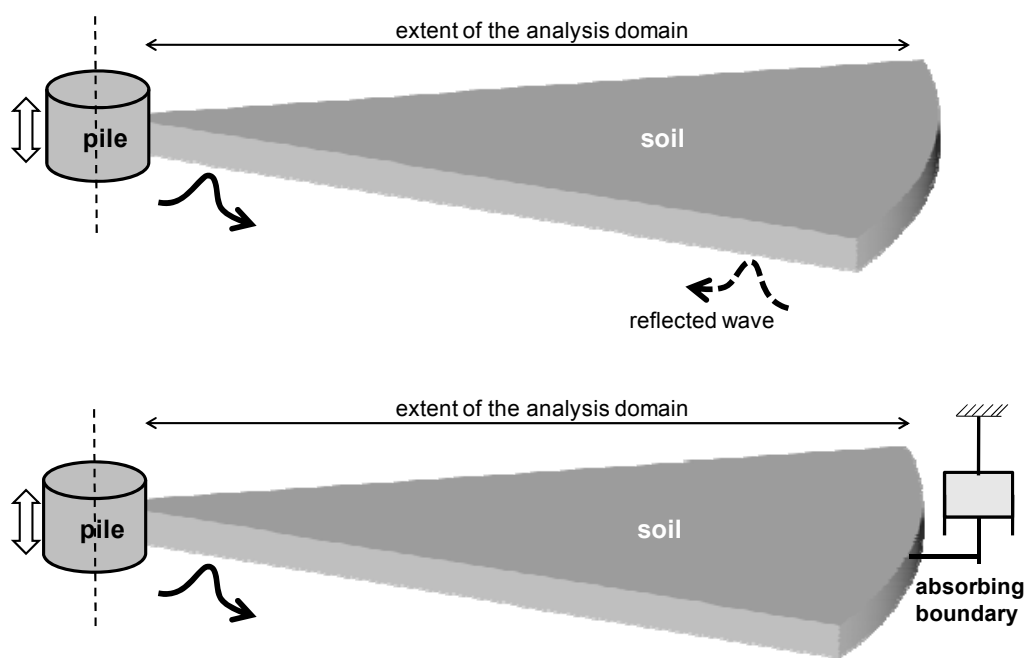

Figure 5.2. Use of radiation dashpot at the outer boundary of the continuum analysis domain.

The outer boundary of the near field is considered as the inner boundary of the far-field domain. The analytical solution by Novak et al. (1978) yields the coefficient of a radiation dashpot representing a soil disk of infinite extent when excited at its inner boundary (the 
location of the shaft wall). Thus, there is an analogy between the shaft wall in the Novak et al. (1978) solution and the outer boundary of the near field in the present approach. Following the Novak et al. (1978) solution, the radiation dashpot coefficient for the outer boundary of the near field for high frequency motion (which is prevalent in pile driving) is given by:

$$
c_{\mathrm{FF}}=\frac{G_{\max }}{V_{\mathrm{s}}}=\rho \cdot V_{\mathrm{s}}
$$

where $G_{\max }$ is the maximum (i.e., small-strain) shear modulus of the soil and $V_{\mathrm{s}}$ is the soil shear wave velocity. It can be seen that the dashpot coefficient is independent of the radius of the boundary to which the dashpot is attached. It is interesting to note that eq. (5.5) is also valid for 1-D wave propagation under plane strain conditions.

The absorbing boundary formulations available in finite difference or finite element analysis software (e.g. FLAC, ABAQUS) consist only of radiation dashpots. This approach is not suitable for the present 1-D formulation. The motion involved in the problem of pile driving is highly transient. There is the initial stage of large rise in displacement followed by a stage of oscillations with continuously decreasing amplitude with time. After all vibrations have been dissipated, we should recover the static solution, i.e., a permanent soil displacement with magnitude decreasing with increasing radial distance, caused by a residual shear stress on the pile shaft. If no spring is placed at the outer boundary of the analysis domain, the soil disk will become level and unstressed with all final displacements being equal to the displacement at the pile shaft (Fig. 5.3) because, in the absence of a reacting spring, no stress will remain at the outer boundary after dissipation of vibrations (the dashpot provides no static resistance since its reaction is proportional to the velocity of motion). 


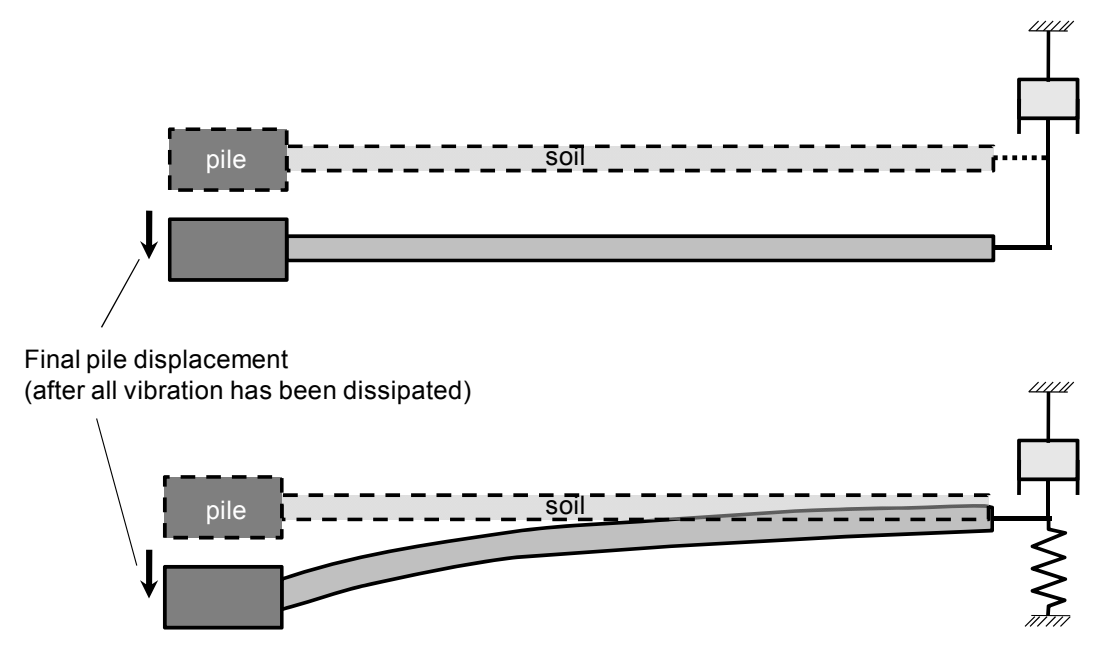

Figure 5.3. The pring at the outer boundary of the analysis domain generates a displacement profile consistent with what is expected at the end of driving.

A static solution was derived by Randolph and Wroth (1978) by first establishing an expression for the radius of influence $r_{\mathrm{m}}$ around the pile (the so-called "magical" radius) based on 2-D finite element analysis. The soil displacement at $r \geq r_{\mathrm{m}}$ is assumed to be zero by definition. If the soil profile is homogeneous, the radius of influence is simply a function of the pile length $L$ and the soil Poisson's ratio $v$ :

$$
r_{\mathrm{m}}=2.5 L(1-v)
$$

Eq. (5.6) can be extended to the case of a nonhomogeneous soil profile (Fleming et al. 1992):

$$
r_{\mathrm{m}}=\left\{0.25+\left[2.5(1-v) \frac{G_{\mathrm{M}}}{G_{\mathrm{L}}}-0.25\right] \frac{G_{\mathrm{L}}}{G_{\mathrm{b}}}\right\} L
$$

where $G_{b}$ is the shear modulus of the bearing layer, $G_{L}$ is the soil shear modulus of the soil immediately above the bearing layer, and $G_{M}$ is the shear modulus value at the middle of the soil that lies above the bearing layer. The static solution of Randolph and Wroth (1978) yields the spring constant $k_{\mathrm{FF}}$ : 


$$
k_{\mathrm{FF}}=\frac{G_{\mathrm{max}}}{r_{\mathrm{f}}} \frac{1}{\ln \left(r_{\mathrm{m}} / r_{\mathrm{f}}\right)}
$$

Eq. (5.8) gives a constant spring stiffness value that is valid only after all vibrations in the soil have dissipated. On the other hand, if the pile and soil motion were harmonic, $k_{\mathrm{FF}}$ would be given by the Novak et al. (1978) solution, which is used by Randolph and Simons (1986):

$$
k_{\mathrm{FF}}^{*}=1.375 \frac{G_{\mathrm{max}}}{\pi r_{\mathrm{f}}}
$$

The actual spring stiffness varies with time during the transient soil response caused by pile driving (probably between the values given by eq. 5.8 and eq. 5.9). Unfortunately, there is no closed-form solution that would give $k_{\mathrm{FF}}$ in accordance with the real dynamic conditions. Here, we will use the static solution (eq. 5.8) because $k_{\mathrm{FF}}$ does not impact greatly the dynamic response and the wave absorption (which is fully controlled by the dashpot) but controls the static residual stress acting on the pile shaft after vibrations have dissipated. If $r_{f}$ is set to $0.102 r_{\mathrm{m}}, k_{\mathrm{FF}}$ and $k_{F F}^{*}$ become equal.

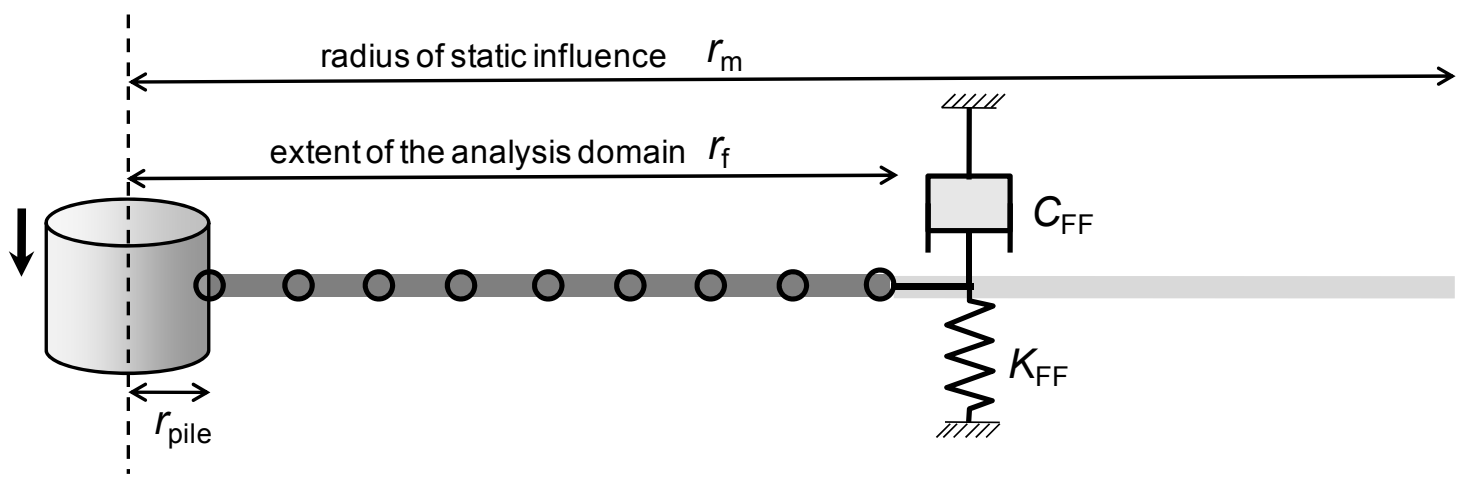

Figure 5.4. Discretized near-field domain with spring and dashpot representing the far-field soil attached to the outer boundary.

Having established the boundary conditions at the outer edge of the analysis domain as indicated in Fig. 5.4, eq. (5.4) for the outmost $\left(N^{\text {th }}\right)$ point becomes: 
$w_{N_{s}}^{(t+\Delta t)}=\frac{\Delta r \Delta t^{2}}{\rho \Delta r+\Delta t 2 c_{\mathrm{FF}}}\left[G \frac{2 w_{N_{s}-1}^{(t)}-2 w_{N_{s}}^{(t)}}{\Delta r^{2}}-\frac{G}{r_{N_{s}}} \frac{w_{N_{s}-1}^{(t)}-w_{N_{s}}^{(t)}}{\Delta r}-\frac{2 c_{\mathrm{FF}}}{\Delta r} \frac{w_{N_{s}}^{(t-\Delta t)}}{\Delta t}-\frac{2 k_{\mathrm{FF}}}{\Delta r} w_{N_{s}}^{(t)}+\frac{\rho}{\Delta t^{2}}\left(2 w_{N_{s}}^{(t)}-w_{N_{s}}^{(t-\Delta t)}\right)\right]$

To test the validity of the proposed absorbing boundary formulation, we will perform simple simulations of wave transmission through the near-field disk. In the following examples, the shear modulus $G$ is equal to $100000 \mathrm{kPa}$ and the soil density is $1.8 \mathrm{t} / \mathrm{m}^{3}$ (shear wave velocity $V_{s}=236 \mathrm{~m} / \mathrm{s}$ ). The pile radius is $0.15 \mathrm{~m}$, the extent of the analysis domain $r_{\mathrm{f}}$ is $3.15 \mathrm{~m}$, and the number of soil nodes is $30(\Delta r=0.1 \mathrm{~m})$. Assuming pile length equal to $10 \mathrm{~m}$ and soil Poisson's ratio equal to 0.25 , eq. (5.6) yields a radius of static influence $r_{\mathrm{m}}$ equal to $18.75 \mathrm{~m}$. The time increment $\Delta t$ is set equal to $0.00003 \mathrm{~s}$ in all examples. $\Delta t$ must be smaller than $\Delta r / V_{\mathrm{s}}$ to ensure numerical stability during the integration of the equations of motion (Cook et al. 2002). It should be noted that the critical time step that is required in the 1-D dynamic pile analysis is much smaller since the shear wave velocity of the pile material is much larger than that of the soil.

In the first example (Fig. 5.5), the applied motion at the inner boundary (the location of the pile shaft) is harmonic with constant amplitude and period $T=0.02 \mathrm{~s}$. The spring stiffness is calculated using eq. (5.8). When $c_{\mathrm{FF}}$ takes the value given by eq. (5.5), the waves are fully absorbed at the outer boundary as attested by the continuously decreasing motion amplitude with radial distance and the unaltered pattern of the incident waves with time. 

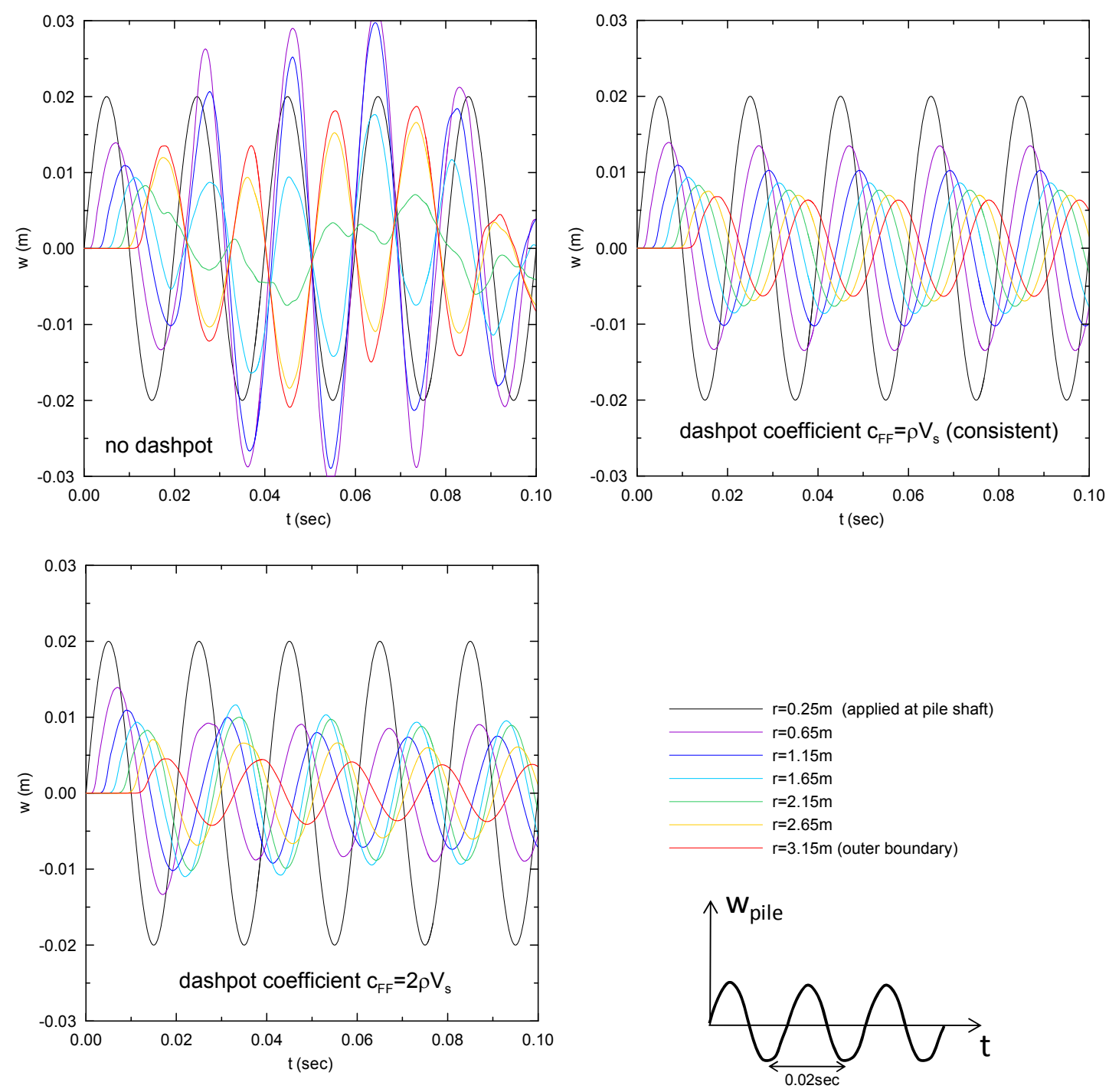

Figure 5.5. Soil response at different radial distances due to harmonic oscillation of the pile shaft.

Fig 5.5 shows that, in the absence of a radiation dashpot, the wave is fully reflected at the outer boundary, generating a standing wave inside the soil disk. If the dashpot coefficient is set to twice the value yielded by eq. (5.5), we see that the wave is partially reflected, with local increase in motion amplitude midway between the inner and outer boundaries. The same conclusions can be drawn from the example presented in Fig. 5.6. In this example, the input motion is of transient character and defined by a half sinusoidal pulse. Full and partial reflection is observed in the cases of no dashpot and of dashpot coefficient larger than the value for a consistent boundary. 

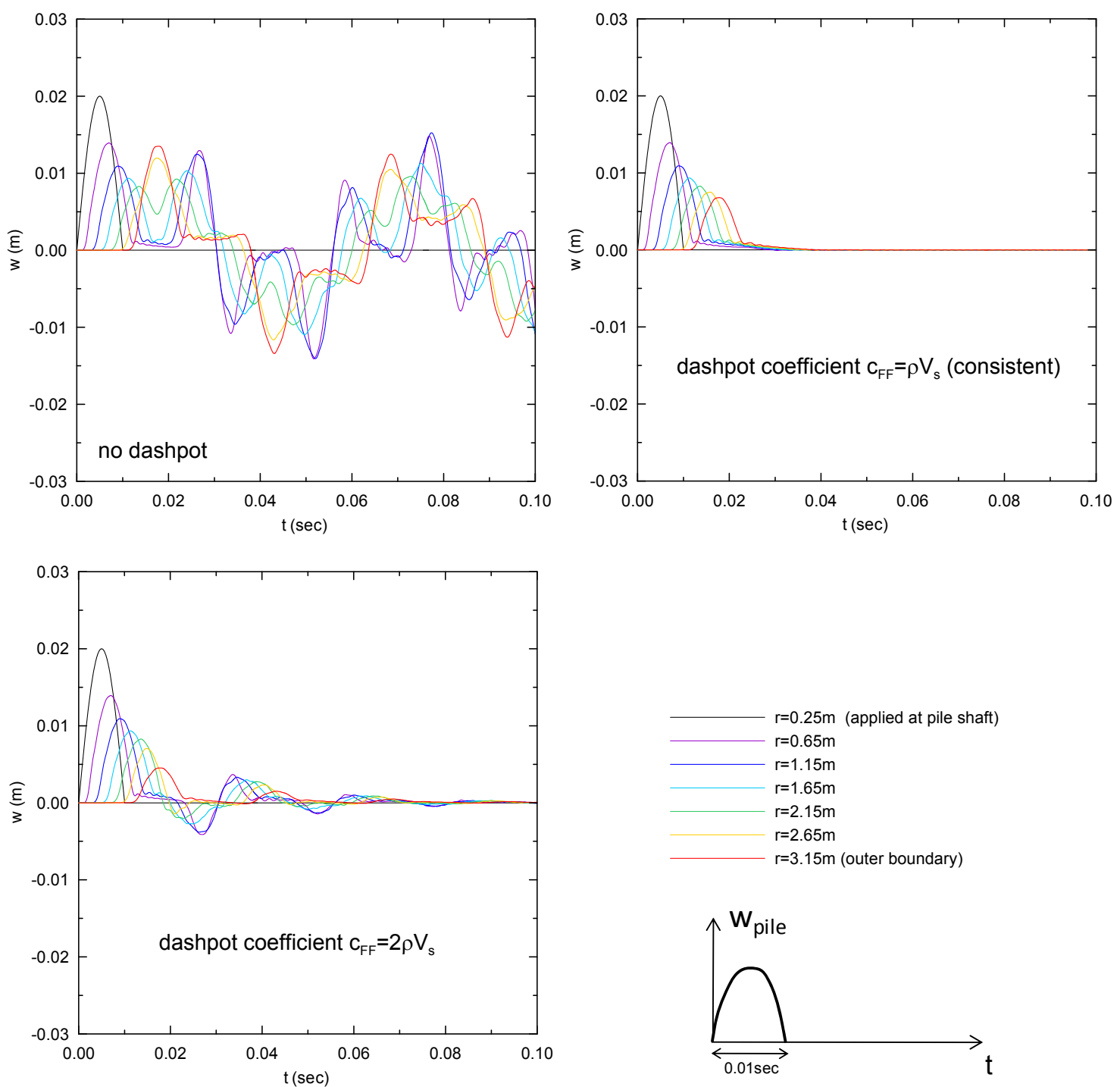

Figure 5.6. Soil response at different radial distances due to half sinusoidal pulse (no permanent displacement) at the pile shaft.

If the dashpot coefficient is set to the far-field consistent value $\left(\rho V_{s}\right)$, the outer boundary is 'transparent' to the incident wave, i.e. the soil motion practically vanishes once the wave reaches the outer boundary. The soil motion does not cease immediately after the passing of the wave through a given point in the soil disk, but a small residual portion of it dissipates progressively with time. This is a deviation from the theoretically exact response and is caused by numerical error inherent to the numerical solution. The example in Fig. 5.7 demonstrates the function of the outer boundary spring. The input pulse at the inner boundary consist of a sinusoidally increasing displacement up to a value of $2 \mathrm{~cm}$ at $t=0.005 \mathrm{~s}$ 
and remaining unchanged thereafter. The dashpot coefficient is that defined in eq. (5.5). If no spring is placed at the outer boundary, all nodal displacements converge with time to the final input displacement magnitude. In that case, all shear strains and, consequently, stresses vanish at the end of the analysis. In contrast, the solution converges to the static solution if the spring defined in eq. (5.8) is placed at the outer boundary. The pile shaft motion in pile driving analysis is a combination of the motions used in the examples of Figs 5.6 and 5.7 .
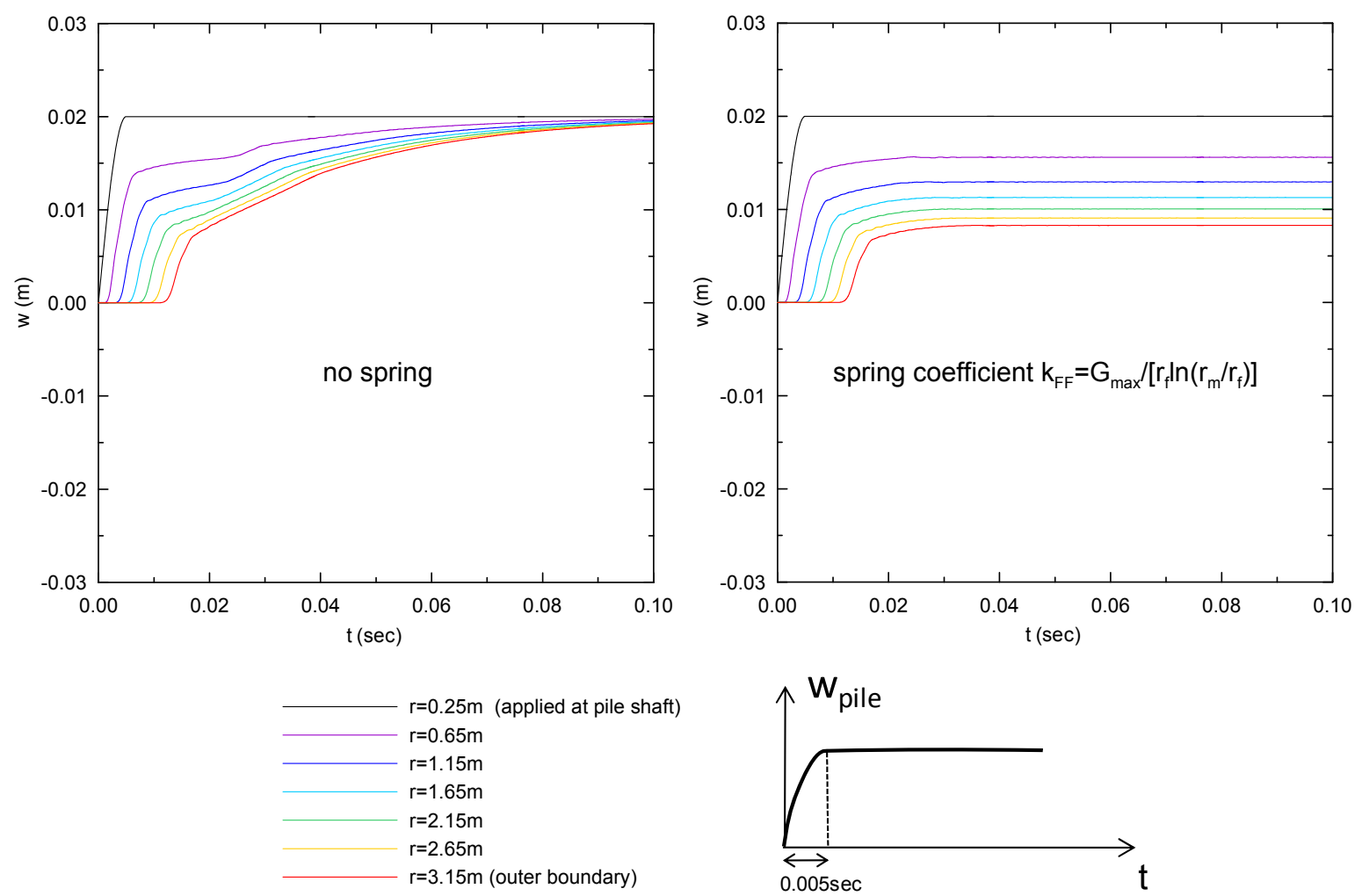

Figure 5.7. Soil response at different radial distances due to quarter sinusoidal pulse with permanent displacement at the pile shaft.

\subsubsection{Soil nonlinearity in soil disk formulation}

The formulation presented in the previous section was based on the assumption that the soil is elastic. The major advantage of the continuum approach is that it can handle effectively soil nonlinearity and hysteresis by allowing the implementation of any constitutive model for 
the soil. Here, we will assume that the soil follows a "hyperbolic" stress-strain law in rate form:

$$
\dot{\tau}=\frac{G_{\max }}{\left(1+b_{\mathrm{f}} \frac{\left|\tau-L I \cdot \tau_{\mathrm{rev}}\right|}{(L I+1) \times\left|\operatorname{sgn} \cdot \tau_{\mathrm{f}}-\tau\right|}\right)^{2}} \dot{\gamma}
$$

where $\tau_{\mathrm{f}}$ is the shear strength of the soil in simple shear conditions, $\tau_{\mathrm{rev}}$ is the shear stress at the last stress reversal, and $\mathrm{LI}$ is the loading index parameter that takes the values 0 for virgin loading and 1 for subsequent unloading and reloading. The variable sgn is the sign of the strain rate $\dot{\gamma}$. The shear modulus $G$ decreases with shear strain $\gamma$ from its maximum (small-strain) value $G_{\max }$ and approaches zero at very high shear strain levels; $\tau_{f}$ is approached asymptotically as the shear strain increases. Every time the shear strain direction changes (strain or stress reversals), the soil shear modulus $G$ becomes again equal to $G_{\max }$. The parameter $b_{\mathrm{f}}$ controls the rate of degradation of the shear modulus. The shear modulus degradation is controlled mainly by the plasticity index $(P I)$ of the soil (Vucetic and Dobry 1991). The experimental curves by Vucetic and Dobry (1991) can be approximated by eq. (5.11) with $b_{\mathrm{f}}$ calculated using the following equation:

$$
b_{\mathrm{f}}=5 \exp (-0.05 P I)
$$

Comparison between secant shear modulus predictions using eqs. (5.11) and (5.12) and the experimental curves by Vucetic and Dobry (1991) is shown in Fig. 5.8. The stress-strain relationship of eq. (5.11) can be seen as an extension of the Fahey-Carter model to cyclic loading conditions with the exponent used in Fahey and Carter (1993) always equal to unity.

Fig. 5.9 plots the cyclic stress-strain response predicted by eqs. (5.11) and (5.12) for two extreme values of the degradation parameter $b_{\mathrm{f}}$. It can be seen that the proposed stress-strain relationship reproduces realistically the soil behavior during unloading and reloading cycles, producing symmetric hysteresis loops. 


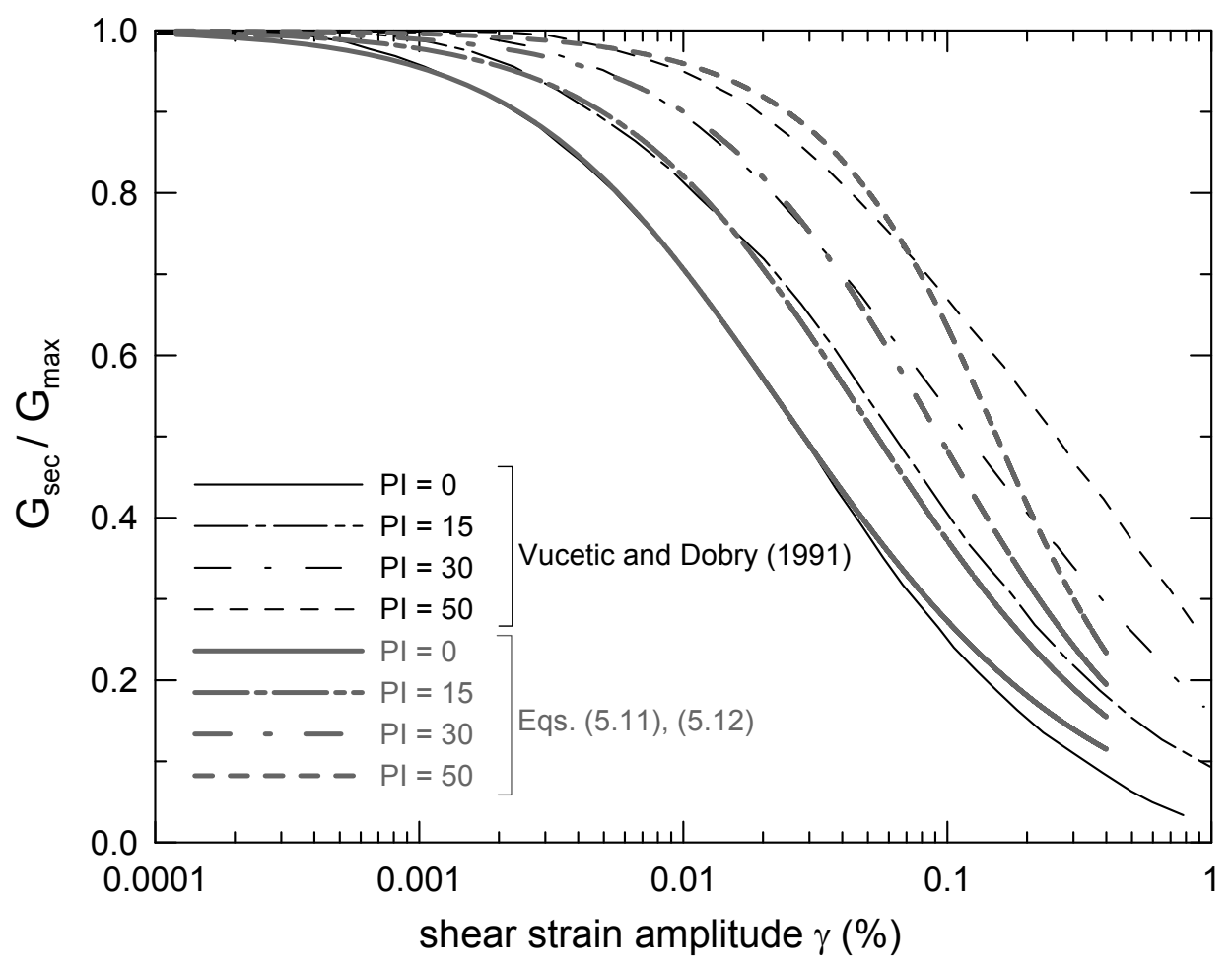

Figure 5.8. Secant shear strain modulus as a function of shear strain: experimental curves by Vucetic and Dobry (1991) compared with predictions using eq. (5.11) and (5.12).

In the case of nonlinear soil, we cannot use eq. (5.2) because the secant shear modulus changes continuously with time during the highly transient soil response. Thus, we need to consider explicitly the shear stress $\tau$ in the solution formulation. The shear stress will be calculated at each time increment using eq. (5.11). Using central finite difference approximation, the first derivative of the shear stress in eq. (5.1) for the node $i$ is expressed as

$$
\frac{\partial \tau}{\partial r} \approx \frac{\tau_{L, i}^{(t)}-\tau_{R, i}^{(t)}}{\Delta r}
$$

where $\tau_{\mathrm{L}}$ and $\tau_{\mathrm{R}}$ are the shear stress at the mid-points of the segments on the left and on the right of node $i$. Each pair of adjacent nodes defines a segment where the strain and, consequently, the stress are assumed to be constant. The shear stress in each segment at each time increment is calculated incrementally based on eq. (5.11): 


$$
\begin{gathered}
\tau_{L, i}^{(t)}=\frac{G_{\max }}{\left(1+b_{\mathrm{f}} \frac{\left|\tau_{L, i}^{(t-\Delta t)}-L I_{\mathrm{L}, \mathrm{i}} \cdot \tau_{L, \mathrm{rev}, i}\right|}{\left(L I_{\mathrm{L}, \mathrm{i}}+1\right) \times\left|\operatorname{sgn} \cdot \tau_{\mathrm{f}}-\tau_{L, i}^{(t-\Delta t)}\right|}\right)^{2}} \frac{\left(w_{i-1}^{(t)}-w_{i-1}^{(t-\Delta t)}\right)-\left(w_{i}^{(t)}-w_{i}^{(t-\Delta t)}\right)}{\Delta r}+\tau_{L, i}^{(t-\Delta t)} \\
\tau_{R, i}^{(t)}=\frac{G_{\max }}{\left(1+b_{\mathrm{f}} \frac{\left|\tau_{R, i}^{(t-\Delta t)}-L I_{\mathrm{R}, i} \cdot \tau_{R, \mathrm{rev}, i}\right|}{\left(L I_{\mathrm{R}, \mathrm{i}}+1\right) \times\left|\operatorname{sgn} \cdot \tau_{\mathrm{f}}-\tau_{R, i}^{(t-\Delta t)}\right|}\right)^{2} \frac{\left(w_{i}^{(t)}-w_{i}^{(t-\Delta t)}\right)-\left(w_{i+1}^{(t)}-w_{i+1}^{(t-\Delta t)}\right)}{\Delta r}+\tau_{R, i}^{(t-\Delta t)}}
\end{gathered}
$$

The shear stress right at the position of node $i$ is calculated in a similar way:

$$
\tau_{i}^{(t)}=\frac{G_{\max }}{\left(1+b_{\mathrm{f}} \frac{\left|\tau_{i}^{(t-\Delta t)}-L I_{\mathrm{i}} \cdot \tau_{\mathrm{rev}, \mathrm{i}}\right|}{\left(L I_{\mathrm{i}}+1\right) \times\left|\operatorname{sgn} \cdot \tau_{\mathrm{f}}-\tau_{i}^{(t-\Delta t)}\right|}\right)^{2}} \cdot \frac{\left(w_{i-1}^{(t)}-w_{i-1}^{(t-\Delta t)}\right)-\left(w_{i+1}^{(t)}-w_{i+1}^{(t-\Delta t)}\right)}{2 \Delta r}+\tau_{i}^{(t-\Delta t)}
$$

Having established the shear stresses on both sides of node $i$, eq. (5.1) is approximated by

$$
\frac{\tau_{L, i}^{(t)}-\tau_{R, i}^{(t)}}{\Delta r}-\frac{\tau_{i}^{(t)}}{r_{i}}=\rho \frac{w_{i}^{(t+\Delta t)}-2 w_{i}^{(t)}+w_{i}^{(t-\Delta t)}}{\Delta t^{2}}
$$

Provided that we know the displacements at all the nodes at times $(t)$ and $(t-\Delta t)$, we can calculate the displacement at time $(t+\Delta t)$ at each node $i$ by solving eq. (5.14) with respect to $w_{i}^{(t+\Delta t):}$

$$
w_{i}^{(t+\Delta t)}=\frac{\Delta t^{2}}{\rho}\left[\frac{\tau_{L, i}^{(t)}-\tau_{R, i}^{(t)}}{\Delta r}-\frac{\tau_{i}^{(t)}}{r_{i}}\right]+2 w_{i}^{(t)}-w_{i}^{(t-\Delta t)}
$$




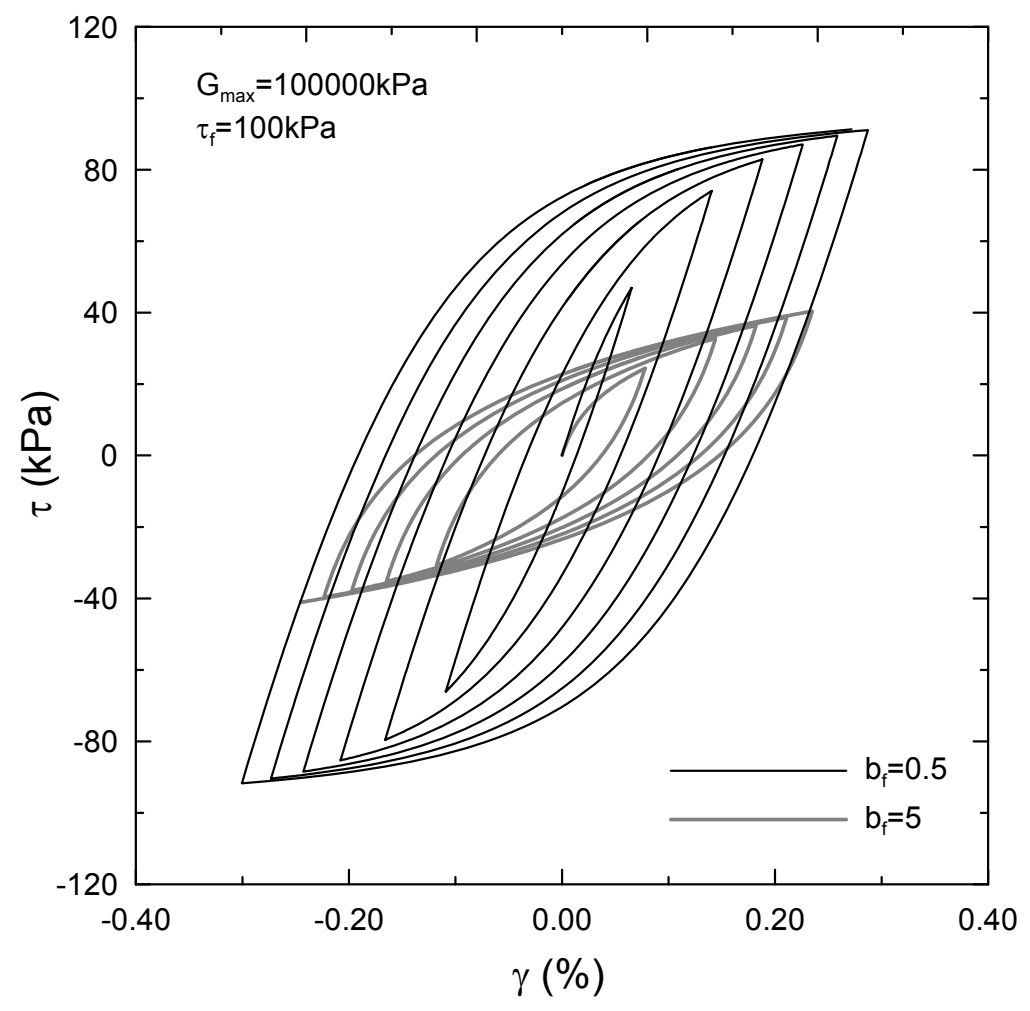

Figure 5.9. Stress-strain loops predicted by eq. (5.11) for two different values of the degradation parameter $b_{\mathrm{f}}$.

The main output of the soil disk solution is the soil reaction at the pile shaft wall (i.e., at the inner boundary of the near-field continuum domain). This reaction is taken as the shear stress in the soil segment between nodes 1 and 2:

$$
\tau_{\mathrm{S}}^{(t)}=\frac{G_{\max }}{\left(1+b_{\mathrm{f}} \frac{\left|\tau_{S}^{(t-\Delta t)}-L I_{\mathrm{S}} \cdot \tau_{S, \mathrm{rev}}\right|}{\left(L I_{\mathrm{S}}+1\right) \times\left|\operatorname{sgn} \cdot \tau_{\mathrm{f}}-\tau_{S}^{(t-\Delta t)}\right|}\right)^{2}} \frac{\left(w_{1}^{(t)}-w_{1}^{(t-\Delta t)}\right)-\left(w_{2}^{(t)}-w_{2}^{(t-\Delta t)}\right)}{\Delta r}+\tau_{\mathrm{S}}^{(t-\Delta t)}
$$

from which the shear force acting on the shaft can be computed as

$$
R_{\mathrm{s}}^{(t)}=\tau_{\mathrm{S}}^{(t)} \Pi_{\text {pile }} \Delta L
$$


where $\Pi_{\text {pile }}$ is the perimeter of the pile cross-section and $\Delta \mathrm{L}$ is the length of the pile segment for which soil reactions are calculated.

We complete the shaft reaction model by introducing a rheological model that represents the shear band along the shaft wall. Here, we adopt the rheological model proposed by Randolph and Simons (1986). It consists of a plastic slider and a viscous dashpot connected in parallel placed between the first node of the continuous near field and the corresponding pile segment (Fig. 5.10). The strength of the plastic slider is equal to the static unit limit shaft resistance $q_{\mathrm{sL}}$. As long as the stress $\tau_{\mathrm{s}}$ is smaller than $q_{\mathrm{sL}}$, no sliding occurs and $\tau_{\mathrm{s}}$ is transmitted to the pile segment. Sliding initiates once $\tau_{\mathrm{s}}=q_{\mathrm{sL}}$, at which point the viscous dashpot becomes activated. The reaction of the viscous dashpot is a power function of the relative velocity between the pile and the first node of the near field component. The total (static+viscous) resistance of the rheological model is given by

$$
\tau_{\mathrm{sf}}=q_{\mathrm{sL}}\left(1+m_{\mathrm{s}}\left(\dot{w}_{\mathrm{pile}}-\dot{w}_{1}\right)^{n_{\mathrm{s}}}\right)
$$

where $m_{\mathrm{s}}$ and $n_{\mathrm{s}}$ are input parameters. Based on data by Coyle and Gibson (1970), Dayal and Allen (1975), Heerema (1979), Litkouhi and Poskitt (1980), the parameter $n_{\mathrm{s}}$ is generally in the 0.2 to 0.5 range (but for most cases is in the vicinity of 0.2 ), while $m_{\mathrm{s}}$ takes values from 0.3 to 0.5 for sands and 2.0 to 3.0 for clays (velocity is in units of $\mathrm{m} / \mathrm{s}$ ). The strength of soil in the near field needs to be consistent with the strength of the shear band. Thus, the strength $\tau_{\mathrm{f}}$ inside the continuum is set to be also a function of the relative velocity:

$$
\tau_{\mathrm{f}}=\tau_{\mathrm{f}, \mathrm{stat}}\left(1+m_{\mathrm{s}}\left(\dot{w}_{\text {pile }}-\dot{w}_{1}\right)^{n_{\mathrm{s}}}\right)
$$

The static soil strength of the soil $\tau_{\text {f,stat }}$ is set to be slightly higher than the actual soil shear strength because the hyperbolic stress-strain relationship of eq. (5.11) approaches the limit shear stress $\tau_{f}$ asymptotically. The value of $\tau_{\mathrm{f}, \text { stat }}$ is chosen so that the slider becomes activated at a shear strain level at the first near-field segment in the $5 \%$ to $10 \%$ range. This is the shear strain range within which soils are expected to reach "failure" under simple shear conditions. In the case of clays and silts, $\tau_{\mathrm{f}, \text { stat }}$ is defined by the following equation

$$
\tau_{\mathrm{f}, \mathrm{stat}}=R_{\mathrm{F}} S_{\mathrm{u}}
$$




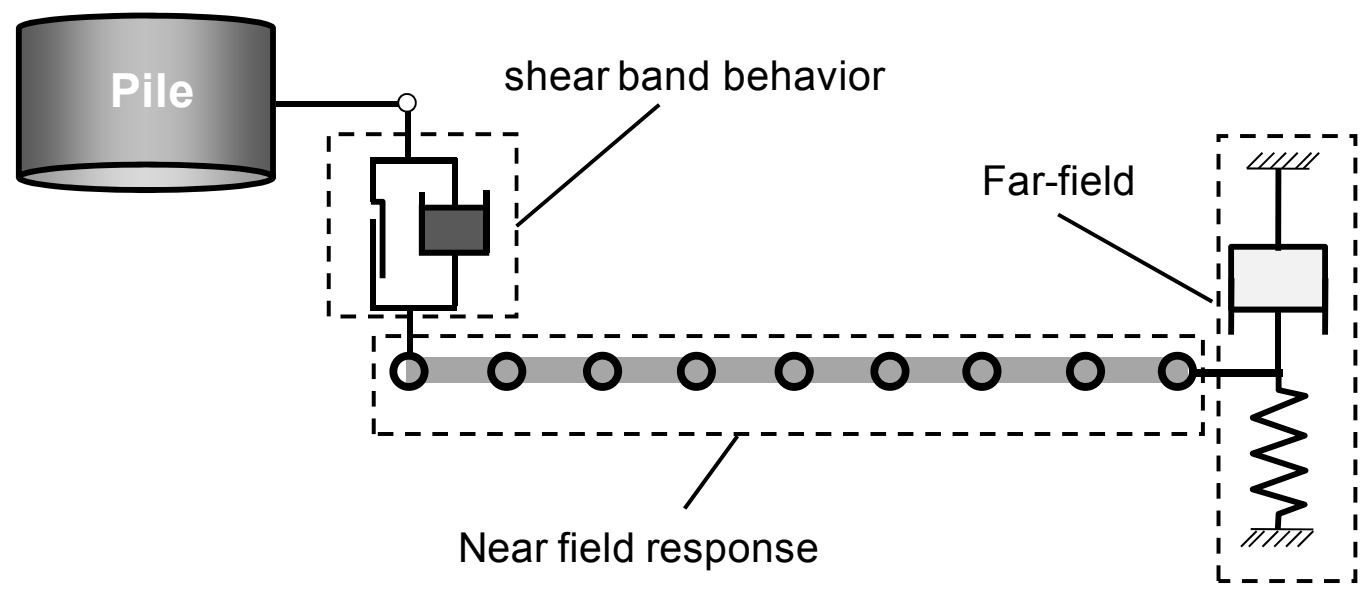

Figure 5.10. Proposed shaft reaction model consisting of three parts: continuous near field, far-field consistent spring and radiation dashpot, and rheological shear band model at the soil-pile interface.

The factor $R_{\mathrm{F}}$ is a function of the parameter $\mathrm{b}_{\mathrm{f}}$ (which, in turn, is a function of $\mathrm{PI}$ ):

$$
R_{\mathrm{F}}=\frac{1}{1-0.015 b_{\mathrm{f}}^{1.5}}
$$

In the case of sands, $\tau_{\mathrm{f}, \mathrm{stat}}$ is defined by the following equation:

$$
\tau_{\mathrm{f}, \mathrm{stat}}=1.2 n_{\mathrm{F}} q_{\mathrm{sL}}
$$

The factor $n_{\mathrm{F}}$ depends on the roughness of the shaft wall. It takes the values 1.0 and 1.22 for piles made of concrete and mild steel, respectively. In reality, the sand shear strength will decrease with radial distance from the pile shaft because of the decrease of mean effective stress with $r$. The stress changes around the pile shaft due to the installation of the pile dissipate with distance from the pile. Here, for simplicity, we assume that the strength is constant throughout the near field.

The strain rate affects only the soil shear strength. The small- strain soil stiffness is independent of the rate of strain (Tatsuoka et al. 1997). Because of the dependence of the ultimate strength on strain rate, the model predicts a small increase in the secant stiffness 
with strain rate in the intermediate range of strains. Brown et al. (2006) performed rapid and static pile load tests on an instrumented drilled shaft installed in a clayey soil profile. Their data shows that, although the pile velocity influences the limit shaft resistance, the shear stress mobilized on the shaft at pre-failure loads shows small to no increase with pile velocity.

The extent of the near-field domain, $r_{\mathrm{f}}$, needs to be a certain fraction of the radius of influence $r_{\mathrm{m}}$. The radii $r_{\mathrm{f}}$ and $r_{\mathrm{m}}$ cannot be equal because this will negate the effect of the far field spring and radiation dashpot. The near-field domain needs to extend only up to the point where the shear strains become small enough for the soil nonlinearity to be negligible. We will set the ratio $r_{\mathrm{f}} / r_{\mathrm{m}}$ to be in the 0.2 to 0.5 range. The discretization of the near-field domain should be fine enough so that the combined length of five consecutive segments is not smaller than the wavelength of the shear waves propagating through the domain.

Once slippage initiates, the displacement of the first node of the near-field component (node 1) and the pile displacement become different. The displacement of node 1 then ceases to be a known input, and its value needs to be calculated as part of the solution. During slippage in the plastic slider, the dynamic equilibrium equation at node 1 can be written as

$$
\frac{\tau_{\mathrm{sf}}^{(t)}-\tau_{R, 1}^{(t)}}{\Delta r}-\frac{\tau_{\mathrm{sf}}^{(t)}+\tau_{R, 1}^{(t)}}{2 r_{1}}=\rho \frac{w_{1}^{(t+\Delta t)}-2 w_{1}^{(t)}+w_{1}^{(t-\Delta t)}}{\Delta t^{2}}
$$

Eq. (5.24) can be solved for $w_{i}^{(t+\Delta t)}$ :

$$
w_{1}^{(t+\Delta t)}=\frac{\Delta t^{2}}{\rho}\left(\frac{\tau_{\mathrm{sf}}^{(t)}-\tau_{R, 1}^{(t)}}{\Delta r}-\frac{\tau_{\mathrm{sf}}^{(t)}+\tau_{R, 1}^{(t)}}{2 r_{1}}\right)+2 w_{1}^{(t)}-w_{1}^{(t-\Delta t)}
$$

Slippage in the slider stops and the near-field rejoins the pile once the relative velocity between the pile segment and node 1 becomes again zero.

The proposed model has similarities with the shaft reaction model of Holeyman et al. (1996), although our formulation does not consider ring soil elements with the soil mass lumped at the center of these elements. Our formulation is based on discretizing and solving the fundamental dynamic equilibrium equation (eq. 5.1), which, for the present problem, is equivalent to the wave equation for shear waves in axisymmetric conditions. The equilibrium 
is expressed in terms of shear stress (instead of secant forces), and the mass is assumed continuous (not lumped). The new model seeks to eliminate the limitations of the Holeyman et al. (1996) model identified and described in Chapter 4. The spring and radiation dashpot placed at the outer boundary of the near-field domain are consistent with the far-field conditions, with the spring constant being a function of the extent of the near-field domain and the radius of pile influence. We also added a rheological component between the nearfield domain and the pile in order to more accurately represent the mechanics of the shear banding and slippage at the pile shaft wall. Our formulation allows for the consideration of as many soil disks as there are lumped masses discretizing the pile. For this reason, the pile does not need to be assumed to be rigid. Each soil disk can have different properties and input parameters. Any interaction between the soil disks is neglected. In reality, the motion of the soil at a given depth influences the motion of the soil immediately above and below that depth. Moreover, primary waves will emanate from the pile shaft and will propagate through the soil in an oblique direction. Another factor influencing the soil deformation in reality is arching, i.e. the oblique transfer of stress through the soil. These phenomena cannot be captured by the 1-dimensional approach we propose; capturing these phenomena would require use of complicated and computationally expensive 2-dimensional solution schemes. . Taking into account soil interaction between different elevations would require 2-dimensional discretization of the surrounding soil mass.

\subsection{Proposed base reaction model}

A complete base reaction model needs to take into account the nonlinear soil response under the pile base, the rate effect on base resistance, and distinguish between the different types of damping. As discussed in Chapter 4, a continuum approach similar to the one used for the shaft reaction model may not be suitable for the base reaction model. This is because, for the base, the development of limit resistance cannot be decoupled from the mechanics of the near-field. In addition, the base reaction problem is 2-dimensional; modeling the soil as a series of lumped masses extending below the pile base would constitutes only a rough approximation with no real advantage over simpler rheological (noncontinuum) models. The predicted soil reaction for very small base velocities (quasistatic conditions) must be consistent with the static load-settlement response that would 
have been observed in static pile load tests. This is hard to achieve with a continuum approach, such as that proposed by Holeyman (1988).

The proposed model consists of a nonlinear spring connected in parallel to a radiation dashpot (Fig. 5.11). The total base reaction is the sum of the spring reaction $R_{\mathrm{b}}{ }^{(S)}$ and the radiation dashpot reaction $R_{\mathrm{b}}{ }^{(\mathrm{D}) \text { : }}$

$$
R_{\mathrm{b}}=R_{\mathrm{b}}^{(S)}+R_{\mathrm{b}}^{(D)}=R_{\mathrm{b}}^{(S)}+C_{\mathrm{b}} \dot{\mathrm{w}}_{\mathrm{b}}
$$

where $\dot{w}_{\mathrm{b}}$ is the velocity of the pile base and $C_{\mathrm{b}}$ is the radiation damping constant. The nonlinear spring follows a hyperbolic-type load-settlement relationship:

$$
\dot{R}_{\mathrm{b}}^{(S)}=\frac{K_{\mathrm{b}, \max }}{\left(1+b_{\mathrm{fb}} \frac{\left|R_{\mathrm{b}}-L I \cdot R_{\mathrm{b}, \mathrm{rev}}\right|}{(L I+1) \times\left|\operatorname{sgn} \cdot R_{\mathrm{bf}}-R_{\mathrm{b}}\right|}\right)^{2}} \dot{w}_{\mathrm{b}}
$$

where $K_{\mathrm{b}, \max }$ is the maximum (elastic) base spring stiffness, $R_{\mathrm{bf}}$ is the limit base capacity, $R_{\mathrm{b}, \text { rev }}$ is the spring reaction $R_{\mathrm{b}}{ }^{(S)}$ at the last displacement reversal, and $L /$ is the loading index parameter that takes the values 0 for virgin loading and 1 for subsequent unloading and reloading. The variable sgn is the sign of the rate of base displacement $\dot{w}$, and $b_{\mathrm{f}}$ is a model parameter that controls the rate of degradation of the base spring stiffness. Eq. (5.27) is similar to eq. (5.11) used in the proposed shaft reaction model. Because tensile stresses cannot be transmitted through the soil-pile base interface, $R_{\mathrm{b}}{ }^{(S)}$ is not allowed to take negative values. The base reaction reaches $R_{\mathrm{bf}}$ asymptotically with increasing pile base displacement $w_{\mathrm{b}}$. At points of displacement reversals (unloading and reloading), the spring stiffness is equal to the initial stiffness value $K_{\mathrm{b} \text {,max. }}$ The model captures hysteretic damping with the help of the variables $L I$, sgn, and $R_{\mathrm{b}, \text { rev }}$. The curvature of the base reaction vs. settlement curve depends on the pile type. The settlement required for displacement piles embedded in clay to reach limit base resistance can be as low as $0.1 B-0.2 B$. In contrast, the limit base resistance is practically never reached in the case of drilled shafts (nondisplacement piles). It was found that, for a displacement pile, $b_{f}$ needs to be in the 1.0 to 2.0 range. For drilled shafts, $b_{\mathrm{fb}}$ values lie between 10 and 20 . 
The rate effect on the soil strength is included in the model through $R_{\mathrm{bf}}$. The limit base resistance $R_{\mathrm{bf}}$ is set to be a function of the base velocity, similarly to the limit shaft resistance:

$$
R_{\mathrm{bf}}=Q_{\mathrm{bL}}\left(1+m_{\mathrm{b}}\left(\dot{w}_{\mathrm{b}}\right)^{n_{\mathrm{b}}}\right)
$$

where $m_{\mathrm{b}}$ and $n_{\mathrm{b}}$ are input parameters controlling the rate effect on strength (soil viscosity). They are parameters similar but not necessarily equal to $m_{\mathrm{s}}$ and $n_{\mathrm{s}}$ used in eq. (5.19). Experiments on model piles by Litkouhi and Poskitt (1980) show that the exponent $n_{\mathrm{b}}$ is roughly equal to $n_{\mathrm{s}}$. However, the multiplier $m_{\mathrm{b}}$ can be significantly smaller than $m_{\mathrm{s}}$ in the case of clayey soils, while for sandy soils the parameters are approximately the same). Guidelines on the selection of the soil viscosity parameters will be given in the next section.

For zero base velocity, $R_{\mathrm{bf}}$ is equal to $Q_{\mathrm{bL}}$, which is the limit base capacity under quasi-static loading. $Q_{\mathrm{bL}}$ is equal to the product of the unit limit base resistance $q_{\mathrm{bL}}$ and the pile base area $A_{\mathrm{b}}$. The attainment of $q_{\mathrm{b}}$ requires very large base displacements, especially in the case of nondisplacement piles (drilled shafts). The unit limit base resistance is approximately equal to the cone penetration resistance $q_{c}$ (Salgado 2008).

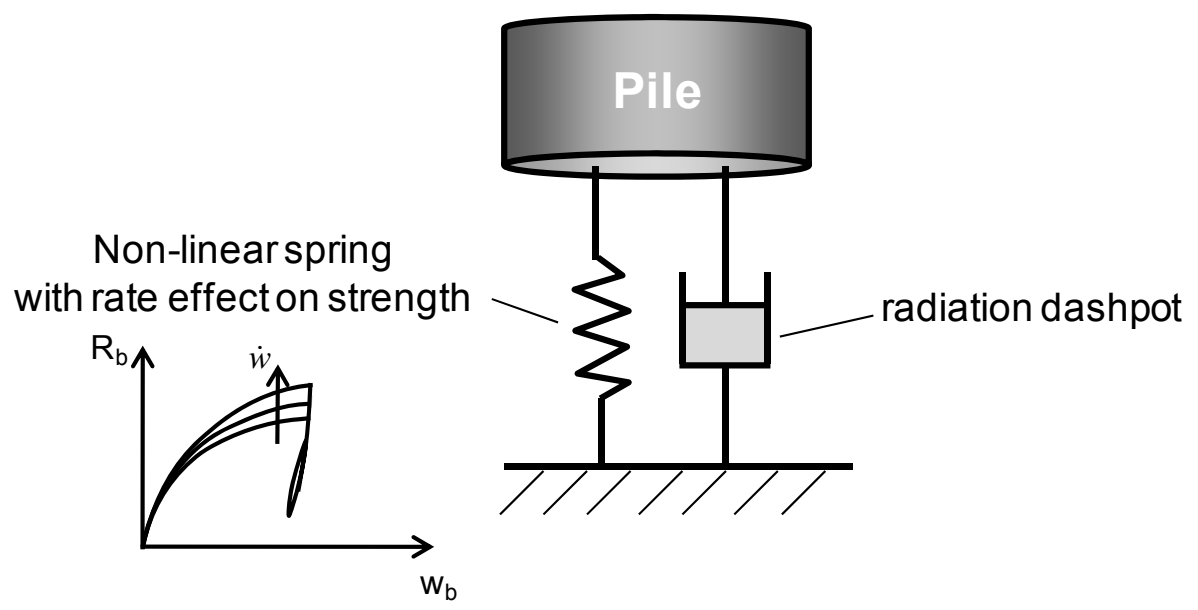

Figure 5.11. Proposed base reaction model consisting of a nonlinear spring and a radiation dashpot.

\subsubsection{Effects of pile base embedment on the stiffness and radiation damping}

Most existing base reaction models use the spring and radiation dashpots coefficients of Lysmer's analog, which are given by the following equations, respectively: 


$$
\begin{aligned}
K_{\text {Lysmer }} & =\frac{4 R G_{\max }}{1-v} \\
C_{\text {Lysmer }} & =\frac{3.4 R^{2}}{1-v} \sqrt{\rho G_{\max }}
\end{aligned}
$$

where $G_{\max }, \rho$ and $v$ are the elastic (small-strain) shear modulus, the density and the Poisson's ratio of the soil, respectively, and $R$ is the pile radius (actual or equivalent). Lysmer's solution was developed for a footing on the surface of a half-space (i.e., a footing with embedment equal to zero). Except during the very early stages of pile driving, the embedment of the pile base is several times the pile width. Existing base reaction models do not take into account any potential effects of embedment on base stiffness and radiation damping. In the context of the present study, we will investigate the embedment effect and propose improved formulas for the base stiffness and dashpot coefficients. For this purpose, we performed a series of analyses of a circular footing, representing the pile base, embedded in an elastic half-space using the finite difference code FLAC. Typical mesh and boundary conditions used in the FLAC simulations are shown in Fig. 5.12. The analysis domain is discretized into four-noded axisymmetric elements (zones, in FLAC's terminology). The lateral and bottom boundaries are absorbing boundaries in dynamic analyses. In static analyses, the nodes at these boundaries were fixed. A circular rigid footing of diameter $B$ is placed at the bottom of a cylindrical well. The well has also diameter $B$ and depth $D$ from the ground surface. The soil is assumed to be linear elastic. The footing is fully attached to the bottom of the well, but its sides are not connected to the wall of the well. The distance between the bottom boundary and footing base is at least $7 B$. The nodes at the wall of the excavation are prevented from moving in the horizontal direction but are free to move in the vertical direction. The choice of this boundary condition at the wall of the well was made because the goal of the FLAC simulations is to derive base stiffness and radiation damping values for pile driving analysis, in which case the presence of the shaft prevents the soil from moving inside the annular space. The vertical degrees of freedom are left free (zero tangential tractions) because limit shaft resistance conditions are reached early during the pile driving analysis and prior to any significant base reaction development. Therefore, any incremental deformation at the pile base will be accompanied by little or no change of tractions along the pile shaft-soil interface. 


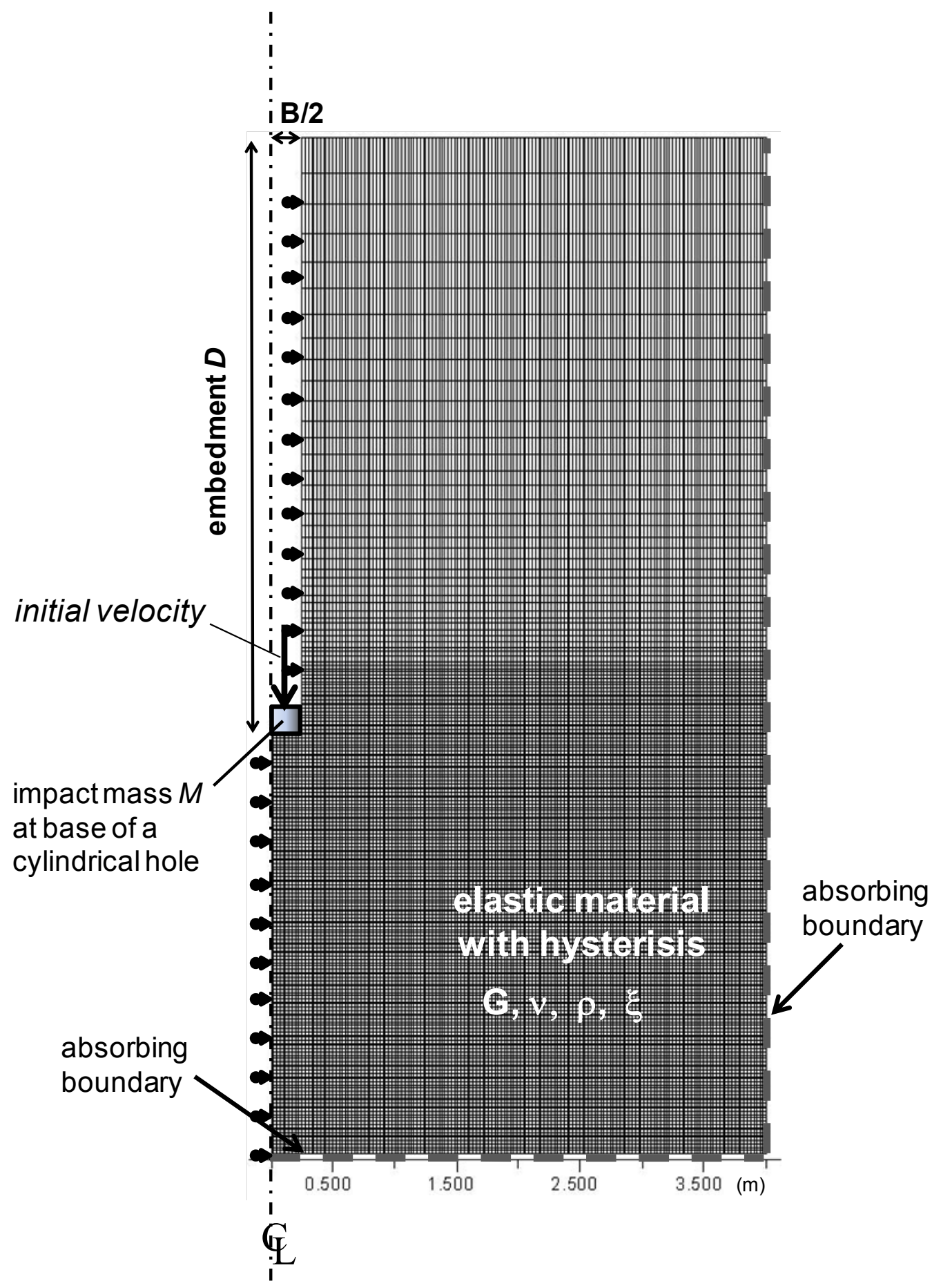

Figure 5.12. Mesh and boundary conditions used in dynamic FLAC analyses of circular footing at the bottom of cylindrical well.

A series of static analyses were performed before the dynamic simulations in order to investigate the effect of embedment on the static base stiffness. Lysmer's solution (eq. 
(5.29)) is valid for both static and dynamic conditions as long as $D=0$. Burland (1970) used finite elements to investigate the effect of embedment on the stiffness of a flexible footing (uniformly loaded area) at the bottom of an unsupported excavation (soil is permitted to move inside the annular space). The results of Burland (1970) refer to the settlement at the center of the flexible footing. Fox (1948) provided an analytical solution for the case with no annular space, i.e., the case in which the space above the flexible footing is filled with soils. Pells and Turner (1978) used finite element results to analyze the same as Burland (1970), but their results refer to the average flexible footing settlement instead of the settlement at the footing center. The problems solved by Fox (1948), Burland (1970) and Pells and Turner (1978) are different from the one considered here (rigid footing in a laterally supported and smooth excavation wall).

FLAC static analyses were performed for Poisson's ratio $v$ values $0.15,0.33$ and 0.49 . The soil shear modulus $G$ was equal to $100000 \mathrm{kPa}$ and the footing diameter was $0.5 \mathrm{~m}$. Analyses were done for embedment ratio values $D / B$ between 0 and 20 . The main output of the numerical simulations was the ratio of the measured footing stiffness $K$ to the stiffness corresponding to zero embedment $K_{\text {emb. }=0}$. This ratio constitutes the static stiffness depth factor $D_{\mathrm{f}}$. The numerical results can be fitted accurately by the following equation

$$
D_{\mathrm{f}}=(1.27-0.12 \ln v)-(0.27-0.12 \ln v) \exp \left[-0.83\left(\frac{D}{B}\right)^{0.826}\right]
$$

Predictions by eq. (5.31) lie between the results of Fox (1948) and Burland (1970) and are slightly larger (up to $3 \%$ difference) than the results of Pells and Turner (1978). They are also roughly $5 \%$ larger that the values presented in Gazetas et al. (1985) for a rigid footing in an unsupported well. An important aspect of eq. (5.31) is that $D_{f}$ takes practically a constant value for $D / B>8$ (Fig. 5.13). This means that the ground surface ceases to influence the base stiffness for intermediate and high embedment ratio values. This ultimate $D_{\mathrm{f}}$ value ranges from 1.36 for $v=0.5$ (saturated clay) to 1.5 for $v=0.15$ (dry sand). 


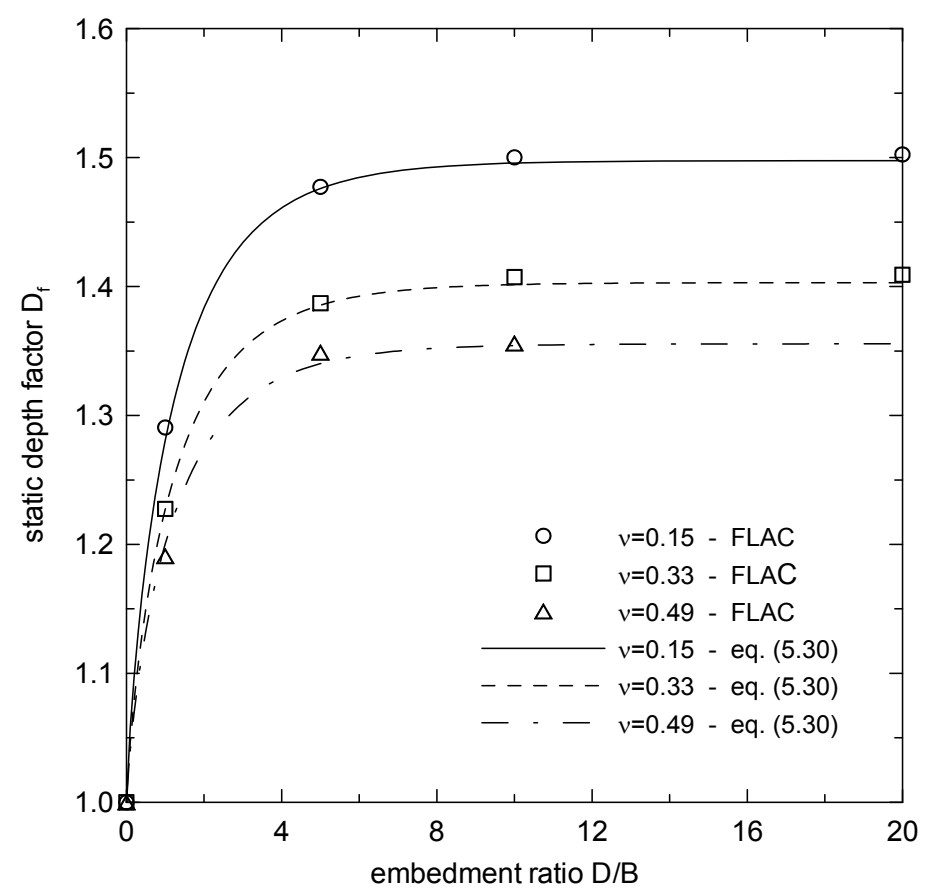

Figure 5.13. Variation of depth factor $D_{f}$ for static footing stiffness with embedment ratio D/B.

The effect of embedment on the dynamic footing stiffness was investigated by Gazetas et al. (1985). However, the embedment $D$ values considered in Gazetas et al. (1985) do not exceed the footing diameter $B$. In pile driving, the embedment ratio $D / B$ values usually lie between 10 and 100 and the motion is highly transient (impact-type response). Moreover, the equations by Gazetas et al. (1985) are developed for steady-state oscillation. Gazetas et al. (1985) and many other researchers suggest that the radiation damping is not affected by the embedment. In the present dynamic analyses, the footing is excited by an initial velocity (impact) applied at all the nodes of the footing, and the ensuing oscillations then dissipate by radiation and hysteretic damping. The soil is assumed to be linear elastic. Rayleigh damping is added in order to simulate soil hysteretic damping. The mass $M$ and the diameter $B$ of the footing were $1.96 \mathrm{t}$ and $0.5 \mathrm{~m}$, respectively. The soil has shear modulus $G$ equal to $100000 \mathrm{kPa}$ and density $\rho$ equal to $1.8 \mathrm{t} / \mathrm{m}^{3}$. The elastic modulus assigned to the footing material was 1000 times greater than that of the soil in order to ensure that the footing will behave as a rigid body. 
Table 5.1. Summary of results from FLAC simulations of embedded vibrating footing.

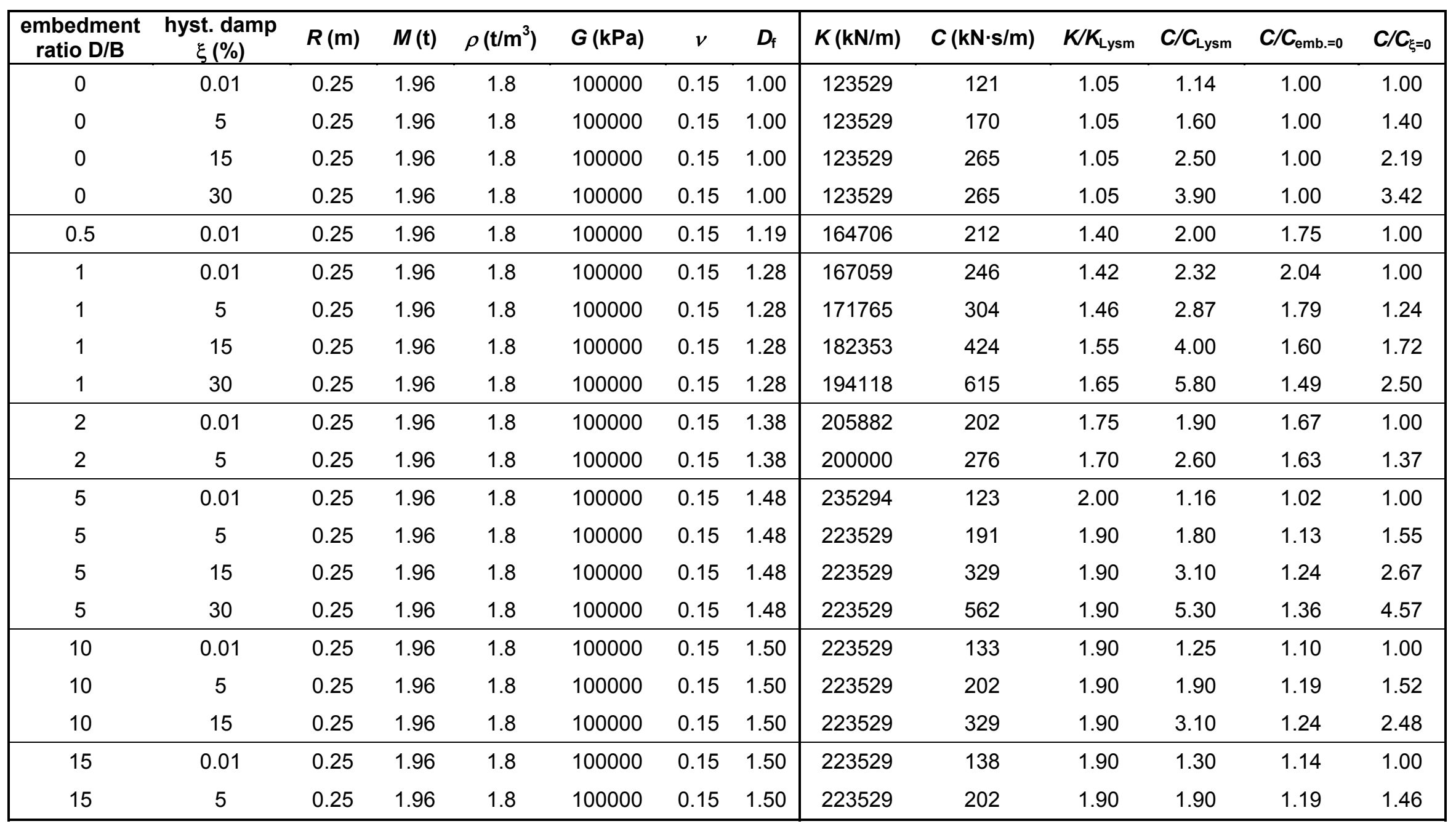


In addition, the footing thickness was set to a small value $(=0.1 \mathrm{~m})$ in order to minimize wave transmission inside the footing mass. The combination of soil stiffness and footing mass is such that the natural period of the footing-system is roughly 0.02 , which is typical of the period of motion of piles during driving. Analyses were performed for $D / B$ from 0 to 15 and hysteretic damping ratio $\xi$ from $0.01 \%$ to $30 \%$. Analyses were performed for a single Poisson's ratio value $(v=0.15)$ because it is expected that the effect of $v$ will be captured by $D_{\mathrm{f}}$ (eq. 5.31). The main output of the analysis was the motion of the mass $M$. Modified values for the spring stiffness $K$ and dashpot coefficient $C$ of Lysmer's analog that account for the embedment effect are determined by matching the footing response observed in each analysis with that predicted by the simple Lysmer's analogue. The dynamic simulation results are summarized in Table 5.1.

The ratio of the observed $K$ values to the corresponding $K_{\mathrm{Lysm}}$ values yielded by eq. (5.29) leads to a depth factor $D_{\mathrm{f}, \mathrm{dyn}}$ for the dynamic base stiffness. From Table 5.1, it can be seen that the ratio $K / K_{\mathrm{Lysm}}$ is larger than the static depth factor $D_{\mathrm{f}}$. Moreover, the hysteretic damping has minimal effect on the stiffness except for $D / B$ equal to 1.0 . The dynamic depth factor can be expressed as

$$
D_{\mathrm{f}, \mathrm{dyn}}=\left(D_{\mathrm{f}}\right)^{1.7}
$$

The stiffness enhancement under dynamic conditions can be attributed to the inertia of the soil above the footing elevation that is dragged down by the soil right underneath the footing. The initial stiffness of the nonlinear spring appearing in eq. (5.27) will be given by

$$
K_{\mathrm{b}, \max }=K_{\text {Lysmer }} D_{\mathrm{f}, \mathrm{dyn}}=K_{\text {Lysmer }}\left(D_{\mathrm{f}}\right)^{1.7}
$$

The ratio $C_{\text {hys }}$ of the observed dashpot coefficient $C$ to the corresponding dashpot coefficient for zero hysteretic damping $C_{\xi=0}$ quantifies the effect of hysteretic damping on the radiation damping. There is a theoretical solution for $c_{\text {hys }}$ for steady-state response based on the correspondence principle of viscoelasticity (Dobry and Gazetas 1986): 


$$
C_{\text {hys }}=1+\frac{2\left(\frac{K_{\mathrm{b}, \max }}{C_{\mathrm{Lysm}} c_{\mathrm{emb}}}\right)}{\omega} \xi
$$

The natural frequency of the pile is

$$
\omega=\mu \frac{\sqrt{E_{\text {pile }} / \rho_{\text {pile }}}}{\mathrm{L}_{\text {pile }}}
$$

where $E_{\text {pile, }}, \rho_{\text {pile }}$ and $L_{\text {pile }}$ are the Young's modulus, the density, and the length of the pile, respectively, and $\mu$ is a coefficient that depends on the boundary conditions at the two ends of the pile. If the pile is assumed to be a rod with one end fixed and the other end free, then $\mu$ is equal to 1.57. If the pile is assumed to have both ends free, then $\mu$ is equal to 3.14 . Since, the conditions at the ends of the pile in reality lie between these two extremes, we will assume $\mu$ equal to 2.4 .

The $\mathrm{C} / C_{\xi=0}$ values in Table 5.1 agree very well with those yielded by eq. (5.34), indicating that eq. (5.34) is also valid for transient motion. The soil mass outside the base failure mechanism is expected to be in the small to intermediate shear strain regime. It is common in the analysis of machine foundations to assume $\xi$ between $2 \%$ and $5 \%$. We use the upper value of $5 \%$ in dynamic pile driving analysis because the amplitude of the quasielastic deformation in the soil outside the bearing capacity mechanism is most likely larger than that occurring under machine foundations.

The ratio of the observed $C$ values to the corresponding $C_{\text {Lysm }}$ values yielded by eq. (5.30) helps us establish a depth factor for the radiation damping. From Table 5.1, we see that $C / C_{\text {Lysm }}$ starts from 1.0 (at $D / B=0$ ) and increases rapidly, attaining a peak value at $D / B=1$. Beyond that point, $C / C_{\text {Lysm }}$ decreases with increasing embedment ratio, reaching a plateau at $D / B=5$ (at the same point $D_{\mathrm{f}}$ reaches its ultimate value). By dividing $C / C_{\text {Lysm }}$ by the corresponding $C_{\text {hys }}$ from eq. (5.34), we obtain values of the depth factor for the radiation damping $c_{\text {emb }}$. The depth factor $c_{\text {emb }}$ values can be fitted closely by the following equation:

$$
C_{e m b}=1.3+\sin \left(1.25\left(\frac{D}{B}\right)-0.35\right) \exp \left[-0.24\left(\frac{D}{B}\right)\right]
$$




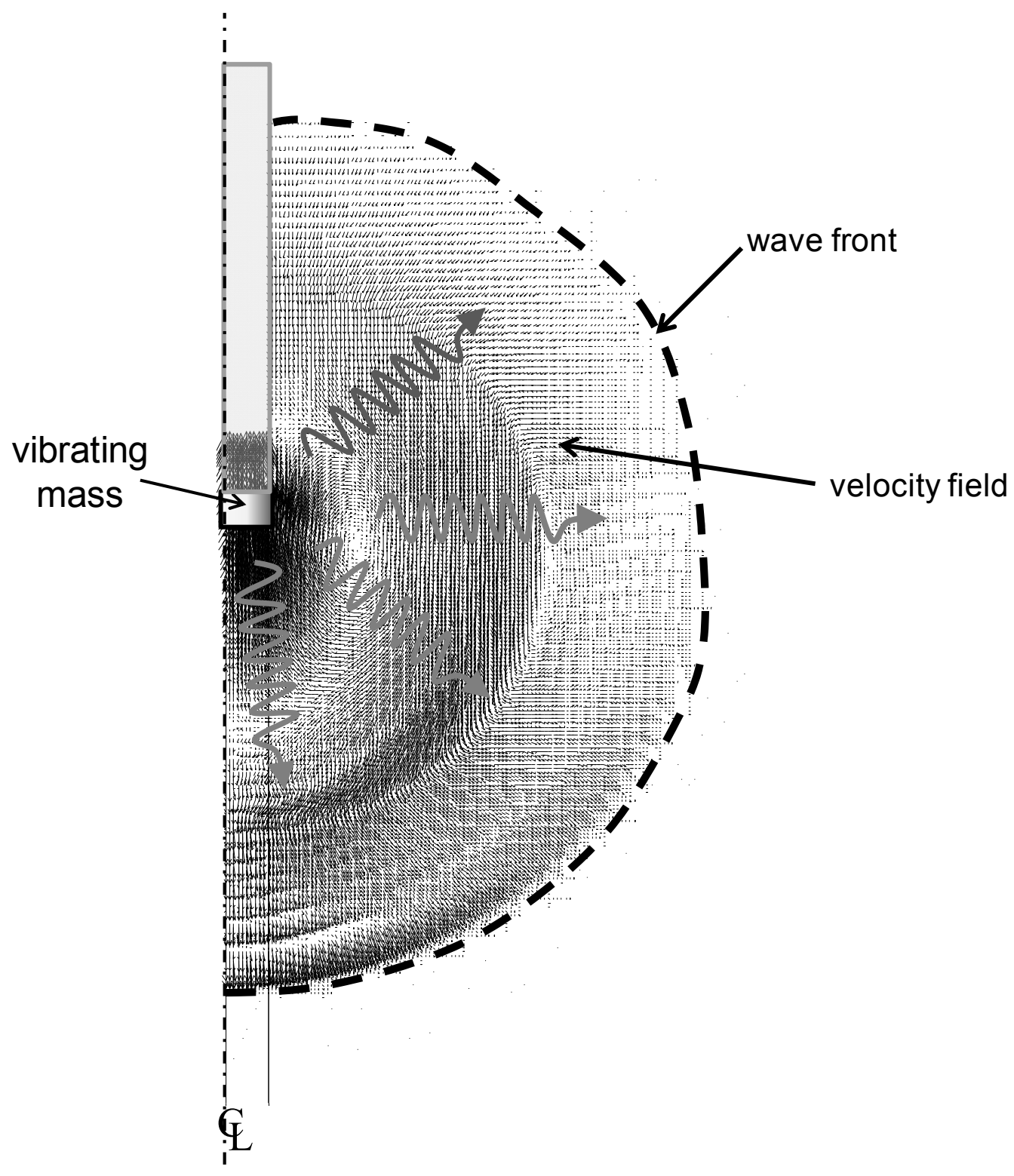

Figure 5.14. Velocity field in dynamic FLAC analysis showing wave propagation in all directions.

The factor $c_{\text {emb }}$ reaches a final value equal to 1.3 for large $D / B$. This means that the radiation damping coefficient for the pile base needs to be $30 \%$ greater than the one for a footing on 
the ground surface. The $30 \%$ increase in $C_{\mathrm{b}}$ is caused by the embedment of the footing into the ground, which allows a certain part of the energy emanating from the vibrating footing to propagate not only downwards and laterally but also upwards. This is easily visualized by plotting the velocity field around the vibrating footing (Fig. 5.14). The wave front increases in size with time in all directions, including the upward direction. Thus, a part of the initial energy is lost in the soil above the pile base elevation.

After the bearing capacity mechanism under the pile base is fully formed, waves stop being transmitted from the pile to the far-field soil, with the possible exception of some minor transmission of energy through the lateral boundaries of the mechanism. The soil in contact with the lateral boundaries of the mechanism provides predominantly horizontal reaction to the rotation of the fan zones of the collapse mechanism. The waves transmitted to the soil mass diminish as the bearing capacity mechanism develops. Hence, the influence of the radiation damping should decrease with increasing plastic deformation inside the mechanism. Radiation damping is associated mostly with the quasi-elastic deformation outside the bearing capacity mechanism since only such deformation will result in wave propagation to the far field. Therefore, the radiation dashpot reaction $R_{\mathrm{b}}{ }^{(\mathrm{D})}$ should be proportional to the rate of the elastic component of the deformation (base settlement):

$$
R_{\mathrm{b}}^{(D)}=\left(C_{\mathrm{Lysm}} C_{\mathrm{emb}} C_{\mathrm{hys}}\right) \dot{\mathrm{w}}_{\mathrm{b}, \mathrm{el}}
$$

Based on eq. (5.27), the elastic component of the pile base settlement is

$$
\dot{w}_{\mathrm{b}, \mathrm{el}}=\frac{1}{\left(1+b_{\mathrm{fb}} \frac{\left|R_{\mathrm{b}}-L I \cdot R_{\mathrm{b}, \mathrm{rev}}\right|}{(L I+1) \times\left|\operatorname{sgn} \cdot R_{\mathrm{bf}}-R_{\mathrm{b}}\right|}\right)^{2}} \dot{w}_{\mathrm{b}}
$$

and the radiation dashpot coefficient for the pile base to be used in eq. (5.26) becomes

$$
C_{\mathrm{b}}=\frac{C_{\mathrm{Lysm}} C_{\mathrm{emb}} C_{\mathrm{hys}}}{\left(1+b_{\mathrm{fb}} \frac{\left|R_{\mathrm{b}}-L I \cdot R_{\mathrm{b}, \mathrm{rev}}\right|}{(L I+1) \times\left|\operatorname{sgn} \cdot R_{\mathrm{bf}}-R_{\mathrm{b}}\right|}\right)^{2}}
$$


At the beginning of the base loading and at the moments of loading-reloading, eq. (5.39) yields a radiation dashpot coefficient value equal to that of the elastic solution. The base radiation dashpot coefficient decreases gradually towards zero as plastic deformation becomes more pronounced and the tangent modulus of the base spring approaches zero.

\subsection{Soil reaction model input parameters}

The input parameters used directly in the shaft and base reaction models are:

1) soil density $\rho$;

2) maximum (i.e., small-strain) shear modulus $G_{\max }$ or, alternatively, the shear wave velocity $V_{\mathrm{s}}$ of the soil;

3) soil's Poisson's ratio $v$;

4) plasticity index $P l$;

5) pile dimensions (radius $R$ and length $L$ );

6) static unit shaft resistance $q_{\mathrm{sL}}$;

7) static unit base resistance $q_{\mathrm{b}}$;

8) soil viscosity parameters $m_{\mathrm{s}}, n_{\mathrm{s}}, m_{\mathrm{b}}$ and $n_{\mathrm{b}}$

Additional soil properties are needed for the indirect estimation of some of the aforementioned parameters, such as $G_{\max }$, the limit resistances, and the viscosity parameters. These are:

a) relative density $D_{\mathrm{R}}$ and the critical state friction angle $\phi_{\mathrm{c}}$ for sandy soils;

b) the undrained shear strength $s_{u}$ and overconsolidation ratio OCR for clayey soils.

It can be seen that most of the input parameters pertaining to the soil are standard parameters that are commonly used in geotechnical engineering. There are no parameters that are specific to the pile driving problem. This reduces significantly the degree of empiricism in the dynamic pile analysis. A number of input parameters are expected to be found in any geotechnical report. These are the soil density $\rho$, the plasticity index $P I$, the relative density $D_{\mathrm{R}}$, and the undrained shear strength $s_{\mathrm{u}}$.

The static unit pile shaft and base resistances can be calculated according to the static design methods presented in Chapter 3 . The $q_{\mathrm{bL}}$ can also be determined directly from CPT data, if available. The unit shaft and base resistances will be inputs to the calculation 
algorithm for drivability analysis. In the case of back-calculation of the static pile capacity from driving data collected during driving or a re-strike test, the $q_{\mathrm{bL}}$ and $q_{\mathrm{sL}}$ will be the output of the analysis procedure. Nonetheless, good initial $q_{\mathrm{bL}}$ and $q_{\mathrm{sL}}$ guesses will accelerate convergence of the calculation process.

\subsubsection{Selection of small-strain soil parameters}

The Poisson's ratio used in the model equations is the small-strain Poisson's ratio. Measuring the Poisson's ratio require elaborate experimental procedures (use of highresolution local strain transducers). Therefore, it is expected that there will be no data regarding the Poisson's ratio. However, Poisson's ratio estimates can be easily made since it shows practically no dependence on soil density and confining stress (Gazetas, 1991). The small-strain Poisson's ratio takes values in the 0.1 to 0.25 range for soils with degree of saturation $S_{\mathrm{r}}<70 \%$, with the lower range values being more representative of sands. Standard values of 0.15 and 0.22 can be used for sandy and clayey soils, respectively, without much impact on the analysis results. The value of Poisson's ratio increases as the degree of saturation increases beyond $70 \%$. For saturated or nearly saturated soils $\left(S_{\mathrm{r}}>95 \%\right)$, the Poisson's ratio should be set equal to 0.5 .

The small-strain shear modulus is an input parameter in both shaft and base reaction models. Direct determination of the small strain shear modulus (or alternatively the shear wave velocity) requires specialized laboratory testing, such as resonant column tests and bender element tests, or field testing, such as cross-hole or down-hole tests. It is highly unlikely that measured $G_{\max }$ or $V_{\mathrm{s}}$ data will be available in routine projects. Such testing is usually done in the context of research or in important projects in seismic regions. Therefore, we need to estimate $G_{\max }$ based on information gathered from the field exploration procedures that are employed in routine projects. These will be the relative density $D_{R}$ of sandy soils calculated from SPT or CPT data and the undrained shear strength $s_{\mathrm{u}}$ for clayey soils determined either from laboratory tests (UC, UU) or CPT data. The plasticity index $\mathrm{PI}$ and the overconsolidation ratio OCR can also be determined from collected specimens.

The small-strain shear modulus of granular soil with rounded particles can be estimated using the correlation established by Hardin and Black (1968): 


$$
G_{\max }=691 \frac{(2.17-e)^{2}}{1+e} \sqrt{p^{\prime} p_{A}}
$$

where $e$ is the void ratio, $p^{\prime}$ is the mean effective stress, and $p_{\mathrm{A}}$ is a reference stress (the standard atmospheric pressure of $100 \mathrm{kPa}=0.1 \mathrm{MPa}=1 \mathrm{kgf} / \mathrm{cm}^{2}=1 \mathrm{tsf}$ ). In the case of granular soil with angular particles, an equation of similar form was also proposed (Hardin and Black 1968):

$$
G_{\max }=323 \frac{(2.97-e)^{2}}{1+e} \sqrt{p^{\prime} p_{A}}
$$

An extended version of eq. (5.41) for clayey/silty soils (Hardin and Drnevich 1972) takes the form

$$
G_{\max }=323 \frac{(2.97-e)^{2}}{1+e}(O C R)^{k} \sqrt{p^{\prime} p_{A}}
$$

The power $k$ to which the overconsolidation ratio $O C R$ is raised is given in Table 5.2. Eq. (5.42) may overpredict the stiffness of silts and clays that have low void ratios, comparable to that of sands (0.4 to 1.0). Kim and Novak (1981) proposed the following equation for clays and silts, which has range of applicability regarding void ratio e from 0.4 to 1.1:

$$
G_{\max }=C_{g} \frac{(2.97-e)^{2}}{1+e}(O C R)^{k} p^{\prime n_{g}} p_{A}^{1-n_{g}}
$$

with $C_{\mathrm{g}}$ in the 54 to 208 range and $n_{\mathrm{g}}$ in the 0.46 to 0.61 range. Equations (5.40) through (5.43) require knowledge of the soil void ratio. In the case of sandy soils, obtaining the void ratio from relative density requires knowledge or good estimation of the minimum and maximum void ratios $\left(e_{\min }\right.$ and $\left.e_{\max }\right)$. 
Table 5.2. Exponent $k$ for eqs. (5.42) and (5.43)

\begin{tabular}{|c|c|}
\hline $\begin{array}{c}\text { Plasticity } \\
\text { Index (PI) }\end{array}$ & $k$ \\
\hline 0 & 0.00 \\
\hline 20 & 0.18 \\
\hline 40 & 0.30 \\
\hline 60 & 0.41 \\
\hline 80 & 0.48 \\
\hline$\geq 100$ & 0.50 \\
\hline
\end{tabular}

There are correlations that give $G_{\max }$ not as a function of the void ratio but as a function of variables that will be readily available in any deep foundation design project, namely the sand relative density $D_{\mathrm{R}}$ and the clay $s_{\mathrm{u}}$. For example, $G_{\max }$ for sands can be estimated from the empirical relationship (Seed and Idriss 1970):

$$
G_{\text {max }}=1000 K_{2, \max } \sqrt{p^{\prime}}
$$

where $K_{2, \max }$ is determined from relative density of the sand (Table 5.3). The mean effective stress in eq. (5.44) and the resulting $G_{\max }$ are in psf.

Table 5.3. Estimation of $K_{2, \max }($ Seed and Idriss 1970)

\begin{tabular}{|c|c|}
\hline $\operatorname{Dr}(\%)$ & $\mathrm{K}_{2, \max }$ \\
\hline 30 & 34 \\
\hline 40 & 40 \\
\hline 45 & 43 \\
\hline 60 & 52 \\
\hline 75 & 59 \\
\hline 90 & 70 \\
\hline
\end{tabular}

For fine-grained soils, $G_{\max }$ can be estimated from $s_{\mathrm{u}}, \mathrm{OCR}$ and PI using the correlation by Weiler (1988):

$$
G_{\max }=K_{\mathrm{u}} \mathrm{s}_{\mathrm{u}}
$$


Table 5.4. Values of $K_{\mathrm{u}}$ after Weiler (1988)

\begin{tabular}{|c|c|c|c|}
\hline \multirow{2}{*}{$P I$} & \multicolumn{3}{|c|}{$K_{\mathrm{u}}$} \\
\cline { 2 - 4 } & OCR=1 & OCR=2 & OCR=5 \\
\hline $15-20$ & 1100 & 900 & 600 \\
\hline $20-25$ & 700 & 600 & 500 \\
\hline $35-45$ & 450 & 380 & 300 \\
\hline
\end{tabular}

Finally, if CPT data is available, one may use the correlations shown in Table 5.5 in order to estimate $G_{\max }$ directly from the measured cone resistance $q_{c}$.

Table 5.5. Correlations between $G_{\max }$ and cone resistance $q_{c}$ (from Kramer 1996)

\begin{tabular}{|c|c|c|c|}
\hline Relationship & Soil Type & Reference & Comments \\
\hline$G_{\max }=1634\left(q_{c}\right)^{0.250}\left(\sigma_{v}^{\prime}\right)^{0.375}$ & $\begin{array}{c}\text { Quartz } \\
\text { Sand }\end{array}$ & $\begin{array}{c}\text { Rix and Stokoe } \\
(1991)\end{array}$ & $\begin{array}{c}\text { Values in kPa; } \\
\text { Based on calibration } \\
\text { chamber and field } \\
\text { tests }\end{array}$ \\
\hline$G_{\max }=406\left(q_{c}\right)^{0.695} e^{-1.130}$ & Clay & $\begin{array}{c}\text { Mayne and Rix } \\
(1993)\end{array}$ & $\begin{array}{c}\text { Values in kPa; } \\
\text { Based on field tests }\end{array}$ \\
\hline
\end{tabular}

\subsubsection{Selection of soil viscosity parameters}

The soil viscosity parameters have been measured in only few research projects. It is practically impossible for site specific measurements of these parameters to be available in routine deep foundation design. Therefore, the parameters $m_{\mathrm{s}}, n_{\mathrm{s}}, m_{\mathrm{b}}$ and $n_{\mathrm{b}}$ will need to be estimated based on the findings of existing research studies. Coyle and Gibson (1970) performed triaxial tests with the soil specimen loaded dynamically by a falling mass. Coyle and Gibson (1970) were the first to propose this power law for the shear strength under dynamic conditions:

$$
R_{\text {dyn }}=R_{\text {stat }}\left(1+m\left(\frac{\dot{w}}{\dot{w}_{\text {ref }}}\right)^{n}\right)
$$


where $\dot{w}_{\text {ref }}$ is a reverence velocity taken as $1 \mathrm{~m} / \mathrm{s}$. The data by Coyle and Gibson (1970) shows that the exponent $n$ is in the 0.18 to 0.26 range for sands and in the 0.11 to 0.25 range for clays. The multiplier $m$ varies from 0.34 to 0.56 for sand and from 0.95 to 1.55 for clays. Dayal and Allen (1975) performed cone penetration tests in sand and clay in the laboratory at various rates of penetration. The penetration rates were always high enough that fully undrained conditions existed in the tests in clays. Results from experiments in clay show that $m_{\mathrm{b}}=0.49$ and $n_{\mathrm{b}}=0.23$ for cone tip resistance, while, for sleeve resistance, $m_{\mathrm{s}}=0.93$ and $n_{\mathrm{s}}=0.34$. Dayal and Allen (1975) observed no gain in cone resistance or sleeve resistance in sands even at penetration velocities up to $0.8 \mathrm{~m} / \mathrm{s}$. Heerema (1979) also did not observe a rate effect on the interface friction between sand and steel. This is in contrast with model pile load tests in sand by Flemming (1958), who observed rate effects that can be modeled using $m_{\mathrm{s}}=0.25$ and $n_{\mathrm{s}}=0.12$. The interface shear tests between clay and steel by Heerema (1979) show that $n_{\mathrm{s}}$ is in the vicinity of 0.2 and $m_{\mathrm{s}}$ ranges from 0.6 to 1.9. Litkouhi and Poskitt (1980) performed model pile load tests in clay, measuring shaft and base resistance independently. According to shaft resistance data, $m_{\mathrm{s}}$ falls between 0.78 and 2.1, with $n_{\mathrm{s}}$ in the 0.16 to 0.57 range. For base resistance, $m_{\mathrm{b}}$ ranges from 0.44 to 1.0 and $n_{\mathrm{b}}$ from 0.17 to 0.37 . Model pile load tests in clay by Brown (2004) yield $m_{\mathrm{s}}$ and $n_{\mathrm{s}}$ values 1.26 and 0.34, respectively, which are in agreement with the Litkouhi and Poskitt (1980) data.

The parameters $n_{\mathrm{s}}$ and $n_{\mathrm{b}}$ fall in a relatively narrow range, with most values being in the vicinity of 0.2 , with only a few cases falling outside the $0.15-0.25$ range. Hence, most researchers proposed to consider a single $n_{\mathrm{s}}=n_{\mathrm{b}}=0.2$ independently of the soil type. For clays, $m_{\mathrm{b}}$ is shown to be less than $m_{\mathrm{s}}$, suggesting that the rate effect is more pronounced for shaft resistance than for base resistance. This can be explained by the fact that the average velocity inside the plastic mechanism below the pile base is less than the base velocity. The $m_{\mathrm{b}}$ and $m_{\mathrm{s}}$ take higher values for clays than for sands. This is because of the high viscosity exhibited by clays compared to sands. The input parameters $m_{\mathrm{b}}$ and $m_{\mathrm{s}}$ are fitting parameters similar to the damping factors $j_{\mathrm{s}}$ and $j_{\mathrm{b}}$ used in the Smith-type reaction models (eqs. 4.5 and 4.8). However, the scatter of the $m_{\mathrm{b}}$ and $m_{\mathrm{s}}$ values is much smaller than that of the $j_{\mathrm{s}}$ and $j_{\mathrm{b}}$ values. This means that in reality the dependence of soil strength on loading rate is highly nonlinear and is better fit by eqs. (5.19) and (5.28) rather than by the Smith-type reaction models, which assume a linear dependence. The observed nonlinearity is captured adequately by the power law proposed by Coyle and Gibson (1970). 
Lee et al. (1988) collected the data of several experimental studies (Coyle and Gibson, 1970; Dayal and Allen, 1975; Dolwin et al., 1979; Heerema, 1979,1981; Litkouhi and Poskitt, 1980) and found that the parameters $m_{\mathrm{s}}$ and $m_{\mathrm{b}}$ correlate relatively well with the shear strength parameters of the soil; the stronger the soil is, the smaller the $m_{\mathrm{s}}$ and $m_{\mathrm{b}}$ values are. Lee et al. (1988) consider a value of 0.2 for both $n_{\mathrm{s}}$ and $n_{\mathrm{b}}$, independently of the soil type. Based on Lee et al. (1988), the following correlations can be established

$$
\begin{aligned}
& m_{\mathrm{s}}=1.65-0.75\left(\frac{s_{\mathrm{u}}}{p_{\mathrm{A}}}\right) \\
& m_{\mathrm{b}}=1.2-0.63\left(\frac{s_{\mathrm{u}}}{p_{\mathrm{A}}}\right)
\end{aligned}
$$

for clays, and

$$
m_{\mathrm{b}}=1.5-0.083\left(\phi-30^{\circ}\right)
$$

for sands, where $\phi$ is the sand peak friction angle in degrees. Lee et al. (1988) assume that the multiplier $m_{\mathrm{s}}$ for the pile shaft in sands is negligible. In this study, we will use the Lee et al. (1988) correlations for clayey soils (eqs. 5.47 nad 5.48). In addition, we will assume $m_{b}=m_{s}=0.3$ for sands in accordance with Coyle and Gibson (1970) and Randolph (2003). The exponents $n_{\mathrm{s}}$ and $n_{\mathrm{b}}$ will be set equal to 0.2 independently of the soil type.

\subsection{Pile driving analysis solution algorithm}

In FEM or FDM, there are two approaches available regarding the discretization of a system's mass: the lumped mass formulation and the consistent mass formulation. According to Cook et al. (2002), a lumped mass formulation is likely to provide better accuracy than a consistent mass formulation, in addition to its reduced computational cost per time step. The pile discretization into springs and masses is shown in Fig. 5.15. The continuous pile is replaced by $\mathrm{N}-1$ lumped masses $\left(M_{\mathrm{i}}\right)$. The ram (pile hammer) is also represented in the system as an additional mass $\left(M_{1}\right)$. The lumped masses are connected to each other through linear springs $\left(K_{\mathrm{i}}\right)$. A force $R_{\mathrm{s}, \mathrm{i}}$ due to the soil around the pile shaft is exerted on each pile lumped mass lying below the ground surface. The base reaction $R_{\mathrm{b}}$ acts on the last $\left(\mathrm{N}^{\text {th }}\right)$ mass. 
The lumped pile masses are given by

$$
M_{\mathrm{i}}=\rho_{\text {pile }} \Delta L_{\mathrm{i}} A
$$

where $\rho_{\text {pile }}$ is the density of the pile material, $A$ is the pile cross-sectional area, and $\Delta L_{i}$ is the length of the pile segment corresponding to the pile lumped mass. The pile segments do not have to have the same length. The pile discretization should generally be consistent with the soil profile at hand. Having pile segments intersecting more than one layer should be avoided, otherwise average soil properties will need to be assigned to the shaft reaction models. The first mass of the system corresponds to the mass of the hammer ram

$$
M_{1}=M_{\mathrm{ram}}=\frac{W_{\mathrm{ram}}}{g}
$$

where $W_{\text {ram }}$ is the weight of the ram of the driving system and $g$ is the acceleration of gravity.

The stiffness of the interconnecting pile springs is given by

$$
K_{\mathrm{i}}=\frac{E_{\text {pile }} A}{0.5\left(\Delta L_{\mathrm{i}}+\Delta L_{\mathrm{i}-1}\right)}
$$

where $E_{\text {pile }}$ is the Young's modulus of the pile. The spring connecting the pile head to the pile hammer needs to combine the stiffness of the first pile element and the stiffness of the ram:

$$
K_{2}=K_{\text {com }}=\frac{\left(\frac{E_{\text {pile }} A}{0.5 \Delta L_{2}}\right)\left(\frac{E_{\text {ram }} A_{\text {ram }}}{L_{\text {ram }}}\right)}{\frac{E_{\text {pile }} A}{0.5 \Delta L_{2}}+\frac{E_{\text {ram }} A_{\text {ram }}}{L_{\text {ram }}}}
$$

where $E_{\mathrm{ram}}, A_{\mathrm{ram}}$ and $L_{\mathrm{ram}}$ are the Young's modulus, the cross-sectional area and the length of the ram. Because tensile forces cannot be transmitted between pile head and ram in the case of ram rebound, the stiffness $K_{\text {com }}$ is set equal to zero whenever the force in the first spring tends to take negative values. 

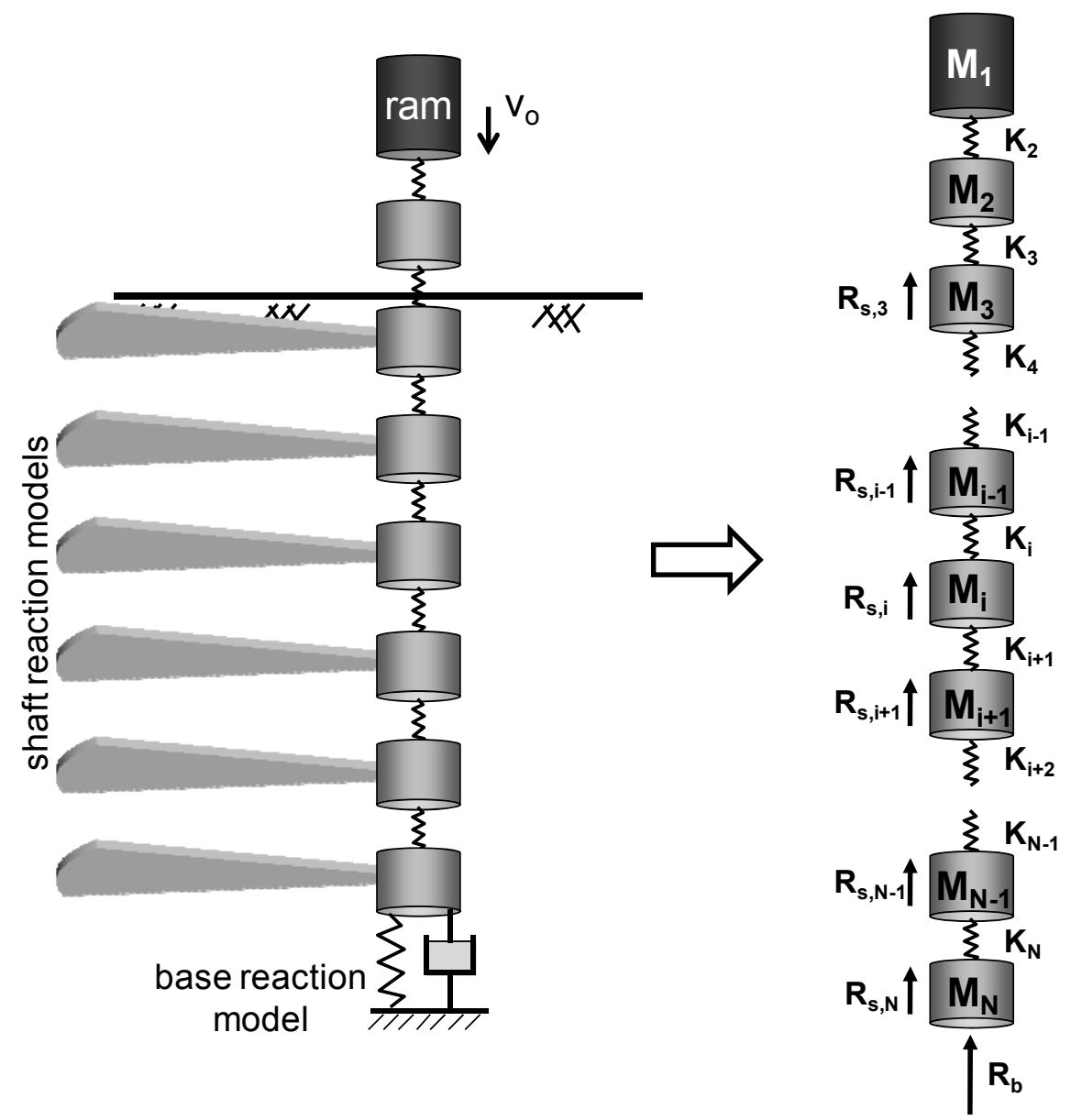

Figure 5.15. Formulation of 1-D dynamic analysis: discretization of pile into lumped masses with soil reactions applied to each lumped mass.

The global stiffness matrix of the system is tridiagonal:

$$
[K]=\left[\begin{array}{ccccccc}
K_{\mathrm{com}} & -K_{\mathrm{com}} & 0 & \cdots & 0 & 0 & 0 \\
-K_{\mathrm{com}} & K_{\mathrm{com}}+K_{3} & -K_{3} & \cdots & 0 & 0 & 0 \\
0 & -K_{3} & K_{3}+K_{4} & \cdots & 0 & 0 & 0 \\
\vdots & \vdots & \vdots & \ddots & \vdots & \vdots & \vdots \\
0 & 0 & 0 & \cdots & K_{\mathrm{N}-2}+K_{\mathrm{N}-1} & -K_{\mathrm{N}-1} & 0 \\
0 & 0 & 0 & \cdots & -K_{\mathrm{N}-1} & K_{\mathrm{N}-1}+K_{\mathrm{N}} & -K_{\mathrm{N}} \\
0 & 0 & 0 & \cdots & 0 & -K_{\mathrm{N}} & K_{\mathrm{N}}
\end{array}\right]
$$

The global mass matrix of any system comprising of lumped masses is a diagonal matrix: 


$$
[M]=0\left[\begin{array}{ccccccc}
M_{\mathrm{ram}} & 0 & 0 & \cdots & 0 & 0 & 0 \\
0 & M_{2} & 0 & \cdots & 0 & 0 & 0 \\
0 & 0 & M_{3} & \cdots & 0 & 0 & 0 \\
\vdots & \vdots & \vdots & \ddots & \vdots & \vdots & \vdots \\
0 & 0 & 0 & \cdots & M_{\mathrm{N}-2} & 0 & 0 \\
0 & 0 & 0 & \cdots & 0 & M_{\mathrm{N}-1} & 0 \\
0 & 0 & 0 & \cdots & 0 & 0 & M_{\mathrm{N}}
\end{array}\right]
$$

Shaft reaction forces $R_{\mathrm{s}, \mathrm{i}}$ are applied to each pile lumped mass that is below the ground surface. The base reaction force $R_{\mathrm{b}}$ is applied at the pile base in addition to the corresponding shaft reaction $R_{\mathrm{s}, \mathrm{N}}$. At each time increment, the values of $R_{\mathrm{s}, \mathrm{i}}$ and $R_{\mathrm{b}}$ are calculated using eq. (5.18b) and eq. (5.26), respectively. In lumped mass systems, the damping matrix is also diagonal. All elements in the damping matrix $[C]$ are equal to zero except the last diagonal element (corresponding to the pile base), which is equal to $C_{b}$. This is because any shaft reaction component due to damping is already included in the force $R_{\mathrm{s}, \mathrm{i}}$

The system of equations of motions is then

$$
[M]\left\{\ddot{w}_{\text {pile }}\right\}+[C]\left\{\dot{w}_{\text {pile }}\right\}+[K]\left\{w_{\text {pile }}\right\}+\{R\}=0
$$

where $\left\{\ddot{w}_{\text {pile }}\right\},\left\{\dot{w}_{\text {pile }}\right\}$, and $\left\{w_{\text {pile }}\right\}$ are the vectors of acceleration, velocity and displacement of the pile lumped masses, and $\{R\}$ is the vector of corresponding soil reactions $\left(R_{\mathrm{s}}\right.$ 's and $\left.R_{\mathrm{b}}\right)$. The system of equations of motions is solved numerically by discretizing the time in small increments $\Delta t$. Any explicit or implicit time integration algorithm can be used. Here we use Newmark's algorithm because it has been found to be more accurate than the central difference algorithm. The displacement, velocity, and acceleration of each pile segment at time $t+\Delta t$ are computed using the following equations, respectively: 


$$
\begin{aligned}
&\{w\}_{(t+\Delta t)}= \frac{1}{\left[K_{\text {eff }}\right]}\left(-\{R\}_{(t)}+[M]\left\{\frac{1}{\beta \Delta t^{2}}\{w\}_{(t)}+\frac{1}{\beta \Delta t}\{\dot{w}\}_{(t)}+\left(\frac{1}{2 \beta}-1\right)\{\ddot{w}\}_{(t)}\right\}+\right. \\
&\left.+[C]\left\{\frac{\gamma}{\beta \Delta t}\{w\}_{(t)}+\left(\frac{\gamma}{\beta}-1\right)\{\dot{w}\}_{(t)}+\Delta t\left(\frac{\gamma}{2 \beta}-1\right)\{\ddot{w}\}_{(t)}\right\}\right) \\
&\{\dot{w}\}_{(t+\Delta t)}=\frac{\gamma}{\beta \Delta t}\left(\{w\}_{(t+\Delta t)}-\{w\}_{(t)}\right)-\left(\frac{\gamma}{\beta}-1\right)\{\dot{w}\}_{(t)}-\Delta t\left(\frac{\gamma}{2 \beta}-1\right)\{\ddot{w}\}_{(t)} \\
&\{\ddot{w}\}_{(t+\Delta t)}=\frac{1}{\beta \Delta t^{2}}\left(\{w\}_{(t+\Delta t)}-\{w\}_{(t)}-\Delta t\{\dot{w}\}_{(t)}\right)-\left(\frac{1}{2 \beta}-1\right)\{\ddot{w}\}_{(t)}
\end{aligned}
$$

where $\left[K_{\text {eff }}\right]$ is the modified stiffness matrix, given by

$$
\left[K_{\text {eff }}\right]=[K]+\frac{\gamma}{\beta \Delta t}[C]+\frac{1}{\beta \Delta t^{2}}[M]
$$

According to Newmark's algorithm, the reaction vector appearing in eq. (5.57) ought to refer to time $t+\Delta t$. In the pile driving problem, the $\{R\}_{(t+\Delta t)}$ cannot be known before calculation of $\{w\}_{(t+\Delta t)}$, which would require performing an iteration for each time increment. Here, the reaction vector corresponds to time $t$ in order to avoid the increased computational cost caused by iterations. It was found that this time lag between input reactions and computed displacement has no effect on the predicted response for the small time increments used in pile driving analysis.

Newmark's algorithm is unconditionally stable as long as the integration factors $\beta$ and $\gamma$ satisfy the inequality

$$
2 \beta \geq \gamma \geq \frac{1}{2}
$$

We use $\beta=0.25$ and $\gamma=0.5$, which correspond to the so-called average acceleration method. Higher $\beta$ and $\gamma$ values introduce into the solution a certain amount of numerical damping, which may be beneficial in cases where spurious high frequency numerical noise arises during integration. These numerical factors control accuracy, numerical stability, and the amount of algorithmic damping. Although the numerical algorithm is unconditionally stable, the time step size needs to be sufficiently small to obtain an accurate solution to the highly 
nonlinear pile driving problem. Generally, the time increment size must be small enough that information does not propagate more than the distance between adjacent nodes during a single time step. This means that the time increment $\Delta t$ needs to be smaller than the threshold value

$$
\Delta t_{\lim }=\frac{\min \left(\Delta L_{\mathrm{i}}\right)}{V_{\mathrm{E}}}
$$

where $V_{E}$ is the wave velocity inside the pile, given by

$$
V_{\mathrm{E}}=\sqrt{\frac{E_{\text {pile }}}{\rho_{\text {pile }}}}
$$

Trial simulations show that results are practically no longer influenced by the time step size for $\Delta t$ values less than $0.1 \Delta t_{\text {min }}$.

The analysis starts with the ram mass impacting the pile head with a velocity $V_{0}$. The impact velocity is given by the equation

$$
\dot{w}_{1}=V_{0}=\sqrt{2 \frac{E_{\mathrm{i}}}{M_{\mathrm{ram}}} \times e_{f}}=\sqrt{2 g h \times e_{f}}
$$

where $g$ is the acceleration of gravity, $h$ is the hammer drop height, and $e_{\mathrm{f}}$ is the driving system efficiency expressing the energy losses inside the pile hammer system and inside the assembly placed between pile head and hammer (pile helmet, hammer and pile cushions). $E_{\mathrm{i}}$ is the initial potential energy of the hammer $(M \times g \times h$; also called the 'theoretical' energy). In the case of diesel hammers, the boundary condition at the pile head is more accurately represented by a hammer force time history at the pile head (force pulse) instead of a ram mass with initial velocity. For simplicity, we will not account explicitly for the presence of pile helmet, anvil and cushion. Their effect can be implicitly incorporated in the analysis by altering accordingly the ram mass (or the pile lumped mass) and stiffness of the first spring. Any energy losses in the assembly placed between pile head and hammer can be accounted for through the efficiency $e_{f}$. 



\section{CHAPTER 6. FIELD AND MODEL PILE LOAD TESTS}

This chapter presents the results from the experiments done on two full-scale steel pipe piles and six model pipe piles. The experiments include 1) pile driving monitoring and static load testing of the full-scale pile, 2) pile driving monitoring and static load testing of the model piles. Procedures followed in conducting the pile tests are presented in detail. Pictures taken during the preparation of the pile and during the field tests are shown in the APPENDIX. The results are used in Chapter 7 for validating the proposed dynamic pile analysis modeling approach discussed in Chapter 5 .

\subsection{Field pile load test in Jasper County}

A full-scale steel pipe pile was installed on the north side of a bridge construction project over Oliver ditch, on State Road 49, in Jasper County, Indiana. The purpose of this field load test was to examine setup effects in clay as well as to study the dynamic behavior of piles during driving. Figure 6.1 shows the location of all piles installed at the site as well as the location of instrumentation and in situ testing (SPT and CPT locations marked with 'S' and ' $C$ ', respectively). There were two instrumented test piles; one was a closed-ended steel pipe pile (MP1) and the other an H-pile (MP2). Nine other piles acted as reaction piles for the static load tests. In the present report we will focus on the results obtained from the closed-ended pipe pile.

\subsubsection{Laboratory and in situ testing}

Extensive in situ and laboratory testing was performed to characterize the soil profile and obtain the soil properties for each layer in the profile. The split spoon samples obtained from different depths showed that the soil profile consisted mainly of a thick deposit of clayey silt and silty clay down to $25 \mathrm{~m}$ depth. Several seams of sand and silt were also present. Table 6.1 summarizes the soil property values determined by laboratory testing. Figure 6.2 shows data from CPT logging at three locations close to pile MP1. It can be seen that the soil profile consists of soft to moderately stiff clay and silt and medium dense to dense clayey 
sand down to a depth of $17 \mathrm{~m}$, at which point a $2 \mathrm{~m}$-thick layer of very stiff silt is encountered $\left(q_{\mathrm{c}}\right.$ up to 50MPa).

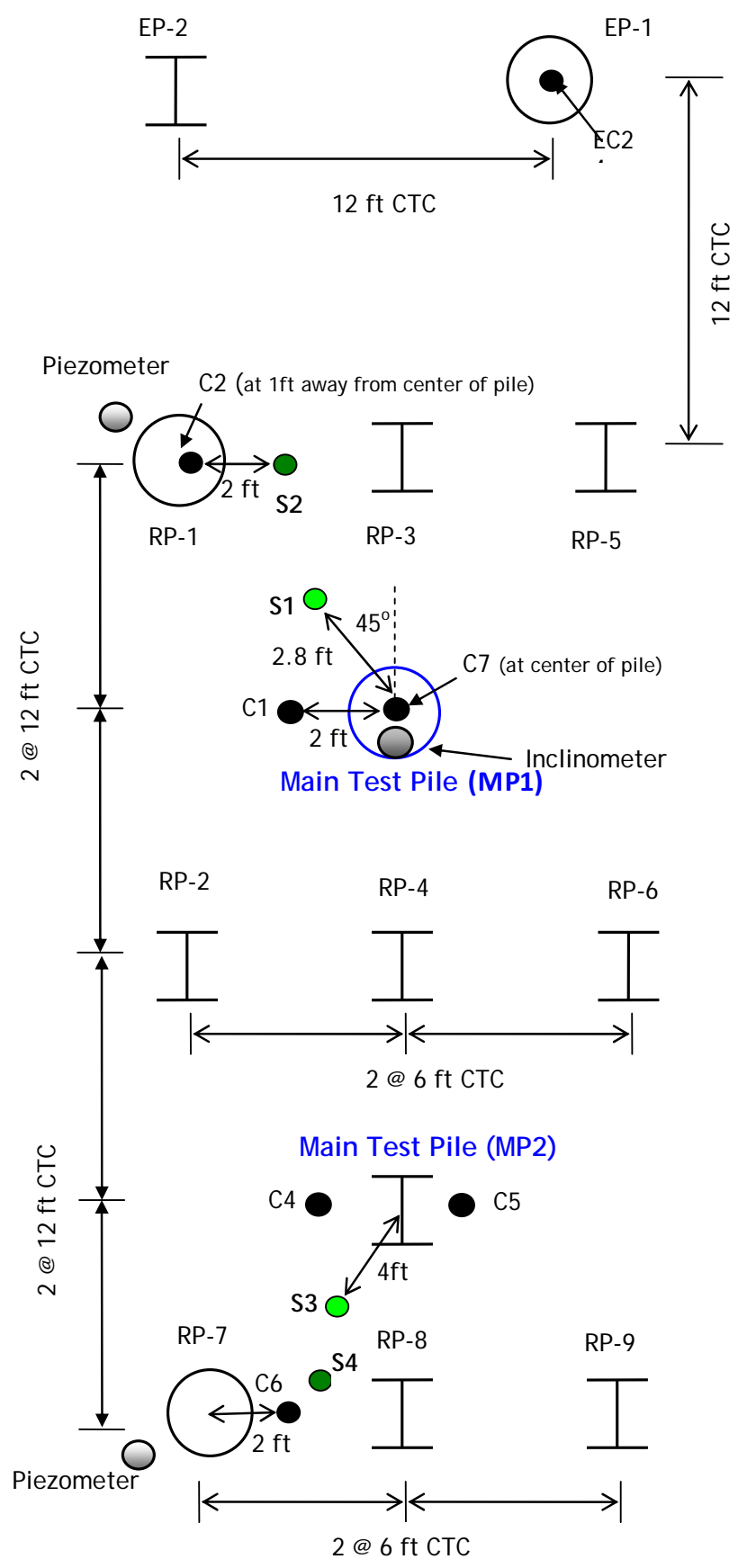

Figure 6.1. Plan view of field pile test layout 
Comparison between the data from CPT loggings $\mathrm{C} 2$ and $\mathrm{C} 7$, which are $5 \mathrm{~m}$ apart, reveals that the variability of the soil profile in the horizontal direction is very small. Cone penetration resistance is compared with SPT blowcounts from nearby boreholes in Figure 6.3. All the samples recovered were found to be fully saturated (degree of saturation very close to $100 \%)$. The water table was estimated to be at $1 \mathrm{~m}$ depth at the time of testing.

Four vibrating wire piezometers were also installed to monitor the excess pore pressure generated in the soil during pile driving. The piezometers were installed close to the boundaries of the test site and at a safe distance from the piles (Fig. 6.1) in order to avoid being damaged during pile driving. At each location, two piezometers were installed at two different depths $(13.5 \mathrm{~m}$ and $17.8 \mathrm{~m})$ inside the clay layers. A Campbell Scientific datalogger (model CR5000) was used to record the piezometer signals. The datalogger has the capabilities of recording under both static (long-term) and dynamic conditions (very-short term).

Table 6.1. Soil properties for samples obtained from Jasper County pile load test site

\begin{tabular}{|c|c|c|c|c|c|c|c|}
\hline Soil Type & $\begin{array}{c}\text { Depth } \\
(\mathrm{m})\end{array}$ & $\begin{array}{c}\mathrm{PI} \\
(\%)\end{array}$ & $\begin{array}{c}\text { Total Unit } \\
\text { Weight } \\
\left(\mathrm{kN} / \mathrm{m}^{3}\right)\end{array}$ & $\mathrm{D}_{\mathrm{R}}(\%)$ & $e_{0}$ & OCR & $\begin{array}{c}s_{u} \\
(\mathrm{kPa})\end{array}$ \\
\hline Organic & $0-1$ & 89 & 13.4 & - & 3.56 & - & - \\
\hline Silty Sand & $1-4$ & 0 & $20.0^{*}$ & 78 & - & - & - \\
\hline Clayey Sand & $4-7$ & 0 & $20.0^{*}$ & 52 & - & - & - \\
\hline Sandy Clay & $7-8$ & 0 & $20.0^{*}$ & - & - & - & - \\
\hline Clayey Sand & $8-9$ & 0 & $20.0^{*}$ & 81 & - & - & - \\
\hline Silty Clay & $9-10$ & 19 & 20.1 & - & 0.73 & 3.2 & 220 \\
\hline Clayey Silt & $10-12$ & 10 & 20.6 & - & 0.63 & 1.9 & 320 \\
\hline Silty Clay & $12-14$ & 9 & 21.9 & - & 0.45 & 4.9 & 103 \\
\hline Clayey Silt & $14-16$ & 10 & 21.6 & - & 0.4 & 2 & 292 \\
\hline Clayey Silt & $16-17$ & 10 & 21.6 & - & 0.4 & 2 & 292 \\
\hline Stiff Silt & $17-18.4$ & 0 & $22.0^{*}$ & $95-100$ & - & - & - \\
\hline
\end{tabular}

* These values are assumed. It was not possible to obtain laboratory test data because of high sample disturbance. 

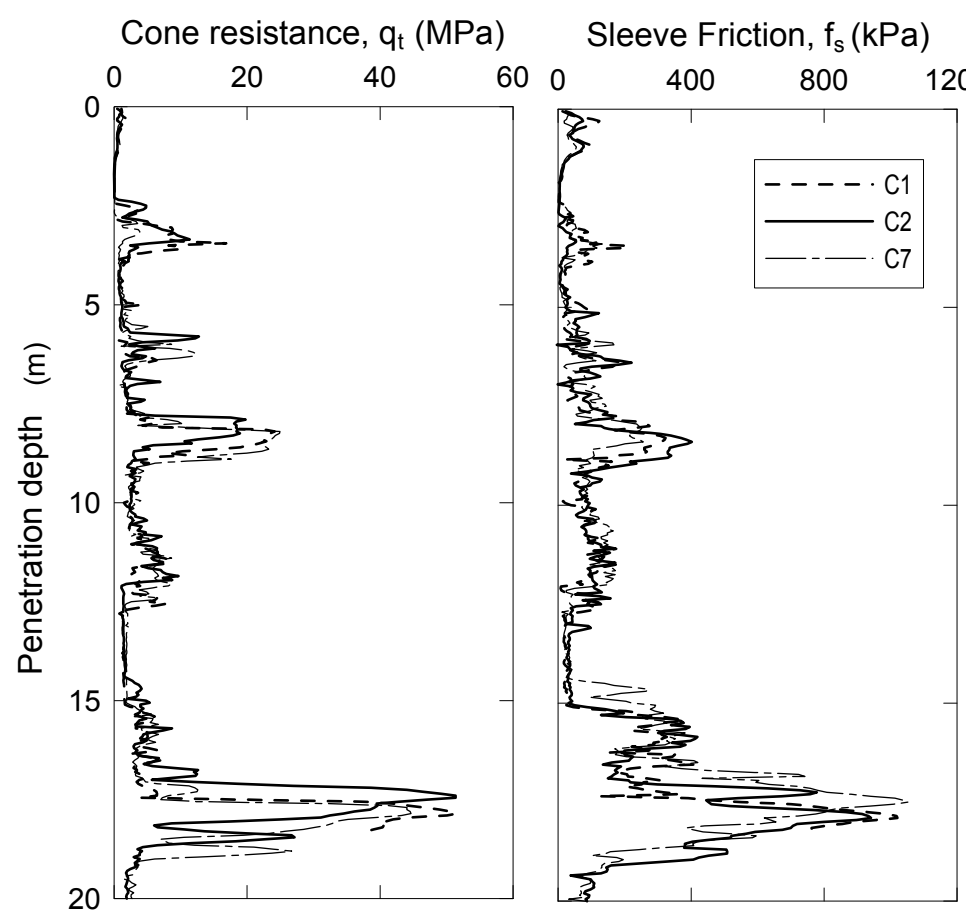

Pore Pressure $(\mathrm{kPa})$

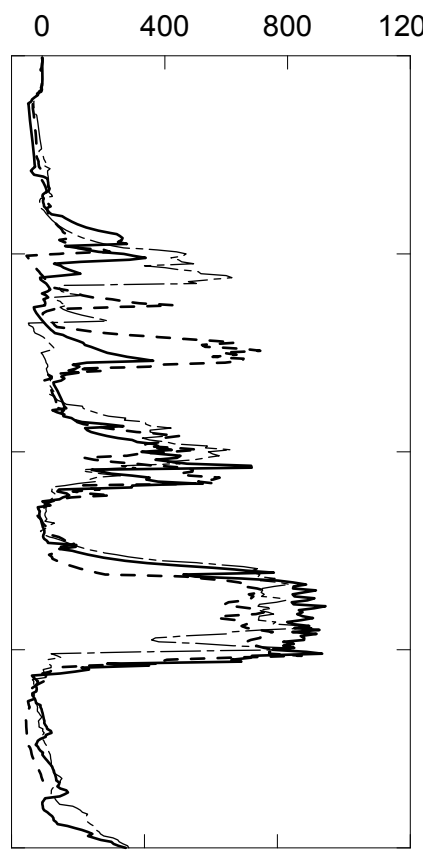

Figure 6.2. Cone penetration test results

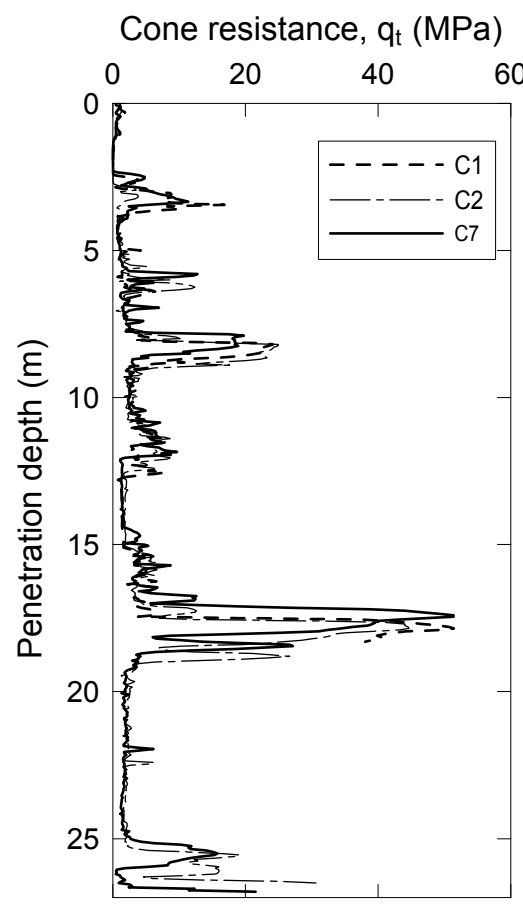

$$
\text { SPT N-value (enlarged) }
$$

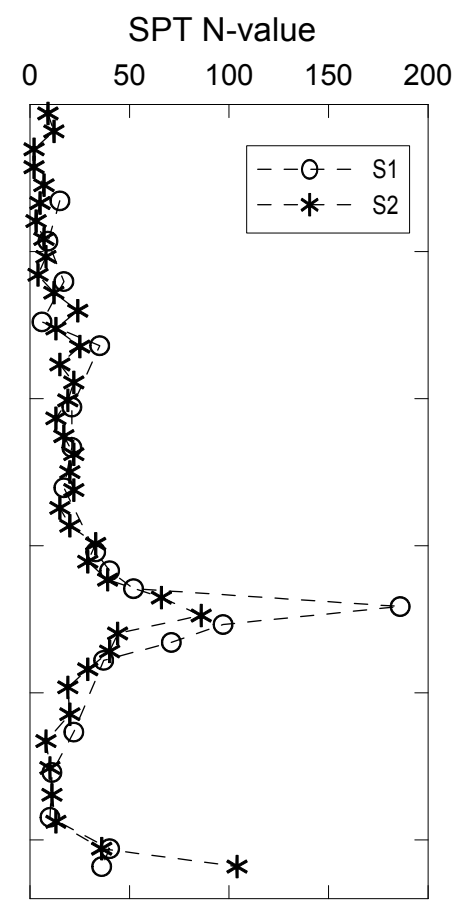

Figure 6.3. Comparison between CPT and SPT test results 


\subsubsection{Pile Instrumentation}

The closed-ended pipe pile was a spiral weld steel pipe with an outer diameter of $356 \mathrm{~mm}$ (14 in) and a wall thickness of $12.7 \mathrm{~mm}(0.5 \mathrm{in})$. The end was closed with a $25.4 \mathrm{~mm}$ (1in) thick steel plate welded to the base. The pile was embedded approximately $0.3-0.4 \mathrm{~m}$ in the very stiff clay layer to a depth of $17.4 \mathrm{~m}$ below ground level. The total length of the pile was about $20 \mathrm{~m}$, including the part above ground. The instrumentation of the pile included strain gages, accelerometers, and strain transducers. Strain gages were used in the static load tests to determine the static shaft and base resistance of the pile. Accelerometers and strain transducers (PDA instrumentation) were used to obtain the pile acceleration and force time histories at the pile head and at the pile base.

Thirty four (34) Geokon vibrating-wire strain gages (model 4150) were attached to the closed-ended pipe pile at 17 levels. Two gages were attached diametrically opposite at each elevation. These strain gages measure the strain induced by loading as the length of the vibrating wire changes. This type of strain gage is only suitable for static measurements because it cannot measure the high-frequency changes in strain present in dynamics testing. The data acquisition system for the vibrating wire strain gages was the Micro-10 Geokon datalogger (model 8020). Three multiplexers (model 8032-16-1) were used to allow the datalogger to capture the signal from all gages simultaneously. The strain gages were carefully placed away from the welding joints of the spiral weld and the pile surface was smoothed and cleaned before the strain gages were welded to their location. In order to protect the gages from the wet conditions expected on site, each gage was individually covered with a steel cap and sealed with silicone. An iron angle, $76 \mathrm{~mm}$ (3in) wide and $6 \mathrm{~mm}$ (0.24in) thick, was also used to protect all the strain gages and their cables from direct contact with the soil during pile driving. The locations of all strain gages are shown in Fig. 6.4. Pictures taken during the preparation of the pile and during the field tests are shown in the APPENDIX. The stress at the pile shaft is calculated from the measured strain based on the Young's modulus of the pile material using Hooke's law. The base resistance was calculated from the measurements obtained from the bottom row of strain gages. The total pile resistance was also measured by a load cell placed between the hydraulic jack and the reaction beam. There is a good match between the total pile resistance calculated based on the strain gage measurements and that measured by the load cell.

For the dynamic testing, two strain transducers and two piezo-electric accelerometers were attached to the pile shaft two diameters below the pile head. A second set of strain transducers (F1 and F2) and piezo-electric accelerometers (A1 and A2) were 
attached to the pile base as shown in Fig. 6.5. The pile driving analyzer (PDA) was used to record the acceleration and force data during pile driving.

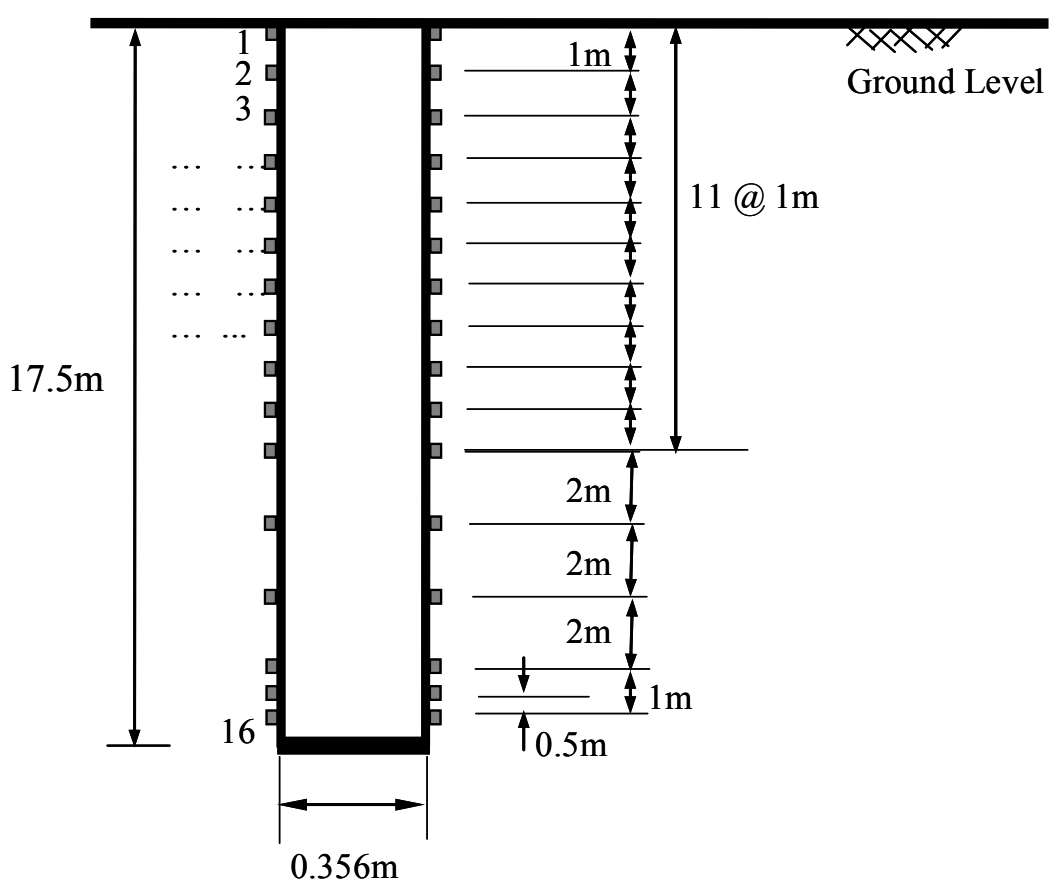

Figure 6.4. Location of strain gages on pipe pile

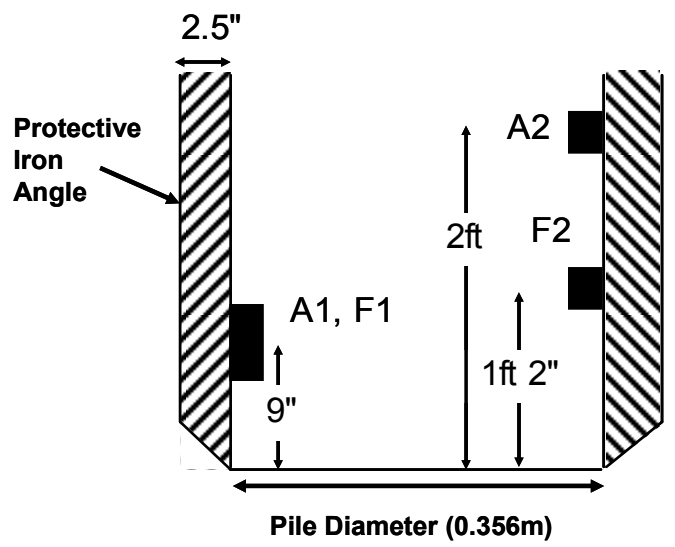

Figure 6.5. Location of accelerometers $\mathrm{A} 1$ and $\mathrm{A} 2$ and strain transducers $\mathrm{F} 1$ and $\mathrm{F} 2$ at pile base 


\subsubsection{Pile driving and dynamic test results}

The ICE-42S single-acting diesel hammer was used to drive all piles in this project. The ram weight was $18.2 \mathrm{kN}$. The maximum hammer stroke is $3.12 \mathrm{~m}$ and the rated maximum driving energy was $56.8 \mathrm{~kJ}$. The actual driving energy delivered to the pile head was on average about $36 \%$ of the initial potential (rated) energy of the ram. From 0 to $10 \mathrm{~m}$ of pile penetration, the observed hammer stroke was about $2 \mathrm{~m}$ on average. By the time the pile had reached the final penetration depth of $17.4 \mathrm{~m}$, the observed stroke had risen to the value of $2.8 \mathrm{~m}$.

Fig 6.6 shows the cumulative hammer blow counts and the pile penetration per blow vs. depth of penetration. Driving resistance is negligible for the first $3 \mathrm{~m}$ of depth, as the pile penetrates the superficial layers of organic soil and soft clay. The blow count increased gradually with depth until the stiff silt layer (bearing layer) was reached. At approximately $17 \mathrm{~m}$ depth, there was a large increase in the number of hammer blows as the pile penetrated the stiff silt layer $(20 \mathrm{~mm} / \mathrm{blow})$. Driving stopped when the pile was sufficiently embedded in that layer. The pile was driven in two sections because the total pile length was greater than the length that the hammer guide could accommodate.

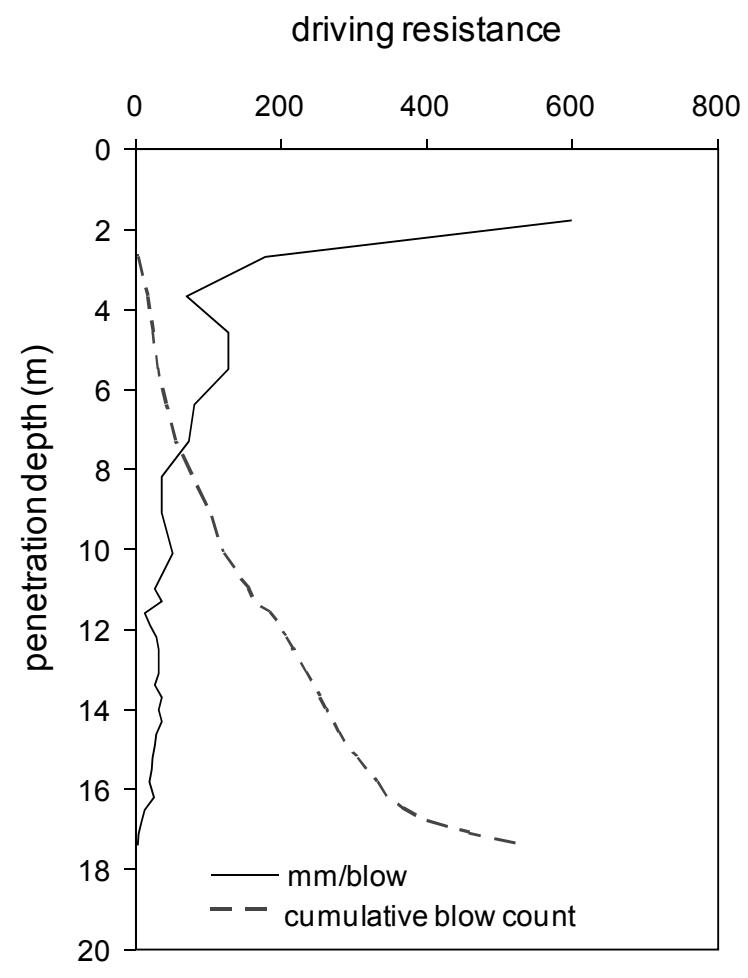

Figure 6.6. Driving resistance of closed-ended pipe pile 
The acceleration and force time histories were recorded for every hammer blow using the Pile Driving Analyzer (PDA). The velocity and displacement time histories are extracted from the acceleration records through numerical integration with time. This is done automatically by the PDA. Figures 6.7 and 6.8 show examples of the velocity and displacement time histories at the pile head, respectively. The peak velocity at the pile head is of the order of $6 \mathrm{~m} / \mathrm{sec}$, and the total duration of the motion is $0.075 \mathrm{~s}$ to $0.2 \mathrm{~s}$. The pile head displacement increases with time until a peak is reached, after which it decreases to a final residual displacement (the pile "set"). The observed shape of the displacement vs. time curves is typical of the pile response during driving. Both peak and residual pile head displacement decrease as the pile penetrates deeper into the ground.

Acceleration records were obtained also from accelerometers placed close to the pile base. However, the acceleration records obtained at the pile base were found to be unreliable. Fig. 6.9 shows the displacement time histories at the pile base for various hammer blows. The final displacement values at the pile base are found to be negative, while the corresponding pile set was positive. The pile base motion time histories are erroneous probably due to excessive noise caused by reflections and wave scattering at the pile base. The protective iron angle and the base plate with its welding seams constitute irregularities of the pile cross-section. The excessive noise renders the numerical integration with time unreliable. It is possible that the accelerometer calibration and configuration was unsuitable for the frequency content of the motion it was meant to record. It is also possible that the poor-performance of the accelerometers was due to malfunctioning. Finally, Fig. 6.10 shows the force histories at the pile base derived from the strain transducer measurements. 


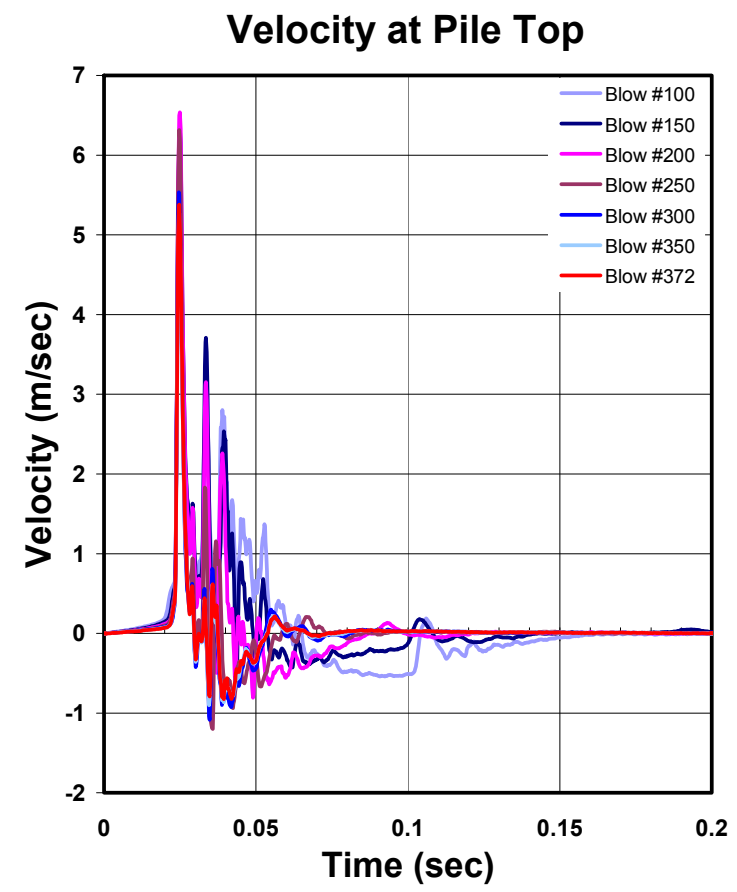

Figure 6.7. Velocity records at pile top for different hammer blows (pile MP1, Jasper County).

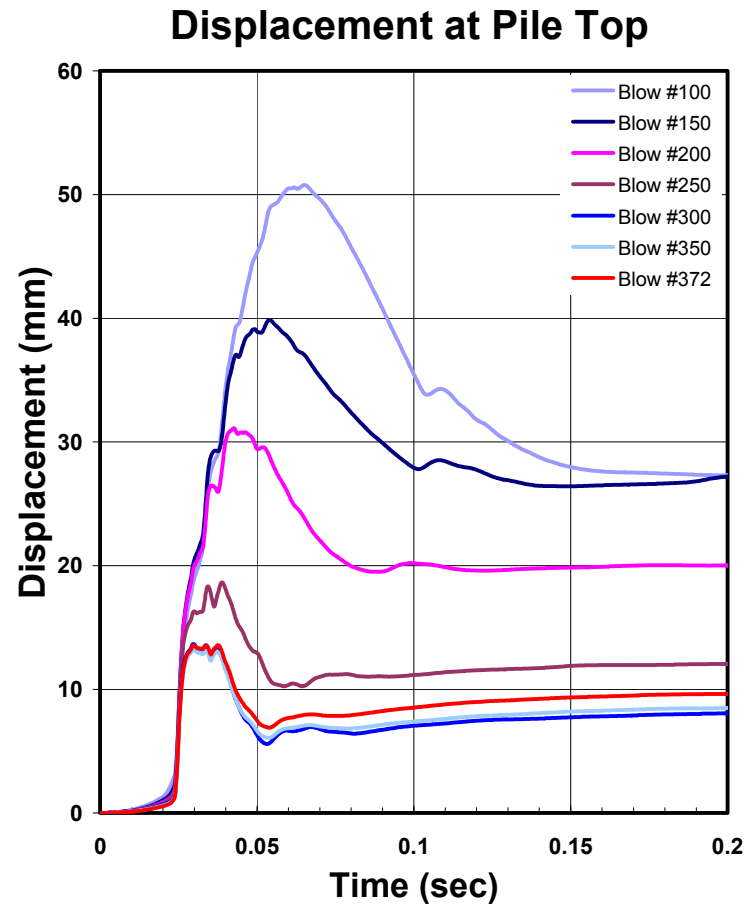

Figure 6.8. Displacement records at pile top during different driving stages of pile MP1 in Jasper County 
Pile Base Displacement

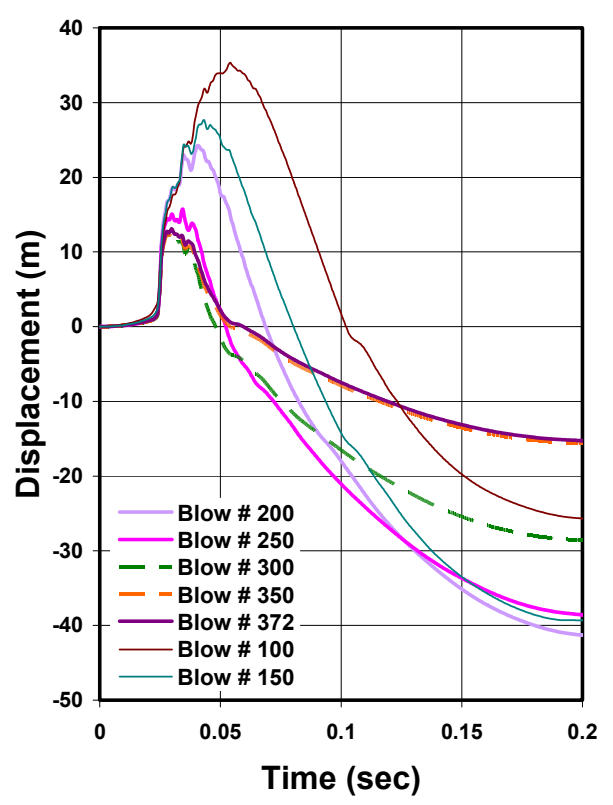

Figure 6.9. Displacement time histories at pile base for different hammer blows (pile MP1, Jasper County).

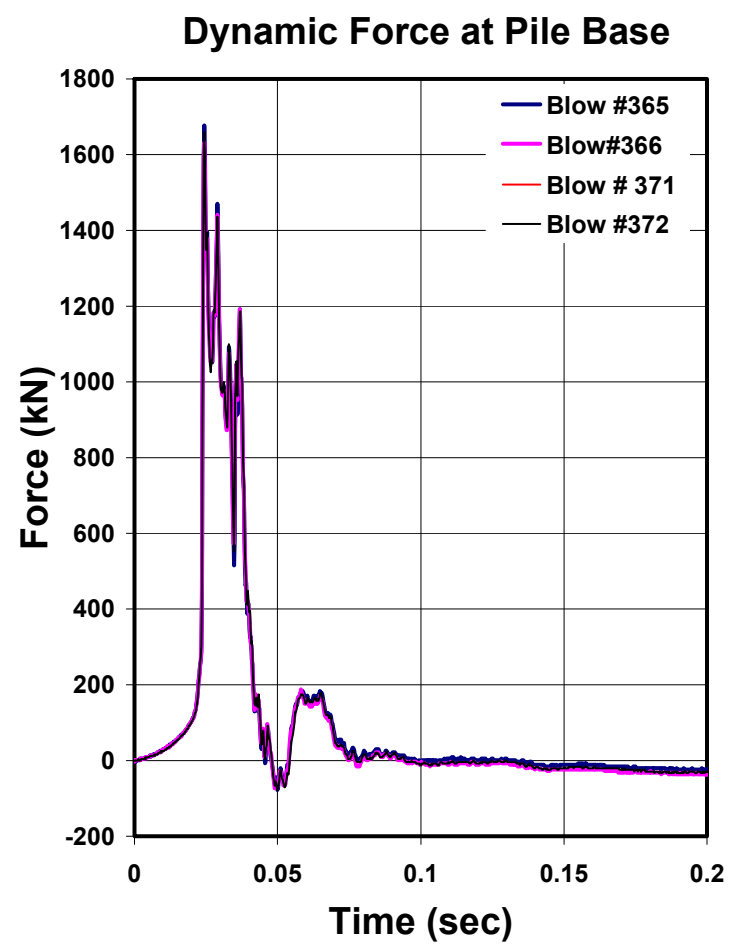

Figure 6.10. Force records at pile base during final driving stages (pile MP1, Jasper County). 


\subsubsection{Static Pile Load Testing}

The reaction frame that was designed for the static load test is shown in Fig. 6.11. The reaction piles were driven to depths of either $17.4 \mathrm{~m}$ or $24.5 \mathrm{~m}$, depending on the required resistance during the static load tests. Two static load tests were conducted on the closedended pipe pile. The total load applied to the pile head was measured by a load cell with a capacity of 2.0 MN. The vertical settlement of the pile head was measured by two dial gages attached to two reference beams. The values of all strain gages were zeroed before the start of each static load test. The load is applied in increments on the pile head using a hydraulic jack. At each loading increment, the settlement is recorded after 5, 15, 35, 55, 75, 95, and $120 \mathrm{~min}$. The next loading increment is applied when the pile settlement stabilizes (settlement rate becomes less than $0.025 \mathrm{~mm} / \mathrm{h}$ ). The magnitude of the loading increments at the early stages of each test was initially $0.4 \mathrm{MN}$ and was reduced progressively to 0.05 $\mathrm{MN}$ near the end of the test. Decreasing the loading increment as the pile gets closer to failure allows accurate determination of the plunging load.

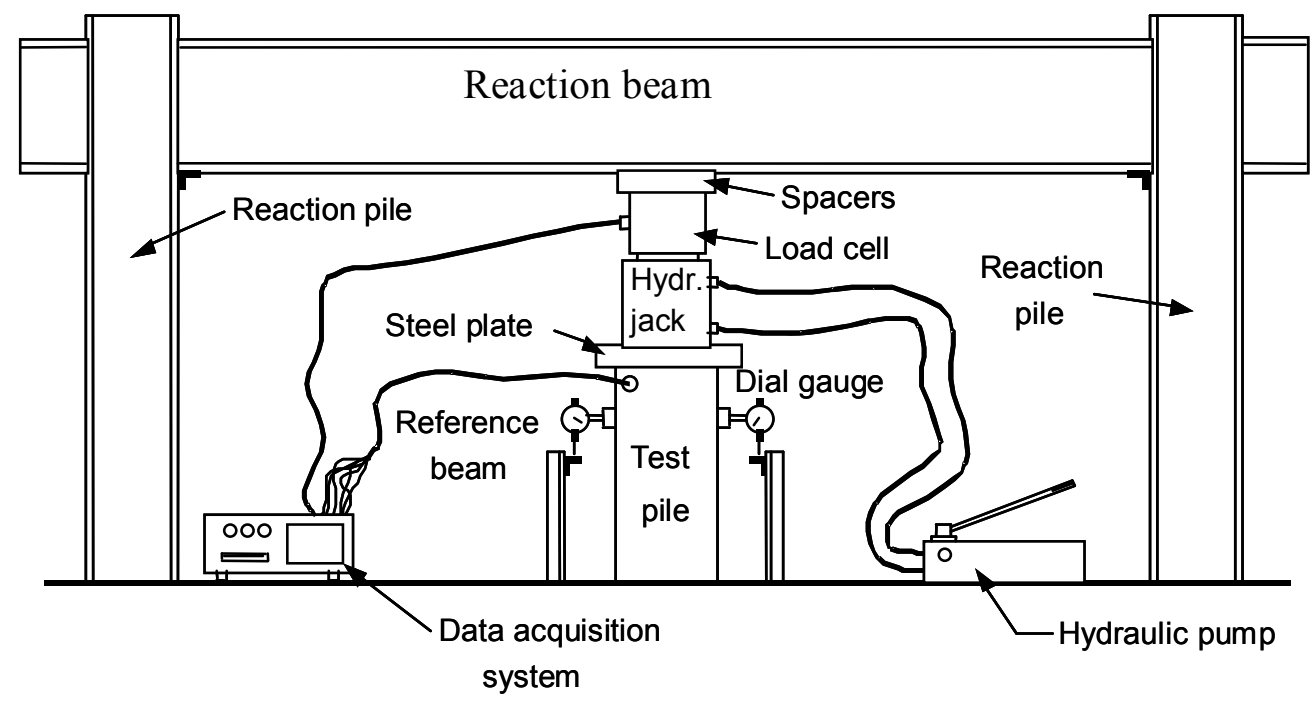

Figure 6.11. Reaction frame system and setup for static load test

The two static load tests were carried out 50 (test No.1) and 90 (test No.2) days after driving, respectively. Therefore, it is expected that the pile load test results will reflect some amount of pile setup. Pile setup is the phenomenon of increase in pile capacity with time 
and is particularly pronounced in the case of clayey soils because of the development of pore pressure during driving, which then dissipates slowly after pile installation. Fig. 6.12 shows the axial load-settlement curves from both static load tests. Both curves approach the same limit load of $1900 \mathrm{kN}$, although the second test shows a much stiffer response. CAPWAP analysis based on the PDA-recorded force and velocity at the pile head during driving yielded a limit load of $908 \mathrm{kN}$. Part of the difference between pile load test and CAPWAP results should be attributed to pile setup.

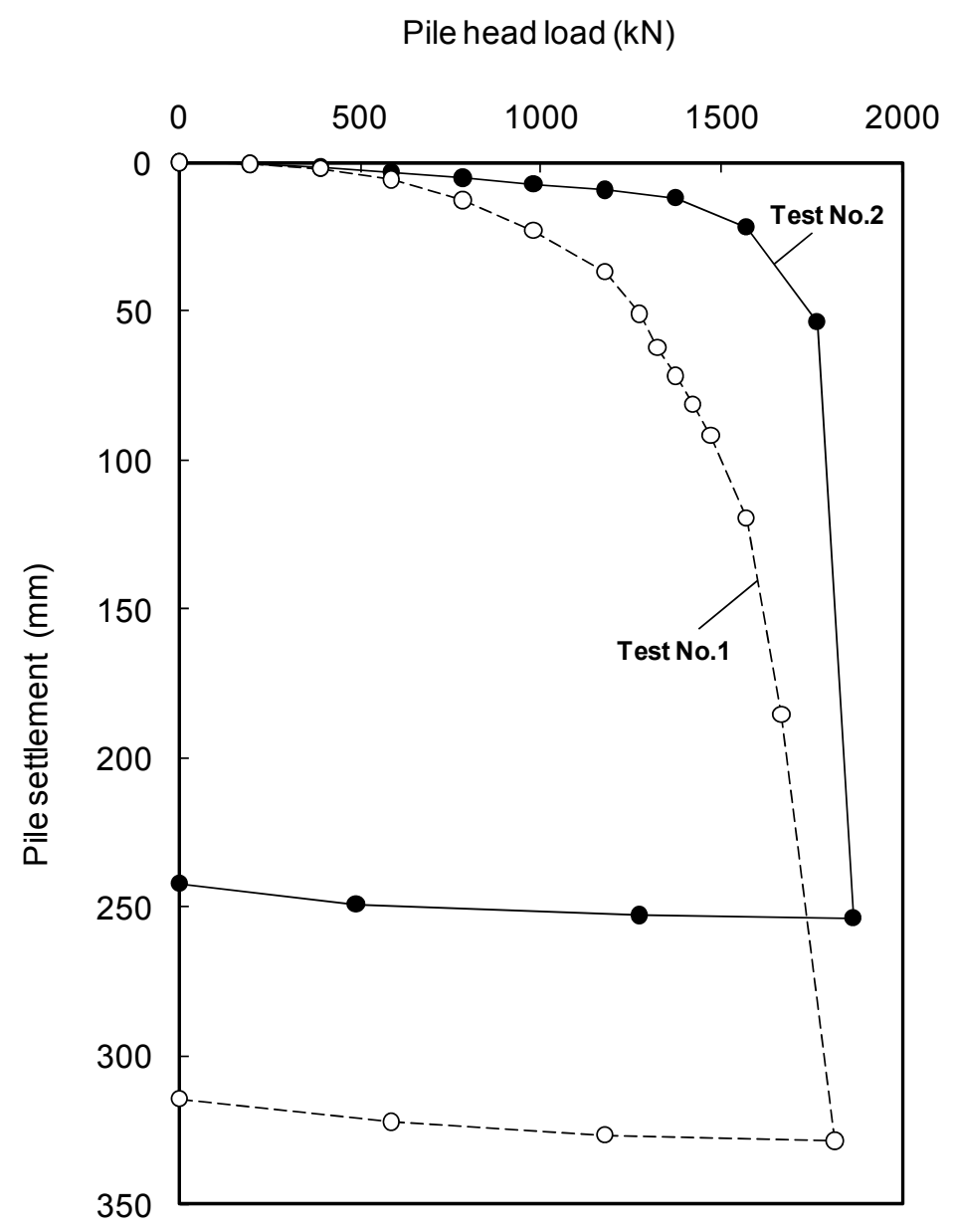

Figure 6.12. Load-settlement curves for field static load tests on pile MP1 in Jasper County 


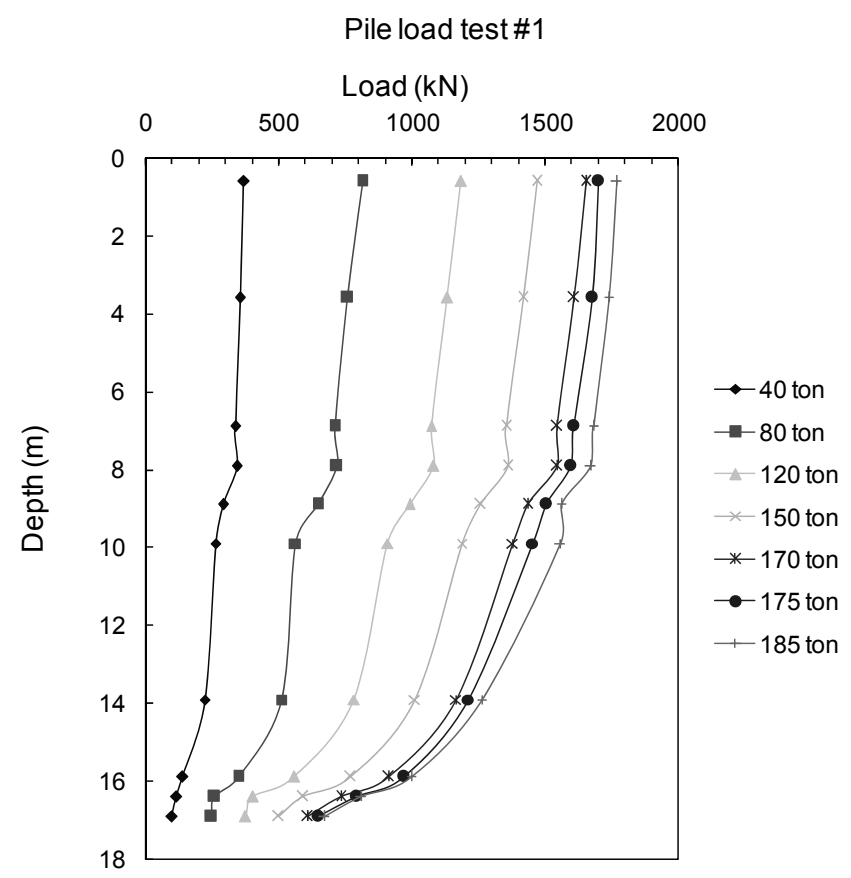

(a)

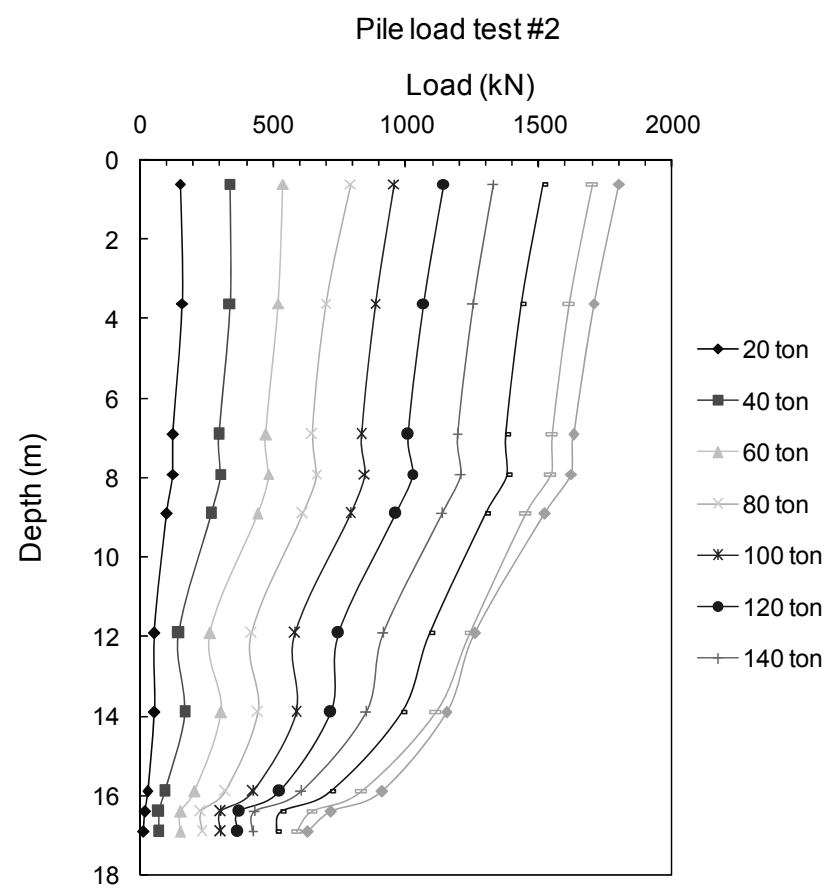

(b)

Figure 6.13. Load transfer curves from the static load tests on pile MP1 in Jasper County. 
The load transfer curves derived from the strain gage data shown in Fig. 6.13. The load transfer curves stop changing inclination and shape very early in the loading process, at a fraction of the pile limit load. This because the limit shaft resistance is reached well before the final loading, while the base load keeps increasing. After limit shaft capacity is reached, each load transfer curve is simply a parallel translation of the previous one. Fig. 6.14 shows the unit shaft resistance derived from strain gage data. The limit shaft resistance is very small in the upper $8 \mathrm{~m}$ of the soil profile, at which point it starts increasing with depth. This is consistent with the CAPWAP results, which show almost zero limit shaft resistance above $8 m$ depth.

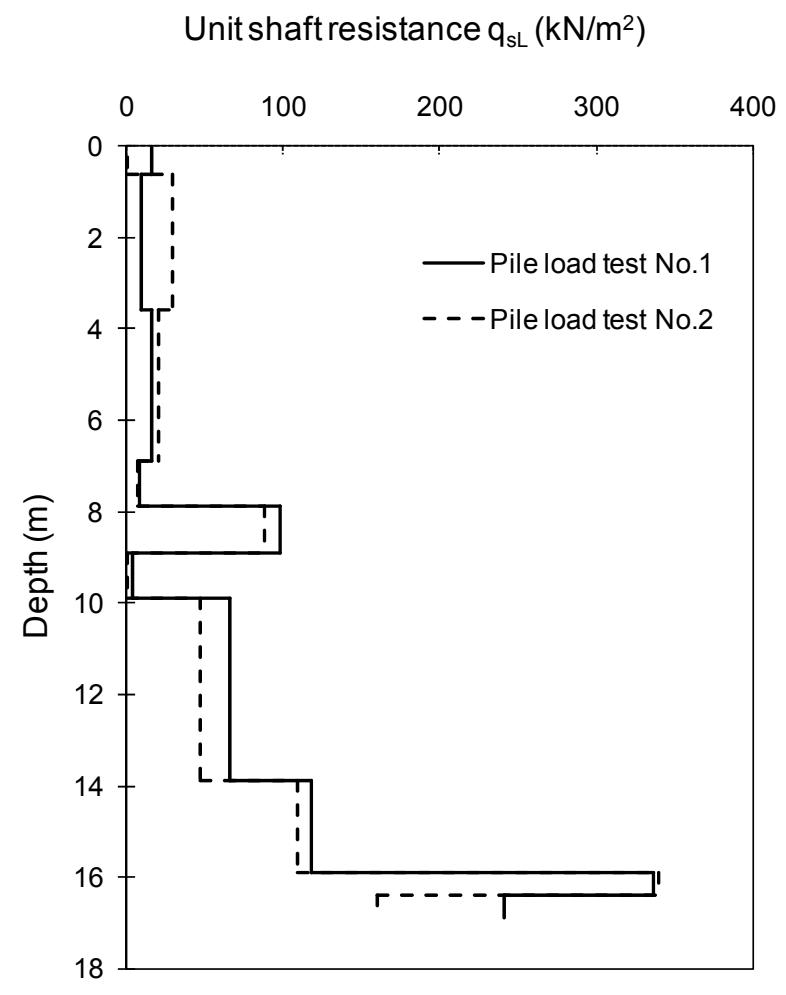

Figure 6.14. Unit shaft resistance versus depth. 


\subsection{Model pile load tests}

\subsubsection{Experimental setup}

The model pile load tests were conducted at the Bowen Laboratory for Large-Scale Civil Engineering Research at Purdue University. The purpose of the model tests was to obtain dynamic and static pile load data from tests performed under fully controlled conditions, avoiding the large uncertainties that are inherent in natural soil profiles. Two model piles and a drop hammer driving system were manufactured specifically for these tests. Both model piles were close-ended pipe piles. The first pile (model pile I) is made of mild steel and is $1.05 \mathrm{~m}$ long. Its outer diameter is $33.4 \mathrm{~mm}$ with wall thickness $4.54 \mathrm{~mm}$. The average roughness $R_{\mathrm{a}}$ of mild steel is in the $5 \mu \mathrm{m}$ to $10 \mu \mathrm{m}$ range. The second pile (model pile II) is made of stainless steel and is $1.10 \mathrm{~m}$ long. The outer diameter and the wall thickness of the model pile II is $30.2 \mathrm{~mm}$ and $2.1 \mathrm{~mm}$, respectively. The roughness $R_{\mathrm{a}}$ of pile II is $0.63 \mu \mathrm{m}$.

The model piles were driven by a $3.06 \mathrm{~kg}$ hammer with drop height ranging from $0.5 \mathrm{~m}$ to $1.0 \mathrm{~m}$. The model pile and drop hammer assembly schematics are shown in Fig. 6.15. The closing end at the base of the model piles was configured so that it can function as a load cell (Figure A.7 in the Appendix). The model piles were instrumented with two piezoelectric accelerometers attached to the pile head. Pile I was also instrumented with six electric resistance strain gages. Two of the strain gages and the two accelerometers were installed diametrically opposite approximately two diameters below the pile head. The other four strain gages were placed in the specially configured base. Pile II was instrumented with nine pairs of strain gages at the shaft and four strain gages at the base (Fig. 6.16). However, the strain gages that were attached to the pile shaft gave unreliable results (negative shaft resistance, large difference between gages in the same pair). Therefore, the determination of load transfer curves along the shaft was not possible in the model pile tests. A list of the model pile load tests is given in Table 6.2.

\subsubsection{Soil properties}

The first two tests in Table 6.2 were done with medium grained Ottawa sand $\left(D_{50}=0.39 \mathrm{~mm}\right)$ designated as ASTM C778. This type of Ottawa sand has been tested extensively at Purdue University and its small- and large- strain soil properties are well-known. It is a uniform quartz sand (coefficient of uniformity $C_{\mathrm{u}}=1.43$ ) with round to subround particles. The 
maximum and minimum void ratios $e_{\max }$ and $e_{\min }$ are 0.78 and 0.48 , respectively (Carraro et al., 2003). The critical-state friction angle $\phi_{c}$ in triaxial compression is $30.2^{\circ}$ (Murthy et al., 2007). Based on bender element test data, the small-strain shear modulus of Ottawa sand is given by the following equation (Carraro et al., 2003; Carraro, 2004):

$$
G_{\max }=611 \frac{(2.17-e)^{2}}{1+e} p^{\prime 0.437} p_{\mathrm{A}}^{0.563}
$$

where $e$ is the void ratio, $p^{\prime}$ is the mean effective stress, and $p_{\mathrm{A}}$ is a reference stress $(=100 \mathrm{kPa})$.

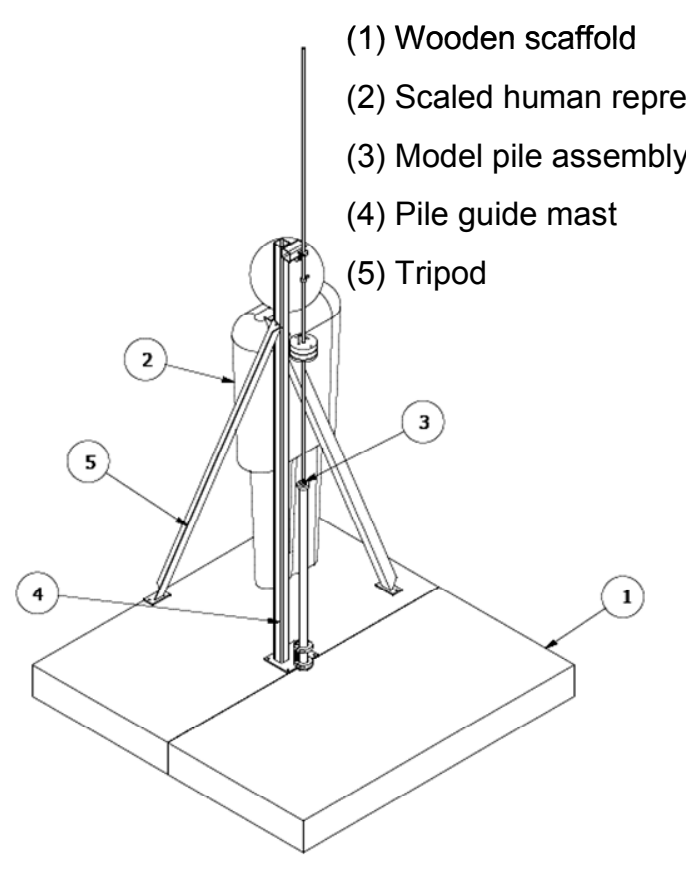

(a) Pile load cell

(b) Model pile

(c) Pile cap

(d) Drop masses

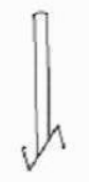

(e) Drop hammer guide
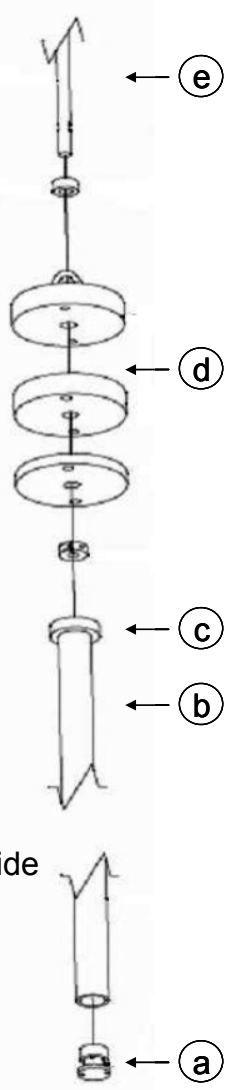

Figure 6.15. Model pile and drop hammer assembly 


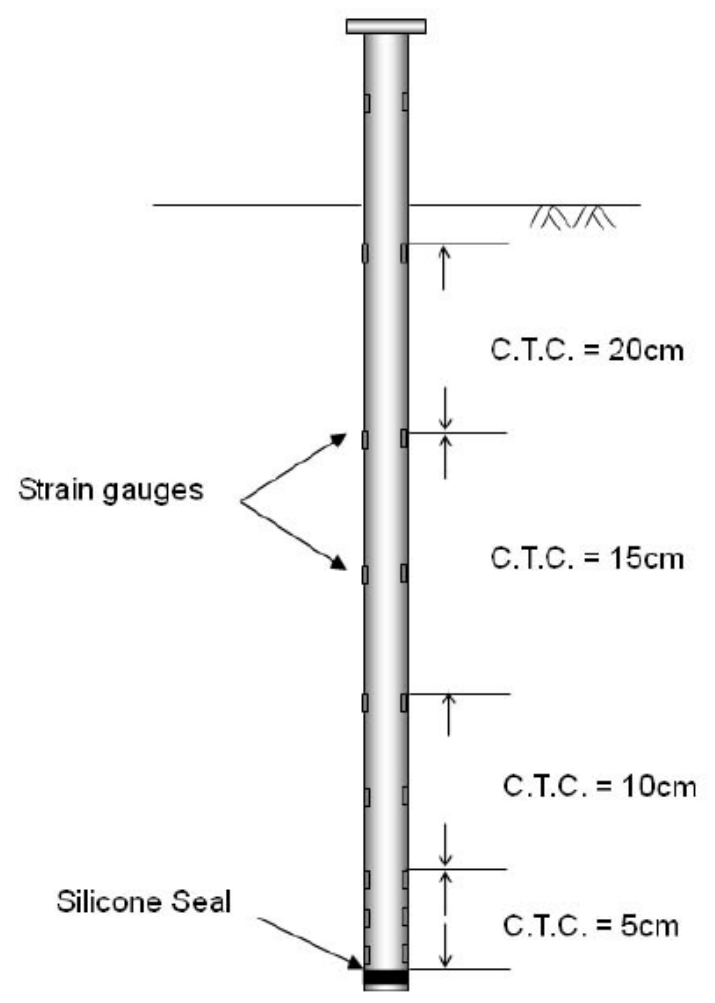

Figure 6.16. Arrangement of strain gages in model pile II

Table 6.2. List of model pile tests (tests 3-6 after Lee 2008)

\begin{tabular}{|c|c|c|c|c|c|}
\hline Test \# & Location & Pile & Sand & $\begin{array}{c}\text { Relative } \\
\text { density }\end{array}$ & $\begin{array}{c}\text { Pile } \\
\text { penetration } \\
\text { depth }(\mathrm{m})\end{array}$ \\
\hline 1 & test pit & $\mathrm{I}$ & medium & $\mathrm{D}_{\mathrm{R}}=20 \%$ & 0.86 \\
\hline 2 & test pit & $\mathrm{I}$ & medium & $\mathrm{D}_{\mathrm{R}}=95 \%$ & 0.70 \\
\hline 3 & test pit & $\mathrm{II}$ & fine & $\mathrm{D}_{\mathrm{R}}=61 \%$ & 0.96 \\
\hline 4 & test pit & $\mathrm{I}$ & fine & $\mathrm{D}_{\mathrm{R}}=36 \%$ & 0.94 \\
\hline 5 & test pit & $\mathrm{II}$ & fine & $\mathrm{D}_{\mathrm{R}}=80 \%$ & 0.89 \\
\hline 6 & soil tank & $\mathrm{II}$ & fine & $\mathrm{D}_{\mathrm{R}}=91 \%$ & 0.92 \\
\hline
\end{tabular}


In later tests, fine-grained Ottawa sand $\left(D_{50}=0.23 \mathrm{~mm}\right)$ was used. This sand is also uniform and rounded, with maximum and minimum void ratios $e_{\max }$ and $e_{\min }$ of 0.78 and 0.47 , respectively. Drained triaxial compression tests showed that the critical-state friction angle for the fine sand was roughly $32^{\circ}$. Data from small-strain tests is not available yet. However, since the fine Ottawa sand has strong similarities (particle angularity, limiting void ratios) to the medium one, it is reasonable to assume that the $G_{\max }$ value will not be much different from that predicted by eq. (6.1).

Most of the pile load tests was performed outside the Bowen Laboratory building, where a cylindrical test pit was excavated. Both diameter and depth of the pit were roughly $1.3 \mathrm{~m}$. One test (No.6) was performed in the soil tank inside the Bowen Laboratory building. The cylindrical soil tank has diameter $2.0 \mathrm{~m}$ and height $1.6 \mathrm{~m}$. The dimensions of test pit and soil tank were chosen based on the studies of the calibration chamber size needed for boundary effect on pile bearing capacity and cone penetration resistance to be small. Parkin and Lunne (1982) and Salgado et al. (1998) suggested that the diameter of the calibration chamber must be at least 50 times and 100 times the cone diameter, respectively. Even though the literature suggests a test pit dimension at least 50 times the pile diameter (about $1.6 \mathrm{~m}$ ), the pit diameter of $1.3 \mathrm{~m}$ is still acceptable because the pit wall was left unlined. This can be seen as the native soil taking the place of Ottawa sand beyond $0.65 \mathrm{~m}$ from the pit center. Hence, the actual boundary condition is less severe than a fully rigid or fully flexible (inflated membrane) calibration chamber wall.

The model pile tests were performed in dry soil samples for various relative density values. In test No.1, the very loose sample $\left(D_{R}=20 \%\right)$ was achieved by emptying the sand bags into the test pit. The very dense sample $\left(D_{R}=95 \%\right)$ of test No.2 was prepared by using a vibrating plate compactor. The sand was placed in lifts and the compaction effort was controlled in order to achieve a homogeneously dense sample throughout the pit. During the preparation of the very dense sample, an earth pressure cell was placed in the soil at a depth of $40 \mathrm{~cm}$ and close to the pit wall, in order to measure the actual at-rest earth pressure coefficient $\left(K_{0}\right)$. This was done because, due to compaction, the sand will have experienced larger stress than the in situ stress during pile testing (OC sand). The $K_{0}$ of an NC sand can be estimated adequately since the possible value falls in a relatively narrow range ( 0.4 to $0.5)$. The $K_{0}$ cannot be guessed in the case for OC sand. Hence, a disk-type lateral earth pressure cell was placed at $0.5 \mathrm{~m}$ depth and close to the wall of the pit before placement of the sand. $A$ value of $K_{0}=1.1$ was measured after the pit was filled to the top with compacted sand. The $K_{0}$ value is necessary for calculating mean effective stress, which is in turn used 
for calculating the small-strain shear modulus and the limit base resistance used in the 1-D pile driving simulations. In the other pile load tests, the samples were prepared by air pluviation using a pluviator that was manufactured specifically for soil tank tests. The use of the pluviator helped in producing sand profiles that are much more uniform than those in the first two tests. In all cases, the density of the sand is estimated from the weight of the sand placed in the pit and the volume of the pile.

\subsubsection{Pile test results}

Typical pile driving records (pile penetration per blow versus depth of penetration) are shown in Figs. 6.17 and 6.18. The acceleration at the pile head was captured using the Pile Driving Analyzer (PDA). An oscilloscope was used to record the signals from the strain gages. The acceleration histories were recorded for every blow as the model pile was driven into the ground. Typical displacement and velocity time histories are shown in Fig. $6.19,6.20,6.21$, and 6.22. Displacement and velocity time histories are calculated automatically by PDA from the measured acceleration time history using numerical integration.
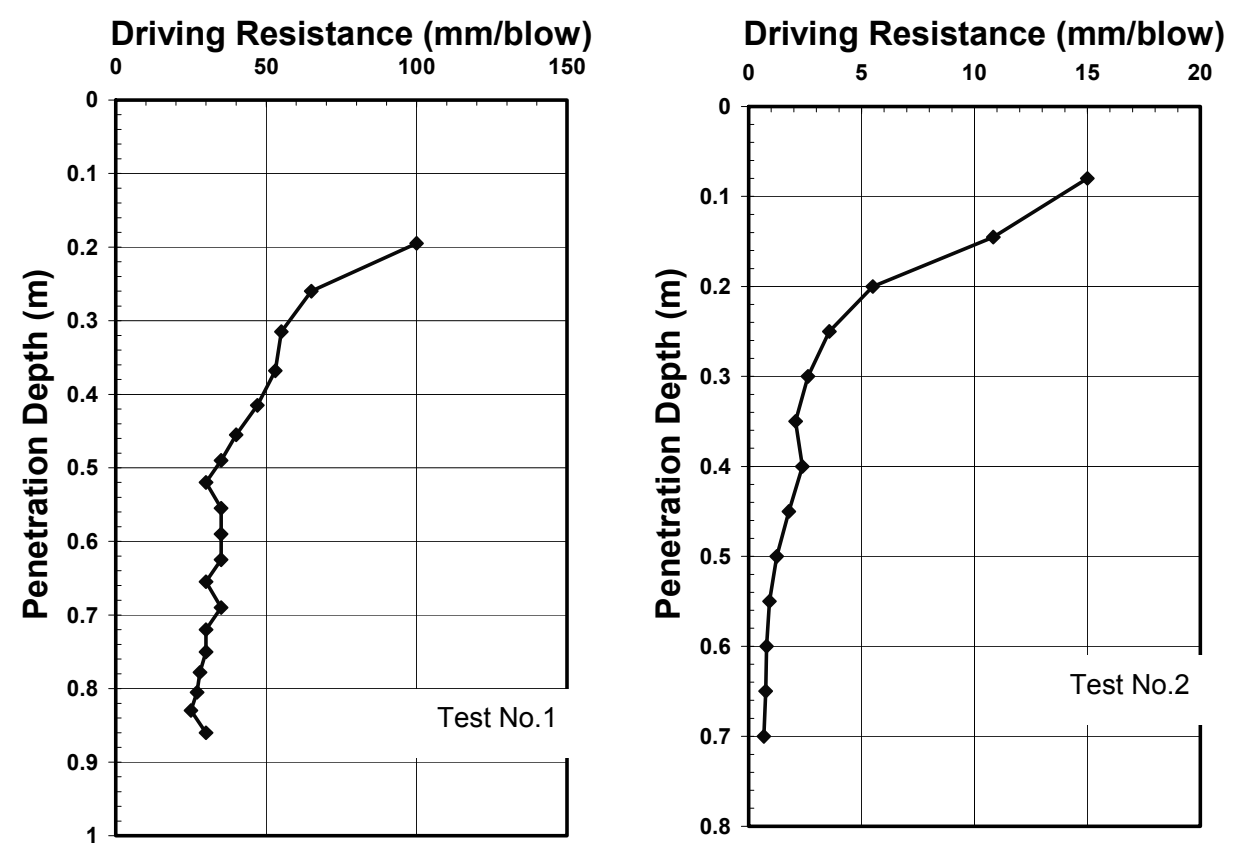

Figure 6.17. Driving resistance in Tests No.1 and No.2 

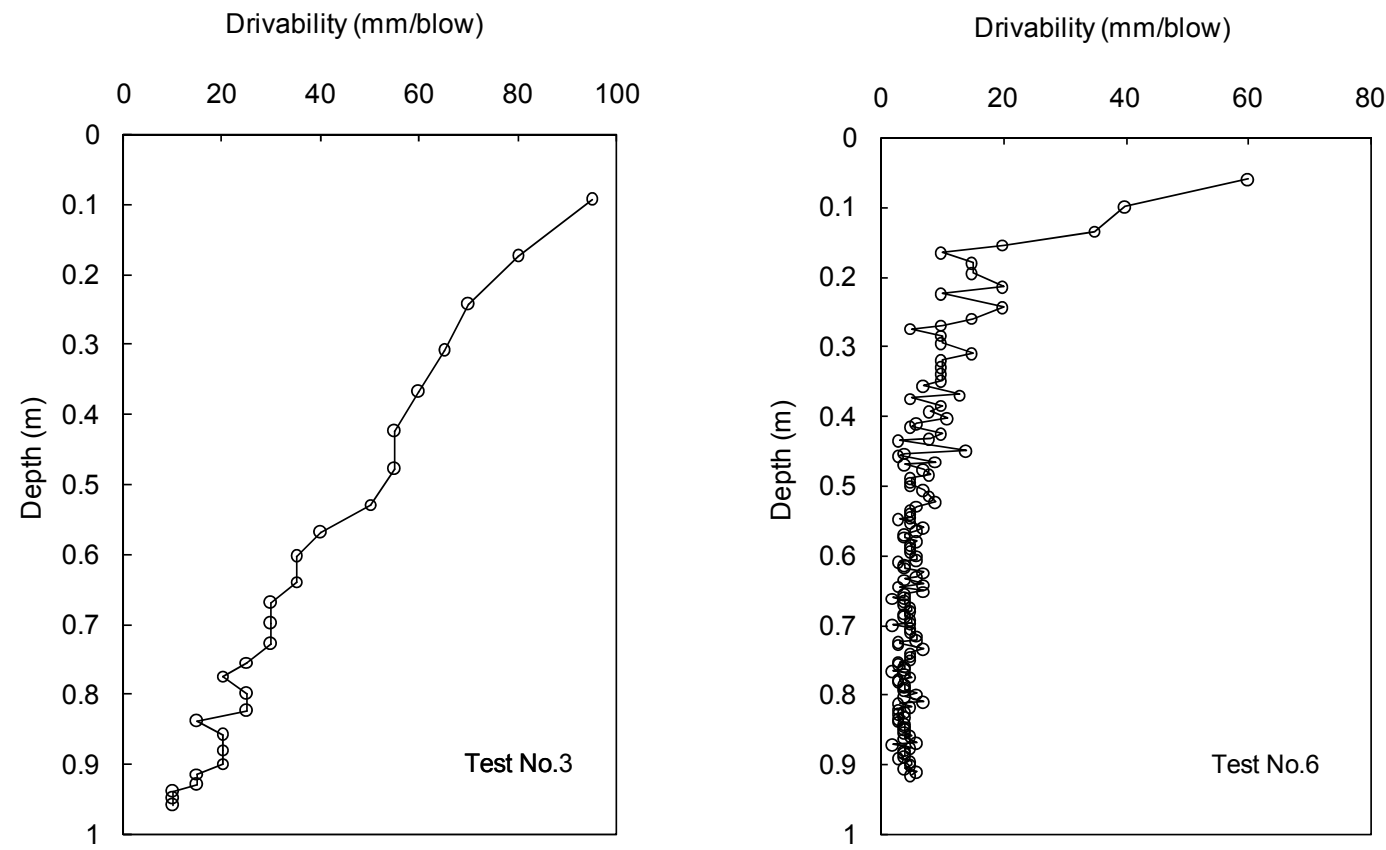

Figure 6.18. Driving resistance in Tests No.1 and No.2
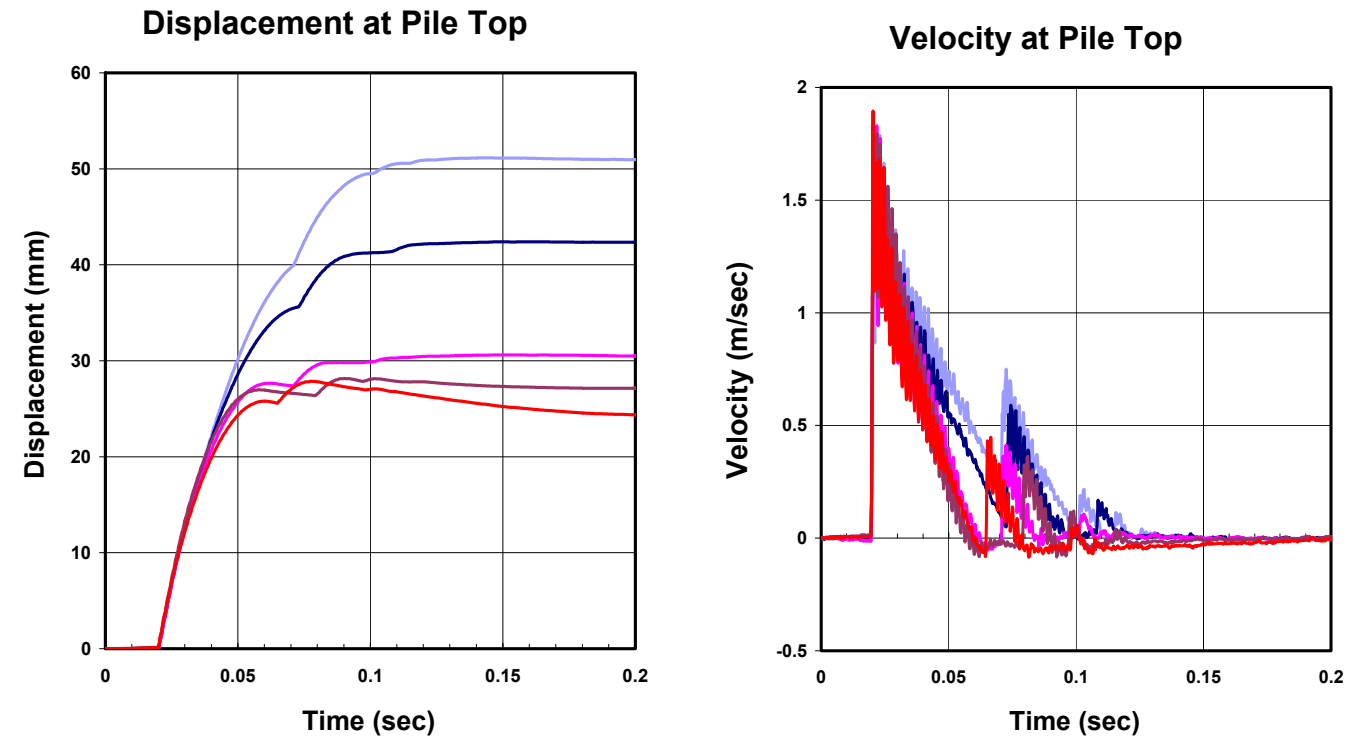

$$
\begin{aligned}
& - \text { Blow \#3 }(\sim 30 \mathrm{~cm}) \\
& - \text { Blow \#5 }(\sim 40 \mathrm{~cm}) \\
& - \text { Blow \#10 }(\sim 60 \mathrm{~cm}) \\
& - \text { Blow \#13 }(\sim 70 \mathrm{~cm}) \\
& \text { Blow \#17( } \sim 80 \mathrm{~cm})
\end{aligned}
$$

Figure 6.19. Displacement and velocity records from Test No.1 
Displacement at Pile Top

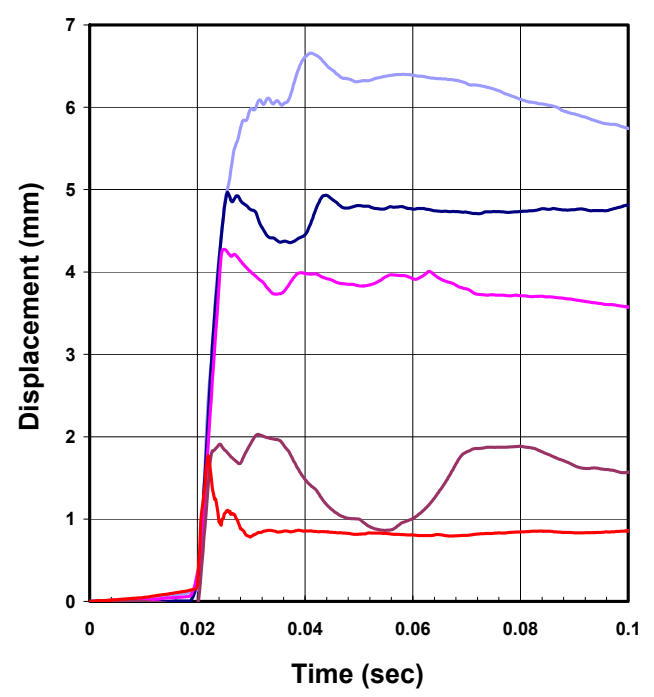

Velocity at Pile Top

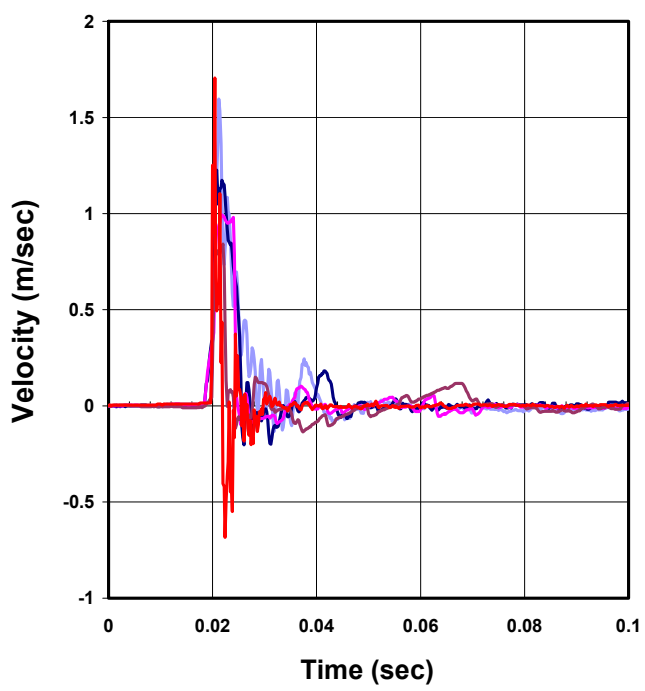

Blow \#10 ( 15cm)

Blow \#17 ( 19.5cm)

Blow \#25 ( 25cm)

Blow \#74 ( 35cm)

Blow \#392 ( 70cm)

Figure 6.20. Displacement and velocity records from Test No.2

The procedure followed during the static load tests on the model piles was similar to the one used in field test. In the case of the tests performed in the sand pit, the reaction was provided by dead weights. Two concrete blocks were placed on the two sides of an $\mathrm{H}$-beam that serves as the reaction beam (Fig, A.12). In the case of the test in the soil tank, the reaction beam was firmly attached to the walls of the tank, which provided the necessary reaction. The hydraulic jack and a load cell were placed between the pile and the reaction beam (Fig. A.11). The total load applied to the pile head was measured by a load cell with a capacity of $20 \mathrm{kN}$.

The CR5000 Campbell Scientific datalogger was used to read the strain gage signals. Dial gages were attached to two reference beams placed on the two sides of the pile in order to monitor the pile settlement. The measured shaft and base capacities are presented in Table 6.3. Typical axial load-settlement curves are shown in Fig. 6.23 and 6.24 . 

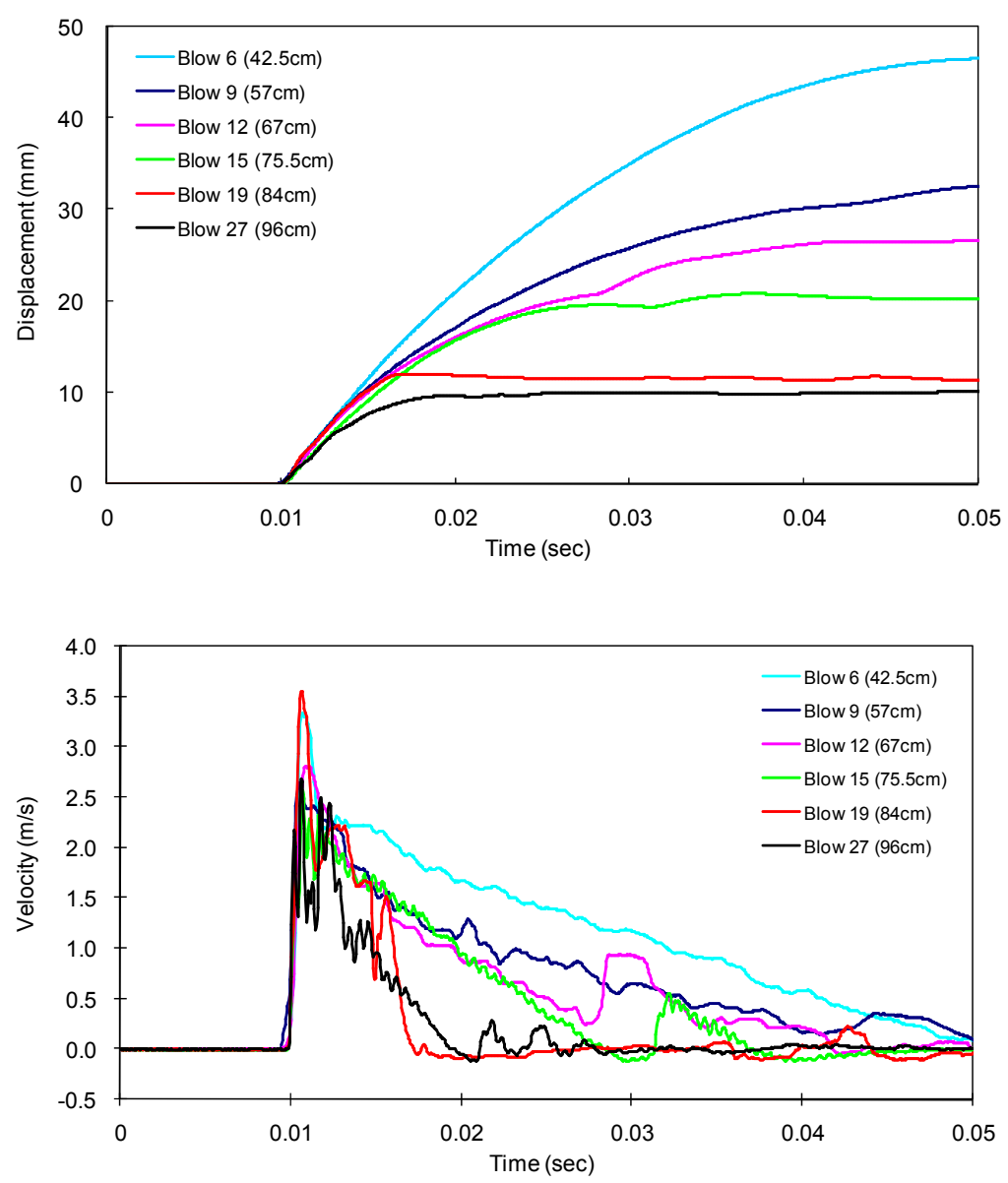

Figure 6.21. Displacement and velocity records from Test No.3

Table 6.3 Shaft and base capacities from the static load tests on model piles

\begin{tabular}{|c|c|c|}
\hline Test \# & $\mathrm{Q}_{\mathrm{sL}}(\mathrm{kN})$ & $\mathrm{Q}_{\mathrm{b}, \text { ult }}(\mathrm{kN})$ \\
\hline 1 & 0.30 & 0.07 \\
\hline 2 & 4.72 & 5.50 \\
\hline 3 & 0.78 & 0.73 \\
\hline 4 & 0.14 & 0.17 \\
\hline 5 & 0.97 & 1.09 \\
\hline 6 & 1.65 & 2.72 \\
\hline
\end{tabular}



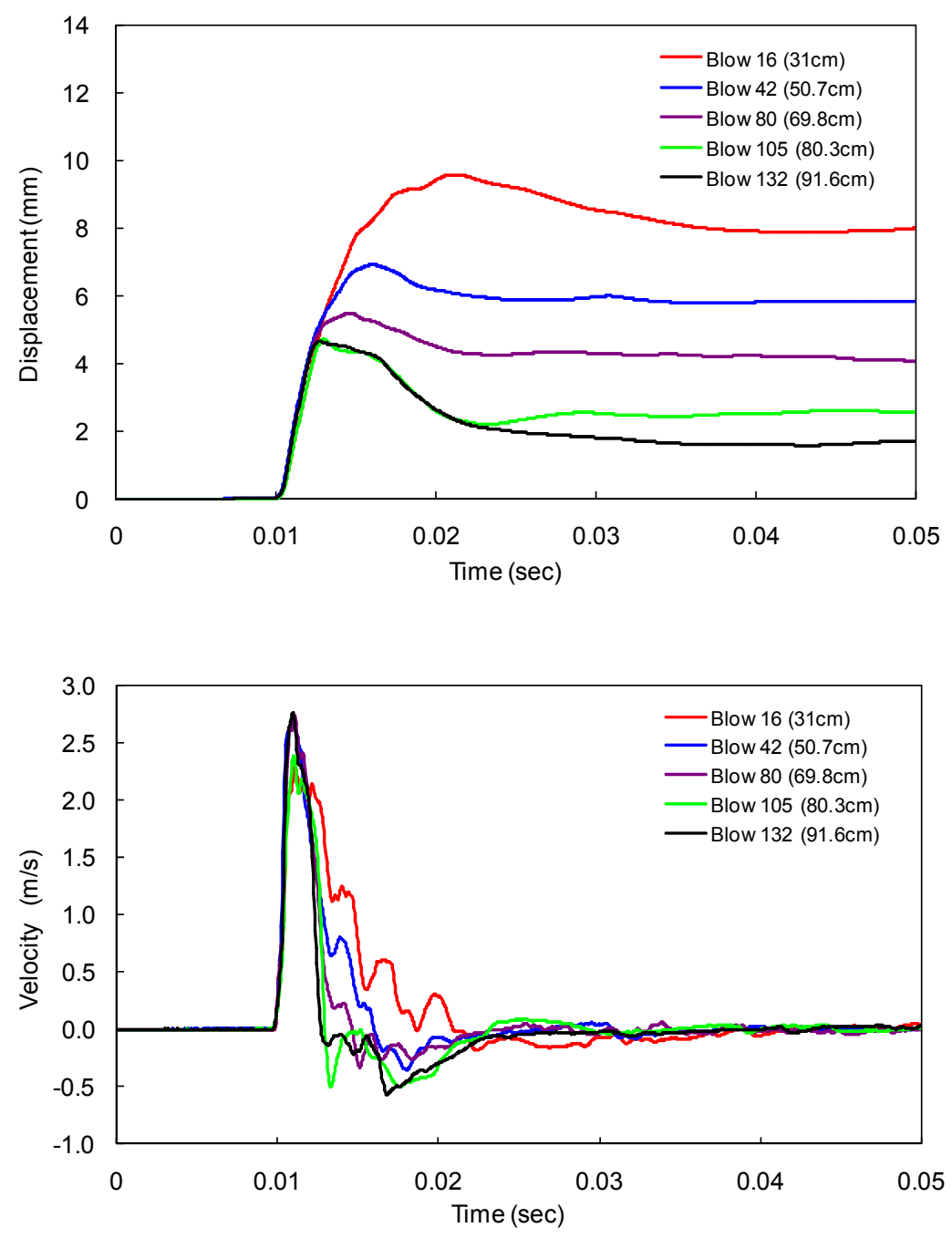

Figure 6.22. Displacement and velocity records from Test No.6 

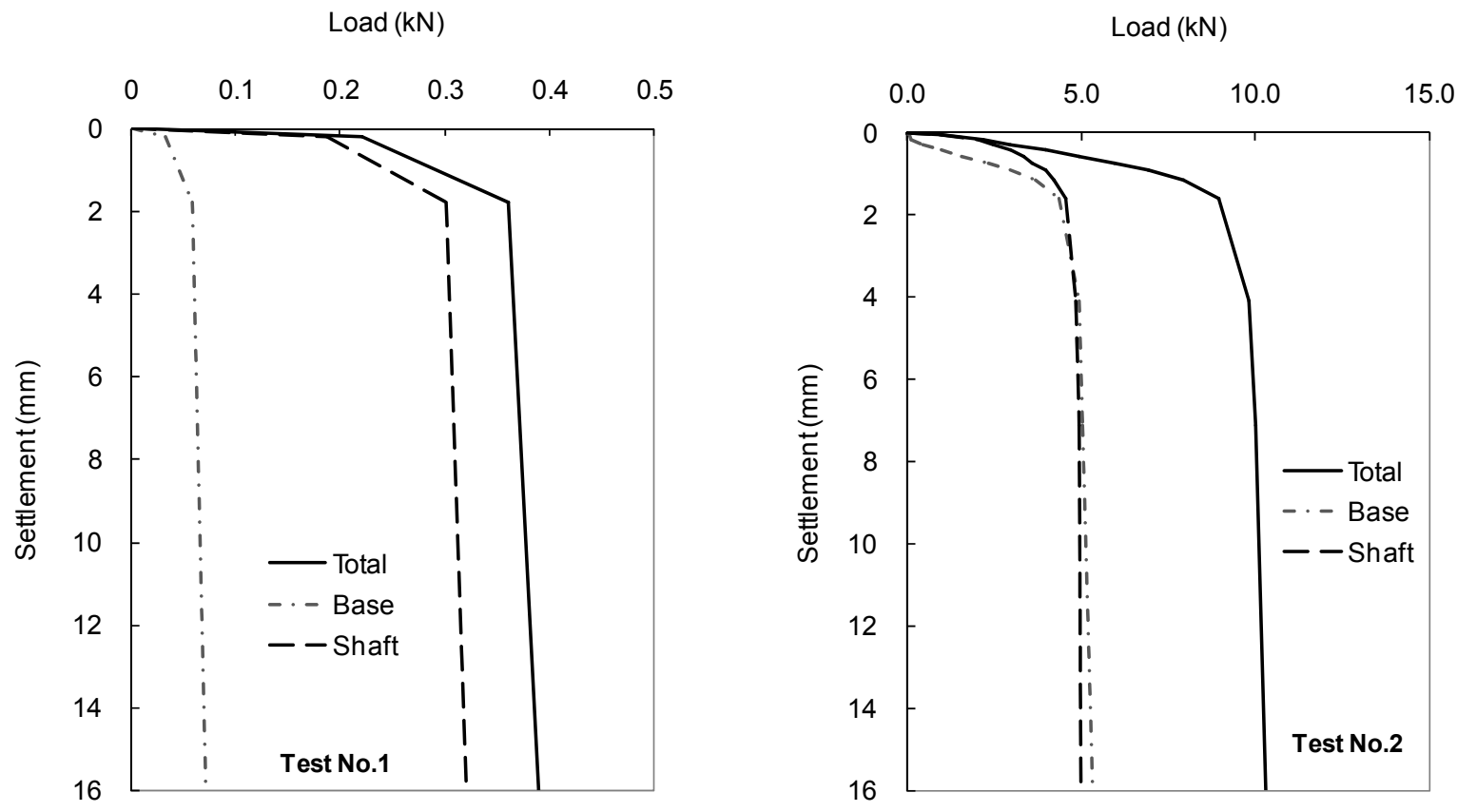

Figure 6.23. Load-settlement curves for static load tests No. 1 and No. 2
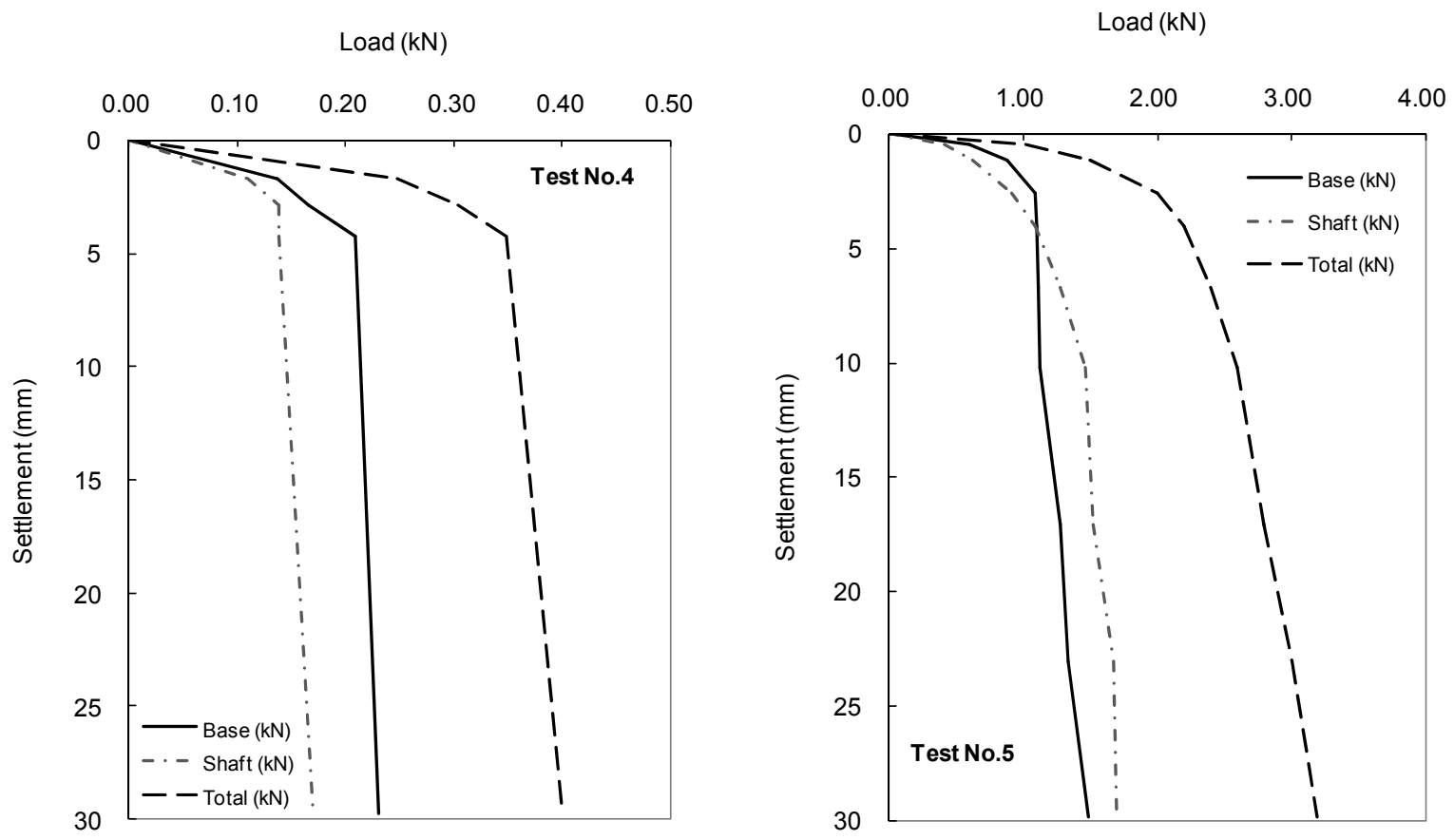

Figure 6.24. Load-settlement curves for static load tests No. 4 and No. 5 


\subsection{Field pile load test in Lagrange County}

Full-scale pile load tests were conducted at the bridge construction project over the Pigeon River, on State Road 9, in Lagrange County. Details of this experimental program can be found in Paik et al. (2003). The testing program included pile driving monitoring, restrike tests, and static load tests. The tests were performed on two instrumented pile, one closedended steel pipe pile and one open-ended steel pipe pile. Herein, we will focus on the closed-ended pipe pile. The pile was instrumented with two accelerometers and two strain transducers at the pile head to obtain the acceleration and force time histories during driving. Eighteen strain gages grouped in pairs were attached at nine levels along the pile shaft in order to measure the strain in the pile during static loading. The site exploration, which included SPT and CPT testing (Figure 6.25), showed that the natural soil profile consisted of loose gravelly sand $\left(D_{\mathrm{R}}=30 \%\right)$ down to $3 \mathrm{~m}$ depth, followed immediately by dense gravelly sand with $D_{\mathrm{R}}=80 \%$. The water table was at $3 \mathrm{~m}$ depth. The pile was $8.24 \mathrm{~m}$ long and had outer and inner diameters of $356 \mathrm{~mm}$ and $330.6 \mathrm{~mm}$, respectively.

The pile was driven down to $6.9 \mathrm{~m}$ depth using an ICE-42S single acting diesel hammer. The ram weight was $18.2 \mathrm{kN}$ and the maximum hammer stroke was $3.12 \mathrm{~m}$. The rated maximum driving energy was $56.8 \mathrm{~kJ}$. Data recorded during driving using the PDA showed that the energy transferred to the pile head ranged from $19.0 \mathrm{~kJ}$ to $28.5 \mathrm{~kJ}$, i.e. $33 \%$ to $50 \%$ of the rated (potential) hammer energy. The pile set per blow ranged from $300 \mathrm{~mm}$ during early stages of driving to $10 \mathrm{~mm}$ near the end of the installation. The reaction system used in the static pile load tests consisted of six reaction piles. According to the static load test results, the limit base resistance of the closed-ended pipe pile is $12166 \mathrm{kPa}$. The limit unit shaft resistance calculated based on the strain gage data ranges from $17 \mathrm{kPa}$ to $132 \mathrm{kPa}$ (Figure 6.26). 


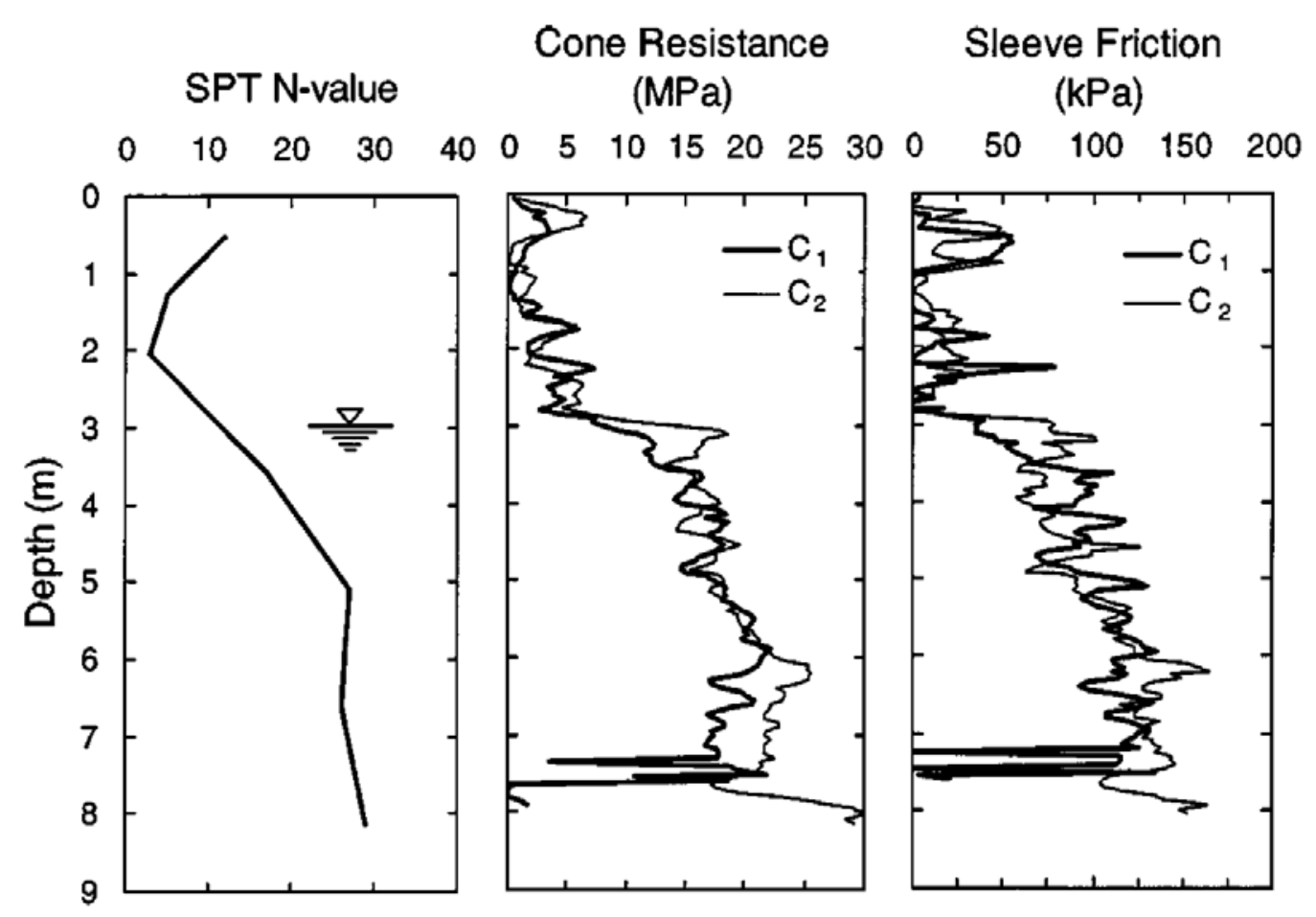

Figure 6.25. SPT and CPT data for test site at Lagrange County

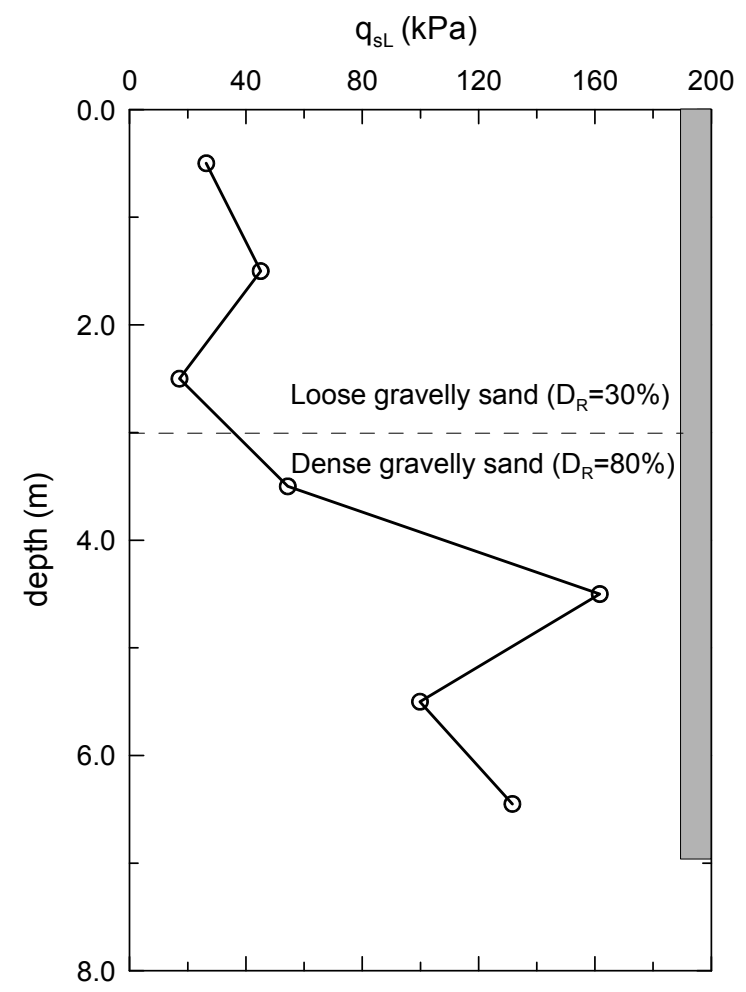

Figure 6.26. Distribution of limit unit shaft resistance along pipe pile at Lagrange County. 


\section{CHAPTER 7. NUMERICAL PREDICTIONS USING PROPOSED MODELS AND COMPARISON WITH PILE DRIVING DATA}

In this Chapter, we simulate the field and model pile load tests presented in Chapter 6. The pile driving simulations use the new shaft and base reaction models presented in Chapter 5 . The comparison between the numerical predictions and the observed pile driving data is done in order to validate the proposed soil reaction models. The predictions using the new soil reaction models are also compared with those using the Smith (1960) shaft and base reaction models, which constitute the core of dynamic pile analyses as currently done in design practice. The simulations compute the pile set given the hammer energy transmitted to the pile head. The hammer energy inputted in the simulations accounts for the energy losses inside the hammer system before impact and the energy losses during the impact as thermal energy and scattered transverse waves in the anvil, pile helmet and cushions. All analyses are done for the last few hammer blows (i.e., pile installation is complete or nearly complete), and so there are pile load test data on the static resistance of the pile (which is an inseparable part of its dynamic resistance and thus of the driving analyses). Validating the numerical model for the case of a pile at its final penetration depth is of most importance because back-calculation of the pile capacity in practice through signal matching is done for a fully installed pile.

\subsection{Field pile load test in Jasper County}

According to GRLWEAP, the efficiency of open-ended (single acting) diesel hammers is 0.8 . This efficiency factor accounts only for hammer energy losses before impact. The PDA measurements showed that the energy transmitted from the open-end diesel hammer to the pile head at the last stages of driving was only $36 \%$ of the maximum potential (rated) energy of the hammer. Since we are not modeling explicitly the parts that lie between the ram and the pile head, and, consequently, the energy losses inside and at the interfaces of these parts, we scale the rated hammer energy by an efficiency factor $e_{f}$ (eq. 5.64) equal to 0.36 . The pile is divided into 19 segments. The input unit shaft and base resistances are based on the results of the first static load test, which was performed 50 days after installation. These 
unit resistance values may include some amount of pile setup, which would lead to a slight underprediction of the pile displacement from the dynamic analyses. The maximum soil shear modulus $G_{\max }$ in the case of silty clay and clayey silt was estimated based on the undrained shear strength using the correlation by Weiler (1988) (eq. 5.45). In the case of silty sand, clayey sand, and stiff silt, $G_{\max }$ was calculated using the CPT- based correlation by Rix and Stokoe (1991) (Table 5.5). The coefficient of lateral earth pressure at rest $K_{0}$ in the sand or nonplastic silt layers was assumed equal to 0.45. The small-strain Poisson's ratio of the soil at the base was assumed to be equal to 0.5 . The coefficient $b_{\mathrm{fb}}$ in eqs. (5.27) and (5.39) was set equal to 1.5 based on the load-settlement curves of the static load test. The soil viscosity parameters were set to $m_{\mathrm{b}}=m_{\mathrm{s}}=0.3$ and $n_{\mathrm{b}}=n_{\mathrm{s}}=0.2$ for the clayey and silty sand and the stiff silt. Eqs. (5.47) and (5.48) were used for calculating the soil viscosity parameters in the case of cohesive soils (silty clay and clayey silt).

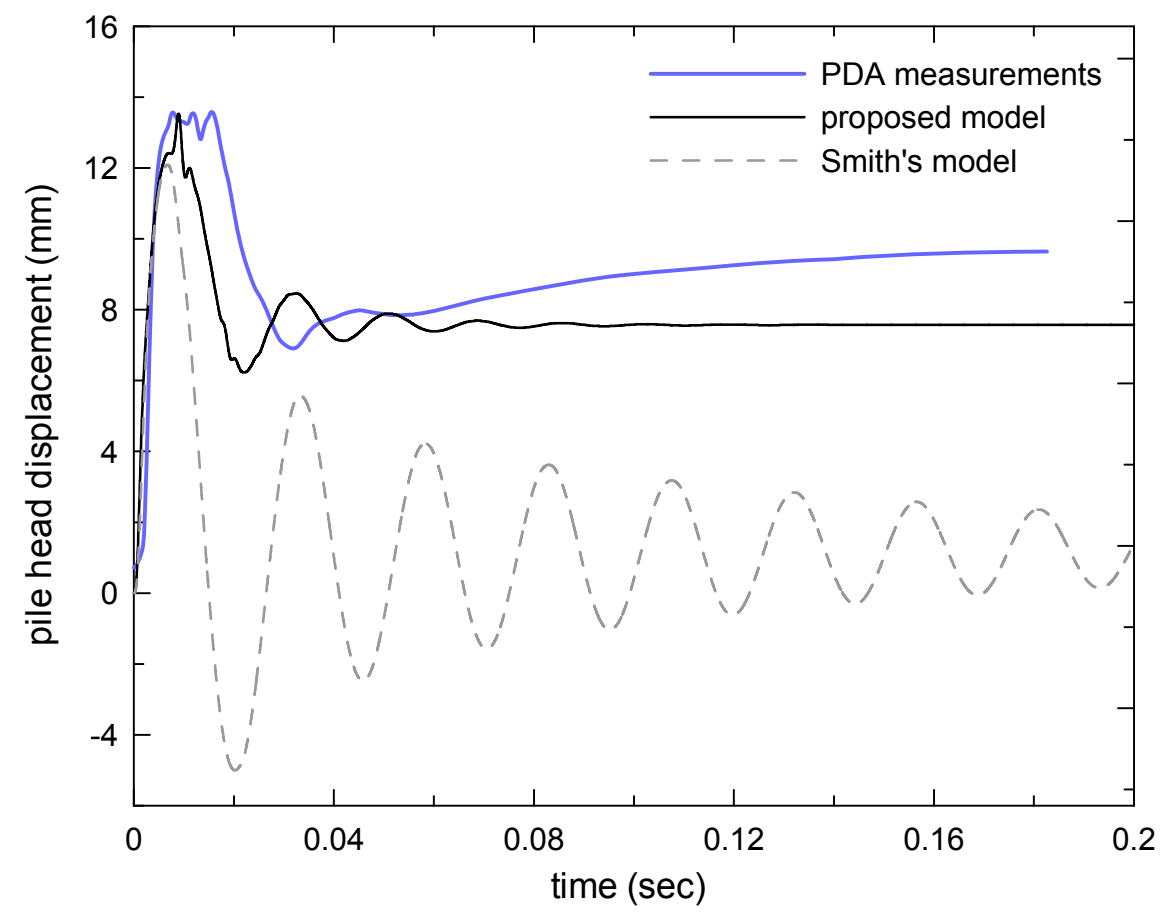

Figure 7.1. Predicted and measured pile head displacement time history for closed-ended steel pipe pile in Jasper County.

Figure 7.1 compares the predicted displacement time history at the pile head from the simulations using the proposed soil reaction models with that recorded during pile 
driving using the PDA. The prediction using Smith (1960) reaction models are also plotted in the figures. The analyses using the proposed model predict final set equal to $7.5 \mathrm{~mm}$, while, according to PDA measurements, the final set is $9 \mathrm{~mm}$. The analyses using Smith's model largely underpredict the final set and yield pile head motion characterized by large amplitude oscillations. In contrast, the shape of the displacement time history curve from the proposed model is similar to the observed one.

From the many variables describing the pile motion, the pile set, which is the residual displacement after all oscillations have dissipated, is of most importance. This is because the pile set depends directly on the overall energy losses inside the soil, and, consequently, on the work done by the shaft and base resistance. Adequate representation of the work done by the soil resistance in dynamic pile analysis is required for the effective backcalculation of the pile capacity using signal matching.

\subsection{Model pile load tests}

The model piles were driven using a drop hammer. According to Bowles (1996), the efficiency of drop hammers ranges from 0.75 to 1.0 , while the software GRLWEAP suggests a value of 0.67 . The hammer in the model tests was donut-shaped and was allowed to fall free without a rope or string attached to it. According to the experimental study by Morgano and Liang (1992) on safety hammers attached to rope and pulley, $20 \%$ of the initial potential hammer energy is lost before impact (i.e. the kinetic energy of the hammer right before impact is 0.8 times the initial potential energy) and another $16 \%$ is lost during the impact (for short rod length and resistance up to $4.5 \mathrm{kN}$, similar conditions to the model pile tests), resulting in a hammer efficiency factor of 0.67 . Measurements on free falling (no rope and pulley) SPT donut hammers in Japan yielded a hammer efficiency factor equal to 0.78 . (Bowles, 1996). Based on the above, we will use a hammer efficiency factor $e_{f}$ equal to 0.75 . The piles were divided into 8 to 11 segments.

The total shaft capacity measured in the static load tests was distributed along the pile shaft by first calculating the limit unit shaft resistance values using the method by Foye et al. (2006) for displacement piles (Chapter 3 ) based on the sand relative density and the effective confining stress. Then, the computed limit unit shaft resistance distribution was scaled so that the corresponding shaft capacity matched the one observed in the pile load tests. The pile used in the first two model tests (pile I) was made of mild steel. Hence, the 
coefficient $n_{\mathrm{F}}$ in eq. (5.23) was set equal to 1.22 for pile I. This value reflects the fact that the ratio of the mild steel-sand interface friction angle to the critical-state friction angle of the soil is 0.85 . The model pile used in the remaining tests (pile II) was made of stainless steel with a much smoother shaft wall surface. Based on the experimental study of Ling and Dietz (2005) on the strength of sand-steel interfaces, the interface friction angle is estimated to be 0.5 times the critical state friction angle of the sand for the measured average roughness of $0.63 \mu \mathrm{m}$ and the $D_{50}$ of the fine Ottawa sand. Hence, the coefficient $n_{\mathrm{F}}$ for pile II was set equal to 2.0. The maximum shear modulus of the sand was estimated using eq. (6.1). The small-strain Poisson's ratio was assumed to be equal to 0.15 . It was found that the static base resistance vs. base settlement curves are adequately fitted by using a coefficient $b_{\mathrm{fb}}$ equal to 2.0. The soil viscosity parameters were set to $m_{\mathrm{b}}=m_{\mathrm{s}}=0.3$ and $n_{\mathrm{b}}=n_{\mathrm{s}}=0.2$.

Figures 7.2 through 7.7 compare the predicted displacement time history at the pile head from the simulations using the proposed soil reaction models with that recorded during pile driving using the PDA. The prediction using Smith (1960) reaction models are also plotted in the figures. The values for quake and damping coefficients in the simulations using Smith's models were set equal to those recommended in the GRLWEAP manual $\left(Q_{\mathrm{s}}=2.5 \mathrm{~mm}, Q_{b}=B / 120, J_{\mathrm{s}}=0.16 \mathrm{~s} / \mathrm{m}\right.$, and $\left.J_{b}=0.5 \mathrm{~s} / \mathrm{m}\right)$.

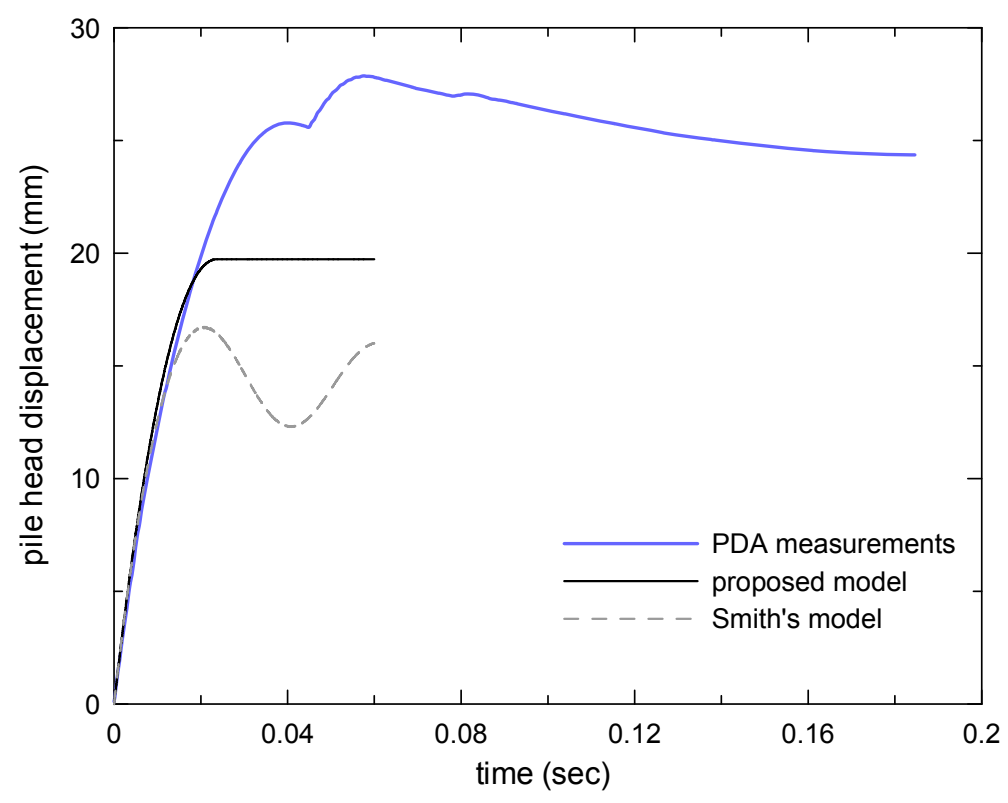

Figure 7.2. Predicted and measured pile head displacement time history for model pile test No.1: medium Ottawa sand with $D_{\mathrm{R}}=20 \%$. 


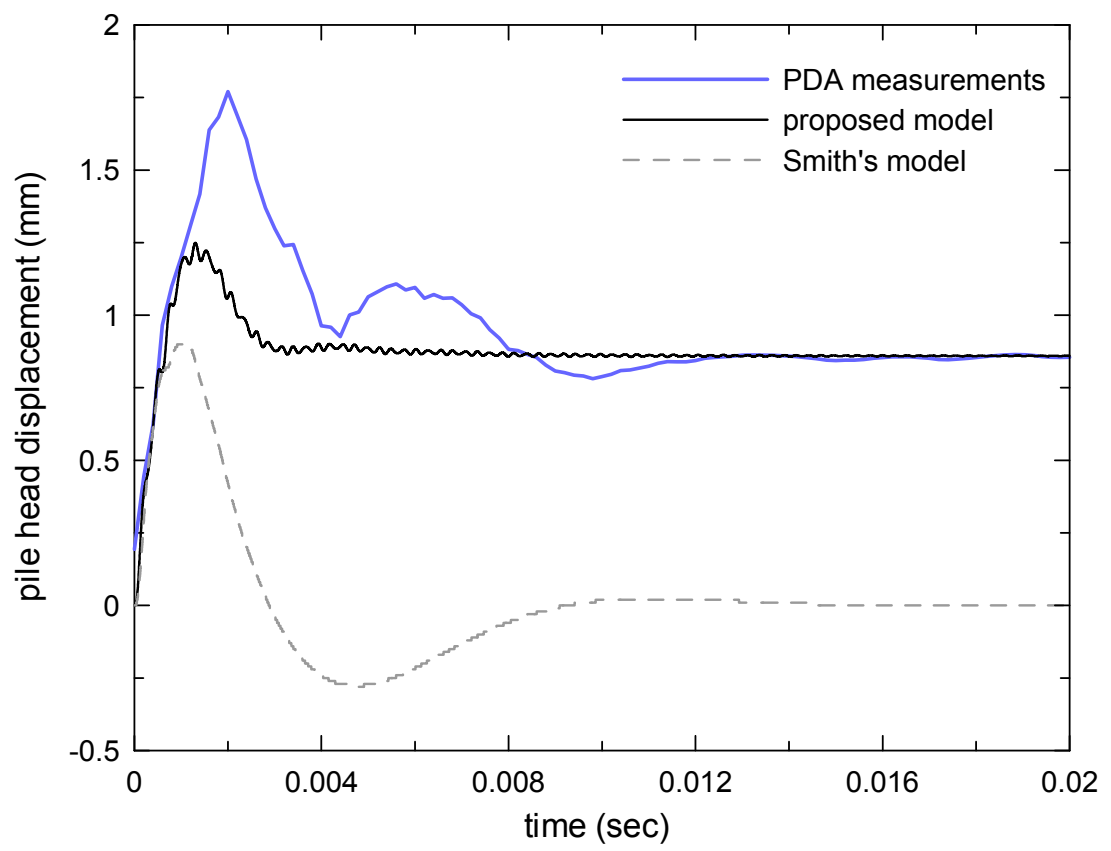

Figure 7.3. Predicted and measured pile head displacement time history for model pile test No.2: medium Ottawa sand with $D_{\mathrm{R}}=95 \%$.

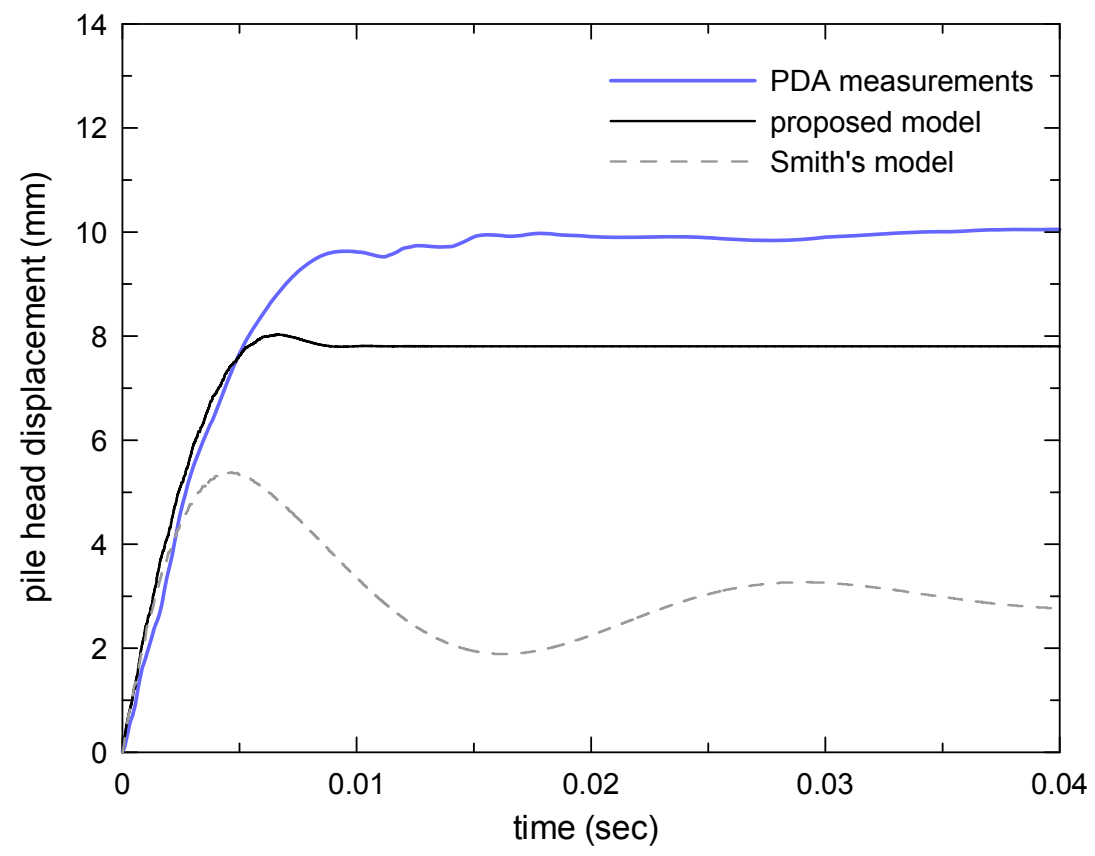

Figure 7.4. Predicted and measured pile head displacement time history for model pile test No.3: fine Ottawa sand with $D_{\mathrm{R}}=61 \%$. 


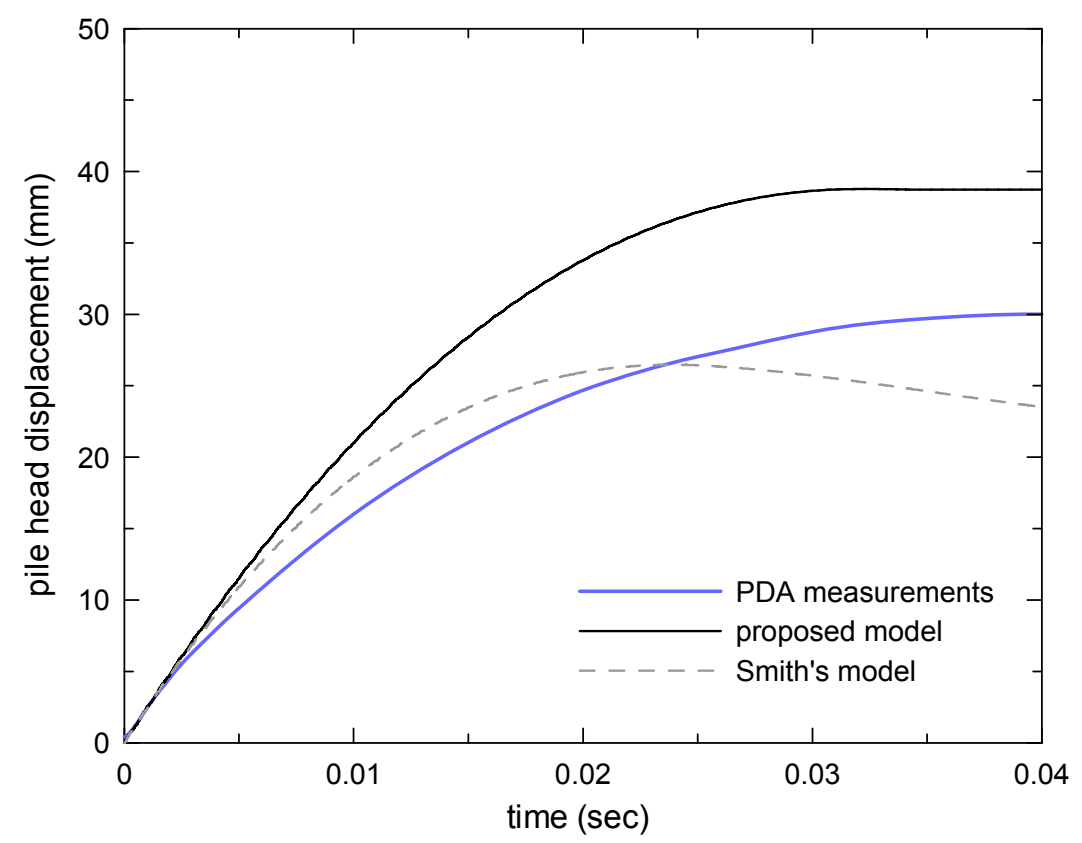

Figure 7.5. Predicted and measured pile head displacement time history for model pile test No.4: fine Ottawa sand with $D_{\mathrm{R}}=36 \%$.

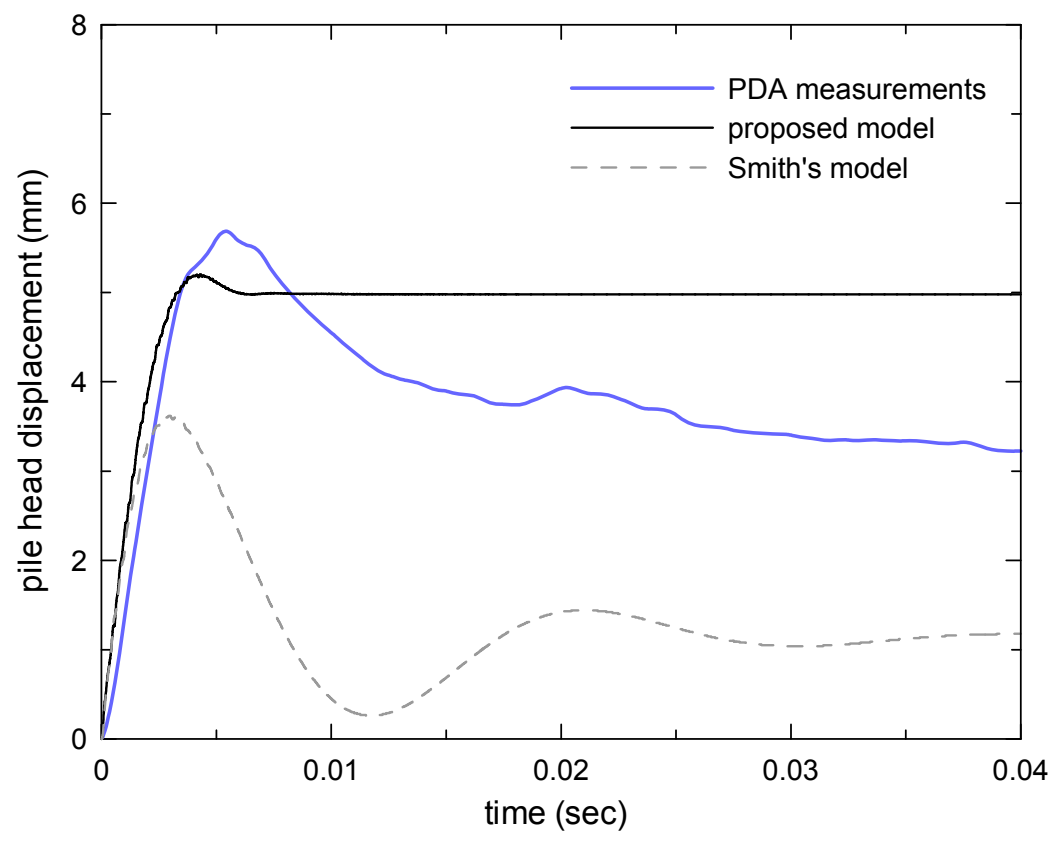

Figure 7.6. Predicted and measured pile head displacement time history for model pile test No.5: fine Ottawa sand with $D_{\mathrm{R}}=80 \%$. 


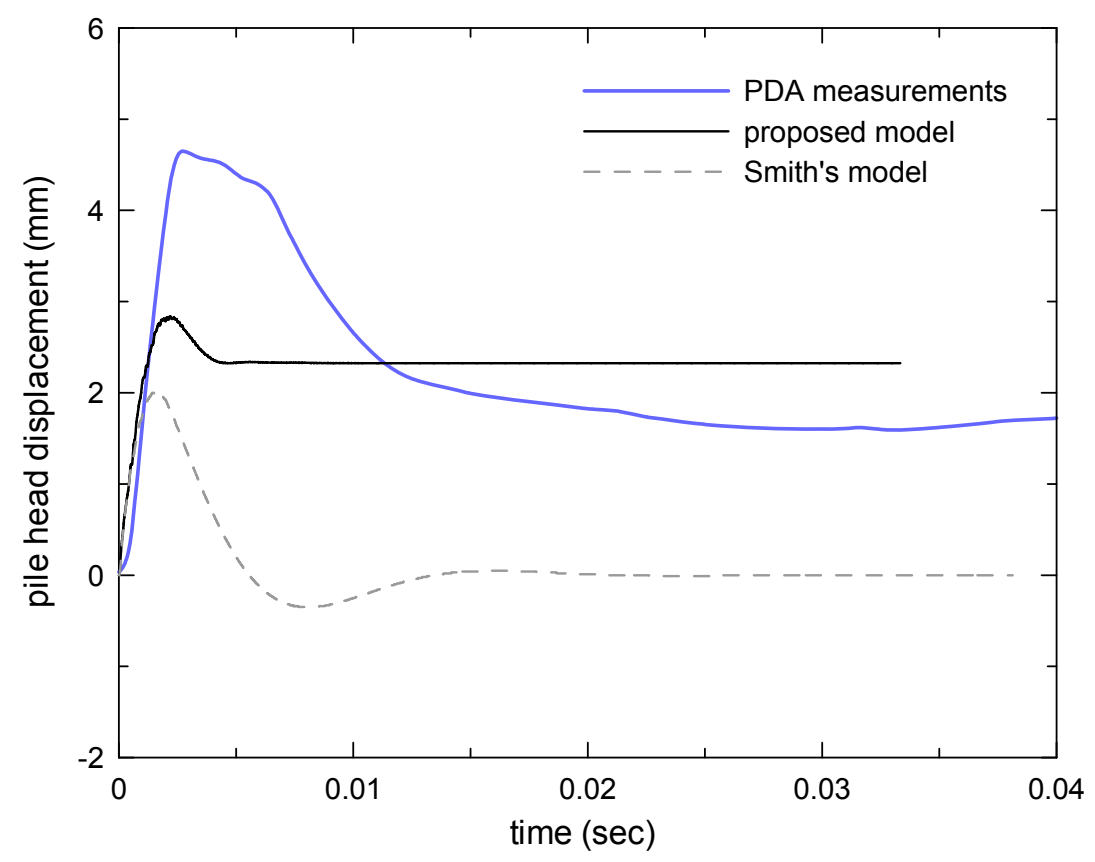

Figure 7.7. Predicted and measured pile head displacement time history for model pile test No.6: fine Ottawa sand with $D_{\mathrm{R}}=80 \%$.

With the exception of test No.5, the predicted final pile head displacement using the proposed reaction models is roughly $\pm 30 \%$ different from the one recorded by the PDA. In contrast, the analyses using the Smith (1960) analysis significantly underpredict the final set. The discrepancies become particularly severe in the case of dense sand, in which the Smith-based analysis predicts penetration refusal. The main implication of the significant pile set underprediction is that back-calculation of pile capacity using signal matching and Smith's reaction model would yield overly conservative results. The analyses with the proposed models yield displacement time history curves with similar shape as the measured ones. At the initial stage, the displacement increases until a peak values is attained. Then the displacement decreases to a residual values with minimal or no oscillations. The displacement time histories obtained from the analyses with the Smith (1960) reaction models exhibit high amplitude oscillation that have a slow rate of dissipation with time. 
Table 7.1. Experimental data and numerical predication on pile set for model piles tests.

\begin{tabular}{|c|c|c|c|c|c|c|c|c|}
\hline $\begin{array}{c}\text { Test } \\
\#\end{array}$ & Location & Pile & Sand & $\begin{array}{c}\text { Relative } \\
\text { density }\end{array}$ & $\begin{array}{c}\text { Pile } \\
\text { penetration } \\
\text { depth }(\mathrm{m})\end{array}$ & $\begin{array}{c}\text { Observed } \\
\text { pile set } \\
(\mathrm{mm})\end{array}$ & $\begin{array}{c}\text { Pile set } \\
\text { form } \\
\text { PDA } \\
(\mathrm{mm})\end{array}$ & $\begin{array}{c}\text { Predicted pile } \\
\text { set using } \\
\text { proposed model } \\
(\mathrm{mm})\end{array}$ \\
\hline 1 & test pit & I & medium & $\mathrm{D}_{\mathrm{R}}=20 \%$ & 0.86 & 27.00 & 24.00 & 19.70 \\
\hline 2 & test pit & I & medium & $\mathrm{D}_{\mathrm{R}}=95 \%$ & 0.70 & 0.70 & 0.84 & 0.86 \\
\hline 3 & test pit & II & fine & $\mathrm{D}_{\mathrm{R}}=61 \%$ & 0.96 & 10.00 & 10.00 & 7.80 \\
\hline 4 & test pit & II & fine & $\mathrm{D}_{\mathrm{R}}=36 \%$ & 0.94 & 40.00 & 30.00 & 38.70 \\
\hline 5 & test pit & II & fine & $\mathrm{D}_{\mathrm{R}}=80 \%$ & 0.89 & 5.00 & 3.20 & 4.98 \\
\hline 6 & soil tank & II & fine & $\mathrm{D}_{\mathrm{R}}=91 \%$ & 0.92 & 5.00 & 1.80 & 2.30 \\
\hline
\end{tabular}

\subsection{Field pile load test in Lagrange County}

Data recorded during driving using the PDA showed that the energy transferred to the pile head ranged from $33 \%$ to $50 \%$ of the rated (maximum potential) hammer energy. Therefore, we scale the rated hammer energy by an efficiency factor $e_{f}$ (eq. 5.64) equal to 0.41 . The pile is divided into 8 segments. The input unit shaft and base resistances were those described in Section 6.3. The maximum soil shear modulus along the pile shaft and at the pile base was estimated based on the relative density and the initial effective confining stresses using eq. (5.41). The coefficient of lateral earth pressure at rest $K_{0}$ was assumed to be equal to 0.45 . Since steel pipe piles are made of mild steel, the coefficient $n_{\mathrm{F}}$ in eq. (5.23) was set equal to 1.22. The small-strain Poisson's ratio was assumed to be equal to 0.15 . The coefficient $b_{\mathrm{fb}}$ in eqs. (5.27) and (5.38) was equal to 2.0 and the soil viscosity parameters were $m_{\mathrm{b}}=m_{\mathrm{s}}=0.3$ and $n_{\mathrm{b}}=n_{\mathrm{s}}=0.2$.

Figure 7.8 compares the predicted displacement time history at the pile head from the simulations using the proposed soil reaction models with that predicted by analysis using the Smith (1960) reaction models $\left(Q_{\mathrm{s}}=2.5 \mathrm{~mm}, Q_{\mathrm{b}}=B / 120, J_{\mathrm{s}}=0.16 \mathrm{~s} / \mathrm{m}\right.$, and $\left.J_{\mathrm{b}}=0.5 \mathrm{~s} / \mathrm{m}\right)$. The analyses using the proposed model predict final set equal to $7.5 \mathrm{~mm}$. According to the driving log, the observed pile set was $10 \mathrm{~mm}$. Again, the analysis using Smith's model significantly underpredicts the pile set. 


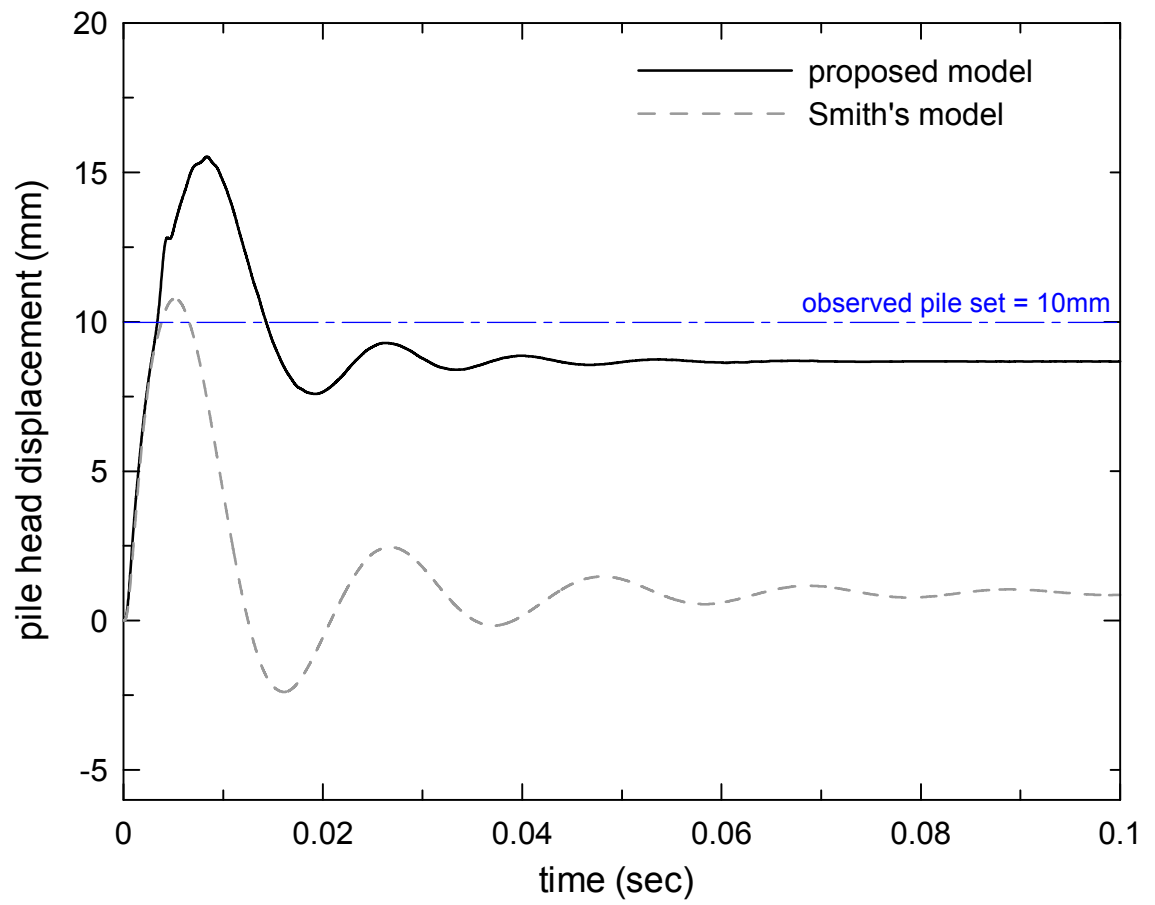

Figure 7.8. Predicted and measured pile head displacement time history for closed-ended steel pipe pile in Lagrange County. 


\section{CHAPTER 8. CONCLUSIONS AND RECOMMENDATIONS}

The main goal of the present study was to make a comprehensive assessment of the existing methods for static and dynamic analysis of pile driving, identify key shortcomings and propose improvements. Chapter 2 presented the results of interviews with two INDOT engineers and two private geotechnical consultants involved in INDOT projects. Chapter 3 compiles a collection of recommended up-to-date static design methods recently proposed in the literature. These are compared with the methods currently used in INDOT projects. Chapter 4 includes an evaluation of the existing shaft and base reaction models used in dynamic pile analyses. Chapter 5 describes the development and the formulation of the proposed improved shaft and base reaction models for 1-D dynamic pile analysis. The field and model pile tests used for the validation of the proposed reaction models are described in Chapter 6. Finally, Chapter 7 contains comparisons between field and model pile test data and the prediction from 1-D dynamic pile analysis using the proposed reaction models.

The interviews focused on the methods and procedures presently followed in deep foundation design projects. The interviews focused on the tasks and responsibilities of the parties involved in design, the type of soil input data used, the methods and software used for static design and dynamic analysis. The methods and the computer software used by private consultants involved in INDOT projects for the design of axially-loaded piles are consistent with those used by INDOT geotechnical engineers. These methods and software follow FHWA guidelines and are in accordance with standard practice in the US. Pile design is mostly based on Standard Penetration test (SPT) data and undrained shear strength measured in unconfined compression tests. Cone penetration tests are rarely performed, although the necessary equipment (CPT rigs) is available by both private soil exploration companies and INDOT. The programs DRIVEN and S-PILE are employed for calculating the static capacity of displacement piles. The commercial program SHAFT is used in the case of nondisplacement piles. Pile drivability is analyzed using the program WEAP (or GRLWEAP). Static load tests are reserved mostly for research projects. Pile driving monitoring and restrike tests are usually performed only in projects whose cost exceeds a certain limit. The computer program CAPWAP is used for back-calculating the pile capacity based on the data collected from the dynamic tests using PDA (Pile Driving Analyzer). Empirical formulas, such as ENR formula and Gates' formula, are also employed for the same purpose. 


\subsection{Static design methods}

The methods for estimating the unit shaft and base resistances currently used by geotechnical engineers (INDOT or private consultants) were developed at least twenty years ago. These methods were developed by matching predictions with a large number of (mostly noninstrumented) pile load test data without a solid theoretical basis. In the case of piles in sands, they use as input the peak friction angle, which is usually estimated based on SPT data using empirical correlations. Hence, this peak friction angle value presumably corresponds to the peak friction angle for triaxial compression conditions and for effective stresses equal to the in situ (pre-installation, geostatic) ones. In reality, the unit shaft resistance depends directly on the critical-state friction angle under simple shear conditions. In addition, the unit base resistance depends on the effective stresses existing around the pile base at limit state conditions, which are much greater than those in existence prior to installation.

Significant progress has been made in the last two decades in the field of pile foundation engineering. Numerous improved methods that are grounded on the physics and mechanics governing the development of pile resistance have been developed by combining experimental data with analysis. The new methods for the unit soil resistance in sands are substantially different from the methods currently used in practice and recommended by FHWA. The improved unit shaft resistance calculation methods use as input the critical-state friction angle instead of the peak friction angle. The new methods for the base resistance in sands use as input either the relative density or the cone resistance directly. The recently developed methods recognize the fact that the limit base resistance is almost equal to the cone penetration resistance and that the ultimate unit base resistance in sands is smaller than the limit unit base resistance. Implementing the recently developed methods in pile design practice and in design software will enhance the reliability of the design, and thus is highly recommended. A notable case is that of the unit shaft resistance of nondisplacement piles (drilled shafts) in sands. The equations for $\beta$ in the FHWA guidelines yield overly unconservative shaft resistance predictions.

CPT-based methods have many advantages and are superior to methods that rely on SPT data or other empirical methods. The CPT provides nearly direct estimate of the limit unit base resistance. This is because the mechanics involved in cone penetration are very 
similar to those involved in pile penetration. Researchers have also found that the cone resistance correlates very well with the unit shaft resistance. CPT should be performed more often in the field investigation for INDOT's deep foundation projects. Both INDOT and private companies can perform CPTs. CPT can be performed successfully in many types of soils as long as the gravel content is not excessive. If a cobble or boulder is encountered, the CPT rig will need to be relocated and penetration resumed a few feet away. Research and practice in Europe has proven that CPT can be performed without problems in dense sand down to the depths usually reached by piles.

Further research must be conducted in order to assess the accuracy and reliability of the equations for the estimation of unit shaft and base resistances. This should be done for both the equations currently used in standard practice and the more recently proposed and improved equations, for both clayey and sandy soils. Assessing the accuracy and the variability of these methods is a prerequisite for the successful development of LRFD methodologies for axially loaded piles.

\subsection{Dynamic analysis of pile driving}

One-dimensional dynamic analysis following the lumped mass approach is at this time the only practical option when it comes to pile design. Semi-analytical methods are too elaborate (and not as versatile) and the finite element method is too computationally expensive. The key components for accurate 1-D simulation of pile driving are the shaft and base soil reaction models. The computer programs used presently in practice employ the soil reaction models proposed by Smith (1960), which are characterized by a high degree of empiricism. These models are overly simple and rely on empirical constants. Researchers have developed improved soil reaction models that eliminate the empirical 'quake' parameter and attempt to more rigorously account for the different components of damping. These models are consistent with the mechanics involved in pile driving and have input parameters that are physically meaningful.

We performed a thorough review of the existing soil reaction models. Based on the shortcomings identified in the review process, we developed improved shaft and base reaction models that are consistent with the physics and mechanics of pile driving. The proposed shaft reaction model consists of a continuous annular soil disk representing the 
near-field soil surrounding the pile shaft. A plastic slider-viscous dashpot system representing the thin shear band forming at the soil-pile interface is placed at the inner boundary of the soil disk, while far-field, consistent boundaries are placed at the outer boundary of the disk. The soil in the disk is assumed to follow a hyperbolic-type stress-strain law. The base reaction model consists of a nonlinear spring and a radiation dashpot connected in parallel. The nonlinear spring is formulated in a way that reproduces realistically the base load-settlement response under static conditions. The initial spring stiffness and the radiation dashpot take into account the effect of the high base embedment. Both shaft and base reaction models capture effectively soil nonlinearity, hysteretic damping, viscous damping, and radiation damping. The input parameters of the models consist of standard geotechnical parameters, thus reducing the level of empiricism in the analysis and making it easier for engineers to make informed decisions related to input data. Data collected during the driving of full-scale piles in the field and model piles in the laboratory were used for validating the proposed models. The predictions using the proposed shaft and base reaction models compared favorably with measured pile driving data. It was shown in Chapter 7 that the simulations using the proposed models better match observations than those using the Smith (1960) models.

The pile dynamic analysis conducted in the context of this study consisted of computing the pile motion given the unit resistance values along the shaft and at the base and the rated energy of the hammer. For performing the 1-D pile driving simulations, the unit soil resistances can be estimated using the equations presented in Chapter 3 and the profile and soil property data contained in any given geotechnical report. A more important practical application would have been the back-calculation of the pile base and shaft capacities based on the force and velocity time histories measured at the pile head during driving or during a restrike test. Adding this capability to the present analysis should be part of INDOT's future research efforts. This additional capability requires the implementation of an efficient signal matching algorithm (e.g. Goble and Rausche 1979; Courage and Van Foeken, 1992; Charue 2004). The algorithm will perform a series of dynamic pile analyses using as input the measured force time history at the pile head. The values of unit soil resistance would change automatically between simulations with the ultimate goal of obtaining an adequately good match between the predicted and the measured velocity histories at the pile head, as done by the program CAPWAP. The methodology followed by CAPWAP does not require knowledge of any soil data other than the soil type. A possible alternative approach could be performing a series of simulations using as input the initial 
potential energy of the ram and trying to get a match on the observed pile set (the final pile head displacement after each hammer blow) and the peak velocity at the head (measured by the PDA). In this case, some information about the soil properties (anchor values), such as the relative density, the undrained shear strength, and the overburden effective stress, estimated from the geotechnical report data, would need to be provided as input. The algorithm would then try to match the predicted and observed pile set and peak velocity by slightly adjusting the inputted soil property values.

Finally, the creation of a public access database of pile load test results is highly recommended. Access to such data will be beneficial for private firms, INDOT engineers, and academic researchers for developing pile design knowledge that is suitable for local soils types. INDOT should also consider creating a field test site, where innovations in technology and design could be explored. 



\section{LIST OF REFERENCES}

Aoki, N. and Velloso, D.A. (1975) "An Approximate Method To Estimate The Bearing Capacity Of Piles." Proceedings, Fifth Pan-American Conference of Soil Mechanics and Foundation Engineering, Buenos Aires, Vol. 1, 367-376.

Aoki, N., Velloso, D.A. and Salamoni, J.A. (1978). "Fundações para o Silo Vertical de 100000 t no Porto de Paranaguá." 6th Brazilian Conference of Soil Mechanics and Foundation Engineering, v.3, 125-132.

Aoki, N. and de Mello V.F.B (1992). "Dynamic loading test curves." Proceedings of the Fourth International Conference on the Application of Stress-Wave Theory to Piles, The Hague, The Netherlands, 525-530.

API (1993). "Recommended Practice for Planning, Designing and Constructing Fixed Offshore Platforms." Working Stress Design, API RP2A, 20th ed. American Petroleum Institute, Washington, DC.

Basu, P., Loukidis, D., Prezzi, M. and Salgado, R. (2008). "Numerical prediction of shaft capacity for jacked piles in sands." (submitted for publication to Géotechnique).

Berezantsev, V. G. et al. (1961). "Load Bearing Capacity and Deformation of Pile Foundations." Proceedings, Fifth International Conference of Soil Mechanics and Foundation Engineering, Paris.

Beringen F.L., Hooydonk van W.R., and Schaap L.H.J. (1980). "Dynamic Pile Testing: An Aid in Analyzing Driving Behavior." Proceedings, International Seminar on the Application of Stress-Wave Theory on Piles, 77-98, Stockholm

Bolton, M. D. (1986). Strength and dilatancy of sands. Géotechnique, 36(1), 65-78.

Borja, R.I. (1988). "Dynamics of Pile Driving by the Finite Element Method." Computers and Geotechnics, 5(11), 39-49.

Bowles, J.E. (1986). Foundation analysis and design, $5^{\text {th }}$ edition, McGraw-Hill, New York.

Brown, M.J., Hyde, A.F.L. and Anderson, W.F. (2006). "Analysis of a rapid load test on an instrumented bored pile in clay." Géotechnique, 56(9), 627-638.

Briaud, J.L., and Tucker, L. (1984). "Piles in Sand: A Method Including Residual Stresses." Journal of Geotechnical Engineering, 110(11), 1666-1680.

Burland, J.B. (1970). "Discussion on Session A." Proceedings of the Conference on In situ Investigation in Soils and Rocks, British Geotechnical Society, London, England, 6162. 
Carraro, J.A.H. (2004). "Mechanical Behavior of silty and clayey sands." Ph.D. Dissertation, Purdue University.

Carraro, J.A.H., Bandini, P. and Salgado, R. (2003). "Liquefaction resistance of clean and nonplastic silty sands based on cone penetration resistance." Journal of Geotechnical and Geoenvironmental Engineering, ASCE, 129(11), 965-976.

Charue, N. (2004). "Loading rate effects on pile load-displacement behaviour derived from back-analysis of two load testing procedures." Ph.D. Dissertation, Université Catholique de Louvain, Belgium.

Chow, F.C. (1997). "Investigations in the Behavior of Displacement Piles for Offshore Foundations." Ph.D. Thesis, Imperial College, U.K.

Colombi, A. (2005). "Physical modeling of an isolated pile in coarse grained soil." Ph.D. Thesis. University of Ferrara.

Cook, R. D., Malkaus, D. S., Plesha, M. E., and Witt, R. J. (2002). Concepts and Applications of Finite Analysis. John Wiley \& Sons, New York.

Courage, W.M.G. and Van Foeken, R.J. (1992). "TNOWAVE automatic signal matching for dynamic load testing." Proceedings of the 4th international Conference on the application of the stress-wave theory to piles, The Hague, The Netherlands, 241-246.

Coutinho, A.L.G.A, Costa, A.M., Alves, J.L.D., Landau, L., and Ebecken, N.F.F. (1988) "Pile Driving Simulation and Analysis by the Finite Element Method." Proceedings, Third International Conference on the Application of Stress-Wave Theory to Piles, 197207, Vancouver, Canada.

Coyle, H.M. and Gibson, G.C. (1970). "Empirical Damping Constants for Sands and Clays." Journal, Soil Mechanics and Foundations Division, 96(SM3), 949-965.

Daya,I U. and Allen, J.H. (1975). "The effect of penetration rate on the strength of remoulded clay and sand sample." Canadian Geotechnical Journal, 12, 336-348.

De Josselin De Jong, G. (1956). "Wat gebeurt er in de grond tijdens het heien." De Ingenieur, 25, Breda, The Netherlands.

Deeks, A.J. (1992) "Numerical Analysis of Pile Driving Dynamics." Ph.D. Thesis, University of Western Australia.

Deeks, A.J. and Randolph, M.F. (1995). "A Simple Model for Inelastic Footing Response to Transient Loading." International Journal for Numerical and Analytical Methods in Geomechanics, 19(5), 307-329.

Dobry, R. and Gazetas, G. (1986). "Dynamic response of arbitrarily shaped foundations." Journal of Geotechnical Engineering, ASCE, 112(2), 109-135. 
Dolwin, J., Leonard, C.L., and Poskitt, T.J. (1979). "A study of two instrumented piles during driving." Paper No. E2006, Department of Civil Engineering, Queen Mary College, University of London.

Duncan, J.M. and Chang, C.Y. (1970). "Nonlinear analysis of stress-strain in soils." Journal of Soil Mechanics and Foundations Division, ASCE, 96(5), 1629-1653.

El-Naggar, M. and Novak, M. (1994). "Non-Linear Model for Dynamic Axial Pile Response", Journal of Geotech. Eng. Div., ASCE, 120(2), 308-329

Espinoza, D. (1991). "Application of Wave Propagation Theory in Pile Driving Analysis." Internal Report AAE-646. West Lafayette, IN: Purdue University, December

Fahey, M. and Carter, J.P. (1993). "A finite element study of the pressuremeter test in sand using a nonlinear elastic plastic model." Canadian Geotechnical Journal, 30(2), 348362.

Fioravante, V. (2002). "On the shaft friction modeling of non-displacement piles in sand." Soils and Foundations, 42(2), 23-33.

Flemming W.G.K. (1958). "The Bearing Capacity of Pile Groups.” PhD Thesis, Queen's University of Belfast.

Fleming, W.G.K., Weltman, A.J., Randolph, M.F. and Elson, W.K. (1992). Piling Engineering. Surrey University Press.

Foeken van R.J., Daniels, B., and Middendorp, P. (1996). "An Improved Method for the Real Time Calculation of Soil Resistance During Driving." Proceedings, Fifth International Conference on the Application of Stress-Wave Theory to Piles, 1132-1143, Orlando, FL.

Forehand, P.W. and Reese, J.L. (1964). "Prediction of Pile Capacity by the Wave Equation." Journal, Soil Mechanics and Foundations Division, 90(SM 2), 1-25

Fox, E.N. (1948). "The mean elastic settlement of a uniformly loaded area at adepth below the ground surface." Proceedings of the Second International Conference on Soil Mechanics and Foundation Engineering, Rotterdam, Vol. I., 129- 132.

Foye, K. C., Abou-Jaoude, G., and Salgado, R. (2004). "Limit States Design (LSD) for Shallow and Deep Foundations", Report, Joint Transportation Research Program, Report No. FHWA/IN/JTRP-2004/21.

Foye, K. C., Abou-Jaoude, G., Prezzi, M., and Salgado, R. (2006). "Resistance Factors for Use in Load and Resistance Factor Design of Driven Pipe Piles in Sands", Journal of Geotechnical and Geoenvironmental Engineering, ASCE, accepted for publication.

Gazetas, G. (1991). "Foundation vibrations." Foundation Engineering Handbook, $2^{\text {nd }}$ edition, Van Nostrand Reinholds, 553-593. 
Gazetas, G., Dobry, R. and Tassoulas, J.L. (1985). "Vertical response of arbitrarily shaped embedded foundations." Journal of Geotechnical Engineering, ASCE,111(6), 750771.

Glanville, W.H., Grime, G., Fox, E.N, and Davies, W.W (1938). "An Investigation of the Stresses in Reinforced Concrete Piles During Driving." British Building Research, Technical Paper No. 20.

Goble G.G., Likins G., and Rausche F. (1975). "Bearing capacity of piles from dynamic measurements." Final Report, Department Of Civil Engineering, Case Western Reserve University, Cleveland, Ohio.

Goble, G.G., and Rausche, F. (1976). "Wave Equation Analysis of Pile Driving, WEAP Program." Federal Highway Administration Report FHWA-IP-76-14.

Goble, G.G., and Rausche, F. (1986). "Wave Equation Analysis of Pile Foundations, WEAP86 Program." Federal Highway Administration Report Contract DTFH61-84-C00100.

Goble, G.G. and Rausche, F. (1979). "Pile drivability predictions by CAPWAP." Numeric Methods in Offshore Piling, Institution of Civil Engineers, London, 29-36.

Goble G.G., Rausche F., And Likins G. (1980). "The Analysis of Pile Driving - A State-OfThe-Art." Proceedings, International Seminar on the Application of Stress-Wave Theory on Piles, 131-161.

Hannigan P.J., Goble G.G., Thendean G., Likins G.E., and Rausche F. (1996). "Design and Construction of Driven Pile Foundations." Workshop Manual, Publication No. FHWAHI-97-014, 1996.

Hansen, B., and Denver, H. (1980). "Wave Equation Analysis of a Pile - An Analytic Model." Proceedings, International Seminar on the Application of Stress-Wave Theory on Piles, 3-22, Stockholm.

Hardin, B.O. and Black, W. L. (1968). "Vibration Modulus of Normally Consolidated Clay" Journal Soil Mechanics and Foundations Division, ASCE, 94(SM2), 353-369.

Hardin, B.O. and Drnevich, V.P. (1972). "Shear Modulus and Damping in Soils: Design Equations and Curves" Journal Soil Mechanics and Foundations Division, ASCE, 98(SM7), 667-692.

Heerema, E.P. (1979). "Relationships between wall friction, displacement, velocity and horizontal stress in clay and in sand for pile driveability analysis." Ground Engineering, 12(1), 55-61.

Heerema, E.P. (1981). "Dynamic point resistance in sand and in clay for pile drivability analysis." Ground Engineering, 14(6), 30-46.

Hejazi, H.A. (1963). "The Influence of Forced Longitudinal Vibration on Rods Penetrating Soils." Ph.D. Thesis, Ohio State University, Columbus, OH. 
Hirsch, T.J., Carr, L., and Lowery, L.L. Jr. (1976) "Pile Driving Analysis - Wave Equation User's Manual, TTI Program." Federal Highway Administration Project FWHA-IP-7613 (4 vols.)

Holeyman, A. E. (1985). "Dynamic Non-Linear Skin Friction of Piles." Proceedings of the International Symposium on Penetrability and Driveability of Piles, Vol 1, San Francisco, California, 173-176

Holeyman, A. E. (1988). "Modeling of Dynamic Behaviour at the Pile Base,", Proceedings of the $3^{\text {rd }}$ International Conference on the Application of Stress-Wave Theory to Piles, Vancouver, Canada, 174-185.

Holeyman, A., Legrand, C., and Van Rompaey, D. (1996). "A method to predict the drivability of vibratory driven piles. Proceedings of the $5^{\text {th }}$ International Conference on the Application of Stress-Wave Theory to Piles, Orlando, Florida, USA, 1101-1112.

Holloway, D. M., Audibert, J. M. E., and Dover, A. R. (1978). "Recent Advances in Predicting Pile Driveability." Proceedings, Offshore Technology Conference, OTC 3273.

Hu, Y., Randolph, M.F., and Watson, P.G. (1999). "Bearing response of skirted foundation on nonhomogeneous soil." Journal of Geotechnical and Geoenvironmental Engineering, ASCE, 125(11), 924-935.

$\mathrm{Hu}, \mathrm{Y}$ and Randolph, M.F. (2002). "Bearing Capacity of Caisson Foundations on Normally Consolidated Clay." Soils and Foundations, 42(5), 71-77.

Hussein, M., Rausche, F., and Likins, G. (1995). "Computer-Based Wave Equation Analysis of Pile Driveability." Proceedings, Second Congress on Computing in Civil Engineering, v.2, 915-926, Atlanta, Georgia, USA.

Indiana Department of Transportation, "Geotechnical Manual", Materials and Tests Division, 2005.

Ishibashi, I. and Zhang, X. (1993). "Unified Dynamic Shear Moduli and Damping Ratios of Sand and Clay." Soils and Foundations, 33(1), 182-191

Jardine R.J., Overy R.F., and Chow F.C. (1998). "Axial capacity of offshore piles in dense North Sea sands." Journal of Geotechnical and Geoenvironmental Engineering, ASCE, 124(2), 171-178.

Kim, T.C. and Novak, M. (1981). "Dynamic properties of some cohesive soils." Canadian Geotechnical Journal, 18, 371-389.

Kim, K., Salgado, R., and Lee, J. (2002). "'Load Tests on Pipe Piles for Development of CPT-Based Design Method." JTRP Project SPR-2361 Final Report.

Kondner, R.L. (1963). "Hyperbolic stress-strain response: Cohesive soil." Journal of Soil Mechanics and Foundations Division, ASCE, 189(1), 115-143. 
Kramer, S.L. (1996). “Geotechnical Earthquake Engineering”, Prentice Hall.

Lee, Jintae (2008). "Experimental Investigation of the Load Response of Model Piles Subjected to Combined Loads." Ph.D. thesis, Purdue University (in preparation).

Lee, J. and Salgado, R. (1999). "Determination of Pile Base Resistance in Sands." Journal of Geotechnical and Geoenvironmental Engineering, ASCE, Vol. 125(8), August, 673-683.

Lee, J., Salgado, R. and Paik, K. (2003). "Estimation of the Load Capacity of Pipe Piles in Sand Based on CPT Results." Journal of Geotechnical and Geoenvironmental Engineering, ASCE, 129(5), 391-403.

Lee, K.M. and Xiao, Z.R. (1999): "A New Analytical Model For Settlement Analysis Of A Single Pile In Multi-Layered Soil," Soils and Foundations, 39(5), 131-143.

Lee, S.L., Chow, Y.K., Karunaratne, G.P., and Wong, K.Y. (1988). "Rational Wave Equation Model For Pile-Driving Analysis." Journal of Geotechnical Engineering, 114(3), 306325.

Lehane B.M., Jardine R.J., Bond A.J. and Frank R. (1993). "Mechanisms of Shaft Friction in Sand from Instrumented Pile Tests." Journal of Geotechnical Engineering, ASCE, 119(1), 19-35.

Lehane, B.M., and Randolph, M. F. (2002). "Evaluation Of A Minimum Base Resistance For Driven Pipe Piles In Siliceous Sand." J. Geotech. and Geoenvir. Engrg., ASCE, 128(3), 198-205.

Lehane B.M., Schneider J.A. and Xu X. (2005). "The UWA-05 method for prediction of axial capacity of driven piles in sand." Proceedings of international symposium on frontiers in offshore geotechnics, Perth, Australia, 683-689.

Liang, R.Y. (2000). "Correlative study of Smith damping coefficient and SPT blow count." Proceedings of the 6th International Conference on the Application of Stress-Wave Theory to Piles, São Paulo, Brazil, 461-467.

Liang, R.Y. and Sheng, Y. (1992). "Theoretical Interpretation of Smith' Model Parameters." Proceedings, Fourth International Conference on the Application of Stress-Wave Theory to Piles, The Hague, The Netherlands, 111-116.

Likins, G., Rausche, F., DiMaggio, J., and Teferra, W., (1992). "A solution for high damping constants in sands." Proceedings, Fourth International Conference on Application of Stress-Wave Theory to Piles, The Hague, The Netherlands, 117-120.

Litkouhi, S. and Poskitt, T.J. (1980). "Damping Constants for Driveability Calculations." Geotechnique, 30(1), 77-86. 
Lings, M.L. and Dietz, M.S. (2005). "The peak strength of sand-steel interfaces and the role of dilation." Soils and Foundations, 45(6), 1-14.

Liyanapathirana, D.S., Deeks, A.J. and Randolph, M.F. (2000). "Numerical Modelling Of Large Deformations Associated With Driving Of Open-Ended Piles", International Journal for Numerical and Analytical Methods in Geomechanics, 24, 1079-1101

Lopes, F.R. and H. Laprovitera (1988). "On the Prediction of the Bearing Capacity of Bored Piles from Dynamic Penetration Tests." In: Van Impe(ed), Deep Foundations on Bored and Auger Piles: 537-540. Rotterdam: Balkema.

Loukidis D. (2006). "Advanced constitutive modeling of sands and applications to foundation engineering." Ph.D. thesis, Purdue University.

Loukidis, D. and Salgado, R. (2008). "Analysis of the shaft resistance of non-displacement piles in sand." Géotechnique, 58(4), 283-296.

Lowery, L.L. Jr., Hirsch, T.J., Edwards, T.C., Coyle, H.M. and Samson, C.H. Jr. (1969). "Pile Driving Analysis - State of the Art." Research Report No. 33-13. Texas A\&M University, College Station, Texas.

Lysmer, J., and Richart, F.E. (1966). "Dynamic Response Of Footing To Vertical Loading.", Journal Eng. Mech. Div., ASCE, 92(1), 65-91.

Mabsout, M. and Tassoulas, J. (1994). "A Finite-Element Model For The Simulation Of Pile Driving", International Journal for Numerical Methods in Engineering, 37(2), 257-278

Mabsout, M., Reese, L., and Tassoulas, J. (1995). "A Study Of Pile Driving By The FiniteElement Method", Journal of Geotechnical Engineering, ASCE, 121(7), 535-543

Maksimovic, M. (1989). "On the residual shearing strength of clays." Géotechnique, 39(2), 347-351.

Martin, C.M. (2001). "Vertical Bearing Capacity of Skirted Circular Foundations on Tresca Soil." Proceedings 15th International Conference on Soil Mechanics and Geotechnical Engineering, Istanbul, Vol. 1, 743-746.

Masouleh, S. F. and Fakharian, K. (2007). "Application of a continuum numerical model for pile driving analysis and comparison with a real case." Computers and Geotechnics, 35(3), 406-418.

Mayne, P.W. and Rix, G.J. (1993). " $\mathrm{G}_{\max }$ - $\mathrm{q}_{\mathrm{c}}$ relationships for clays." Geotechnical Testing Journal, 16(1), 54-60.

McVay, M.C., Birgisson, B., Zhang, L., Perez, A. and Putcha, S. (2000). "Load and resistance factor design (LRFD) for driven piles using dynamic methods - A Florida perspective." Geotechnical Testing Journal, 23(1), 55-66.

Meyerhof, G.G. (1976). "Bearing Capacity and Settlement of Pile Foundations," Journal of Geotechnical Engineering Division, ASCE, 102(GT3), pp. 195-228. 
Meyerhof, G.G. (1983). "Scale Effects of Ultimate Pile Capacity." Journal of Geotechnical Engineering, 109(6), 797-806.

Michaelides, OD, Gazetas, G., Bouckovalas, G., and Chrysikou, E., (1997). "Approximate Non-Linear Dynamic Axial Response of Piles." Géotechnique, 48(1), 33-53.

Michaelides, OD., Bouckovalas, G., and Gazetas, G., (1998). "Non-Linear Soil Properties and Impedances for Axially Vibrating Pile Elements." Soils and Foundations, 38(3), 129-142.

Middendorp, P. and van Weele, A.F. (1986). "Application of Characteristic stress Wave Method in Offshore Practice", Proceedings, Third International Conference on Numerical Methods in Offshore Piling, Nantes, Supplement, 6-18

Mitwally, H. and Novak, M. (1988). "Pile Driving Analysis Using Shaft Models and FEM", Proceedings, Third International Conference on the Application of Stress-Wave Theory to Piles, 455-466, Vancouver, Canada

Morgano, C.M. and Liand R. (1992). "Energy transfer in SPT -Rod length effects." Proceedings of the $4^{\text {rd }}$ International Conference on the Application of Stress-Wave Theory to Piles, The Hague, The Netherlands, 121-127.

Murthy, T.G., Loukidis, D., Carraro, J.A.H., Prezzi, M. and Salgado R. (2007). "Undrained monotonic response of clean and non-plastic silty sands." Géotechnique, 57(3), 273288.

Nath, B. (1990). "A Continuum Method Of Pile Driving Analysis: Comparison With The Wave Equation Method." Computers and Geotechnics, 10(4), 265-285.

Nordlund, R.L. (1979)."Point Bearing and Shaft Friction of Piles in Sand." Proceedings, 5th Annual Fundamentals of Deep Foundation Design, University of Missouri-Rolla.

Novak, M., Nogami, T., and Aboul-Ella, F. (1978), "Dynamic Soil Reactions for Plane Strain Case", Journal of Engineering Mechanics Division, ASCE, 104(4), pp. 953-959

Nguyen, T. T., Berggren, B., and Hansbo, S., (1988). "A New Soil Model for Pile Driving and Driveability Analysis." Proceedings, Third International Conference on Application of Stress-Wave Theory to Piles, 353-367, Vancouver, Canada.

O' Neill, M.W. and Hassan, K.M. (1994). "Drilled Shafts: Effects of construction on performance and design criteria." Proceedings of the International Conference on Design and Construction of Deep Foundations, Orlando, Florida. 137-187.

O'Neill, M.W. and Resse, L.C. (1999). "Drilled shafts: Construction procedures and design methods." Report No. FHWA-IF-99-025, Federal Highway Administration, Washington, D.C.

Oda, M., Takemura, T. and Takahashi, M. (2004). "Microstructure in shear band observed by microfocus X-ray computed tomography." Géotechnique, 54(8), 539-542. 
Paik, K., Salgado, R. Lee, J and Kim, B. (2003). "The Behavior of Open- and Closed ended Piles Driven into Sands." Journal of Geotechnical and Geoenvironmental Engineering, ASCE, 129(4), 296-306.

Paikowsky S.G. and Chernauskas L.R. (1992). "Energy Approach for Capacity Evaluation of Driven Piles." Proceedings, Fourth International Conference on the Application of Stress-Wave Theory to Piles, The Hague, The Netherlands, 595-601.

Paikowsky S.G., Regan J.E., and McDonnell J.J. (1994). "A Simplified Field Method for Capacity Evaluation of Driven Piles." Publication No. FHWA-RD-94-042, 1994.

Parker, E., Guaita, P. and Rentocchini, R. (1996). "Closed Form Solution to Wave Equation for Steam and Hydraulic Hammers." Presented at the Fifth International Conference on the Application of Stress-Wave Theory to Piles, Orlando, FL.

Parkin, A. K., and Lunne, T. (1982). "Boundary Effect in the Laboratory Calibration of a Cone Penetrometer for Sand." Proceedings., Second European Symp. on Penetration Testing, Amsterdam, The Netherlands, 761-768.

Parola, J.F. (1970). "Mechanics of Impact Pile Driving." Ph.D. Thesis, University Of Illinois at Urbana-Champaign.

Peck, R.B., Hanson, W.E. and Thornburn, T.H. (1974) Foundation Engineering, John Wiley \& Sons, New York, 2nd edition.

Pells, P.J.N. and Turner, R.M. (1978). Discussion on "Janbu, Bjerrum and Kjaernsli's chart reinterpreted" by J. T. Christian and W. D. Carrier. Canadian Geotechnical Journal, $15,436-437$.

Potts D.M. and Martins J.P. (1982). "The shaft resistance of axially loaded piles in clay." Géotechnique, 32(4), 369-386.

Price, G. and I.F. Wardle (1982). A Comparison Between Cone Penetration Test Results and the Performance of Small Diameter Instrumented Piles in Stiff Clay. Proceedings, Second European Symposium on Penetration Testing, 2: 775-780. Amsterdam.

Randolph, M.F. (2003). "Science and Empiricism in Pile Foundation Design." 43rd Rankine Lecture, Geotechnique, 54(1).

Randolph, M.F. and Murphy, B.S. (1985). "Shaft Capacity of Driven Piles in Clay." OTC 4883, 371-378.

Randolph, M.F. and Simons, H.A. (1986). "An improved soil model for one-dimensional pile driving analysis." Proceedings of the 3rd International Conference of Numerical Methods in Offshore Piling, Nantes France, 3-17.

Randolph, M.F. and Wroth, C.P. (1978). "Analysis of deformation of vertically loaded piles." Journal of Geotechnical Engineering Division, ASCE, 104(GT12),1-17. 
Rausche, F. (1970). "Soil Response from Dynamic Analysis and Measurements on Piles." Thesis presented to the Case Western Reserve University, at Cleveland, Ohio, in 1970, in partial fulfillment of the requirements for the degree of Doctor of Philosophy.

Rausche, F., Goble, G.G., and Likins, G. (1985). "Dynamic Determination of Pile Capacity." Journal of Geotechnical Engineering, 111(3), 367-383.

Rausche, F., Likins, G., and Goble, G.G. (1994). "A Rational and Usable Wave Equation Soil Model Based on Field Test Correlations." Proceedings, FHWA International Conference on Design and Construction of Deep Foundations, Orlando, Florida, USA.

Reese, L.C., Touma, F.J. and O'Neill, M.W. (1976). "Behavior of Drilled Piers under Axial Loading." Journal of the Geotechnical Engineering Division, ASCE, 102(5), 493-510.

Reese, L.C., and O'Neill M.W. (1989). "New Design Method for Drilled Shafts from Common Soil and Rock Tests." Proc. of Congress Foundation Engineering: Current Principles and Practices, ASCE, Vol. 2, 1026-1039.

Rix, G.J. and Stokoe, K.H. (1991). "Correlation of initial tangent modulus and cone penetration resistance." Calibration Chamber Testing, Elsevier Science, New York, 351-361.

Robertson, P.K., Campanella, R.G., Wightman, A. (1983). "SPT-CPT correlations." Journal of Geotechnical Engineering, 109(11), 1449-1459.

Salgado, R. (1995). "Design of Piles in Sands Based on CPT Results" 10th Pan-American Conference on Soil Mechanics and Foundation Engineering, Vol. 3, 1261-1274, Guadalajara.

Salgado, R., J. K. Mitchell, and M. Jamiolkowski (1997). "Cavity Expansion and Penetration Resistance in Sand." Journal of Geotechnical and Geoenvironmental Engineering, ASCE, 123(4), 344-354

Salgado, R., Michell, J. K., and Jamiolkowski, M. (1998). "Calibration Chamber Size Effects on Penetration Resistance in Sand." Journal of Geotechnical and Geoenvironmental Engineering, 124(9), 878-888.

Salgado, R., Lee, J.H., and Kim, K. (2002). "Load Tests on Pipe Piles for Development of CPT-Based Design Method", Report, Joint Transportation Research Program, Report No. FHWA/IN/JTRP-2002/4.

Salgado, R., Lyamin, A., Sloan, S. and Yu, H.S. (2004). "Two- and Three-dimensional Bearing Capacity of Footings in Clay." Géotechnique, 54(5), 297-306.

Salgado, R. (2006a). "The role of analysis in pile design." In Modern Trends in Geomechanics." Springer Proc. in Physics, Vol. 106 (Wu, Wei; Yu, H.-S., Eds.) 
Salgado, R. (2006b). "Analysis of the Axial Response of Non-Displacement Piles in Sand." Geomechanics II: Testing, Modeling and Simulation. Proceedings, Second JapanU.S. Workshop, Geotechnical Special Publication No. 156, ASCE, 427-439.

Salgado, R. and Prezzi, M. (2007). "Computation of cavity expansion pressure and cone resistance in sand." International Journal of Geomechanics, ASCE, 7(4), 251-265.

Salgado, R. (2008). The Engineering of Foundations, McGraw-Hill.

Seed, H.B. and Idriss, I.M. (1970). "Soil Moduli and Damping Factors for Dynamic Response Analyses." Report EERC 70-10, Earthquake Engineering Center, University of California, Berkeley.

Simons, H.A. and Randolph, M.F. (1985). "A New Approach To One-Dimensional Pile Driving Analysis." Proceedings, Fifth International Conference on Numerical. Methods in Geomechanics, Nagoya, 3, 1457-1464.

Smith, E.A.L. (1960). "Pile-Driving Analysis by the Wave Equation." Journal, Soil Mechanics and Foundations Division, 86(EM 4), 35-61.

Stas, C.V. and Kulhawy, F.H. (1984). "Critical evaluation of design methods for foundations under axial uplift and compression loading." EPRI Report EL-3771, Research Project 1493-1, Electric Power Research Institute, Palo Alto, California.

Tatsuoka, F., Goto, S., Tanaka, T., Tani, K., \& Kimura, Y. (1997). "Particle size effects on bearing capacity of footing on granular material." Deformation and Progressive Failure in Geomechanics, (eds. A. Asaoka, T. Adachi \& F. Oka), Pergamon, 133138.

Thurman, A.G. (1964). "Computed Load Capacity and Movement of Friction and EndBearingPiles Embedded in Uniform and Stratified Soil." Ph.D. Thesis, Carnegie Institute of Technology.

Tomlinson, M.J. (1980). "Foundation Design and Construction." Pitman Advanced Publishing, Boston, MA, 4th edition.

Tomlinson, M.J. (1987). Pile Design and Construction Practice. Viewpoint, London.

Uesugi, M., Kishida, H. and Tsubakihira, Y. (1988). "Behavior of sand particles in sand-steel friction." Soils and Foundations, 28(1), 107-118.

Uesugi, M., Kishida, H. and Uchikawa, Y. (1990). "Friction between dry sand and concrete under monotonic and repeated loading." Soils and Foundations, 30(1), 115-128.

Uto, K., Fuyuki, M. and Sakurai, M. (1985). "An Equation for the Dynamic Bearing Capacity of a Pile Based on Wave Theory." Proceedings, International Symposium on Penetrability and Drivability of Piles, San Francisco.

Vardoulakis, I. (2002). "Dynamic thermo-poro-mechanical analysis of catastrophic landslides." Géotechnique, 52(3), 157-171. 
Van Koten, H., Middendorp, P., and Van Brederode, P. (1980). "An Analysis of Dissapative Wave Propagation in a Pile." Proceedings, International Seminar on the Application of Stress-Wave Theory On Piles, 23-40, Stockholm.

Vanden Berghe, J-F (2001). Sand strength degradation within the framework of pile vibratory driving." Doctoral Thesis, Université Catholique de Louvain, Belgium.

Vucetic, M. and Dobry, R. (1991). "Effect of Soil Plasticity on Cyclic Response". Journal of Geotechnical Engineering, ASCE, 117(1), 89-107

Wang, Y.X. (1988). "Determination of Capacity of Shaft Bearing Piles Using the Wave Equation." Proceedings, Third International Conference on the Application of StressWave Theory to Piles, 337-342. Vancouver, Canada.

Warrington, D.C. (1987). "A Proposal for a Simplified Model for the Determination of Dynamic Loads and Stresses During Pile Driving." Proceedings, Nineteenth Annual Offshore Technology Conference, Dallas, TX. OTC 5395.

Warrington, D.C. (1997). "Closed Form Solution of the Wave Equation for Piles." Master's Thesis, University of Tennessee at Chattanooga.

Weiler, W. A. (1988). "Small Strain Shear Modulus of Clay", Proceedings, ASCE Conference on Earthquake Engineering and Soil Dynamics II: Recent Advances in Ground Motion Evaluation, Geotechnical Special Publication 20, ASCE, New York, 331-335

Yu, H.S., Herrmann, L.R. and Boulanger, R.W. (2000). "Analysis of steady cone penetration in clay." Journal of Geotechnical and Geoenvironmental Engineering, ASCE, 126(7), 594-605.

Zhou, J. and Liang, R.Y. (1996). "Identification of Soil-Pile Interaction Model Parameters from HST Data." Presented at the Fifth International Conference on the Application of Stress- Wave Theory to Piles, Orlando, FL. 


\section{APPENDIX. FIELD AND MODEL PILE LOAD TEST PICTURES}

\section{A.1 Field pile load test in Jasper County}

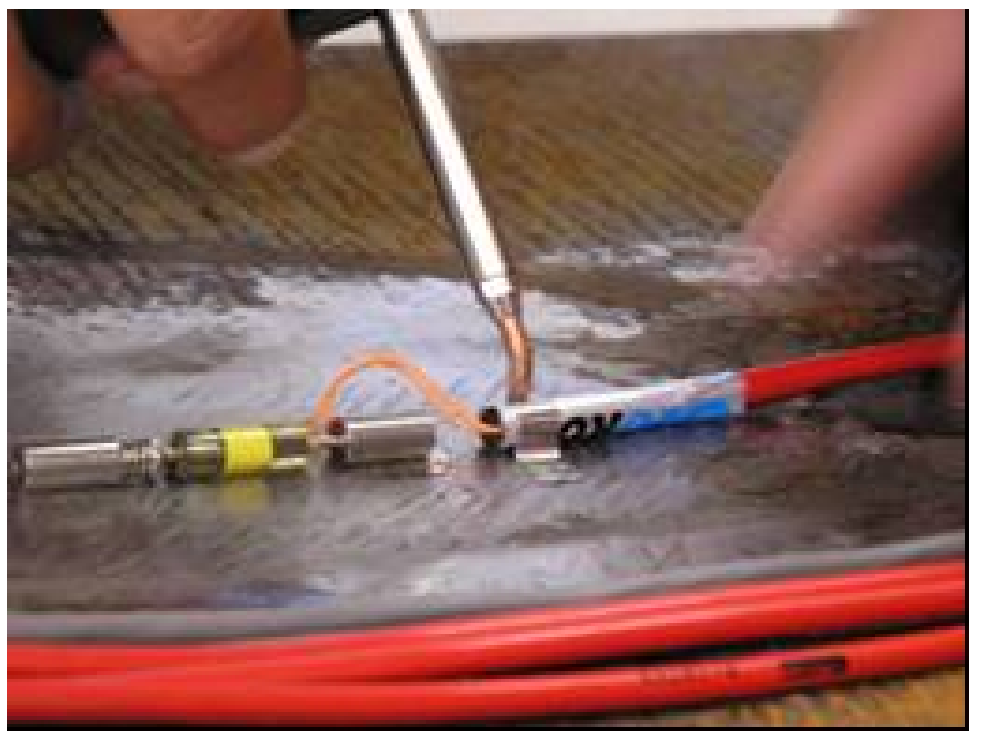

Figure A. 1 Welding of a vibrating wire strain gage to the pile shaft

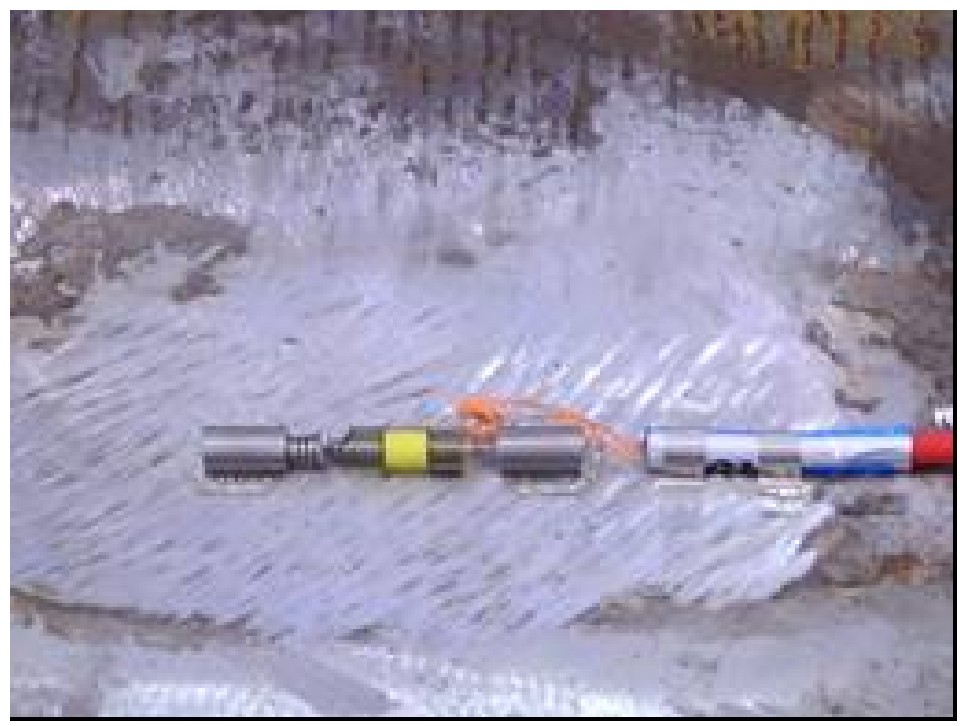

Figure A.2 Spot-welded gage on smoothed pile surface 


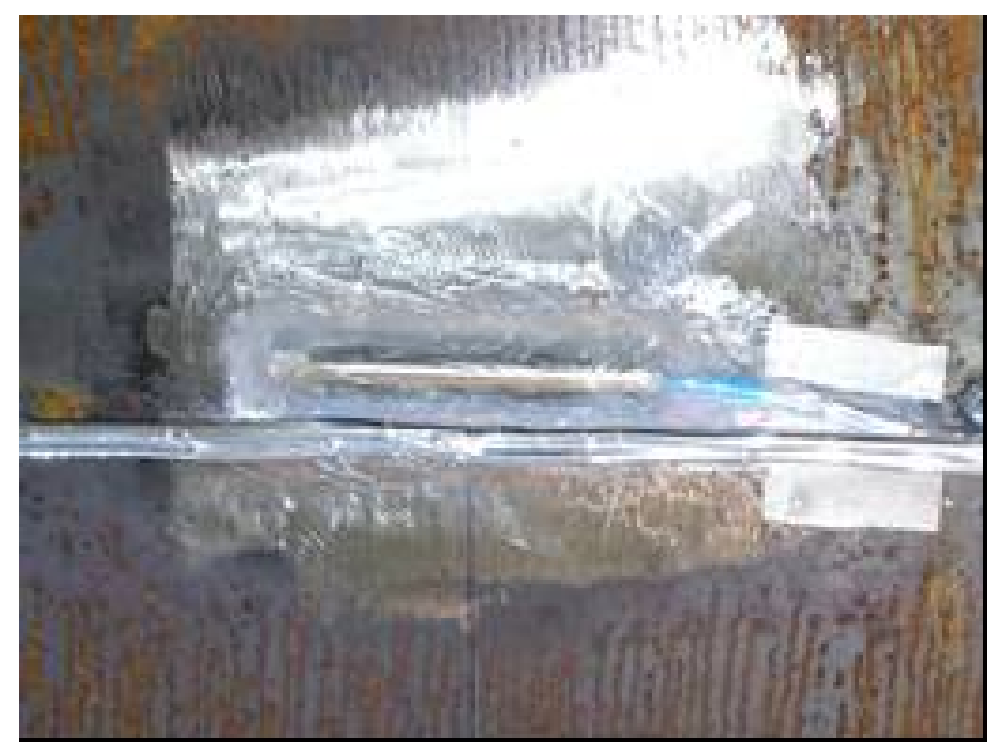

Figure A.3 Gage protected with steel cover and silicon caulking

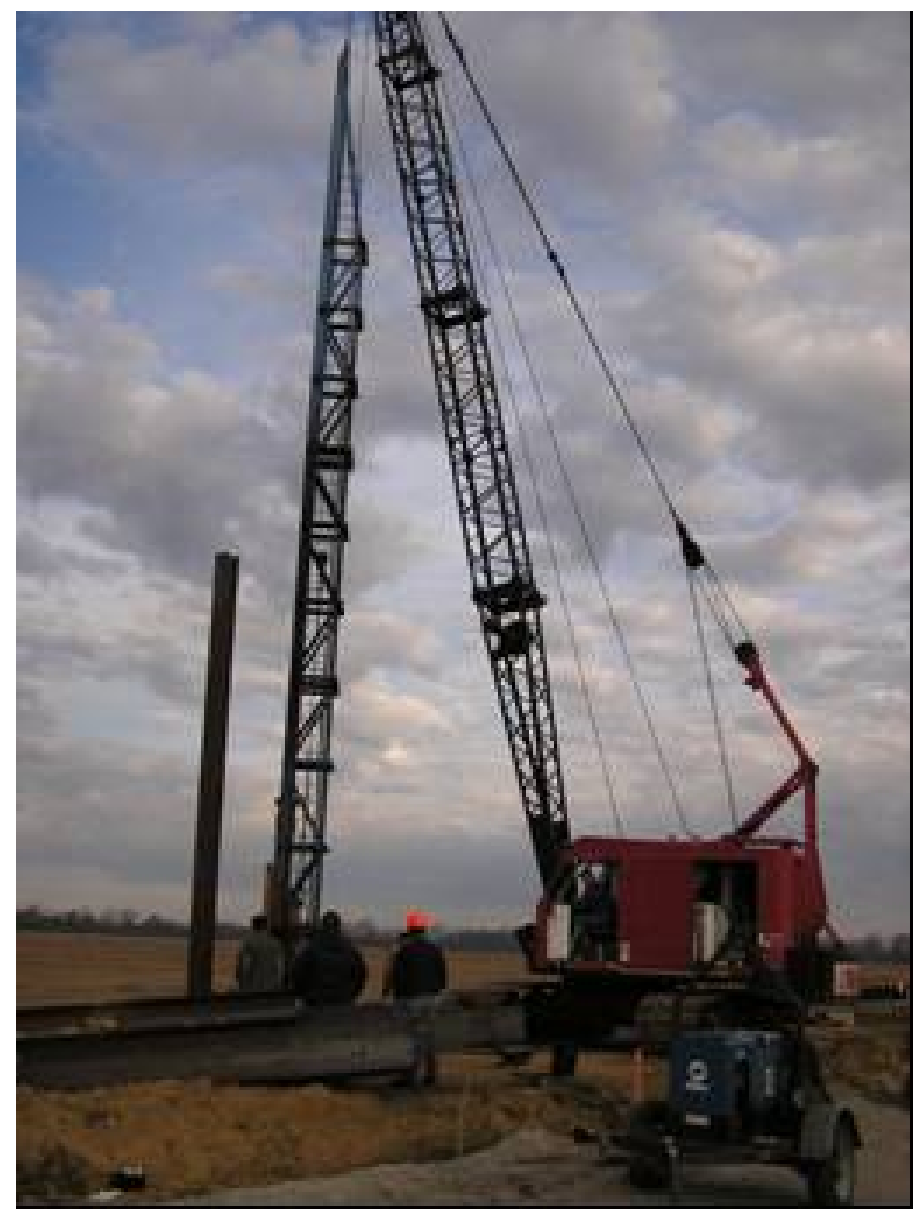

Figure A.4 ICE-42S diesel hammer used in pile driving 


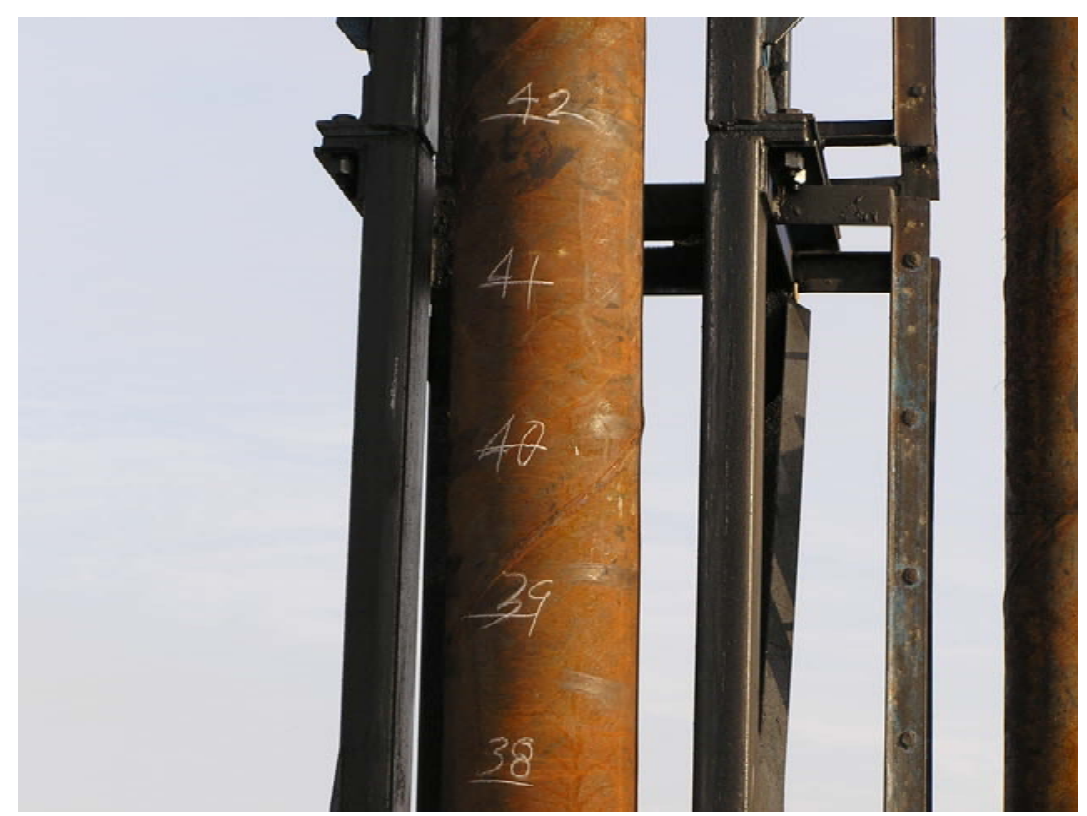

Figure A.5 Pile marked every $1 \mathrm{ft}$ for blow count records

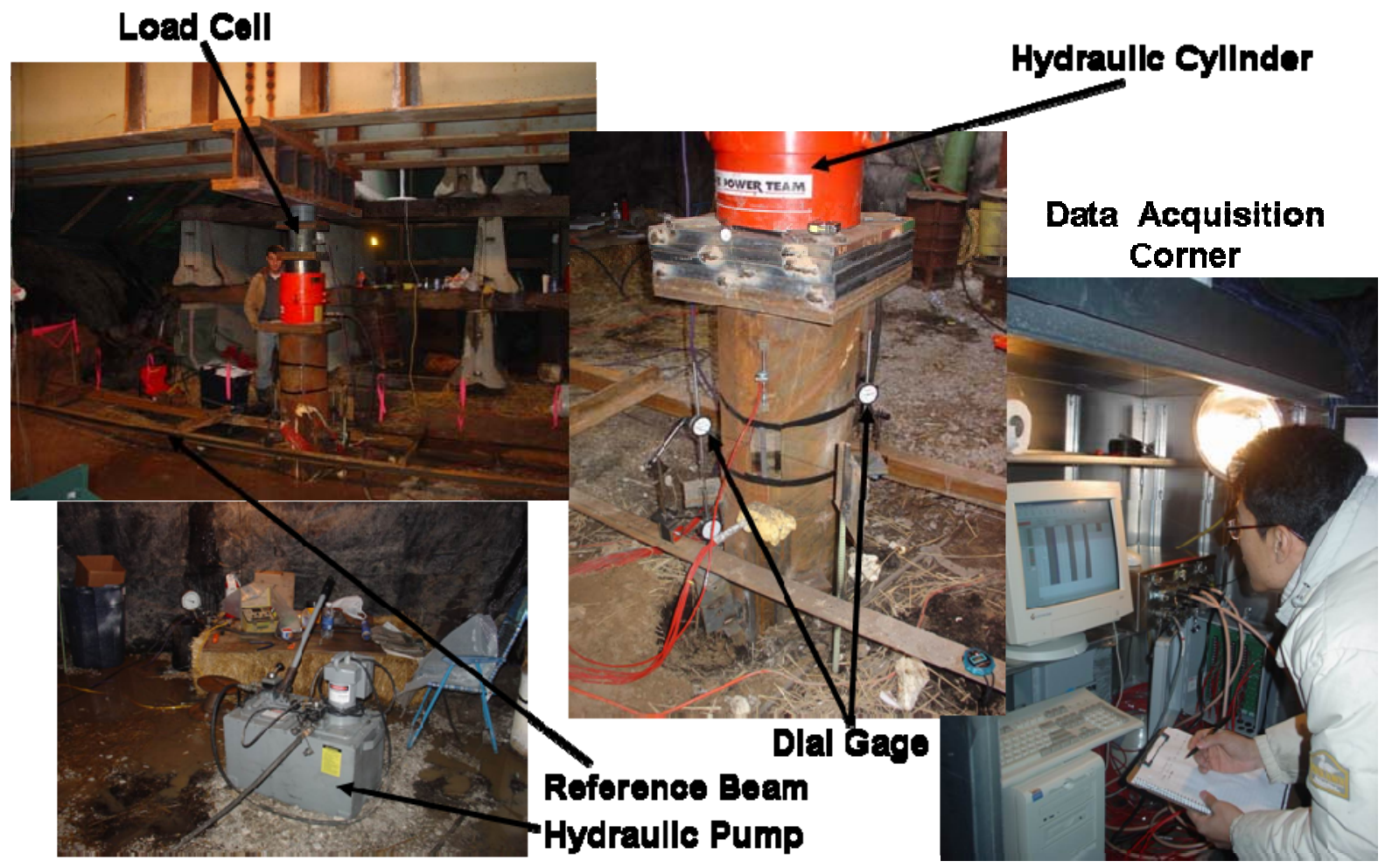

Figure A.6 Static pile load test setup 


\section{A.2 Model pile load tests in test pit}
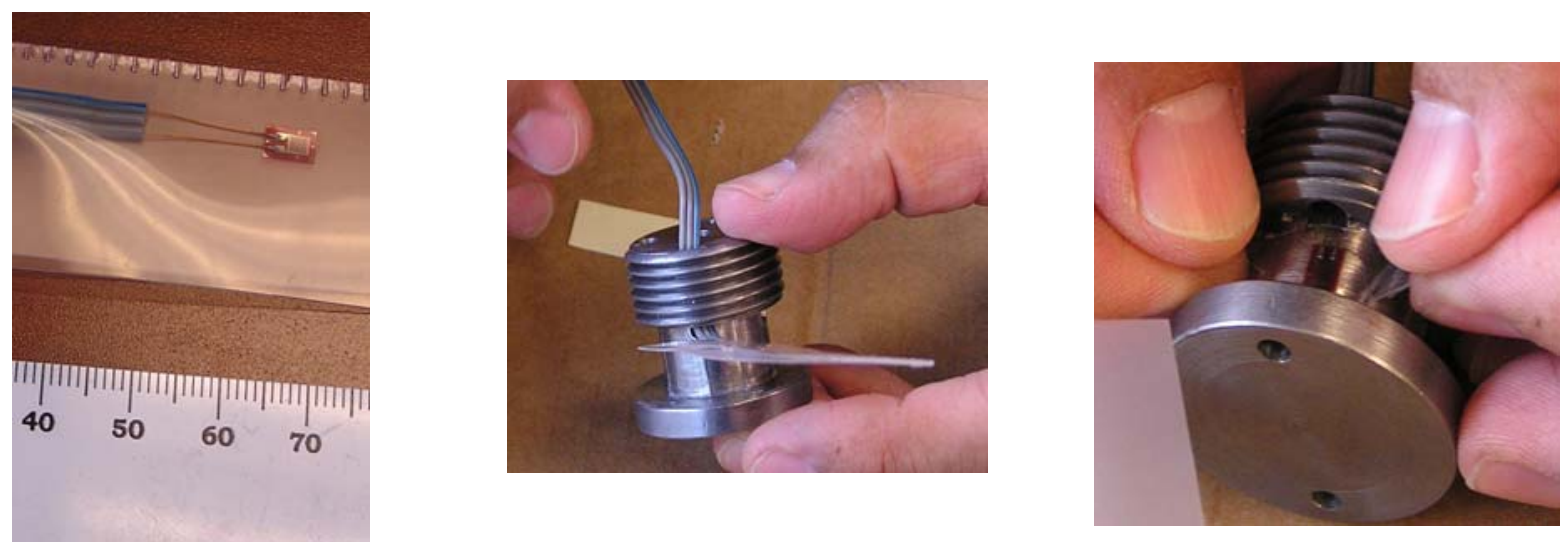

Figure A.7 Electric resistance strain gage attached to the pile base load cell
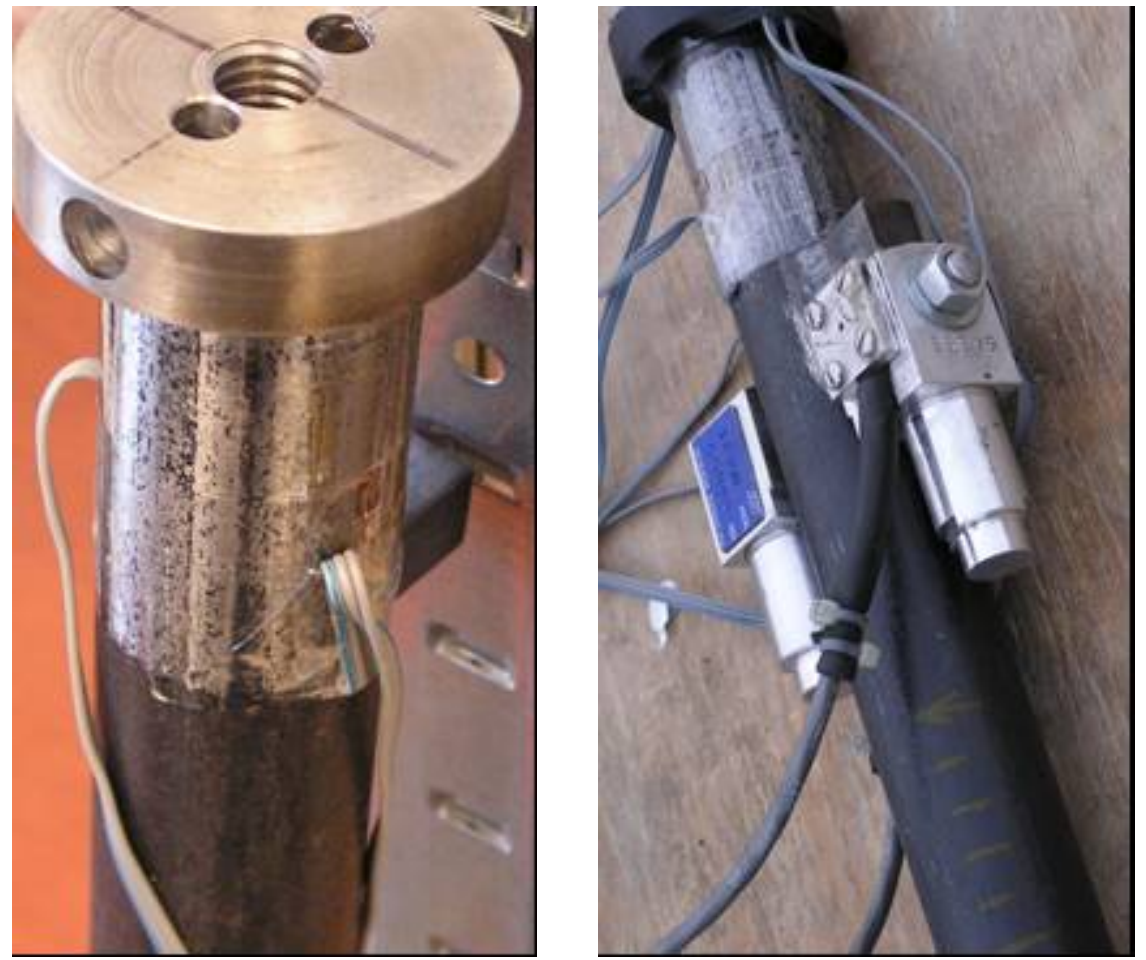

Figure A.8 Strain gages and accelerometers attached to the pile top 


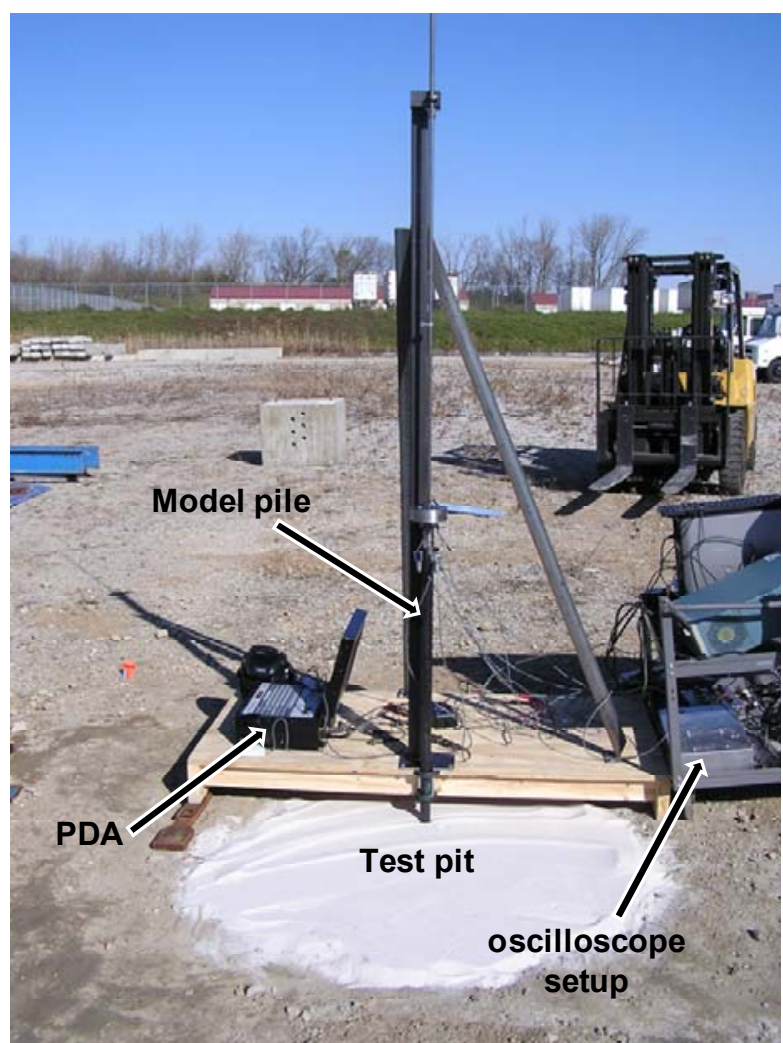

Figure A.9 Test pit and model pile setup

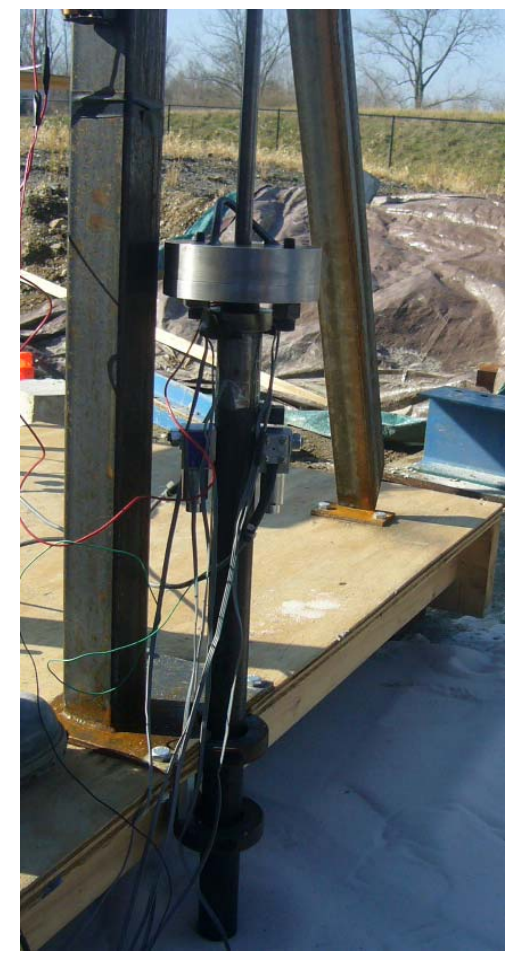

Figure A.10 Close-up on drop hammer and model pile top 


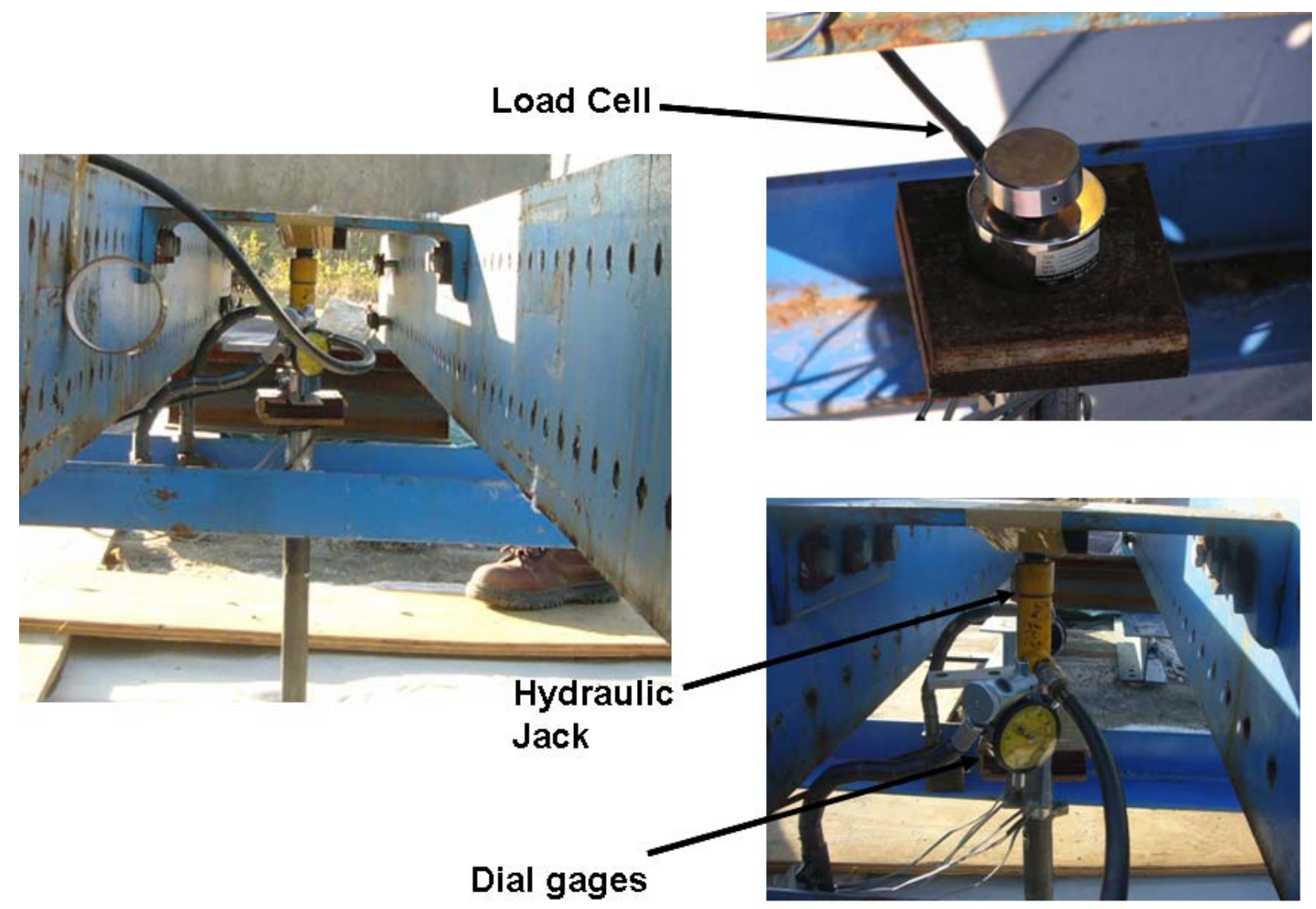

Figure A.11 Load cell and hydraulic jack setup for model pile

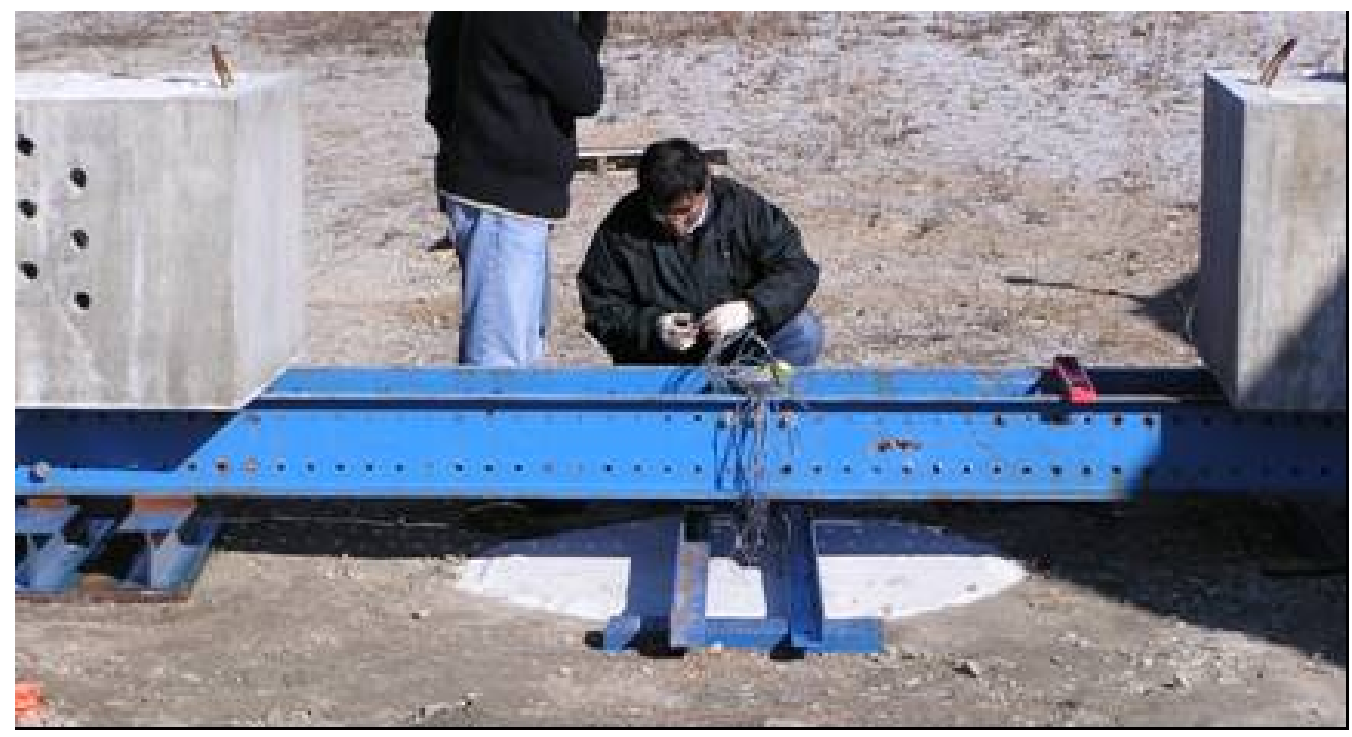

Figure A.12 Reaction beam and counter weights for model pile test 